\title{
The paracrine effects of fibroblasts on Doxorubicin-treated breast cancer cells
}

\author{
Carla Fourie
}

Dissertation presented in partial fulfilment of the requirements for the degree of Master of Science (Physiological Sciences) at

Stellenbosch University

Supervisor: Prof Anna-Mart Engelbrecht

Co-supervisor: Dr Tanja Davis

April 2019 


\section{Declaration of Originality}

\section{"Declaration}

By submitting the thesis/dissertation electronically, I declare that the entirety of the work contained therein is my own, original work, that I am the sole author thereof (save to the extent explicitly otherwise stated), that production and publication thereof by Stellenbosch University will not infringe any third party rights and that I have not previously in its entirety or in part submitted it for obtaining any qualification".

\section{Date}




\section{Abstract}

Introduction: Breast cancer is frequently diagnosed in women in both developed and developing countries and poses a major health problem throughout the world. The current standard treatment for breast cancer patients is radiation, surgery and chemotherapy or a combination of surgery with chemotherapy. The unresponsiveness of cancer cells to chemotherapeutics, however, is still a main concern. During chemotherapeutic treatment with Doxorubicin, normal and healthy neighbouring cells are also damaged. Apoptotic or senescent fibroblasts in the tumour microenvironment can then secrete a variety of bioactive molecules which promote tumour growth, metastasis and drug resistance.

Methods: Mouse embryonic fibroblasts (MEFs) were cultured and treated with Doxorubicin to induce apoptosis and senescence respectively. An SA-B-gal stain was used to determine the number of senescent cells in the cell population and expression of apoptotic and senescent markers were determined through western blotting. Conditioned media was collected from the MEFs after apoptosis and senescence induction and used to assess the paracrine effects between fibroblasts and E0771 cells.

Results and discussion: Doxorubicin $(1 \mu \mathrm{M})$ was able to significantly induce apoptosis in MEFs after 24 hours. During senescence induction, $2 \mu \mathrm{M}$ of Doxorubicin treatment for 4 hours was unable to induce $80 \%$ of senescence in the MEF population. The western blot analyses show that the expression of many apoptosis and senescence markers significantly increased or decreased after Doxorubicin treatment. Furthermore, the results indicate that senescent fibroblasts $(56 \%)$ were able to significantly increase cell viability in E0771 cells following treatment with Doxorubicin.

Conclusion: Our results highlight the fact that the tumour microenvironment is extremely complex and how important it is that chemotherapeutic agents such as Doxorubicin should specifically target cancer cells. Once healthy, neighbouring stromal cells such as fibroblasts are affected by chemotherapeutic agents, they have the ability to secrete paracrine factors that enhance breast cancer growth and induce therapeutic resistance by evading cell death. 


\section{Acknowledgements}

I would like to thank the following people:

My supervisor, Prof. A-M Engelbrecht for providing me with this opportunity and guidance throughout the year.

CRG for all the advice and support.

My parents and sister, for all the encouragement, motivation and support throughout the years.

The staff of the Physiology department for providing me with this opportunity.

National Research Foundation (NRF) for funding

Central Analytical Facilities (CAF), including Lize Engelbrecht, Rozanne Adams and Dumisile Lumkwana for technical assistance.

Jurgen Kriel for helping us with autophagy quantification.

Lastly, I would like to especially thank my co-supervisor, Tanja Davis, for all her guidance, support, help and motivation in and out of the lab throughout the year. 


\section{Table of Contents}

Declaration of Originality. i

Abstract. ii

Acknowledgements. .. iii

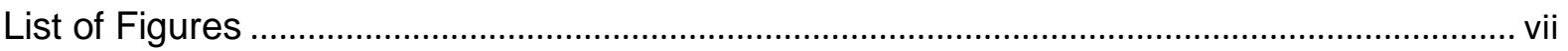

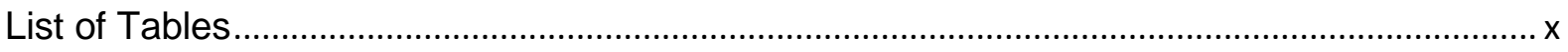

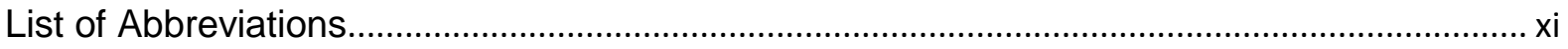

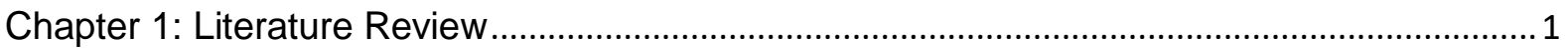

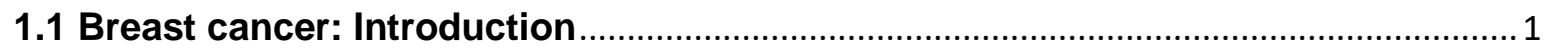

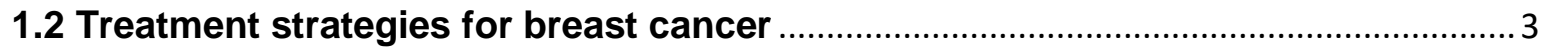

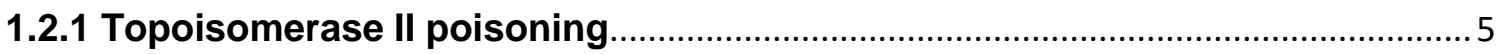

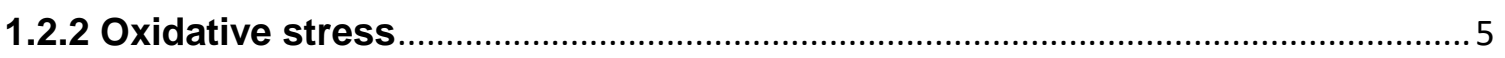

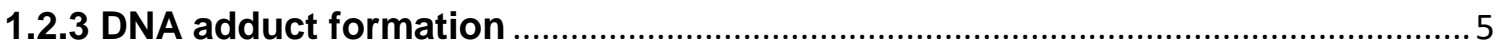

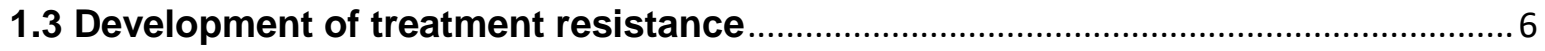

1.4 The Phosphoinositide 3-kinase pathway in cancer ................................................. 7

1.4.1 Ras/Raf/MEK/ERK signalling contributes to pro-survival signalling of cancer

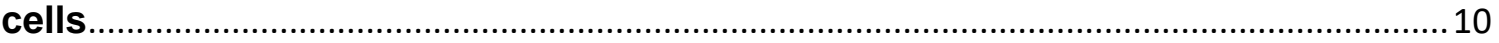

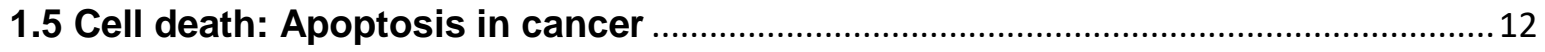

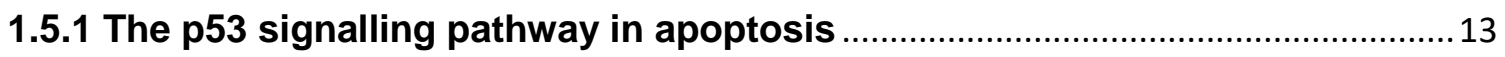

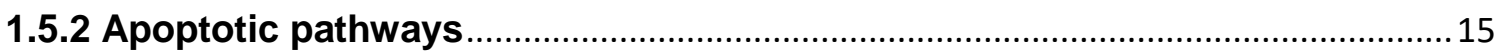

1.6 Senescence: a major contributor to therapy resistance.......................................... 18

1.7 The tumour microenvironment (TME) contributes to drug resistance ...................22

1.8 The controversial role of autophagy in cancer development .................................23

1.9 High mobility group box 1 promotes an inflammatory state in cancer

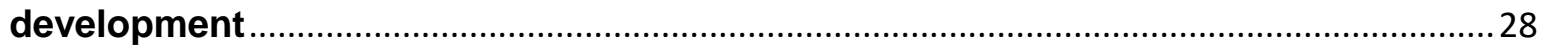

1.10 Autophagy contributes to DXR resistance in cancer ............................................ 30

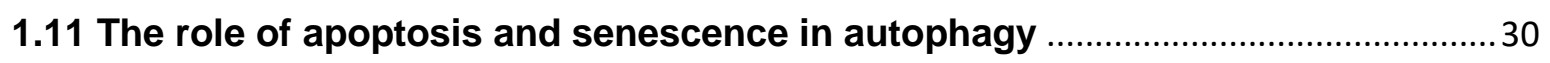

1.12 The role of autophagy in the tumour microenvironment (TME) ............................. 32

1.13 Inhibition of autophagy can enhance cancer cell death ......................................... 33

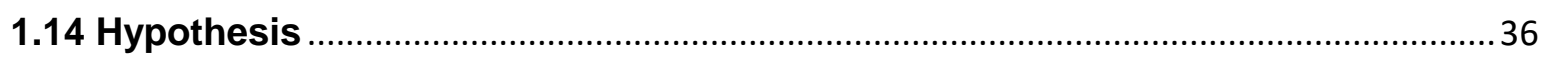

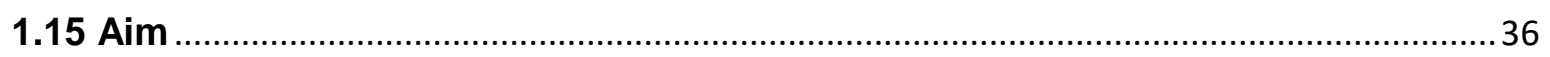

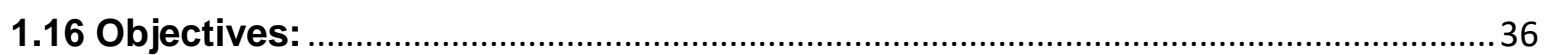

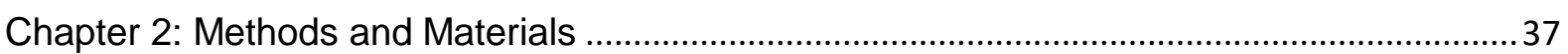

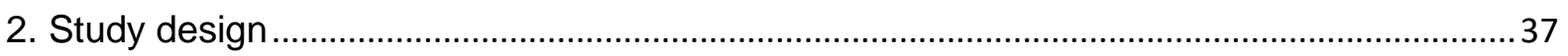

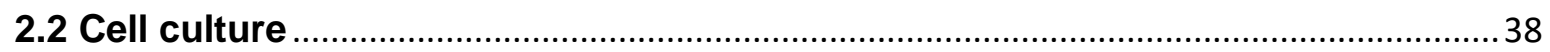




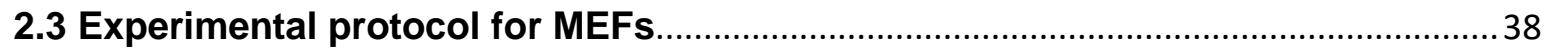

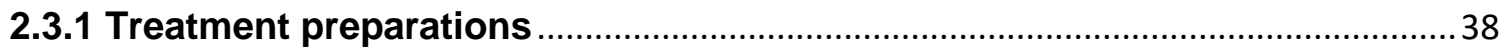

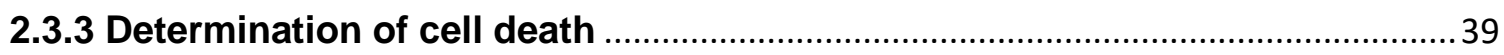

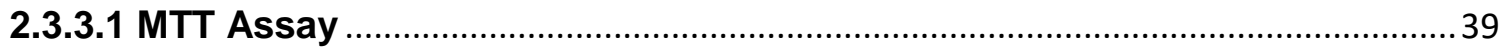

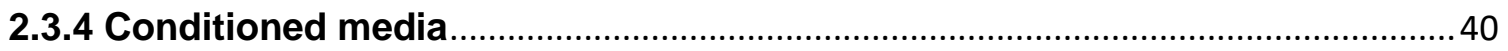

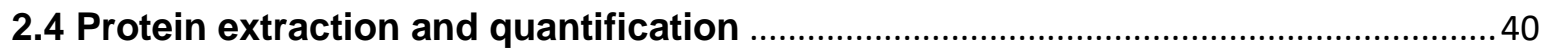

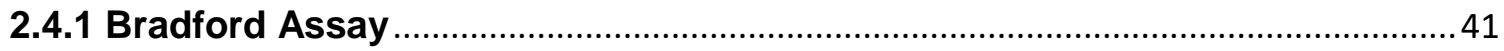

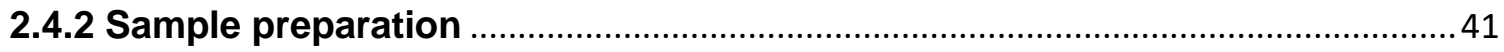

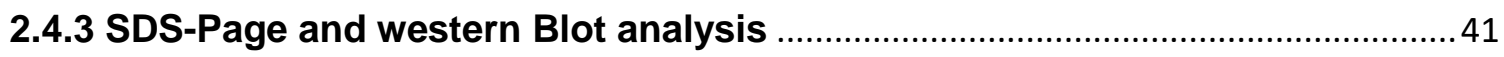

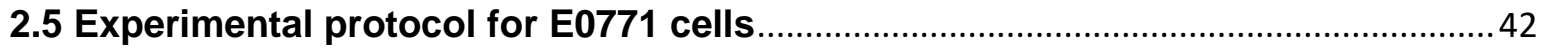

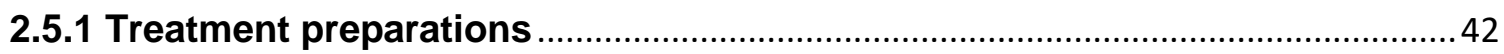

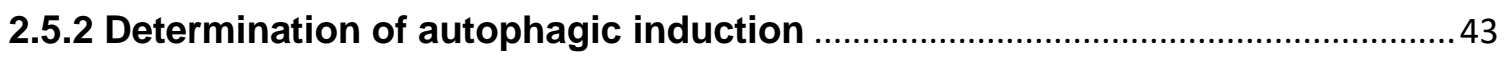

2.5.3 Western blot analysis for autophagy and proliferation .................................... 45

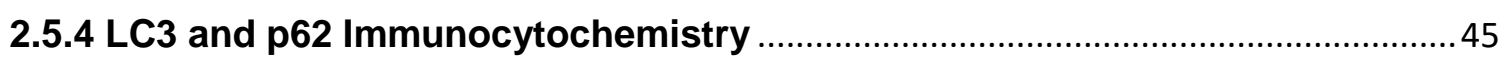

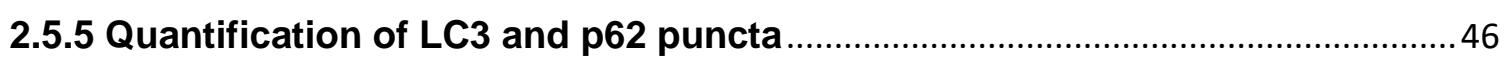

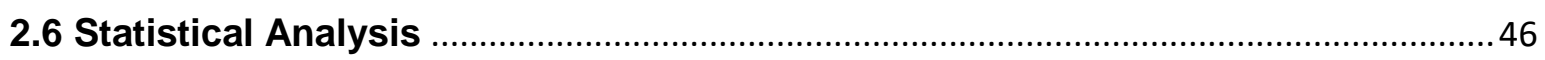

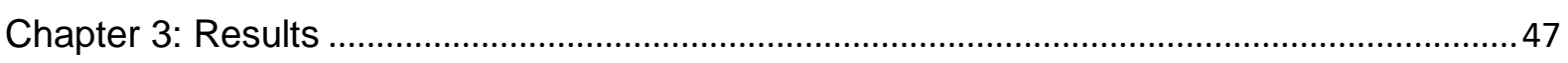

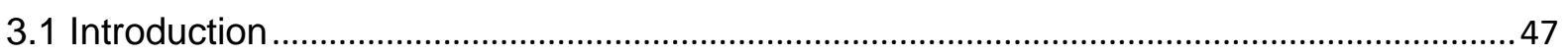

3.1.1 Dose response changes in cell viability following DXR treatment .................. 47

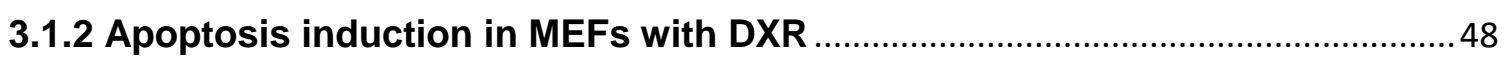

3.2 Determination of senescence in MEFs after DXR treatment ....................................53

3.2.1 Senescence associated B-galactosidase assay ................................................ 53

3.2.2 Senescence induction in MEFs after DXR treatment..........................................5

3.3 Paracrine effects of senescent and apoptotic MEFs on E0771 cells......................61

3.3.1 Changes in cell viability following Conditioned media and DXR treatment...61

3.3.2 Apoptosis activation in E0771 cells following Conditioned media and DXR treatment

3.3.3 Senescence determination in E0771 cells after conditioned media and DXR treatment

3.3.4 Changes in cell viability following conditioned media and DXR treatment

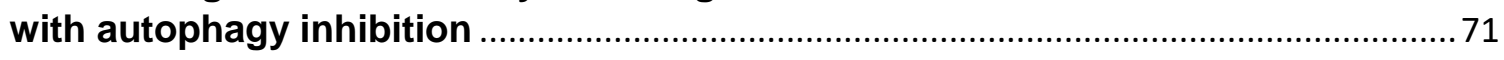

3.4 Autophagy Immunocytochemistry: Quantification of LC3 and p62 puncta ..........86

3.4.1 LC3 II stain following conditioned media and DXR treatment with autophagy inhibition.

3.4.1.1 Representative images for LC3 puncta quantification

3.4.2 p62 stain following conditioned media and Doxorubicin treatment with autophagy inhibition 
3.4.2.1 Representative images for p62 puncta quantification ................................... 90

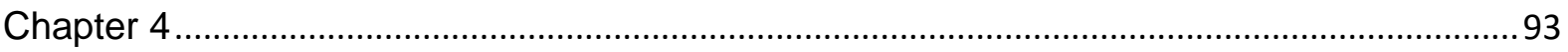

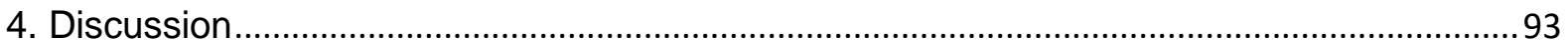

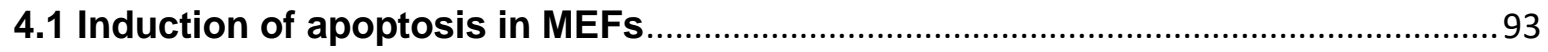

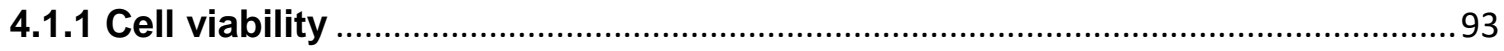

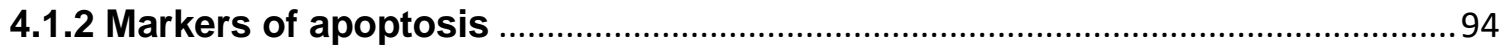

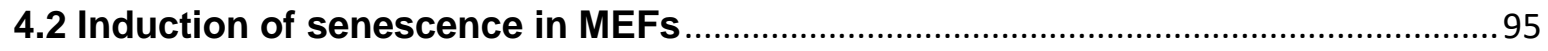

4.2.1 Senescence associated ß-galactosidase activity ............................................. 95

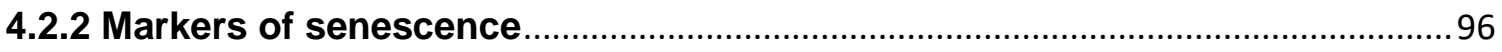

4.3 The paracrine effects of senescent and apoptotic MEFs on E0771 cells............. 98

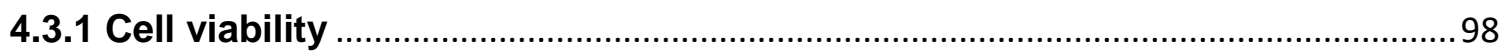

4.3.2 Markers of apoptosis following conditioned media treatment .......................... 98

4.3.3 Markers of senescence following conditioned media treatment ...................... 99

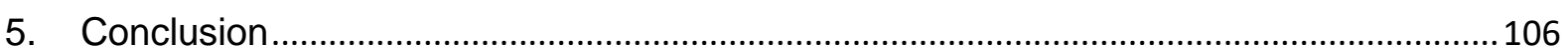

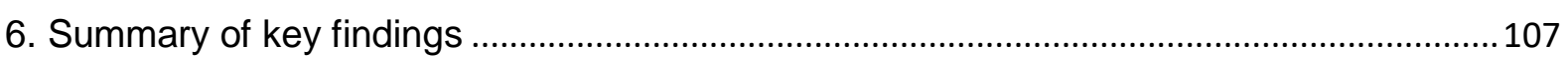

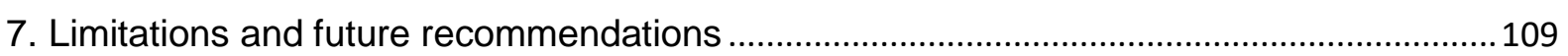

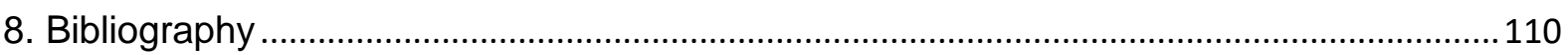

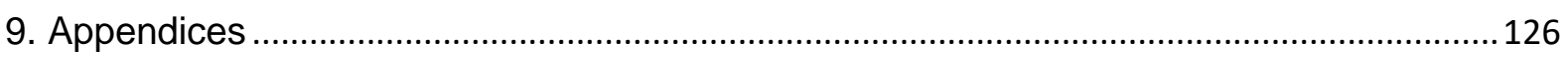

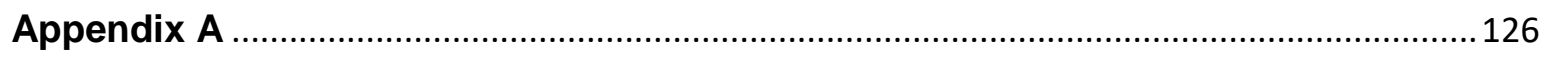

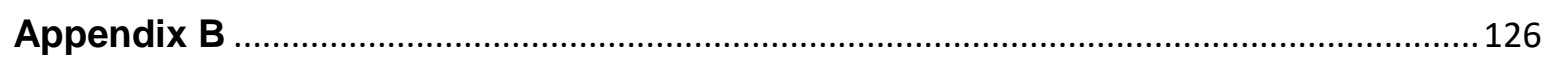

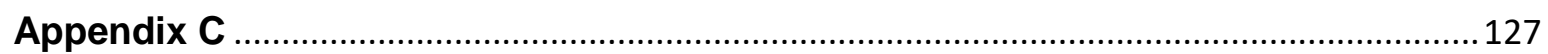

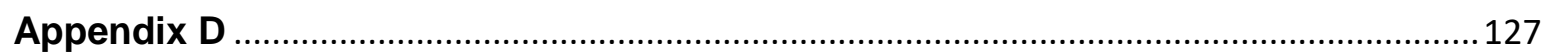

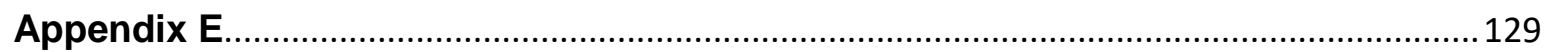

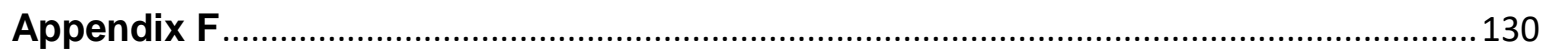

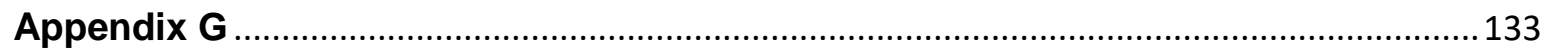

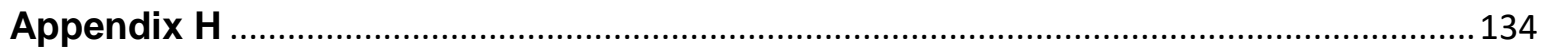




\section{List of Figures}

Figure 1.1: The PI3K/Akt signalling pathway and its downstream targets.

Figure 1.2: The PI3K/Akt/mTOR and the RAS/RAF/MEK/MAPK pathways frequently harbour oncogenic transformations in cancer.

Figure 1.3: The Ras/Raf/MEK/ERK signalling pathway.

Figure 1.4: Different types of stress signals activate the p53 protein, which enhances the role of $\mathrm{p} 53$ as a tumour suppressor gene.

Figure 1.5: The intrinsic and extrinsic mitochondrial pathways of p53 induced apoptosis.

Figure 1.6: The p53 pathway controls cellular senescence via p21 activation.

Figure 1.7: The p16/RB pathway is a major role player in cell cycle control.

Figure 1.8: The controversial role of autophagy in cancer.

Figure 1.9: The autophagy process.

Figure 1.10: Autophagy promotes cancer cell survival by protecting the cells from oxidative stress, ROS, starvation and hypoxia which allows the cancer cells to develop chemoresistance and continue growing.

Figure 2.1: Study design.

Figure 3.1: Percentage viability of MEFs after a DXR treatment period determined by an MTT assay.

Figure 3.2: Western blot analysis to detect Caspase 8 cleavage.

Figure 3.3: Western blot analysis to detect Caspase 9 cleavage.

Figure 3.4: Western blot analysis to detect Caspase 3 cleavage.

Figure 3.5: Western blot analysis to detect PARP cleavage.

Figure 3.6: Phenotypical changes in MEFs following treatment with DXR for senescence induction.

Figure 3.7: Western blot analysis to detect MCM2 expression.

Figure 3.8: Western blot analysis to detect p53 expression.

Figure 3.9: Western blot analysis to detect p21 expression. 
Figure 3.10: Western blot analysis to detect p16 expression.

Figure 3.11: Western blot analysis to detect RB expression.

Figure 3.12: Percentage viability of E0771 cells after a conditioned media and DXR treatment period determined by an MTT assay.

Figure 3.13: Percentage viability of E0771 cells after conditioned media and DXR treatment period with autophagy inhibition determined by an MTT assay.

Figure 3.14: Western blot analysis to detect Caspase 9 cleavage in E0771 cells.

Figure 3.15: Western blot analysis to detect Caspase 3 in E0771 cells.

Figure 3.16: Western blot analysis to detect PARP cleavage in E0771 cells.

Figure 3.17: Western blot analysis to detect MCM2 in E0771 cells.

Figure 3.18: Western blot analysis to detect p53 in E0771 cells.

Figure 3.19: Western blot analysis to detect p16 in E0771 cells.

Figure 3.20: Western blot analysis to detect RB in E0771 cells.

Figure 3.21: Percentage viability of E0771 cells after conditioned media and DXR treatment period with autophagy inhibition determined by an MTT assay.

Figure 3.22: Western blot analysis to detect LC3 II in E0771 cells.

Figure 3.23: Western blot analysis to detect p62 in E0771 cells.

Figure 3.24.1: Western blot analysis to detect phosphorylated Akt in E0771 cells.

Figure 3.24.2: Western blot analysis to detect total Akt in E0771 cells.

Figure 3.24.3: $\mathrm{P}$. Akt/total Akt in E0771 cells.

Figure 3.25.1: Western blot analysis to detect phosphorylated ERK in E0771 cells.

Figure 3.25.2: Western blot analysis to detect total ERK 1 and ERK 2 in E0771 cells.

Figure 3.25.3: P.ERK 1/total ERK 1 in E0771 cells.

Figure 3.25.4: P.ERK 2/total ERK 2 in E0771 cells.

Figure 3.26.1: Immunocytochemistry analysis of LC3 expression in E0771 cells.

Figure 3.26.1.1: Representative images of LC3 puncta quantification in E0771 cells following $\mathrm{CM}$ and DXR treatment and autophagy inhibition. 
Figure 3.26.2: Immunocytochemistry analysis of p62 expression in E0771 cells.

Figure 3.26.2.1: Representative images of LC3 puncta quantification in E0771 cells following $\mathrm{CM}$ and DXR treatment and autophagy inhibition.

Figure 6.1: A summary of the key findings.

Figure 6.2: Akt and ERK contributes to cancer cell proliferation and DXR treatment resistance. 


\section{List of Tables}

Table 2.1: MEFs were categorized into three groups and were treated accordingly to generate conditioned media.

Table 2.2: Antibodies used in western blot analysis for MEFs.

Table 2.3: E0771 cells were allocated into 6 groups and treated with conditioned media generated from MEFs.

Table 2.4: E0771 cells were categorized into 12 groups and treated with conditioned media generated from MEFs as well as Bafilomycin to determine autophagic flux.

Table 2.5: Antibodies for autophagy and proliferation used in western blot analysis for E0771.

Table 3.1: The average number of senescent cells present in a randomly selected cell population (MEFs). 


\section{List of Abbreviations}

\section{Units of measurement}

${ }^{\circ} \mathbf{C}$

degrees Celsius

$\mathrm{cm}^{2}: \quad$ centimetre squared

kDa: $\quad$ kilo Dalton

$\mu \mathrm{M}: \quad$ micro Molar

ml: millilitre

mm: millimetre

$\mathrm{mg} / \mathrm{ml}: \quad$ milligram per millilitre

$\mu l: \quad$ microlitre

nm: nanometre

nM: nanomolar 
ADCD:

Akt:

APAF-1:

ATG13:

B

Bad:

Bax:

Bcl-2:

Bcl-xl:

BER:

Bid:

Bim:

$\underline{\mathbf{C}}$

CAF:

CDK:

CM:

CM-C:

CM-C-DXR:

CM-A:

CM-A-DXR:

CM-S:

CM-S-DXR:

DXR
Autophagy related cell death

Serine/threonine kinase (Protein kinase B)

Apoptotic protease activating factor 1

Autophagy related (ATG) gene 13

Bcl-2 antagonist of cell death

Bcl-2 associated $\mathrm{X}$ protein

B-cell lymphoma protein 2

B-cell lymphoma-extra large

Base extension repair

$\mathrm{BH} 3$ interacting domain death agonist

Bcl-2-like protein 11

Cancer associated fibroblasts

Cyclin dependent kinase

Conditioned media from fibroblasts

Conditioned media from fibroblasts untreated (Control)

Conditioned media from fibroblasts treated with DXR

Conditioned media from apoptosis-induced fibroblasts untreated

Conditioned media from apoptosis-induced fibroblasts treated with DXR

Conditioned media from senescence-induced fibroblasts untreated

Conditioned media from senescence-induced fibroblasts treated with 
$\mathrm{CO}_{2}: \quad$ Carbon Dioxide

$\underline{\mathbf{D}}$

DAMP: Damage associated molecular pattern

dATP: Deoxyadenosine triphosphate

DISC: $\quad$ Death inducing signalling complex

DNA: Deoxyribonuclease

DMEM: $\quad$ Dulbecco's Modified Eagle's medium

DXR: Doxorubicin

E

ER: $\quad$ Estrogen receptor

ERK: $\quad$ Extracellular signal-regulated kinase

E2F-1: $\quad$ E2F transcription factor 1

F

FBS: $\quad$ Fetal Bovine Serum

$\underline{\mathbf{G}}$

GTP: Guanosine-5'-triphosphate

브

HER2: Human epidermal growth factor receptor-2

HMGB1: $\quad$ High motility group box 1

I

IGF: Insulin-like growth factor

IGFBP: IGF-binding proteins

IL-1: Interleukin-1

IL-1ß: Interleukin-1 beta 
IL-6: $\quad$ Interleukin-6

$\underline{\mathbf{L}}$

LC3: $\quad$ Microtubule-associated protein 1A/1B light chain 3B

LIR: $\quad$ LC3 interacting region

$\underline{M}$

MAPK: $\quad$ Mitogen-activated protein kinase

MCL-1: Induced myeloid leukemia cell differentiation protein

MCM: Mini chromosome maintenance proteins

MCM2: $\quad$ Mini chromosome Maintenance Complex Component 2

MDR1: multi-drug resistance gene

MEFs: $\quad$ Mouse Embryonic Fibroblasts

MEK: $\quad$ Mitogen-activated protein kinase

MMP 2: $\quad$ Matrix metalloproteinase 2

MPT: $\quad$ Mitochondrial permeability transition

MOMP: $\quad$ Mitochondrial outer membrane permeability

mTOR: $\quad$ Mammalian target of rapamycin

mTORC1: $\quad$ Mammalian target of rapamycin complex 1

mTORC2: Mammalian target of rapamycin complex 2

$\underline{\mathbf{N}}$

NFkB: $\quad$ Nuclear factor kappa beta

NLS: $\quad$ Nuclear localization signal

$\underline{\mathbf{P}}$

pAkt: Phosphorylated Akt 
PARP: $\quad$ Poly (ADP-ribose) polymerase

PB1: $\quad$ Phox-BEM1 domain

PBS: $\quad$ Phosphate Buffered Saline

PCNA: Proliferating cell nuclear antigen

pERK: $\quad$ Phosphorylated ERK

PIP $_{2}$ Phosphatidylinositol bisphosphate

$\mathbf{P I P}_{3}$ : $\quad$ Phosphatidylinositol triphosphate

PI3K: $\quad$ Phosphatidylinositol 3-kinase

PR: $\quad$ Progesterone receptor

PRR: $\quad$ Pattern recognition receptors

PTEN: $\quad$ Phosphatase and tensin homolog

$\underline{\mathbf{R}}$

RAGE: $\quad$ Receptor for advanced glycation end products

RB: $\quad$ Retinoblastoma

RNA: $\quad$ Ribonucleic acid

ROS: $\quad$ Reactive Oxygen Species

RPM: $\quad$ Revolutions per minute

$\underline{\mathbf{S}}$

SA-ß-gal: $\quad$ Senescence associated-B-galactosidase

SASP: $\quad$ Senescence associated secretory phenotype

SDF-1: $\quad$ Stromal cell derived factor 1

SQSTM 1: $\quad$ Sequestosome-1 
TAMs: $\quad$ Tumour associated macrophages

tBid: $\quad$ membrane-targeted death ligand

TLR2: $\quad$ Toll like receptor 2

TLR4: $\quad$ Toll like receptor 4

TGF- $\beta$ : $\quad$ Transforming growth factor

TIS: $\quad$ Therapy induced senescence

TME: $\quad$ Tumour microenvironment

TNF- $\alpha$ : Tumour necrosis factor

TNF-R: $\quad$ Tumour necrosis factor receptor

TNBC: $\quad$ Triple negative breast cancer

TRAIL: $\quad$ Tumor necrosis factor-related apoptosis-inducing ligand

$\underline{\mathbf{U}}$

UBA: $\quad$ Ubiquitin associated domain

UV: $\quad$ Ultra-violet

$\underline{\mathbf{X}}$

X-gal: $\quad$ 5-bromo-4-chloro-3-indolyl- $\beta$-D-galactopyranoside

$\underline{Y}$

YB-1: $\quad$ Y-box binding protein 1 


\section{Chapter 1: Literature Review}

\subsection{Breast cancer: Introduction}

Breast cancer frequently occurs in women in both developed and developing countries and therefore creates a major financial and health burden throughout the world (Desantis et al., 2016). The heaviest global burden is experienced in developed countries, but the incidence in developing countries are rapidly increasing. Although many factors contribute to the prevalence, risk and distribution of cancer, many are associated with socioeconomic development (Caldwell, 2001; Bray et al., 2018). With the rapidly growing populations, one in ten newly diagnosed cancers each year, will be breast cancer (Siegel et al., 2018).

Worldwide, one out of four women will be diagnosed with breast cancer during their lifetime (Bray et al., 2018). Out of 36 different types of cancers worldwide, lung cancer is the leading cause of death among women, followed by breast cancer (Torre et al., 2017; Bray et al., 2018). However, breast cancer is the most frequently diagnosed cancer in most countries worldwide (154 countries out of 185). Assuredly, the use of chemotherapy has decreased the death rate specifically for breast cancer by 25\%, since 1990 (Parton et al., 2001). However, over the last three decades, global breast cancer incidence has increased annually by $3.1 \%$ and during 2010, 1.6 million cases of breast cancer were reported (Forouzanfar et al., 2011; Looi et al., 2013). Shockingly, the most recent data indicates that approximately 2.1 million breast cancer cases were reported during 2018 and 600000 cases resulted in death (Bray et al., 2018). In South Africa, $22 \%$ of the female population was diagnosed with breast cancer during 2013 and considering that South Africa is categorized as a developing country, breast cancer incidence is alarmingly high and continues to increase annually since 2008 (NCR, 2013).

Different gene mutations can result in the onset of different subtypes of breast cancer which makes it more difficult to determine the exact diagnosis as well as treatment options that will be most successful to eliminate the cancer (Sims, 2007). Breast cancer can be categorized in many ways, including tumour marker expression, clinical features and its histological type ( $\mathrm{Li}$, Uribe \& Daling 2005; Gudavicience et al., 2005). The breast mainly consists of lobular (also sometimes referred to as glandular) tissue, ductal cells and stromal tissues (Sharma et al., 2010). Lobular tissue contains milk producing glands, ductal cells form small tubes that are responsible for transporting milk towards the nipple and stromal tissues are constituted of fatty and fibrous connective tissues. Different types of tumours can therefore develop in different areas of the breast. To further contribute to the complexity of cancer, breast cancer can also 
be characterized as non-invasive or invasive (Pareja et al., 2016). In non-invasive breast cancer, the cancerous cells are unable to invade surrounding connective tissues within the breast because they are confined within the breast milk ducts. The form of non-invasive breast cancer that occurs the most is ductal carcinoma in situ which indicates that the cells that line the milk ducts have become cancerous. Invasive breast cancer occurs once the cells break through the lobular wall (milk producing glands) and invades the surrounding fatty and connective tissue in the breast. Lobular carcinoma in situ (LCIS) is invasive and is characterized by a significant increase in the number of cells within the milk glands. LCIS is also the type of breast cancer that occurs most frequently. The incident rates of lobular carcinomas in the United States are increasing at an alarming rate when compared to ductal carcinomas ( $\mathrm{Li}$ et al., 2003). The percentage of lobular carcinoma has increased by $65 \%$ from 1987 to 1999, while ductal carcinoma has only increased by 3\% (Arpino et al., 2004).

Immunohistochemical subtyping for estrogen receptor (ER), human epidermal growth factor receptor-2 (HER2) and progesterone receptor (PR) further divides breast cancer into 4 molecular subtypes: Luminal A (ER and/or PR positive and HER2 negative), Luminal B (ER and/or PR positive and HER2 positive or negative), Triple negative breast cancer (TNBC) (Hormone receptor negative) and HER2 overexpression (Hormone receptor negative and HER2 positive) (Wang, 2017). TNBC is considered to be an aggressive form of breast cancer because the cancer often returns after a few years and presents in $10 \%$ to $20 \%$ of breast cancer cases (Kumar \& Aggarwal, 2008; Pareja et al., 2016). It does not respond to hormonal therapy treatments because the cells confined within the tumour are negative for progesterone, estrogen and HER2 receptors. In 2009 it was reported that 500000 women die every year because of breast cancer and 150000 (30\%) of these cases are diagnosed with TNBC (Kumar \& Aggarwal, 2008).

Several studies have focused on and examined the clinical and pathological differences between ductal and lobular carcinomas, while less attention is given to rarer types of breast cancer which account for $10 \%$ of all cases (Li et al., 2005; Gudaviciene et al., 2015). These types of breast cancer include:

- Mucinous carcinoma of the breast: This carcinoma contains increased amounts of extracellular mucus that is in direct contact with the stroma. Most of the tumour mass is constituted of acellular mucus and not neoplastic cells. 
- Medullary carcinoma: This carcinoma forms a spherical tumour mass and is constituted of lymphocytes and poorly differentiated epithelial cells.

- Metaplastic carcinoma: The carcinoma is constituted of cells that are normally not found within the breast.

- Inflammatory breast cancer: It is a rapidly growing form of breast carcinoma associated with thickening and reddening of the skin over $50 \%$ of the breast and is not always associated with tumour development.

- Metastatic cancer: The cancer cells have spread beyond the breast tissue and often infiltrate into brain, lungs and bone tissue.

It is therefore important to evaluate all the characteristic differences between carcinoma types to be able to understand the nature of the tumour, etiologies and clinical features of different types of breast cancers (Li et al., 2005).

The risk factors for breast cancer often include: previous cancer history, family history of cancer, hormonal changes as well as dietary and lifestyle factors (Cauchi et al., 2016). The most common symptom for breast cancer is a lump in the breast or armpit region. In advanced or metastatic stages, bone pain, shortness of breath, loss of appetite and weight loss can occur. A lobule biopsy is most commonly used to diagnose breast cancer (Li et al., 2005).

Breast cancer accounts for $25 \%$ of all cancer cases worldwide and is not limited to females; approximately $1 \%$ of breast carcinomas occur in the male population (Giordano et al., 2004; Torre et al., 2012). Survival rates vary, but increased survival rates are expected to occur each year which can be attributed to various breakthroughs that have been made in early diagnosis of breast cancer, screening methods and the development of new treatment strategies (Nounou et al., 2015).

\subsection{Treatment strategies for breast cancer}

Although the current standard treatment for breast cancer patients is radiation, surgery and chemotherapy or a combination of surgery with chemotherapy, the therapeutic options for breast cancer treatment depends on the biological characteristics of the tumour (Amornsupak, et al., 2014). Hormone therapy is often recommended for a low-grade, ER positive tumour whereas chemotherapy is administered depending on the hormonal status of the tumour and 
is generally only administered to high grade tumours (Lovitt, Shelper \& Avery, 2018). Early diagnosis of breast cancer generally increases the chance of survival, but the unresponsiveness of cancer cells to chemotherapeutics is still a major concern where the response rate to chemotherapeutics is estimated to be only $50 \%$ (Rampurwala, Rocque \& Burkard, 2014). In addition, it has been reported that about $30 \%$ of early stage breast cancer patients are at risk for the development of drug resistance and cancer recurrence (Chang, 2012). Triple negative breast cancer (TNBC) is the most diagnosed type of breast cancer and therefore has the highest mortality rate (Aydinlik et al., 2017). Treatment failure generally occurs when cancer cells become resistant to chemotherapeutic agents, which leads to relapse, metastasis and ultimately death, despite the continuous administration of anti-cancer drugs (Housman et al., 2014).

During the 1960's, Doxorubicin (DXR) was introduced as an anti-cancer therapy (a chemotherapeutic) and is currently one of the most potent broad-spectrum anti-tumour antibiotics commonly used to treat breast, lung and ovarian cancer (Fujita et al., 2011; Osman et al., 2013). DXR (also known as Adriamycin) belongs to the anthracycline family and was first isolated from a soil bacterium, Streptomyces peucetius (Yang \& Teves, 2015). DXR contains both $p$-quinone and $p$-hydroquinine structures which allows for the production of free radicals through cytochrome $\mathrm{P} 450$ mediated reduction and iron-mediated oxidation of DXR. NADPH allows DXR to undergo a one-electron reduction of the $p$-quinone to form a semiquinone radical to produce free hydroxyl radicals, which is responsible for DNA damage (Mizutani et al., 2003).

DXR is commonly used to treat breast, bladder, lung and ovarian cancer, as well as soft tissue sarcoma, Hodgkin's lymphoma and Kaposi's sarcoma (Momparler et al., 1976; Rivankar, 2014). Many studies have indicated that DXR shows great efficacy in the killing of cancer cells, in solid tumours as well as in blood cancers like leukemia (Yang \& Teves, 2015), however, drug resistance and possible side effects including heart muscle damage limits the success of the treatment (Thorn, 2011).

Various mechanisms for DXR-mediated cell death have been proposed, including (1) topoisomerase II poisoning, (2) oxidative stress and (3) the formation of DNA adducts (Yang et al., 2014). However, some mechanisms such as DNA and RNA synthesis inhibition can only be identified at dosages higher than the clinical dose ( 40 to $\left.60 \mathrm{mg} / \mathrm{m}^{2}\right)($ Gewirtz, 1999). 
Anthracyclines are classified as planar molecules which are able to intercalate between neighbouring DNA base pairs. Once DNA is topologically constricted, the double helix unwinds and produces DNA supercoils, which severely increases torsional stress (Yang et al., 2014).

\subsubsection{Topoisomerase II poisoning}

Topoisomerases are evolutionary conserved from bacteria to humans and are used to facilitate DNA transcription, regulation and synthesis. Topoisomerase II binds DNA supercoils and breaks the strands of one DNA duplex, while the other DNA strand is passed through the forming gap and reseals the break. A deficiency in topoisomerase II prevents cytokinesis and ultimately results in cell death (Carpenter et al., 2004). Anti-cancer drugs like DXR specifically target topoisomerases to kill tumour cells. DXR poisons topoisomerase II by diffusing into the nucleus where it intercalates with DNA.

\subsubsection{Oxidative stress}

The addition of one electron to the quinone structure of DXR can readily form a semiquinone radical, which is able to react with oxygen and produce reactive oxygen species (ROS) (Myers, 1998). Once the reaction is completed, superoxide and hydrogen molecules are generated, leading to severe DNA damage. The sudden increase in ROS can also stimulate the onset of lipid peroxidation (the oxidative degradation of lipids) as well as the induction of oxidative stress. Furthermore, DXR is also an iron chelator and can convert hydrogen peroxide into highly reactive hydroxyl radicals (Myers et al., 1998). The release of DXR induced free radicals can thus potentially cause oxidative damage which will result in DNA damage and cell death. It was indicated in a study that slow intravenous infusion of DXR $(0.1 \mu \mathrm{M})$ into the plasma after 72 to 96 hours resulted in a 4 -fold increase of DNA base oxidation in breast cancer patients (Doroshow et al., 2001).

\subsubsection{DNA adduct formation}

The target site for DXR intercalation in DNA is adjacent to guanine-cytosine base pairs (Livraghi \& Garber, 2015). The formation of DNA adducts activates the DNA damage response and leads to cell death, independently of topoisomerase II. The formation of DNA adducts after DXR intercalation is identified at clinically applicable drug concentrations, which suggests that DXR-DNA adducts are formed during chemotherapeutic treatment (Taatjies et al., 1997). 
The mechanism of action of DXR and many anthracycline drugs have been intensively studied, but the exact mechanisms for cell death remain controversial (Yang et al., 2014). Therefore, by understanding the mechanism of DXR-mediated cell death, future treatment strategies can be developed to enhance cell death of cancer cells and to minimise side effects induced by chemotherapeutic treatments.

\subsection{Development of treatment resistance}

In general, treatment failure can be contributed to chemotherapeutic resistance in more than $90 \%$ of patients with metastatic cancer and failure following DXR treatment is also subjected to these statistics. Once the cells are exposed to DXR, the stress responsive $Y$-box binding protein 1 (YB-1) translocates to the nucleus to induce the expression of the multidrug resistance gene 1 (MDR1) (Chen \& Simon, 2000). The MDR1 gene encodes the PGlycoprotein to export substances to the outside of the cell to protect cells from toxic substances (Dresher, 2002). High levels of MDR1 could therefore limit the ability of DXR to infiltrate into cancer cells, which promotes DXR resistance and increased cell growth (Yang et al., 2010). Similarly, Shen et al demonstrated that MDR1-transduced human MDA-MB-435 (MDR) breast cancer cells are 9-fold more resistant to DXR than wild type MDA-MB-435 cells. DXR uptake in the nucleus of MDR cells was 2-fold lower when compared to the wild type cells (Shen et al., 2008).

Exposure to DXR induces severe DNA damage which can induce point mutations and single and double strand breaks (Weil and Chen, 2012). Cells are able to employ various DNA repair mechanisms, including base extension repair (BER), homologous recombination (HR) and mismatch repair to restore damages on a regular basis (Sirbu et al., 2013). The ability of injured cells to induce DNA repair for survival is optimal for normal cells, but this repair process also allows tumour cells to undergo repair after chemotherapy or radiation.

Novel anti-cancer therapies include agents that target specific molecular defects in cancer cells to increase treatment efficacy and reduce toxicity (Livraghi \& Garber, 2015). Poly (ADPribose) polymerases (PARPs) respond to DNA damage caused by DXR where PARP- 1, -2 and -3 are classified as DNA damage-dependent PARPs. PARP proteins have enzymatic and scaffolding properties that can recruit DNA repair proteins and PARP 1 and 2 is responsible for BER functioning, to repair single strand DNA breaks (Weil and Chen, 2012). The inhibition 
of BER can therefore eventually lead to cell death. PARP activation is driven by DNA damage and is involved in post-transcriptional modification of nuclear proteins (Livraghi \& Garber, 2015). In human cells, PARP-1 recruitment and activation is the first response to DNA damage caused by DXR (Mortusewicz et al., 2007).

Furthermore, Guo et al suggested that there are four possible fates for DXR once it enters MCF-7 breast cancer cells (Guo et al., 2016):

1. Cytoplasmic aldo-keto reductases metabolize DXR into a smaller toxic metabolite (13-OHdoxorubicinol).

2. It is taken up and isolated in lysosomes.

3. It binds to mitochondrial DNA before reaching the nucleus and induces oxidative stress in mitochondria, where after severely damaged and dysfunctional mitochondria are marked for degradation through the process of mitophagy.

4. Higher dosages of DXR are removed from the cancer cells via drug transporters.

These mechanisms could potentially explain why only small amounts of DXR is found in the nucleus of cancer cells (Guo et al., 2016).

Phosphoinositide 3-kinase (PI3K) dependent phosphorylation of downstream effector and transducer proteins is an important signalling pathway to determine cell fate after exposure to DNA damaging agents, like DXR (Harper \& Elledge, 2007). The DNA damage response is a signalling cascade that mediates the response to cellular damage by inducing DNA repair, cell cycle arrest and, if necessary, activating apoptosis. As mentioned previously, DNA damage causes DNA strands to break, which inactivates pathways mediated by PI3K related protein kinases (Cruet-Hennequart et al., 2012).

\subsection{The Phosphoinositide 3-kinase pathway in cancer}

The phosphoinositide 3-kinases (PI3Ks) are defined as a group of lipid kinases that biochemically phosphorylate the 3-hydroxyl group of phosphoinositides (Cantley, 2002). Class IA PI3Ks are heterodimers made up of a catalytic subunit (p110) and a regulatory subunit (p85) and is often deregulated in cancer (Baselga, 2011). The PI3K pathway is activated once ligands or growth factors bind the receptor tyrosine kinase (RTK) on the cell surface. Once the receptors are activated, phosphatidylinositol bisphosphate $\left(\mathrm{PIP}_{2}\right)$ is phosphorylated to 
phosphatidylinositol triphosphate $\left(\mathrm{PIP}_{3}\right)$ (Maehama \& Dixon, 1998). $\mathrm{PIP}_{3}$ acts as a binding site for Akt (a serine/threonine kinase) which is then phosphorylated at the cell membrane on Serine $^{473}\left(\mathrm{Ser}^{473}\right)$ by mammalian target of Rapamycin complex 2 (mTORC2) and on Threonine $^{308}\left(\mathrm{Thr}^{308}\right)$ by Pyruvate dehydrogenase kinase isoform 2 (PDK2) to stimulate cell growth and protein synthesis (Liu et al., 2009) (Figure 1.1). Akt is therefore involved in many downstream pathways that regulate cellular metabolism and proliferation and protects the cells from apoptosis (Kumar et al., 2013). However, hyperactivation of Akt often occurs in many cancers, which include breast (Chua et al., 2009) and prostate (Ayala et al., 2004) cancer, where it has been shown in tumour samples collected from breast and prostate cancer as well as leukaemia patients that mutations in the Akt signalling pathway is a regular occurrence (Sadeghi, 2012). Phosphate mediated Akt signalling is negatively regulated through the phosphatase activity of Phosphatase and tensin homolog (PTEN) that acts as a tumour suppressor (Waite et al., 2002; Xu et al., 2014). Once phosphatase activity is lost, $\mathrm{PIP}_{3}$ is not dephosphorylated to $\mathrm{PIP}_{2}$, allowing the phosphate molecule to be transported to Akt. This transfer then induces the expression of MDM2, BAD, mTOR and p21 to inhibit apoptosis activation, activate cell cycle progression and promote tumour growth by impairing cell cycle arrest at the G1 and G2 phases (Madrid et al., 2000; Kandel et al., 2002). The PI3K pathway therefore plays a major role in many cellular functions such as cell proliferation, cellular metabolism, differentiation and cell survival (Baselga, 2011). 


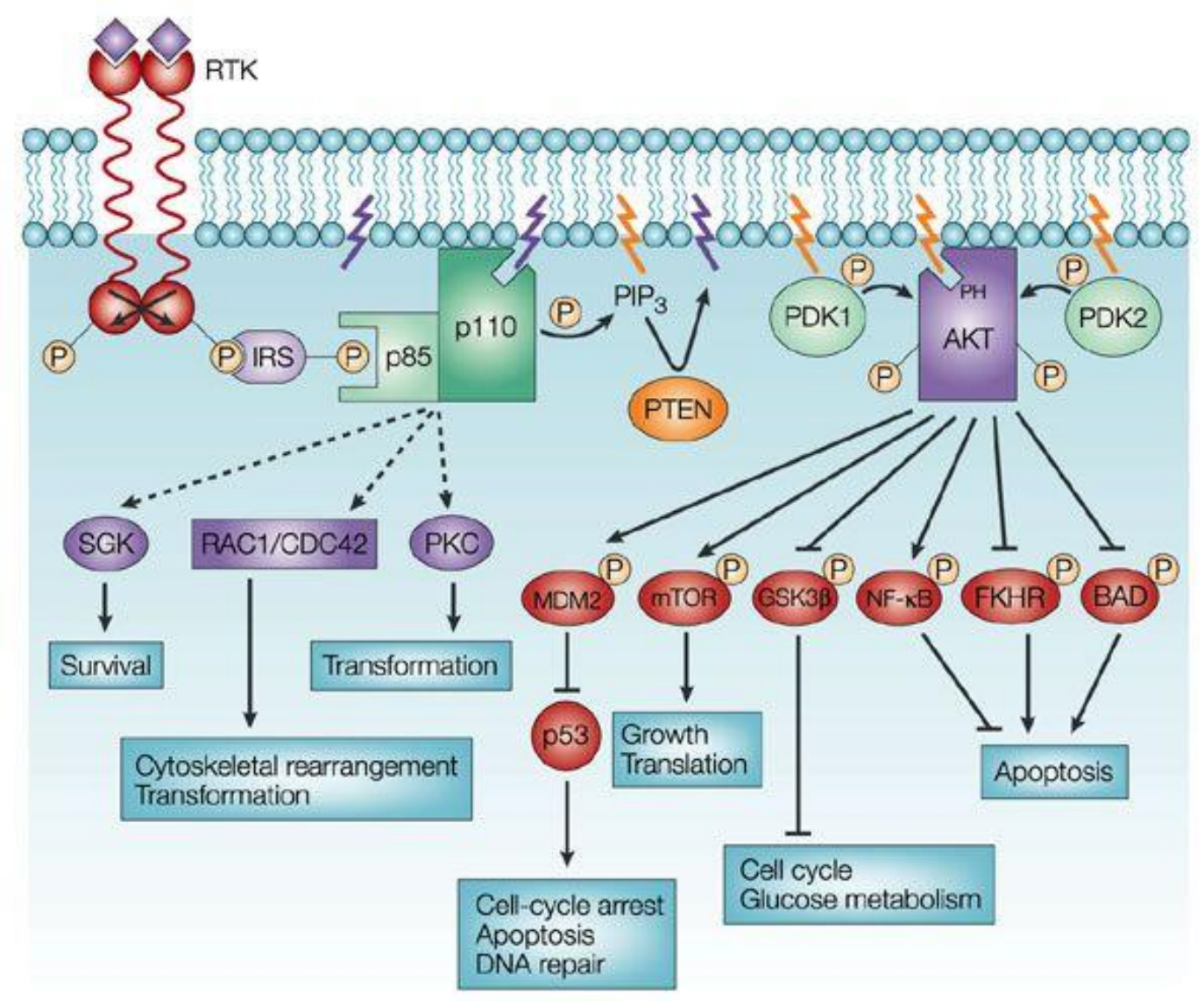

Figure 1.1: The PI3K/Akt signalling pathway and its downstream targets (from Sawyers \& Vivanco, 2002).

Genetic mutations in the PI3K pathway occur in $25 \%$ of breast cancer where most mutations occur in the PI3KCA gene, which encodes the p110a subunit and allows amino acids to be substituted in the kinase domain (exon 20) as well as the helical domain (exon 9) (StemkeHale et al., 2008; Baselga, 2011). Once the mutations occur, enzymatic functions are significantly increased which enhances the effects of downstream signalling components (like Akt) to promote oncogenic transformation (Figure 1.2) (Baselga, 2011). 


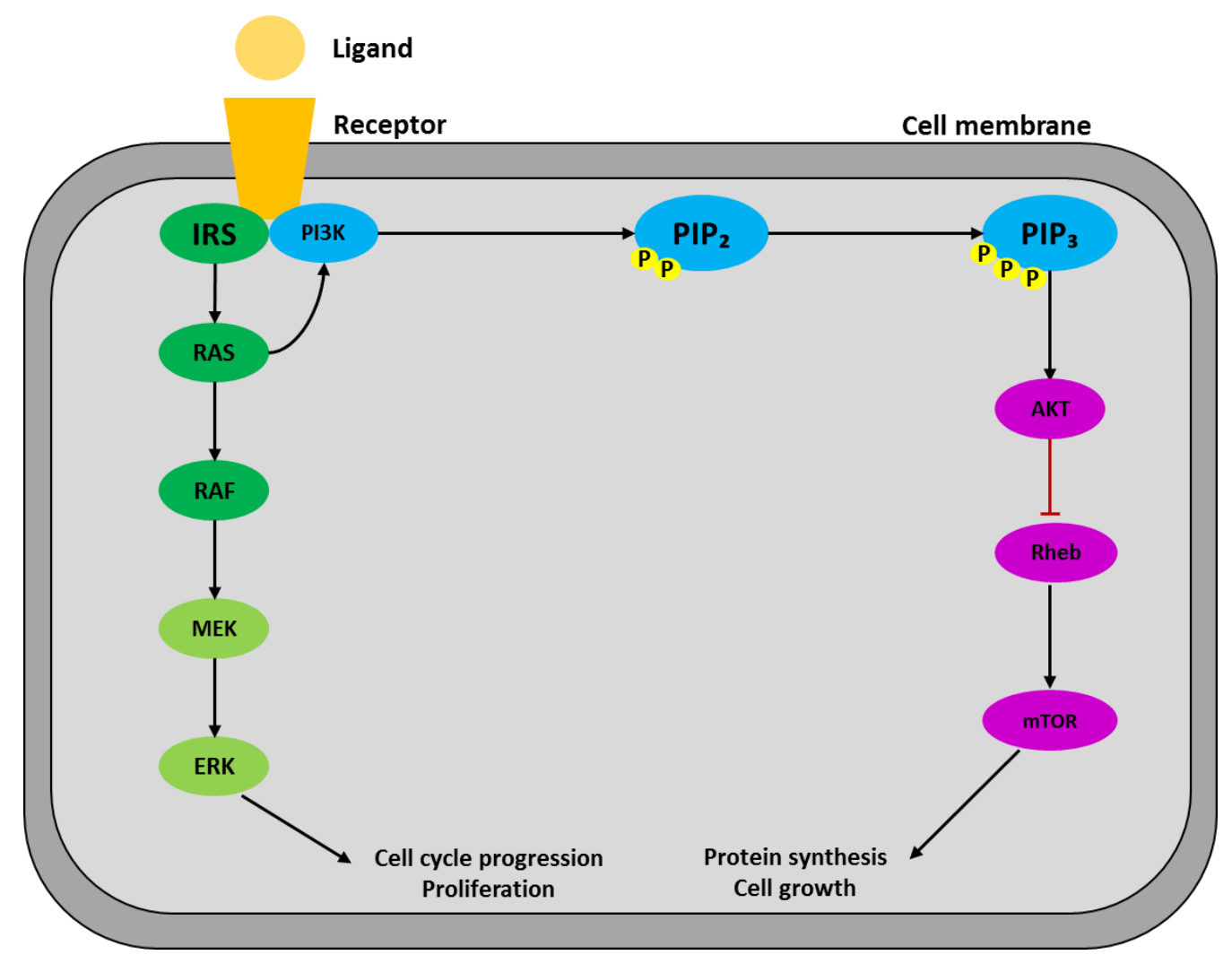

Figure 1.2: The $\mathrm{PI} 3 \mathrm{~K} / \mathrm{Akt} / \mathrm{mTOR}$ and the RAS/RAF/MEK/MAPK pathways frequently harbour oncogenic transformations in cancer (adapted from Toss and Cristofanilli, 2015).

The ability of PI3K to induce pro survival signalling is therefore linked with the activation of Akt but can also be dependent on Ras activation, which is part of the GTPases family. Approximately $25 \%$ of human tumours have mutations in a member of this family (Castellano \& Downward, 2011).

\subsubsection{Ras/Raf/MEK/ERK signalling contributes to pro-survival signalling of cancer cells}

The Ras-Raf-MEK-ERK pathway (MAPK/ERK pathway) is constituted of proteins that are located within the cell and functions to transport signals from the surface receptor to the cell's DNA to regulate gene expression (McCubrey et al., 2007). Ras is a GTP-binding protein and is generally the upstream molecule of many signalling pathways, including PI3K/Akt and Raf/MEK/ERK (Figure 1.2) (McCubrey et al., 2012). Once Ras is activated, the receptor tyrosine kinase effector, Raf (named for Rapidly Accelerated Fibrosarcoma), is recruited and phosphorylated at the cell membrane to stimulate serine-threonine kinase activity (Leicht et al., 2007). Activated Raf induces the activation and phosphorylation of Mitogen-activated 
protein kinase (MEK1/MEK2) and extracellular signal-regulated kinases (ERK1/2) (Robinson \& Cobb, 1997; Yoon \& Seger, 2006). Extracellular signal related-kinases are Serine/Threonine kinases and their activities are positively regulated by phosphorylation mediated by MEK. ERK then translocates to the nucleus to regulate transcription factors involved in cell proliferation and survival, which is mostly associated with ERK2 (Montagut \& Settleman, 2009). Once this pathway becomes dysregulated during cancer development, cellular growth arrest is inhibited, which will allow the cancer cells to rapidly proliferate (Hanahan \& Weinberg, 2000; Shields et al., 2000) (Figure 1.3).

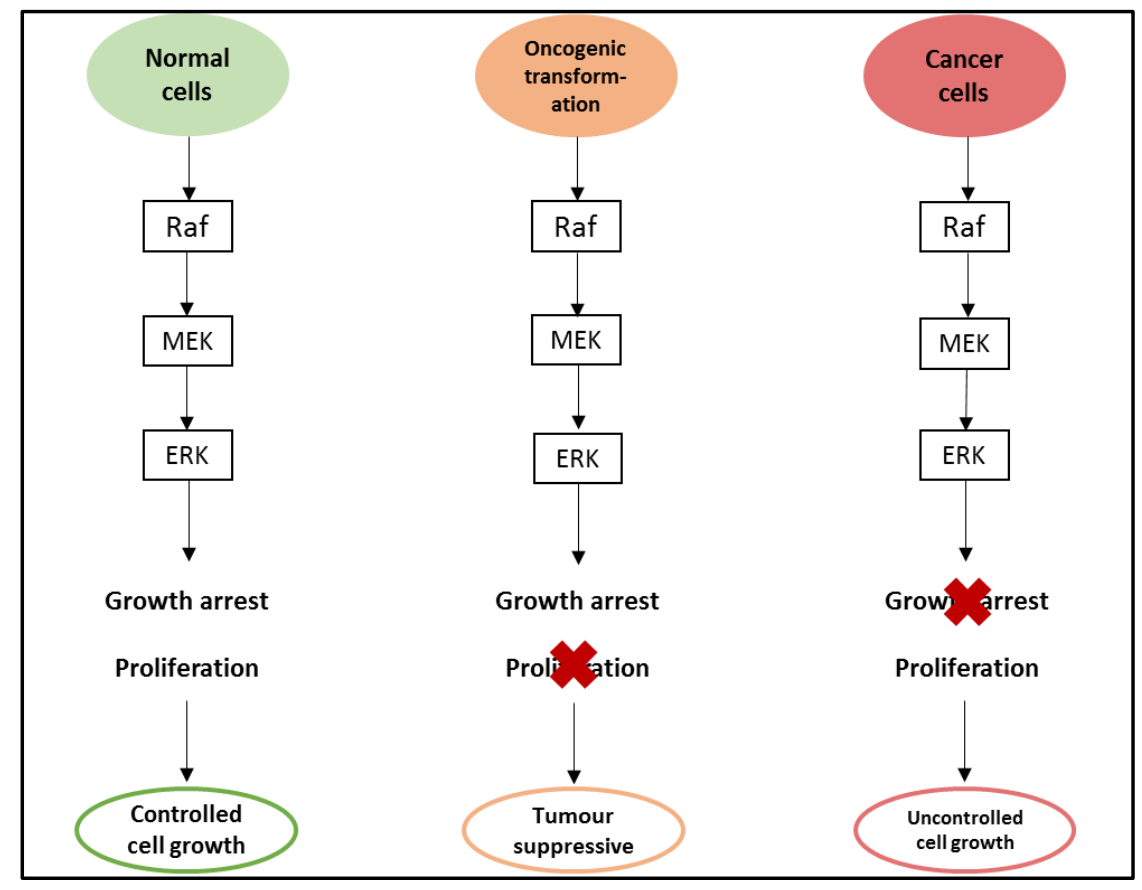

Figure 1.3: The Raf/MEK/ERK signalling pathway (adapted from Park, 2014).

The Raf/MEK/ERK pathway regulates many processes in cells such as apoptosis, differentiation and cell cycle progression. It inhibits the phosphorylation and inactivation of many apoptotic molecules including Bim, caspase 9, Bcl-2 and Bad (Steelman et al., 2004). It is therefore possible that cross-talk occurs between the PI3K/Akt and Raf/MEK/ERK pathways to ultimately prevent apoptosis from occurring.

Mutations at membrane receptors (Ras, PI3K, Akt) often cause this signalling pathway to become abnormally activated and occurs in approximately $20 \%$ of human neoplasms (Montagut \& Settleman, 2009). It is also suggested in many studies that the Raf/MEK/ERK pathway contributes to chemotherapeutic drug resistance in cancer cells. Abnormal activation of Raf induced resistance to both DXR and paclitaxel in breast cancer cells (Garnett et al., 
2004). Furthermore, Ras also has the ability to increase the expression of several molecules that are involved in cell cycle regulation including p53, p21 and p16. Ras therefore induces a permanent cell cycle arrest at the $\mathrm{G} 1$ phase, which is associated with a significant increase in the expression of the above-mentioned proteins (Serrano et al., 1997).

Ras proteins are considered to be molecular switches for various signalling pathways that regulate cell behaviour including cell proliferation and cell death (Shields et al., 2000). Tumour development and growth is highly dependent on the continued activation of Ras to avoid apoptosis, which is generally mediated by the activation of NFKB. However, some studies suggest that Ras induces pro- and anti-apoptotic signalling, depending on the cell type (Kaltschmidt et al., 2000; Ryan et al., 2000).

\subsection{Cell death: Apoptosis in cancer}

Apoptosis is a form of cell death that occurs during growth and development to maintain physiological homeostasis, with the ability to extensively delete cells with little tissue disruption and avoids the onset of inflammation (Kerr et al., 1972; Elmore, 2007). Uncontrolled tumour growth generally occurs when proliferation is enhanced and apoptosis is limited (Parton et al., 2001). Indeed, the biology or functioning of individual tumours can be described by examining the control and regulation of apoptosis at a molecular and biochemical level. Once these mechanisms are understood, individual tailored treatments can be developed to maximise the efficacy of treatment. A major method for apoptosis in situ detection and quantification is morphological assessment with either light or electron microscopy (Kerr et al., 1972; Doonan, 2008). Key features of apoptotic cells include cell shrinking, membrane blebbing and DNA fragmentation (Kerr et al., 1972; Elmore, 2007).

Carcinogenesis in epithelial tissue is characterized by defects in cellular functions, the accumulation of genetic mutations and rapid growth of cancer cells (Parton et al., 2001). It is believed that these processes can occur sometimes up to 30 years before the clinical onset of breast cancer (Kelloff et al., 2000). During premalignant stages, various biomarkers of the cell cycle are severely altered, specifically markers for proliferation and apoptosis. As soon as 24 hours after the onset of chemotherapy treatment, apoptosis is greatly increased in breast tumours and is commonly associated with reduced cell proliferation. Once the chemotherapy phase is completed, proliferation is reduced and $\mathrm{Bcl}-2$ (an anti-apoptotic protein expressed in $80 \%$ of breast cancers) levels are decreased (Ellis et al., 1997). 
Extensive studies of the Bcl-2 family as well as p53, the tumour suppressor gene, has been done in breast cancer to describe its involvement in the apoptotic process (Parton et al., 2001). The activation of p53 can induce apoptosis and is considered to be one of the key mechanisms of action during DXR therapy. The Bcl-2 family include the molecules Bax, Bak and Bad, while Bcl-2, Bcl-xl and Mcl-1 are anti-apoptotic proteins (Parton et al., 2001). The principal executioners of cell death during apoptosis are proteolytic enzymes known as caspases, where eleven caspases have been associated with humans thus far (Parton et al., 2001). Caspases are inactive zymogens during synthesis and is activated through cleavage via an intracellular proteolytic cascade, providing them with the ability to induce apoptosis. Higher levels of apoptosis have been associated with increased cleavage of caspases 3, 6 and 8 (Shalini et al., 2015). Inhibitors of apoptosis (XIAP, NIAP cIAP1, cIAP2, and survivin) prevents the apoptotic pathway from continuing to the next phase by inhibiting the function of caspases, through direct binding to them (Hersey et al., 2003; Rathore et al., 2017). Apoptotic inhibitors can therefore potentially be used as predictive or prognostic biomarkers associated with cancer development. However, cancer cells are becoming more resistant to apoptotic cell death and higher dosages of chemotherapeutics are required to kill defective and mutated cells, but can also induce cell death in healthy tissues, which produces severe and unwanted side effects (Rathore et al., 2017).

\subsubsection{The p53 signalling pathway in apoptosis}

The p53 pathway responds to extrinsic and intrinsic stress signals that alter cellular homeostatic mechanisms responsible for monitoring chromosome segregation, DNA replication and cell division (Vogelstein et al., 2000). Once a stress signal occurs, the p53 protein is activated through post-translational modifications to induce cell cycle arrest (induces cellular growth arrest temporarily until damage is resolved) or apoptosis (Apella and Anderson, 2001). The p53 pathway activates proteins that can alter the cellular environment in response to cellular stressors (Figure 1.4). p53 mutations are often the most common mutational events associated with cancers (Harris \& Levine, 2005). 


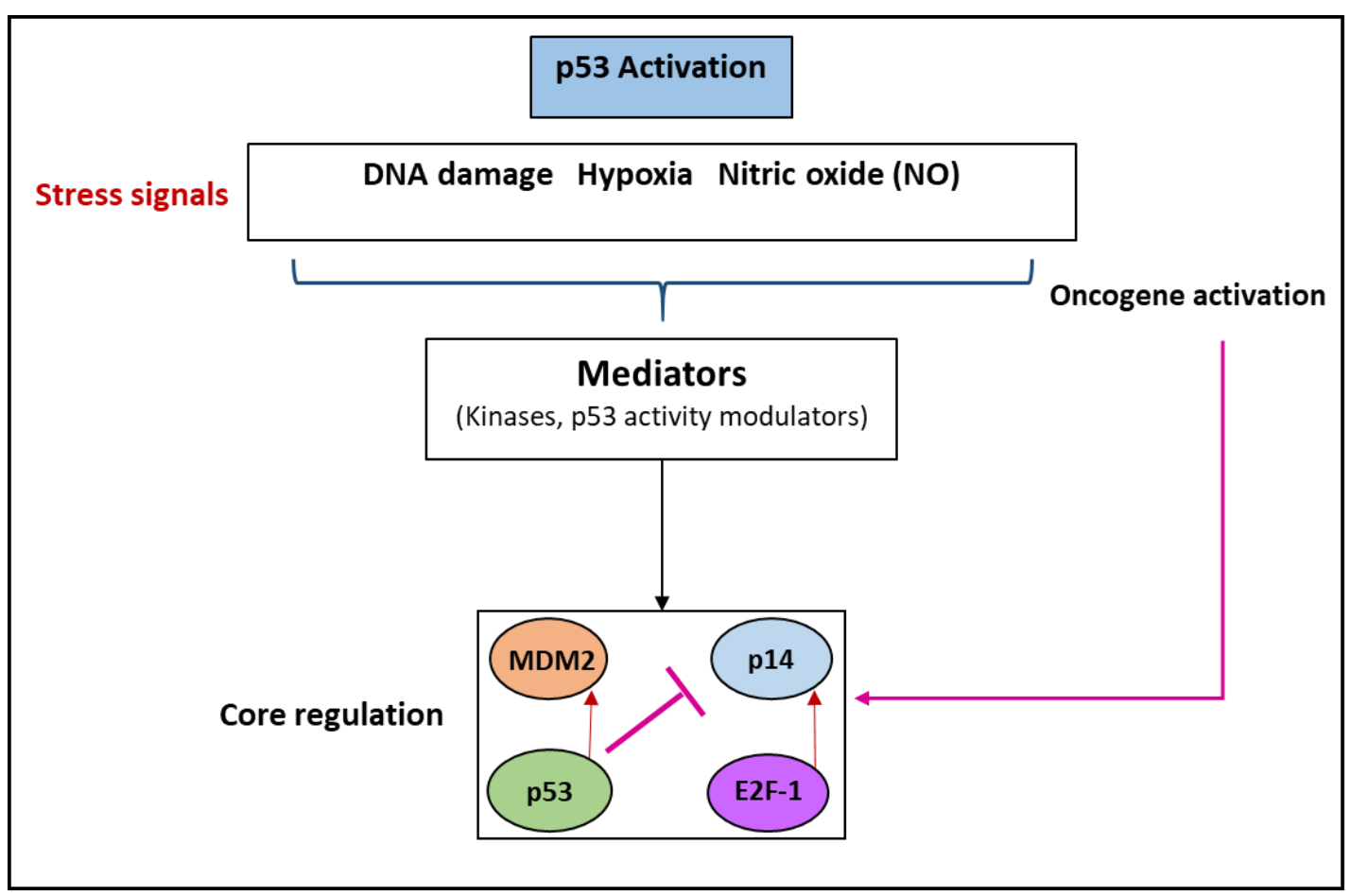

Figure 1.4: Different types of stress signals activate the p53 protein, which enhances the role of p53 as a tumour suppressor gene. DXR induces DNA damage in several cell types which can result in oncogene transformation. However, the activation of p53, a tumour suppressor protein, inhibits the function of E2F transcription factor 1 (E2F-1) resulting in cell cycle arrest (adapted from Harris and Levine, 2005).

DNA alterations or mutations associated with cancer often occur during base alkylation, ultraviolet (UV) or gamma irradiation and during the production of oxidative free radicals (Harris \& Levine, 2005). Fortunately, the cell is able to employ a different detection and repair mechanism, for each above-mentioned damaging agent. Proteins that are responsible for the detection and repair of DNA damage has specific enzymatic activities that indicates to the p53 protein that DNA damage has occurred (Broustas et al., 2014). The enzymatic activities induce post-translational modifications, which results in phosphorylation, methylation, acetylation or ubiquitination of the p53 protein (Harris \& Levine, 2005). Protein modifications modify the p53 pathway in two ways (Harris \& Levine, 2005). The first modification is the increase in the halflife of the protein in the cell, which is usually between six and twenty minutes, but changes to hours and results in a 3 to 10 -fold increase in p53 protein concentration within a cell. The second modification refers to the enhanced ability of p53 to promote transcription after binding to specific DNA sequences.

The p53 protein responds to many stress signals which can potentially disrupt cell duplication and lead to an increased cellular mutation rate or abnormality during cell division (Harris \& 
Levine, 2005). Therefore, p53 gene knockdown increases the susceptibility of an organism to develop cancer, even at a young age (Malkin et al., 1990). The limited ability of cells to divide once the cells are isolated from an organism and grown in culture is referred to as senescence. A senescent phenotype is therefore induced once cell division stops. Furthermore, p53 can also be activated from the mutational inactivation of certain tumour suppressor genes such as retinoblastoma (RB) (Sherr \& McCormick, 2002). The RB protein is therefore often found in cells in a complex with p53 and is associated with enhanced p53 activity and increased apoptotic activity (Xiao et al., 1995).

The p53 pathway therefore plays a dual role in preventing the onset of cancer in a cell population by firstly, inhibiting the cell cycle if the error rate is increased to prevent cancer cells from mutating and secondly by inducing apoptosis once mutations or severe damage occurs in the cell cycle.

\subsubsection{Apoptotic pathways}

As mentioned earlier, the p53 protein is part of two distinct apoptotic signalling pathways that result in the activation of caspases which mediate cell death (Vogelstein et al., 2000) (Figure 1.5). The extrinsic pathway is constituted of certain death receptors that is part of the tumour necrosis factor receptor (TNF-R) family and by forming a death inducing signalling complex (DISC), caspases can be activated to induce apoptosis (Locksley et al., 2001). The intrinsic pathway is activated once DNA damage occurs and is associated with mitochondrial depolarization and cytochrome $\mathrm{c}$ release into the cytoplasm and together with apoptotic protease-activating factor-1 (Apaf-1) and procaspase 9, cytochrome c forms an apoptosome to promote caspase activation (Zha et al., 1996; Wang et al., 2009).

\subsubsection{The extrinsic apoptotic pathway}

The p53 protein activates the extrinsic pathway by inducing genes that encode transmembrane proteins (Locksley et al., 2001). The TNF-R family is made up of cysteine-rich extracellular domains, referred to as 'death domains'. The death domain transmits the death signal at the cell surface receptor to the intracellular signalling pathway. The best identified ligands and death receptors include tumour necrosis factor (TNF)- $\alpha$ and tumour necrosis factor receptors-1 and -2 (TNFR1 and TNFR2); Fas ligand (FasL) and CD95/Fas receptor (FasR); TNF-related apoptosis inducing ligand (TRAIL) or Apo2Ligand (Apo2L) and death receptor-4 and -5 (DR4 and DR5) (Ashkenazi et al., 1998; Peter and Kramer, 1998; Suliman et al., 2001). Upon binding of their respective ligands, these death receptors trimerise and the 
resultant clustering of the associated cytoplasmic death domains results in the acquisition of adaptor molecules such as Fas-associated protein with death domain (FADD) that has a death effector domain (DED). The DED of the adaptor protein attaches to the DED of the inactive initiator caspase 8 , creating a complex called death inducing signalling complex (DISC) leading to the activation of an active initiator caspase. The activated initiator caspase in turn activates effector caspases (caspase 3/6/7) which leads to cleavage of substrates, ultimately inducing apoptosis (Kischkel et al., 1995).

\subsubsection{The intrinsic apoptotic pathway}

The intrinsic apoptotic signalling pathway has non-receptor mediated stimuli that produce intracellular signals (Elmore, 2007). These intracellular signals act directly on targets within a cell and is referred to as 'mitochondrial-initiated' events. Various damaging stimuli like toxins, hypoxia, free radicals and the absence of hormones and growth factors induce changes in the inner mitochondrial membrane, which results in an opening of the mitochondrial permeability transition pore (MPT) and loss of the mitochondrial transmembrane potential. Once mitochondria become depolarized, cytochrome $\mathrm{c}$ is released from the inner mitochondrial membrane into the cytosol. Cytochrome $c$ then binds to and activates Apaf-1 and procaspase 9 to form a heptameric protein complex termed the apoptosome (Chinnaiyan, 1999; Zha et al., 1996; Hill et al., 2004). Apaf-1 contains Deoxyadenosine triphosphate (dATP) as a cofactor and when cytochrome $C$ binds to Apaf-1, it induces hydrolysis of dATP to dADP, which is subsequently replaced by exogenous dATP. The active apoptosome recruits caspase 3 , which is then activated by caspase 9 to induce cell death.

Mitochondrial events during apoptosis is controlled and regulated by the Bcl-2 family of proteins (Cory \& Adams, 2002). Bcl-2 proteins maintains mitochondrial membrane permeability and can be pro- or anti-apoptotic. Pro-apoptotic family members are responsible for releasing cytochrome $\mathrm{c}$ from the mitochondria through pore formation (Kuwabara et al., 2002). Some cells do not die in response to the activation of the extrinsic pathway alone and requires another step where the intrinsic pathway is also activated. This step is induced by caspase 8 where it also targets the $\mathrm{BH} 3$-only protein Bid (BH3-interacting-domain death agonist) for cleavage to generate the activated fragment, t-Bid; t-Bid then directly activates proteins to induce mitochondrial outer membrane permeability (MOMP) to co-activate the intrinsic pathway (Li et al., 1998). Even though activation of these two apoptotic pathways is induced differently, the above mentioned suggests that cross-talk between the pathways facilitates the amplification of the extrinsic pathway via the intrinsic pathway, which is referred 
to as the 'mitochondrial amplification loop', to ensure that cell death is induced (Fulda \& Debatim, 2006).

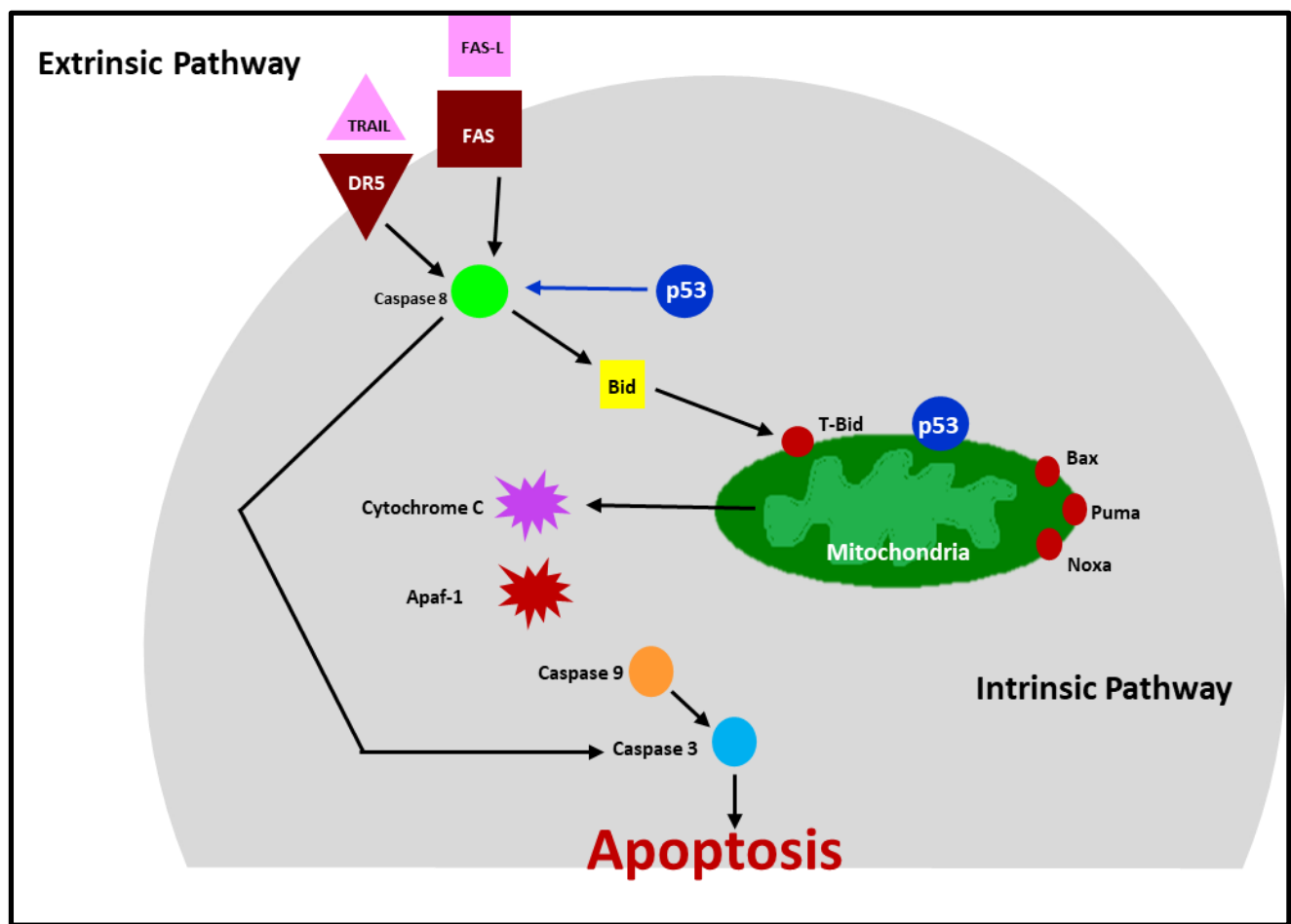

Figure 1.5: The intrinsic and extrinsic mitochondrial pathways of p53 induced apoptosis (adapted from Haupt et al., 2003).

Apoptosis significantly contributes to the tumour response after chemotherapy to ultimately prevent the cancer cells from growing (Kim et al., 2006). However, the amount of cell death from apoptosis after a lethal dosage administration of anti-cancer drugs does not always correspond with the observed tumour response. Clonogenic assays indicate that nonapoptotic forms of cell death occur as a late response and apoptosis induced cell death is an early response to DNA damage (Brown \& Wouters, 1999). Both apoptotic and other forms of cell death therefore contribute to the tumour response after chemotherapy because tumour cells have different drug sensitivities and are susceptible to more than one type of cell death. Several anti-cancer drugs therefore induce non-apoptotic forms of cell death, like autophagy, necrosis and extrinsic senescence (Ricci et al., 2004). 


\subsection{Senescence: a major contributor to therapy resistance}

Hayflick and Moorehead first introduced the concept of cellular senescence by describing the limited potential of human diploid cells after cell passaging to divide (Hayflick and Moorehead 1961; Lujambio, 2016). Multicellular organisms undergo cell division, which is important for cell survival and continued growth. Some tumour suppressor mechanisms act by diminishing the ability of damaged or mutant cells to develop into a tumour by eliminating the cell (apoptosis) or by permanently arresting the cell's proliferation (cellular senescence) (Davalos et al., 2010).

Cellular senescence is therefore defined as the proliferative arrest that occurs in normal cells after a limited number of cell divisions, but can also be referred to as a general biological program of terminal growth arrest (Roninson, 2003). Once senescence occurs, the cell cannot divide, not even through mitogen stimulation, but the cells remain metabolically active and show characteristic changes in morphology, including enlarged and flattened cell shapes and an increase in granularity. Three types of senescence have been identified: replicative, accelerated and oncogene-induced senescence (Gewirtz et al., 2008). The most common surrogate marker specific to senescent cells is senescence associated-B-galactosidase (SAB-gal) expression. X-gal can be used to stain for $S A-\beta-g a l(p H ~ 6.0)$ to reflect the increased lysosomal mass in senescent cells (Kurz et al., 2000).

During replicative senescence, cells undergo a restrictive number of divisions before they reach permanent growth arrest, which is determined from the shortening and structural alterations of telomeres, similar to the DNA damage response (Roninson, 2003). Accelerated senescence, characterised by the ability to rapidly induce a permanent state of growth arrest once cells are exposed to exogenous cytotoxic agents associated with DNA damage has the same biochemical and morphological features of replicative senescence (Campisi \& d'Adda di Fagagna, 2007; Gewirtz, 2008). Accelerated senescence can also be triggered by increased mitogenic signalling to induce rapid cell growth arrest, which does not involve telomere shortening. The p53 gene is commonly implicated in senescence once DNA damage occurs (Stansel et al., 2002). A downstream target of p53, p21 (a cyclin-dependent kinase inhibitor), is therefore also a critical component in the regulation of a senescent state (Zhang, 2007). However, the activation of p53 and p21 in senescent cells is only temporary and after growth arrest, the protein levels of p53 and p21 decline. When p21 decreases, p16 (a cyclin dependent kinase inhibitor), is upregulated which possibly indicates that p16 maintains growth arrest in senescent cells (Stein et al., 1999) (Figure 1.6). 


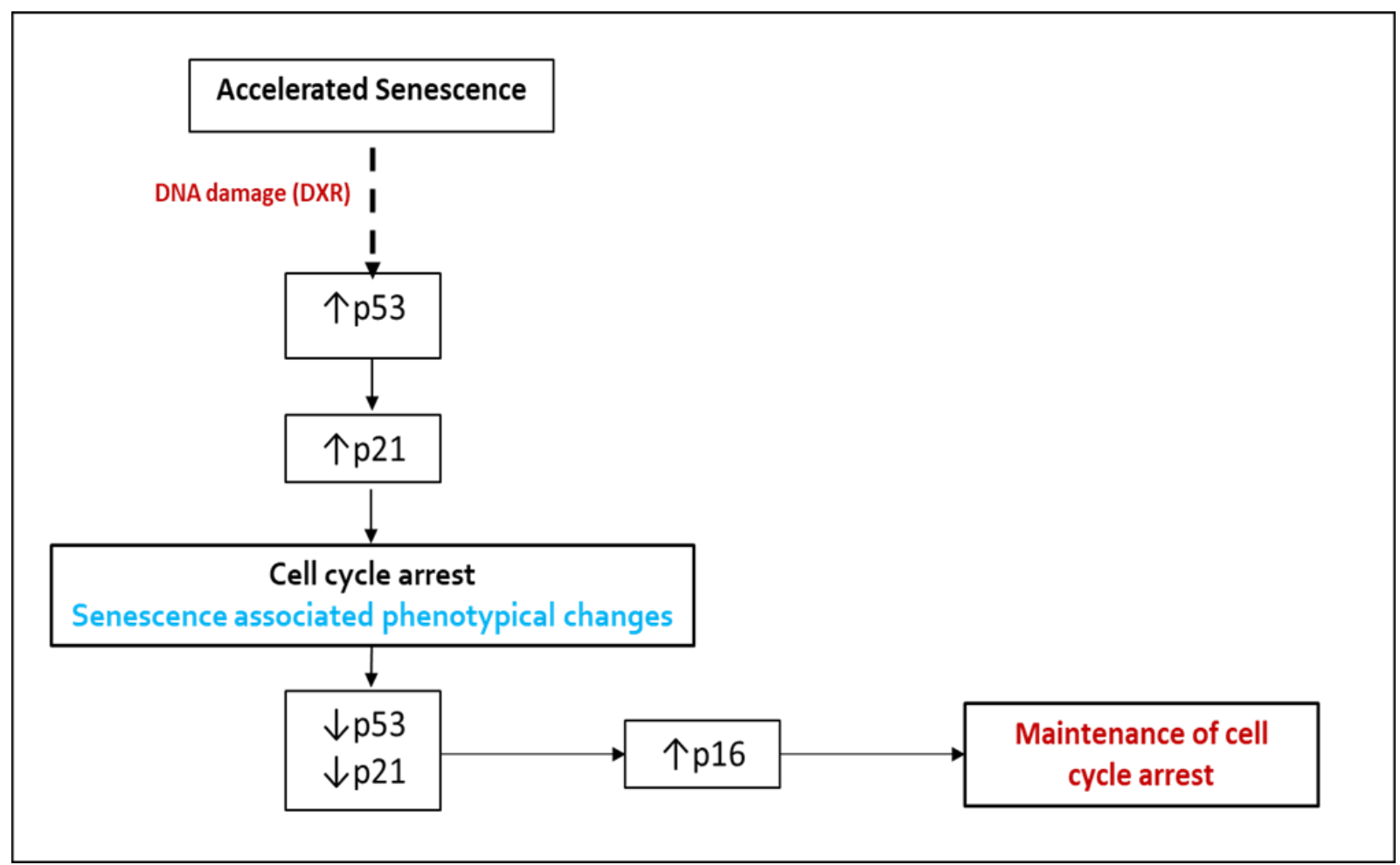

Figure 1.6: The p53 pathway controls cellular senescence via p21 activation (adapted from Roninson, 2003).

The inhibition of p21 or p53 expression reduces but does not block the senescence response in tumour cells after DXR treatment (Chang et al., 2002). This indicates that p53 and p21 are regulators of treatment-induced senescence in tumour cells, but they are not the only determinants thereof (Figure 1.6). Both forms of senescence (replicative and accelerated) are therefore essential anti-carcinogenic programmes found in normal cells (Roninson, 2003).

The anti-proliferative ability of the senescence response clearly highlights this process as a plausible tumour suppressor mechanism, based on its ability to stop the growth of cells that may become mutated or malignant (Narita et al., 2006; Gewirtz, 2008). Once a cell senses critical levels of damage or dysfunction, the senescence program is activated (Coppe et al., 2014). However, some studies also indicate that senescent cells often develop altered secretory activities that negatively influences the tumour microenvironment, which promotes tumourigenesis (Coppé et al., 2014). 


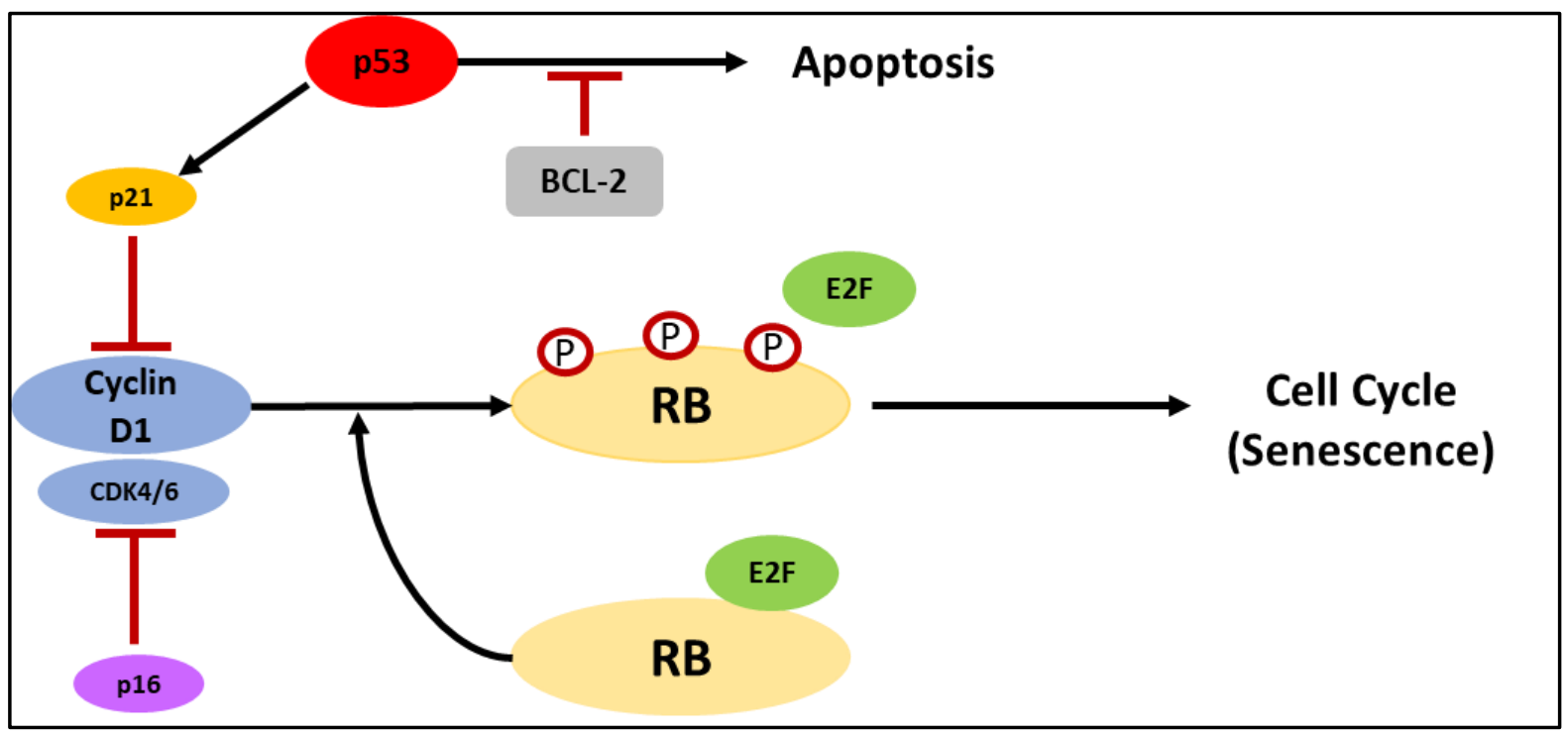

Figure 1.7: The p16/Retinoblastoma (RB) pathway is a major role player in cell cycle control (adapted from Jayasurya et al., 2005). The RB protein is activated by Cyclin D1 or CDK4/6, which results in its phosphorylation. $\mathrm{RB}$ is then inactivated and releases transcription factors that are needed for cell cycle progression. The p53 protein is activated in response to DNA damage, which can induce apoptosis. The p21 protein is transactivated by p53, which inhibits Cyclin D1 functioning.

In cell culture models, tumour development (in vitro) is promoted by the factors secreted from senescent cells by enhancing proliferation and invasiveness (Coppé et al., 2010). The phenomenon where senescent cells remain metabolically active but undergo changes in protein secretion and expression, is referred to as the senescence-associated secretory phenotype (SASP) and can occur in patients after they have received DNA damaging chemotherapy (Coppé et al., 2014). The SASP is constituted of many soluble and insoluble factors that affect neighbouring cells through the activation of cell surface receptors and signal transduction pathways. The SASP can be characterized into three components: signalling factors (chemokines, interleukins and growth factors), secreted proteases and secreted insoluble components (Coppé et al., 2010). Cytokines are known to have tumour promoting properties but is dependent on the relative concentration found in the surrounding cells and tissue (Knüpfer \& Preiss, 2007). Various cytokines are secreted by macrophages or lymphocytes and function to target nearby cells via a paracrine or autocrine manner (Duque \& Descoteaux, 2014). The immune systems of breast cancer patients are significantly impaired, which makes them more susceptible to metastasis.

The most important cytokine of the SASP is interleukin-6 (IL-6) (Ortiz-Montero, 2017). It is a pleiotropic pro-inflammatory cytokine which is associated with DNA damage and oncogenic stress-induced senescence in human and mouse melanocytes, monocytes, keratinocytes, 
fibroblasts and epithelial cells (Chang et al., 2002). In MCF-7 cells, IL-6 is considered to have a pro-survival function. In addition, fibroblasts in the TME secrete IL-6, which further stimulates the growth of tumour cells (Esquivel-Vela'zquez et al., 2015). This cytokine often allows cancer cells to evade apoptosis by inducing the activation of $\mathrm{Bcl}-2$ to increase resistance against DXR induced damage (Conze et al., 2001).

Interleukin-1 (IL-1) is also upregulated by senescent cells and is especially overexpressed in fibroblasts as well as in epithelial and endothelial cells after chemotherapy-induced senescence (Chang et al., 2002). An increase in the concentration of IL-1 is responsible for secondary responses that cause severe damage by enhancing the production and expression of many cytokines. These increases can then be linked with an increase in tumour cell proliferation and apoptosis inhibition (Apte et al., 2006). Cytokines are responsible for growth, cell signalling and differentiation of both tumour and stromal cells (Lewis et al., 2006). Cytokines secreted by the tumour cells alter the TME for optimal growth, while cytokines secreted by stromal cells further enhances the growth and malignancy of cancer cells. These cytokines (IL-6 and IL-1) target surrounding cells like fibroblasts to trigger nuclear factor Kappa $B$ (NFkB) activation which in turn allows tumour cells to enhance proliferation and block programmed cell death, by inhibiting the functions of caspases involved with apoptosis (Brasier et al., 2010). The NFkB pathway can be activated in most cell types and is therefore an important pro-inflammatory signalling pathway that functions to control DNA transcription, cell survival, immune response regulation and enhancing the expression of cytokines (Lawrence, 2009). Dysregulation of NFkB can therefore be linked to tumourigenesis, inflammation and improper immune development (Meffert et al., 2003). Senescent cells often overexpress insulin-like growth factor (IGF), which could possibly contribute to microenvironment alterations by allowing stromal cells to attract cytokines towards the TME to enhance an inflammatory state for optimal tumour cell proliferation (Mutgan, 2018). Senescent cells are able to create a permissive microenvironment that can allow cancer cells to grow. Tumour development can be promoted by various factors that are secreted from fibroblasts in the microenvironment and can be associated with various cell types found in breast (Krtolica et al., 2001), skin (Herbig et al., 2004) and prostate (Ben-Porath \& Weinberg, 2005) tissue.

The SASP dynamically develops over time creating a favourable environment for cancer cell proliferation (Coppé et al., 2008). In culture conditions, cells develop a full SASP five or more days after senescence has been induced while growth arrest usually occurs within 24 hours of damage, depending on the type of cell line. The loss of p53 due to genetic alterations results in a rapid SASP acquisition, which suggests that the development of the SASP is a specific 
program triggered through genotoxic stress. Data obtained by Coppé et al suggests that the SASP, specifically in senescent fibroblasts, is irreversible once it has formed (Coppé et al., 2008). However, p53 inactivation in senescent human fibroblasts with low levels of p16 can continue with cell proliferation, while retaining the SASP (Beausejour et al., 2003). High levels of p16 expression have been known to induce senescence where the RB tumour suppressor pathway is positively regulated by p16. p16 induces cell cycle arrest but does not seem to contribute to SASP development. Cells that are induced to senesce via ectopic $p 16$, express lower levels of SASP factors when compared to other senescent inducers (Coppé et al., 2008).

As previously mentioned, the tumour stroma is enriched with cytokines which attract and activate many cell types, including cancer associated fibroblasts. These cells closely interact with cancer cells to remodel the extracellular matrix and promote malignancy (Mishra et al., 2011). Similarly, senescent cells alter their tissue microenvironment towards a protumourigenic state by altering the function of neighbouring cells, which is often referred to as the "anti-cancer hypothesis" (Campisi, 1997; Campisi, 2005).

\subsection{The tumour microenvironment (TME) contributes to drug resistance}

For a long period of time, the main focus of cancer research has been on transformed tumour cells, which does not take the tumour microenvironment into consideration (Allinen et al., 2004). Most current cancer therapeutics only target the cancer cells, which are able to develop therapeutic resistance through inherent genomic instability as previously mentioned. However, recent in vitro and in vivo studies have demonstrated that surrounding stromal cells such as fibroblasts, myofibroblasts, myoepithelial cells and leukocytes influence the growth, differentiation and invasive behaviour of breast carcinomas (Hu et al., 2008).

Solid tumours obtained from breast epithelial tissue is supported by tumour stroma, which is defined as a non-malignant tumour compartment with various cell types as well as non-cellular components (Kucerova et al., 2013). Breast stroma is categorized by different types of cells like fibroblasts, adipocytes, endothelial cells, pericytes, immune cells and nerves (Wang et al., 2017). The interaction between stroma and epithelial cells is therefore critical for proper breast development because breast stroma has the ability to alter the proliferation, differentiation, invasion, metastasis and survival of cancer cells (Mao et al., 2013).

Anti-cancer agents like DXR have the ability to induce a senescent state in fibroblasts found in the TME (Yang et al., 2012). These senescent fibroblasts secrete a variety of bioactive 
molecules which promote tumour growth, metastasis, angiogenesis and drug resistance (Amornsupak et al., 2014). Activated fibroblasts, specifically in breast cancer, can be correlated with the level of aggressiveness of the disease because they have cancer promoting functions by guiding tumour cells through carcinogenesis via paracrine signalling, where proteins transmit "messages" in both directions that allow cross-talk between cancer cells and the stroma to occur (Aboussekhra, 2011). It is well established that normal cells in the surrounding TME have the ability to promote cancer cell growth once they are altered by anti-cancer drugs through the secretion of various factors (Li et al., 2007; Hu et al., 2008). It is suggested in a study that fibroblasts secrete collagen type 1, which decreases chemotherapeutic uptake into cancer cells and ultimately contributes to the mechanism of treatment resistance. These cells, now referred to as cancer associated fibroblasts (CAFs), provides tumour cells with energy sources such as ketones, lactate and fatty acids, by increasing the secretion of cytokines to reprogram energy metabolism to become more glycolytic (this is achieved by the inhibition of mitochondrial metabolism), which enhances the metabolic efficiency of cancer cells to promote tumour growth and survival (Peiris-pagès, Sotgia \& Lisanti, 2015).

Cancer cells are therefore surrounded by a complex microenvironment, which highlights the rationale that the TME should become a cancer research hallmark. The tumour microenvironment allows the cancer cells to survive by supplying the necessary nutrients and allows the cells to evade cell death and to metastasize (Chen et al., 2006; Degenhardt et al., 2006). Therefore, by fully elucidating the complex functioning and mechanisms of the TME, treatment strategies can be improved to avoid therapeutic resistance (Yang et al., 2015). As mentioned earlier, the physiological characteristics of the TME differ from those of normal tissue and is often described as hypoxic, nutrient deprived and inflammatory. These characteristics can induce autophagy through the activation of several pathways (Amaravadi et al., 2011). Autophagy is an essential self-eating process performed by cells to degrade intracellular components like organelles and aggregated proteins. The cross-talk between autophagy and the TME is therefore becoming an important area of clinical research to determine whether it contributes to chemotherapeutic resistance (Yang et al., 2015).

\subsection{The controversial role of autophagy in cancer development}

The autophagic process is defined as a catabolic process that degrades cytoplasmic components within a cell and thereby regulates homeostasis and cellular quality control by maintaining the turnover of organelles and proteins (Yang \& Klionsky, 2010). Cellular 
homeostasis is therefore highly dependent on the balance between the biosynthesis and catabolism of macromolecules (You et al., 2017). Two pathways responsible for protein degradation exist in eukaryotic cells: the ubiquitin-proteasome pathway and the lysosomal pathway (Myung et al., 2001). The ubiquitin pathway selectively degrades short-lived proteins, while the lysosomal pathway is responsible for the degradation and recycling of long-lived proteins and defective organelles (Rock et al., 1994). Autophagy related cell death (ARCD) is therefore classified as a type of regulated cell death that depends on the autophagic machinery or components thereof (Galluzi et al., 2018). The autophagy response is also activated upon cellular stress to mediate cytoprotective, rather that cytotoxic effects. Therefore, by blocking autophagy with pharmacological or genetic interventions, cell death will be accelerated rather than delayed which is seen in many pathological disorders including neurodegeneration and cancer.

The process of autophagy is involved in many biological processes, including cell death or survival, proliferation, senescence and the onset of carcinogenesis (Levine \& Klionsky, 2004). However, the role of autophagy in cancer remains controversial (Kimchi et al., 2004; Kageyama \& Komatsu, 2012) (Figure 1.8). The process allows cancer cells to recycle and restore cytoplasmic components for survival, but excessive levels of autophagy induces type II programmed cell death, which is considered to be a non-apoptotic form of cell death and can be induced by cytotoxic stimuli, such as DXR (Cagnol and Chambard, 2010; Bernard and Klionsky, 2013). Autophagy regulation can therefore be increased or inhibited by anti-cancer agents where an increase in autophagy could promote cancer cell survival and an excessive increase could induce cell death, depending on the tumour type and complexity of the tumour (Gewirtz et al., 2016).

The autophagy process is initiated by the formation of a crescent shaped double membrane, a phagophore, which is closely associated with microtubule-associated protein 1A/1B-light chain 3 (LC3) (Kirisako et al., 1999). LC3 is a soluble protein found in cultured cells as well as mammalian tissues, with a $17 \mathrm{kDa}$ molecular mass (Tanida et al., 2008). During the onset of autophagy, LC3 in its cytosolic form (LC3-I) is conjugated to phosphatidylethanolamine to form LC3-phosphatidylethanolamine conjugate (LC3-II). LC3-II in the autolysosomal lumen is also degraded with the engulfed content and is therefore closely associated with lysosomal turnover during autophagic activity. 
The phagophore then engulfs ubiquitinated substrates to form an autophagosome, which fuses with a lysosome to form an autolysosome. The autolysosome is characterized by an internal acidic and hydrolytic environment to degrade the content within the lumen of the autolysosome (Tanida et al., 2008).

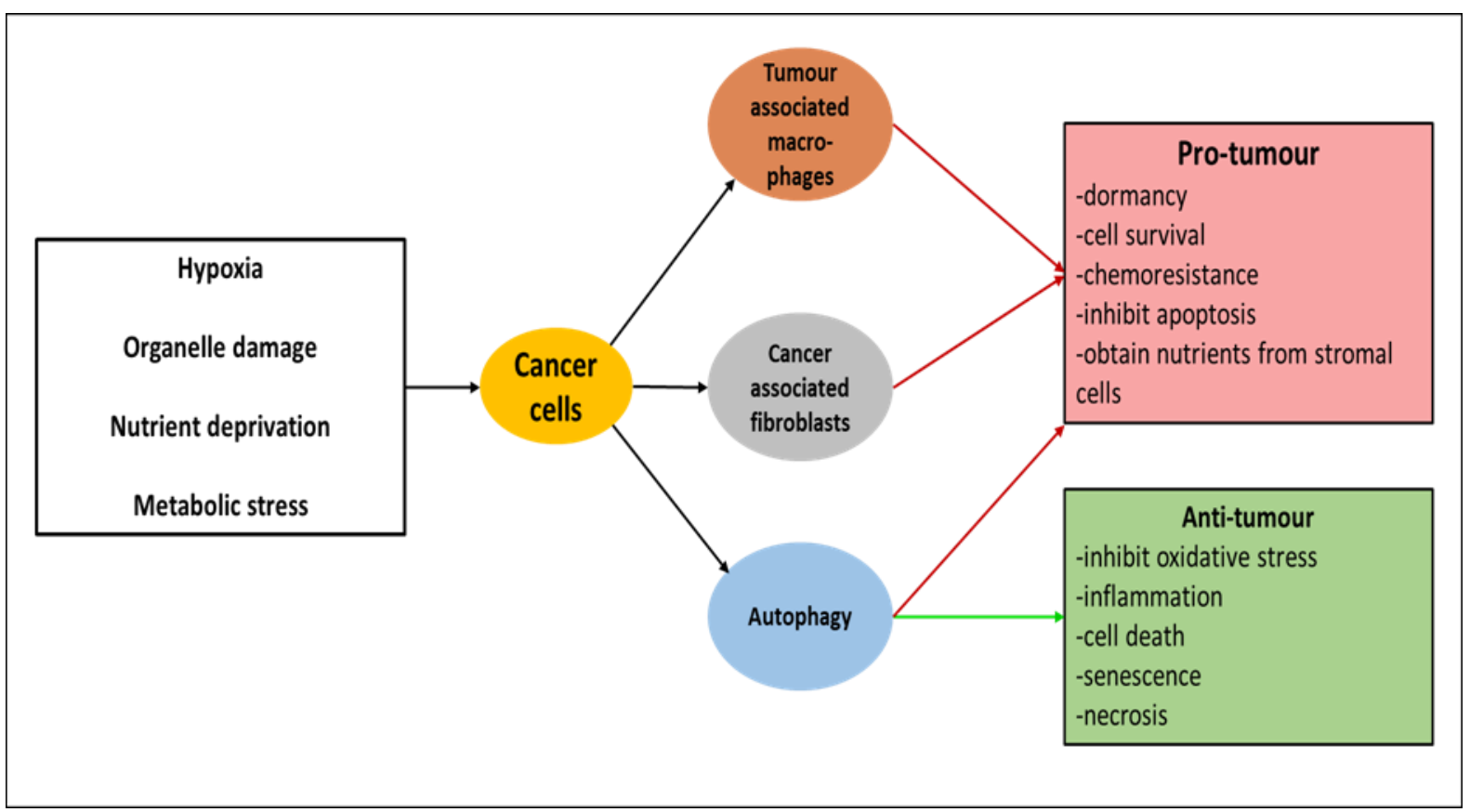

Figure 1.8: The controversial role of autophagy in cancer. Autophagy can promote tumour cell survival but can also participate in cell death (adapted from Ngabire and Kim, 2017).

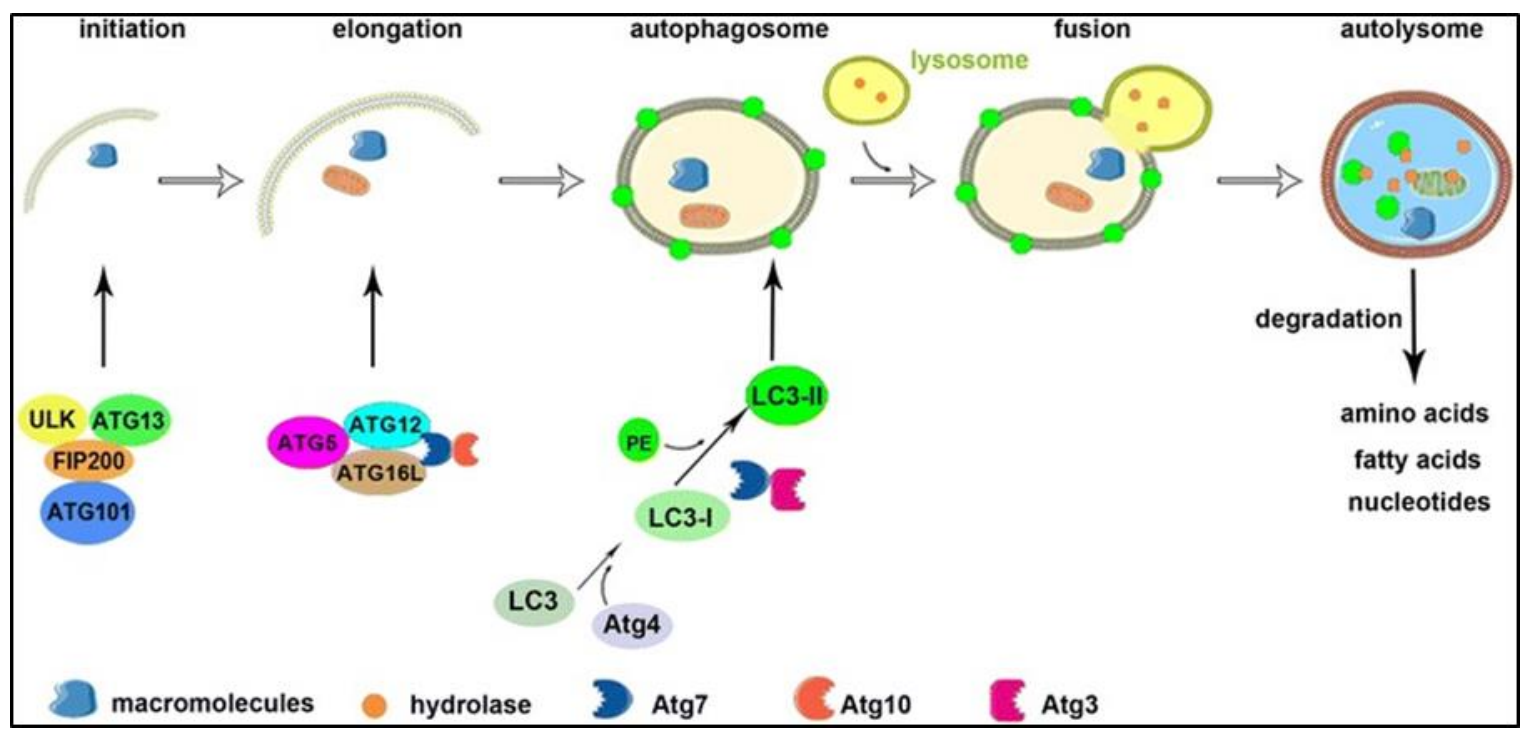

Figure 1.9: The autophagy process. The autophagy process is categorized into 5 stages: initiation, elongation, autophagosome formation, fusion and autolysosome formation (from Yang et al., 2015). 
Basal levels of autophagy are regulated by the mammalian target of Rapamycin complex 1 (mTORC1), which is a downstream effector of the Phosphoinositide 3-kinase (PI3K) pathway and the phosphorylation of autophagy related (ATG) gene 13 (ATG13) (He \& Klionsky, 2010; Frankel \& Lund, 2018). The mTORC1 complex monitors the nutritional state of the cell and maintains homeostasis by regulating the ratio between anabolic and catabolic processes.

Currently, 40 autophagy related genes (ATG) that partake in autophagy or autophagy related processes have been identified (Klionsky et al., 2014). The first identified autophagy adaptor, also referred to as a cargo receptor, was p62, which is considered to be a pro-oncogenic regulator (Moscat et al., 2009). p62 was first discovered in mammals as a selected autophagy adaptor and is a multifunctioning protein that is constituted of an N-terminal Phox-BEM1 domain (PB1), a nuclear localization signal (NLS), an LC3 interacting region (LIR), an export motif and a C-terminal ubiquitin associated domain (UBA) (Wang et al., 2013; Lin et al., 2013; Liu et al., 2016). The function of p62 is to transport ubiquitinated cargoes to the UBA or LIR domain for autophagic degradation (Myeku et al., 2011). The activation of autophagy will therefore decrease the expression of p62 because the accumulation of p62 can have detrimental effects (Komatsu et al., 2007). Ultimately, p62 will bind LC3-II to promote autophagic degradation. It also mediates the aggregation of damaged mitochondria, by allowing LC3-II to bind, where after damaged mitochondria can be transported to the autophagosome for degradation. High doses of DXR could therefore potentially increase p62 expression to deliver the damaged mitochondria to the autophagosome to be degraded, which will inhibit the cancer cells to continue growing (Koleini et al., 2017).

Autophagy promotes cancer cell survival by protecting the cells from oxidative stress, ROS, starvation and hypoxia which also allow the cancer cells to develop chemoresistance (Fiaschi \& Chiarugi, 2012; Suman et al., 2014) (Figure 1.10). However, the functions of autophagy are highly dependent on the cell type, the TME and the duration of the treatment. In some patients, autophagy is upregulated in tumours once the treatment period is initiated, which allows the tumour cells to protect themselves against therapy-induced apoptosis (Brech et al., 2009). 


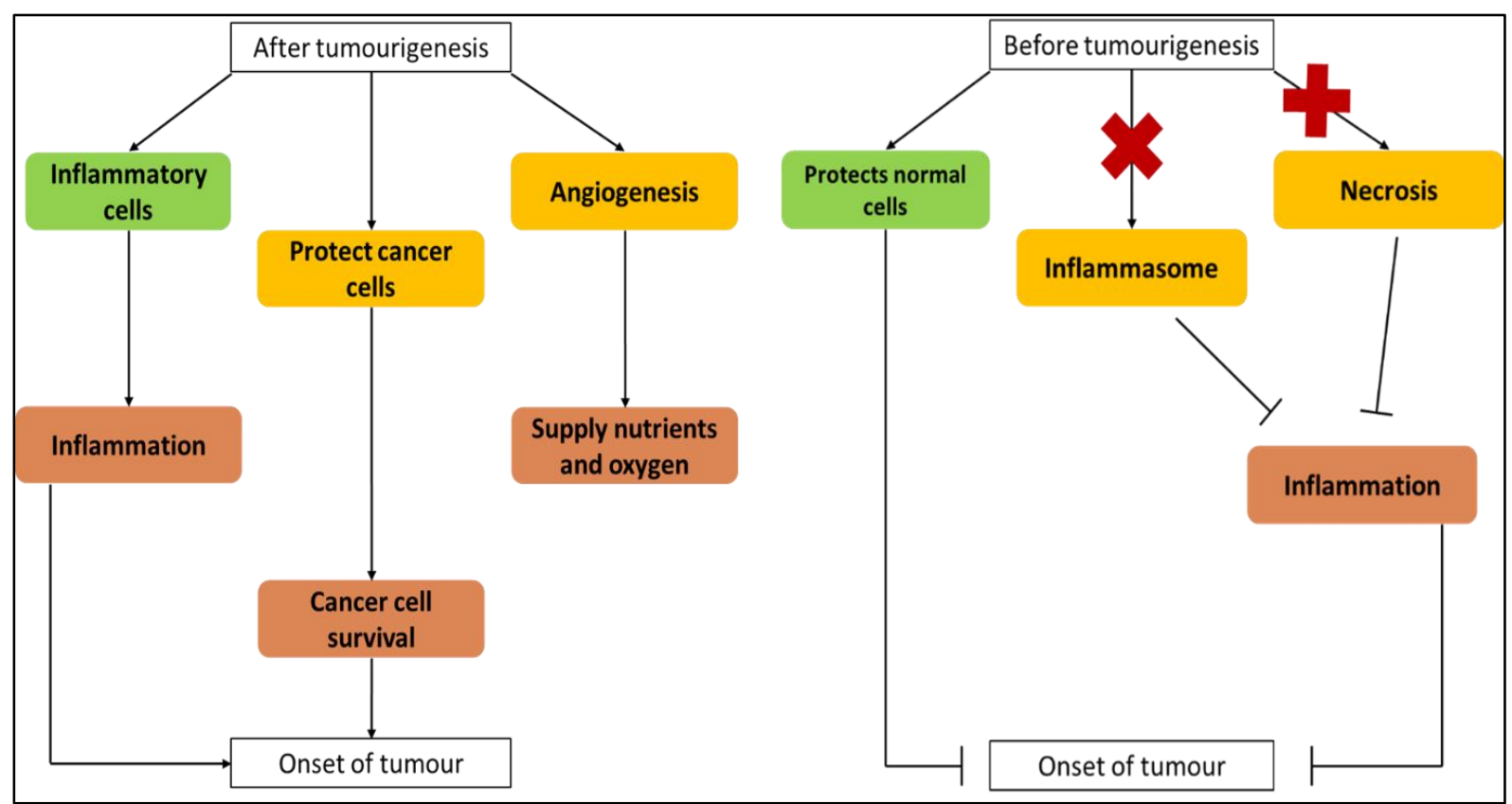

Figure 1.10: Autophagy promotes cancer cell survival by protecting the cells from oxidative stress, ROS, starvation and hypoxia which allows the cancer cells to develop chemoresistance and continue growing (adapted from Yang et al., 2015).

However, some studies indicate the opposite effect of autophagy and conclude that autophagy increases apoptotic and caspase-independent cell death in cancer cells (Colell et al., 2007). In most cases, autophagy sustains cancer cell viability once the apoptotic process becomes defective (Lum et al., 2005; Karantza-Wadsworth et al., 2007). Defective apoptosis allows the cancer cells to tolerate long term metabolic stress (White et al., 2007). Cells with PI3K mutations or Akt (protein kinase B) activation (characteristic during tumour development) is therefore dependent on autophagy for energy homeostasis and survival. Proliferating tumour cells are highly disadvantaged once autophagy is inhibited because compensatory mechanisms must be implemented for cancer cell survival (Mah et al., 2012).

It is well established that autophagy is regulated by many signalling pathways, including the PI3K-mTOR signalling pathway, which is a key regulator of the autophagic process (Klionsky, 2005; Esclatine et al., 2009; He and Klionsky, 2009). As mentioned previously, the PI3K pathway is responsible for many cellular regulatory processes including cell differentiation, cell survival and proliferation (Engelman et al., 2006; Liu et al., 2009). The PI3K pathway is activated in most human cancers, but multiple components of the PI3K pathway are easily mutated or modified, which disrupts autophagy regulation and can potentially result in the onset of tumourigenesis (Liu et al., 2009). The PI3K pathway is therefore an ideal target for cancer therapy (Massacesi et al., 2016). The first PI3K pathway-targeted agents were 
rapamycin analogues, which allosterically inhibit mTORC1 (Dienstmann et al., 2014). Several PI3K-inhibitors are currently under clinical investigation, including pan-PI3K inhibitors that target all four isoforms of PI3K, as well as isoform selective inhibitors like copanlisib, pictilisib and taselisib (Dienstmann et al., 2014). However, by inhibiting the PI3K pathway, high mobility group box 1 (HMGB1)-induced autophagy is also significantly inhibited (Sun et al., 2015).

\subsection{High mobility group box 1 promotes an inflammatory state in cancer development}

HMGB1 was discovered 40 years ago in calf thymus and was named for its fastelectrophoretic mobility in polyacrylamide gels (Zhang et al., 2012). Currently, it is referred to as a non-histone chromosomal protein that is ubiquitously found in all vertebrate nuclei that binds double-stranded DNA, independent of sequence specificity (Sun et al., 2015).

The HMGB1 protein is found in most cell types and is normally confined in the nucleus where it regulates protein transcription, by functioning as a nuclear cofactor (Sun et al., 2015). HMGB1 is passively released from necrotic cells and is also actively secreted by inflammatory cells, which binds to several receptors like the receptor for advanced glycation end products (RAGE) and Toll-like receptors 2 and 4 (TLR2 and TLR4) (Lotze et al., 2005; Dong et al., 2007). HMGB1 is therefore implicated in many disease states including sepsis, neurodegeneration and cancer (Wang et al., 2004; Ellerman et al., 2007; Sparvero et al., 2009). Similarly, a few studies have also indicated that the levels of HMGB1 and RAGE are overexpressed in various human neoplasms (prostate, pancreatic and colorectal cancer), when compared to the surrounding normal epithelium (Pardo et al., 2006; Volp et al., 2006). The overexpression of HMGB1 is associated with the hallmarks of cancer: unlimited replicative capacity, angiogenesis, resistance to growth inhibitors, evasion of apoptosis, inflammation and tissue invasion and metastasis (Hanahan \& Weinberg, 2000). HMGB1 is therefore the prototypic Damage Associated Molecular Pattern (DAMP) associated with an acute and chronic inflammatory response and wound repair (Land et al., 1994; Matzinger, 2004). DAMPs are endogenous molecules that are released into the extracellular space when tissue damage or cellular stress is present (Alvarez \& Vasquez, 2017). Pattern recognition receptors (PRR) identify these molecules to induce inflammation and an immune response, without an infection present. HMGB1 overexpression is therefore strongly correlated with tumour invasiveness in cancer patients. A few studies have suggested that the growth of breast cancer cells in vitro is promoted by HMGB1, however the exact role of HMGB1 in breast cancer patients is not yet fully elucidated (Jiao et al., 2007; Chalmers et al., 2013). 
As previously mentioned, cancer cell death can be induced by many factors, including chemotherapy (anti-cancer treatments). Tumour cells treated with chemotherapy release HMGB1 to induce inflammation, by stimulating the release of TNF- $\alpha$ and IL-1 $\beta$ (Bustin, 2002; Dong et al., 2007). When HMGB1 is present in the extracellular medium, it indicates that the cells have become stressed or have died. These cells are then able to alert other cells in the nearby environment to avoid the immediate danger (Bianchi, 2009). Furthermore, HMGB1 is also suggested to play a major role in apoptosis, depending on the cell type and stress inducer (Tang et al., 2010). HMGB1 protects mammalian cells against the damaging effects of death stimuli, like radiation by avoiding CD95 activation and caspase 8- and Bax-induced apoptosis (Brezniceanu et al., 2003). To establish HMGB1's role in treatment resistance, Lang et al indicated that HMGB1 knockdown in MEFs causes hypersensitivity to UV radiation, which supports the ability of HMGB1 to protect cells against cell death or apoptosis (Lang et al., 2008).

Furthermore, Sun et al investigated HMGB1 expression in both tissue and serum in breast cancer. HMGB1 expression was greater in breast cancer tissue, when compared to the surrounding normal tissue and thereby supports the role of HMGB1 in tumorigenesis (Sun et al., 2015). The authors also provided possible mechanisms that allows HMGB1 to induce tumour development:

1: HMGB1 overexpression causes certain gene mutations that may lead to certain tumour phenotypes.

2: High levels of HMGB1 promotes tumour growth by preventing apoptosis induction (Muller et al., 2004).

Cancer patients often develop resistance towards chemotherapeutic drugs after a few years, which allows the cancer to reoccur (Dalton, 1997). As mentioned earlier, DXR induces cell death or growth arrest, but DXR resistance often occurs in advanced tumours, which result in a poor prognosis (Kruh \& Goldstein, 1993). The involvement of autophagy in DXR resistance is therefore being extensively studied and the inhibition or activation of autophagy can therefore partly overcome DXR resistance in many cancer types, which suggests that autophagy modulation can be a promising strategy to overcome DXR resistance (Chen et al., 2017). 


\subsection{Autophagy contributes to DXR resistance in cancer}

Autophagy causes cell death or will allow cancer cells to survive, depending on the treatment duration and the severity thereof (Aydinlik et al., 2017). Several studies suggest that during nutrient deficiency, many therapeutics such as tamoxifen (a chemotherapeutic agent) induces autophagy as a pro-survival mechanism to ensure that cancer cells continue growing (Zarzynska et al., 2014).

However, in some studies, autophagy is considered to be a tumour suppressive mechanism and is therefore characterised as being cytotoxic towards cancer cells. DXR is most commonly used to treat triple negative breast cancer (TNBC) and studies indicate that once cytotoxic autophagy is induced, the ability of DXR to kill cancer cells is diminished (Tani et al., 2015). Many clinically approved anti-cancer agents, like DXR, upregulate autophagy in cancer cells in vitro and by inhibiting autophagosome formation or autophagy signalling pathways (PI3K and mTOR), cancer cell death can be promoted (Kondo et al., 2005).

DXR is therefore commonly used as an anti-cancer drug worldwide to kill cancer cells through apoptosis by inducing DNA damage (Lee et al., 2002). Moderate doses of DXR can also induce a senescence phenotype in cells, especially in normal fibroblasts (Roninson, 2003). In 2009, Young et al reported that autophagy-related genes are upregulated during oncogene induced senescence and that by inhibiting autophagy, the onset of a senescence phenotype can be delayed (Young et al., 2009).

\subsection{The role of apoptosis and senescence in autophagy}

Cross talk between autophagy and apoptosis is well-established, however, there is little evidence to support the potential of a direct relationship between autophagy and senescence (Gewirtz, 2007; Thorburn 2008). When autophagy is inhibited, cancer cells undergo apoptosis, but dysregulated apoptosis is commonly present in human cancers and cancer cells die by other cell-death mechanisms (Degenhardt et al., 2006). It is therefore suggested that autophagy is another form of cell death in apoptosis resistant cancer cells, but the exact mechanisms are not known (Maiuri et al., 2007). Cross talk between autophagy and apoptosis exist because the two pathways share the same mediators for upstream or downstream signalling. Studies therefore suggest that autophagy is linked with the extrinsic pathway, 
mediated by p62/Sequestome 1 (SQSTM 1) when it binds and activates caspase 8 to enhance TRAIL-mediated apoptosis (Jin et al., 2009).

The link between senescence and autophagy has not fully been elucidated, but senescence and autophagy have many similar characteristics and processes that have the same outcome for cells. Both senescence and autophagy induce responses that can either be cytoprotective or cytotoxic after chemotherapy or radiation (Gewirtz, 2013). The study of Young et al was the first to identify a possible relationship between autophagy and senescence in fibroblasts (Young et al., 2009). They concluded that autophagy can delay the oncogene-induced senescence response. To further support the above-mentioned findings, David Gewirtz indicated that autophagy and senescence is regulated by overlapping signalling pathways such as ROS production, induction of p53 and p21 and dephosphorylation of Retinoblastoma (RB) protein (Gewirtz, 2007). Other groups have also reported that autophagy promotes senescence by overexpressing CDK inhibitors (p16 and p21) in both fibroblasts and breast cancer cells (Capparelli et al., 2012). However, it is also known that autophagy supresses senescence to maintain cellular homeostasis, especially during excessive oxidative stress levels or during mitochondrial dysfunction, where uncoupling mitochondria reduces superoxide generation to limit the senescence response (Kwon et al., 2017).

Furthermore, a senescent state is also associated with tumour dormancy (Gewirtz, 2007). Tumour dormancy generally occurs at the primary tumour site, where cells were not successfully removed during radiation or surgery. These cancer cells are also able to remain dormant in brain or bone tissue once the cells have metastasized (Gewirtz et al., 2009). Once these tumour cells enter an active dividing state, the cells will continue to grow, which results in cancer recurrence.

Autophagy induces an effective immune response in cancer cells exposed to chemotherapeutics and similarly during senescence, the cell induces a prolonged state of growth arrest to avoid stress induced toxicity (Michaud et al., 2011). Both processes are therefore able to evade cellular death. In 2009, Yang et al reported that autophagy related genes are upregulated in oncogene-induced senescence and that autophagy inhibition delays the senescent phenotype, which indicates that these cells reflect efforts to try and evade the direct cell death response (Yang et al., 2009). In addition to the above-mentioned study, Goehe et al indicated that senescence is temporarily blocked during pharmacological 
inhibition of autophagy (Goehe et al., 2012). It seems that autophagy accelerates the senescence response by providing energy sources, but the authors concluded that senescence can occur independently from autophagy.

Although a direct link between senescence and autophagy has not been established, some studies have reported that both processes signal the immune system to eliminate a growing tumour because both are associated with an inflammatory response and the production of various cytokines (Ewald et al., 2010; Michaud et al., 2011). In addition to the above mentioned, it is also suggested that autophagy and senescence are regulated through overlappingexc signalling pathways such as ROS production, the induction of p21 and p53 and dephosphorylation of RB and the overexpression of several CDK inhibitors (p16, p19 and p21) has shown to activate the autophagic pathway in several cell types (Gewirtz, 2007). In some cases, autophagy suppresses senescence to maintain cellular homeostasis especially during excessive oxidative stress levels or during mitochondrial dysfunction, but remains to be further elucidated (Young et al., 2009; Kwon et al., 2017). Apoptosis, senescence and autophagy therefore interconnect in cancer development and growth and could alter the response of tumours to anti-cancer therapy (Powlaska et al., 2018).

Normal cells, like fibroblasts, in the surrounding TME are also affected by DXR and several studies have shown that once fibroblasts adopt a senescence phenotype, apoptosis induction in cancer cells is limited (Tao et al., 2017; Wang et al., 2017). Cancer cells coevolve with the TME, therefore the ability of autophagy to modulate interactions between cells in the TME is a potential factor in determining whether autophagy inhibition can be effective in cancer treatment (Levy et al., 2017).

\subsection{The role of autophagy in the tumour microenvironment (TME)}

As mentioned previously, the TME contributes to cancer cell survival, metastasis and invasion (Yang et al., 2015). The TME and normal tissues differ physiologically, where the TME has been described as nutrient deprived, hypoxic and inflammatory (Amaravadi et al., 2011). Studies have shown that between $50-60 \%$ of tumours survive in hypoxic conditions and one possible mechanism that contributes to their ability to survive is by increasing the activation of the autophagic pathway (Vaupel et al., 2007). 
Tumour progression in the TME is greatly associated with the onset of inflammation (Coussens et al., 2002). Chronic inflammation favourably promotes tumorigenesis and enhances the ability of cancer cells to metastasize (Ngabire \& Kim, 2017). Highly plastic leukocytes, referred to as macrophages, enter various tissues under inflammatory conditions and adopt different phenotypes and functions depending on the cues that they receive from the environment (Ariel et al., 2012). Macrophages in the TME are recruited from monocytes found in blood vessels and are polarized into tumour associated macrophages (TAMs). The two known phenotypes for TAMs are M1 macrophages that are pro-inflammatory and M2 macrophages, which are anti-inflammatory (Franklin et al., 2014). Inflammatory cytokines such as IL-6, IL-10 and TGF- $\beta$ are released by TAMs and contributes to the development of cancer by limiting apoptosis in the TME. Cytokines therefore play a major role in the development of chronic inflammation to generate a pro-tumour response (Ngabire \& Kim, 2017). Chronic inflammation is generally also associated with an increase in oxidative stress which activates NF-kB to stimulate the onset of autophagy to allow cancer cell growth (Joven et al., 2014). Once autophagy is activated, other cell death pathways like PARP-mediated cell death induced by DXR becomes impaired, which will allow the cancer cells to continue proliferating (Degterev et al., 2008).

Tumour resistance to chemotherapy is therefore often associated with autophagy due to the enhanced ability of cancer cells to evade cell death and to rapidly proliferate (Yang et al., 2015). By inhibiting autophagy, chemosensitivity could possibly be enhanced, therefore elucidating the impression that a combination of cytotoxic drugs and an autophagy inhibitor could be a possible treatment therapy for breast cancer patients (Yang et al., 2011).

\subsection{Inhibition of autophagy can enhance cancer cell death}

Autophagy functions to control homeostasis in all living organisms and dysregulation of this pathway could therefore support and enhance tumour development (White \& DiPoala, 2009; Katayama et al., 2007). Autophagy allows oncogenic transformation to occur by limiting the occurrence of chronic inflammation, which is responsible for the release of pro-inflammatory HMGB1 (stimulates NFKB-induced transcription). Studies therefore suggest that autophagy increases the ability of cells to tolerate stress, which promotes tumour survival (Guo et al., 2011). Inactivation of autophagy (by activating the PI3K pathway and mTOR) prevents the cells from surviving during metabolic stress (Tanida et al., 2008). Therefore, in cells with a 
defect in apoptosis, this could ultimately result in necrosis mediated cell death (Elmore, 2007). However, the specific trigger that activates the onset of necrosis is unknown.

Many authors have concluded that autophagy is required to maintain protein and organelle quality control and basal levels of autophagy are present in normal physiological conditions (Das et al., 2012; Mathew et al., 2007). Cancer cells with apoptosis defects could result in DNA and genome damage, which contributes to the tumour promoting properties of autophagy (Tanida et al., 2008). Once genome damage occurs, cellular functions that maintain genome integrity (DNA replication and repair) is severely damaged.

The ability to inhibit autophagy therefore has great potential as a target for cancer therapy, once apoptosis resistant cancer cells become sensitized to metabolic stress (Maycotte \& Thorburn, 2011). Conventional therapies such as surgery and chemotherapy can potentially disrupt tumour architecture and vascularization and remaining tumour cells could then become susceptible to metabolic stress and autophagic inhibitory therapy, which could prevent tumour regrowth and metastasis (Hayat, 2013). Pharmacological inhibitors of autophagy like Bafilomycin A1 and Chloroquine are used in cells and tissues to determine whether autophagy has protective or damaging effects in normal and pathological states because of its controversial role in cancer development (Maycotte \& Thorburn, 2011). Recently, a clinical trial in America aimed to determine the effects of autophagy inhibition with Chloroquine in DCIS and whether it will reduce the ability of the cancer cells to survive. Participants received either chloroquine standard dose (500 mg/week) or chloroquine low dose (250 mg/week) for 1 month prior to surgical removal of the tumour (Martinez-Outschoorn et al., 2010).

Bafilomycin was first isolated from the mycelium of Streptomyces and was defined as a macrolide antibiotic able to prevent or inhibit the growth of fungi, yeast and gram-negative bacteria (Dodsen et al., 2013). The ability of Bafilomycin to inhibit vacuolar ATPase (VATPase), the enzyme responsible for vesicular acidification and $\mathrm{pH}$ control in the lysosome, allowed for its functional classification (Lee et al., 2012). During autophagy, lysosomes and autophagosomes fuse together and Bafilomycin can prevent this fusion by inhibiting $\mathrm{V}$ ATPase. Therefore, inhibition of the fusion process will ultimately prevent the autophagic flux from occurring (Bowman et al., 1988; Yosimori et al., 1991). 
The ability of cancer cells to develop resistance against chemotherapy treatment is still however a major concern (Housman et al., 2014). Chemotherapy kills cancer cells by effectively stopping the cell division process. Anti-cancer drugs damage the RNA or DNA that is responsible for the cancer cell being able to copy itself during cell division (Lodish et al., 2000). If the cancer cells are unable to divide, they die. However, the proliferation of a tumour cell line can be enhanced in vitro by conditioned media (CM) obtained from fibroblasts, which possibly indicates that soluble factors are present in the $\mathrm{CM}$, which will allow the cancer cells to evade apoptosis (Pistone et al., 2013). Furthermore, senescent cells within in the tumour microenvironment communicate with neighbouring cells by secreting factors that constitute the SASP (cytokines, chemokines, and growth factors) (Lujambio et al., 2016). Senescent fibroblasts therefore have the ability to enhance the proliferation of cancer cells by secreting these molecules. A limitation of current cancer therapies like chemotherapy and radiation is that many cancers develop resistance to apoptosis and therapies that target alternative pathways should therefore be considered (Fung et al., 2008; Maycotte \& Thorburn, 2011). However, the role of autophagy in tumourigenesis still remains controversial. Increased autophagy induces autophagic cell death in apoptosis defective cells, but inhibiting autophagy could also prevent autophagy mediated resistance against chemotherapy (Shimizu et al., 2004; Akar et al., 2008). Therefore, for the future development of effective cancer therapy with minimal side effects, it is important to determine whether autophagy is a protective mechanism that decreases cancer cell death or if it promotes apoptotic cancer cell death. 


\subsection{Hypothesis}

Based on the current literature, we hypothesize that fibroblasts can secrete paracrine factors that affects breast cancer growth and DXR resistance, however, these effects might differ when senescence and apoptosis is induced in the fibroblasts.

\subsection{Aim}

The aim of this study is to assess the effects of conditioned media harvested from senescent and apoptotic fibroblasts on breast cancer cells to determine whether senescent fibroblasts and autophagy contribute to the ability of breast cancer cells to evade apoptotic cell death.

\subsection{Objectives:}

- To treat mouse embryonic fibroblasts (MEFs) with DXR and determine a concentration that induces apoptosis and senescence in these cells by performing cell viability and senescence-associated $\beta$-galactosidase assays as well as assessing the protein expression of markers for each process with western blots.

- To generate conditioned media from MEFs treated with optimised DXR concentrations.

- To determine the sensitivity of mouse breast cancer cells (E0771 cells) to DXR when exposed to conditioned media from apoptotic and senescent MEFs.

- To determine the effect of conditioned media treatment and autophagy inhibition in E0771 cells. 


\section{Chapter 2: Methods and Materials}

\section{Study design}

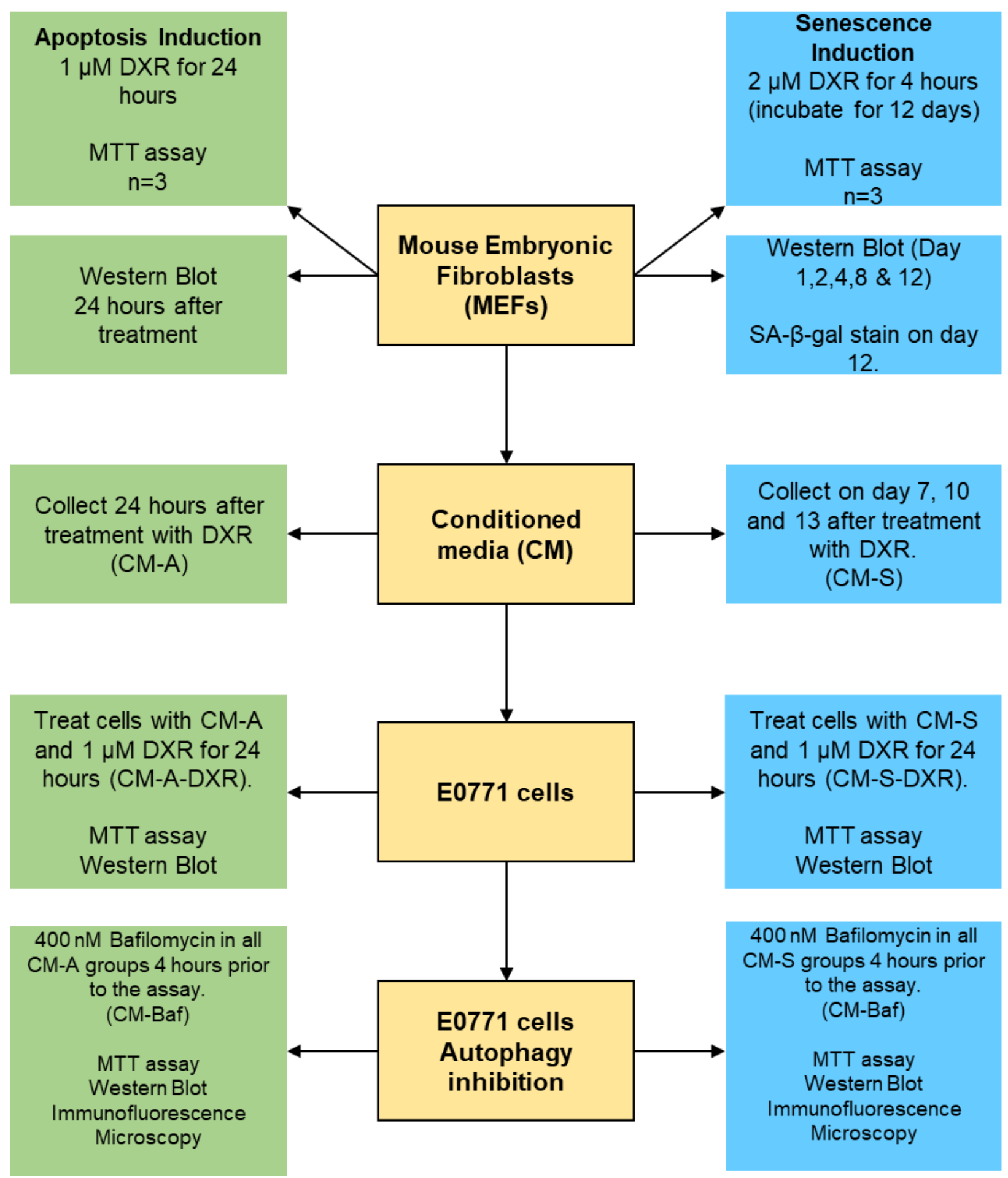


Figure 2.1: Study design. Conditioned media for treatment groups was generated by treating MEFs with DXR. E0771 cells were treated with the conditioned media groups and cell viability and apoptotic and autophagic activity was assessed.

\subsection{Cell culture}

MEFs and mouse E0771 breast cancer cells were used to perform experiments. The cells were cultured in Dulbecco's Modified Eagle's medium (DMEM), supplemented with 10\% Fetal Bovine Serum (FBS) and 1\% Penstrep (now referred to as growth medium). The cells were cultured in T75 flasks, respectively, and was maintained at $37^{\circ} \mathrm{C}$ in a $5 \% \mathrm{CO}_{2}$ incubator and were routinely sub-cultured at $70-80 \%$ confluency. Both cell lines were allowed to grow in the T75 flasks until they reached $80 \%$ confluency and were then split into the appropriate plates with new growth medium for various experiments. Subculturing of cells was achieved by removing the growth medium, followed by incubation with $4 \mathrm{ml} 0.25 \%$ Trypsin at $37^{\circ} \mathrm{C}$ until the cells were detached completely from the flask.

\subsection{Experimental protocol for MEFs}

\subsubsection{Treatment preparations}

MEFs were grown in T75 flasks and was categorized into six groups (Table 2.1). The following treatment protocols were performed to generate the desired groups of conditioned media (control, apoptosis and senescence) which was used to treat E0771 cells (refer to 2.2.4).

Table 2.1: MEFs were categorized into three groups and were treated accordingly to generate conditioned media.

\begin{tabular}{|l|l|l|}
\hline Group number & Group name & Treatment \\
\hline Group 1 & Conditioned media control (CM-C) & None \\
\hline Group 2 & Conditioned media apoptosis (CM-A) & $1 \mu \mathrm{M}$ DXR (24 hours) \\
\hline Group 3 & Conditioned media senescence (CM-S) & $2 \mu$ DXR (4 hours) \\
\hline
\end{tabular}

Doxorubicin hydrochloride was obtained from Sigma. A stock solution was prepared by dissolving the powder in pure DMEM and stored at $-20^{\circ} \mathrm{C}$. The desired concentration of DXR was diluted into complete growth media before each experiment. 
For apoptosis induction, upon reaching 80\% confluency, MEFS were split and 100000 MEFs were seeded into 6-well plates $\left(10 \mathrm{~cm}^{2}\right)$ for protein extraction (see section 2.3) and $5000 \mathrm{MEFs}$ were seeded into 48-well plates for 24 hours to determine cell viability (see 2.2.3). The following day, growth medium was removed and $1 \mu \mathrm{M}$ of DXR was added and removed after 24 hours, followed by a protein harvest.

For senescence induction, upon reaching 80\% confluency, 75000 MEFs were seeded into 6well plates $\left(10 \mathrm{~cm}^{2}\right)$ supplemented with growth medium. The next day, $2 \mu \mathrm{M}$ of DXR was added and removed after 4 hours. Fresh growth media was added to the wells and cells remained in the incubator. Protein harvests were performed on days 1, 2, 4, 8 and 12 after the DXR treatment was removed. The SA- $\beta$-Gal stain (Sigma) was performed on day 12 (Appendix A).

\subsubsection{Determination of cell death}

\subsubsection{MTT Assay}

To determine the viability of MEFs after the treatment period, an MTT assay (Mosmann, 1983) was performed. Viable cells with an active metabolism have $\mathrm{NAD}(\mathrm{P}) \mathrm{H}$-dependent oxidoreductase enzymes which has the ability to convert MTT (yellow tetrazole) into purple Formazan crystals which accumulates as an insoluble precipitate inside cells. Formazan crystals must therefore be solubilized before measuring absorbance. MEFs were categorized into groups and seeded into 48-well plates at a seeding density of 7000 cells per well and was incubated in $200 \mu \mathrm{l}$ growth medium for 24 hours. The following day, growth medium was aspirated and different concentrations of DXR was administered into the wells and the cells were incubated in a $5 \% \mathrm{CO}_{2}$ incubator for 24 hours at $37^{\circ} \mathrm{C}$. After the treatment period was completed, $0.1 \mathrm{mg} / \mathrm{ml}$ MTT solution (made up in $0.1 \mathrm{M} \mathrm{PBS}$ ) was added into the wells and incubated for one hour in a $5 \% \mathrm{CO}_{2}$ incubator. Cell viability was then determined by solubilizing Formazan in an Isopropanol-0.1\% Triton-X solution. The absorbance was then measured at $595 \mathrm{~nm}$ in a plate reading spectrophotometer. The number of viable cells was expressed as a percentage (\%) of the total number of cells. 


\subsubsection{Conditioned media}

As mentioned previously, different groups of conditioned media were generated and was used to treat E0771 cells. For control conditioned media (CM-C), $30 \mathrm{ml}$ of growth medium in a T75 flask (no cells) was incubated in a $5 \% \mathrm{CO}_{2}$ incubator for 24 hours. After the treatment duration was completed, the media was collected into $50 \mathrm{ml}$ Falcon tubes, snap frozen in liquid nitrogen and stored at $-80^{\circ} \mathrm{C}$.

For senescence conditioned media (CM-S), 563000 MEFs were seeded into T75 flasks (x2) and were grown overnight in $10 \mathrm{ml}$ growth medium in a $5 \% \mathrm{CO}_{2}$ incubator. Thereafter, the growth medium was removed and MEFs were treated with $2 \mu \mathrm{M}$ of DXR for four hours in a $5 \% \mathrm{CO}_{2}$ incubator and $\mathrm{CM}$ was collected on day 7, 10 and 13. Media was collected from the T75 flasks and centrifuged at $5000 \mathrm{rpm}$ for 10 minutes at $4^{\circ} \mathrm{C}$. The media was carefully transferred to a new tube and filtered with a $0.2 \mu \mathrm{m}$ filter. The CM was then snap frozen and stored at $-80^{\circ} \mathrm{C}$ until use.

For apoptosis CM (CM-A), 563000 MEFs were seeded into T75 flasks (x3) and was grown overnight in $10 \mathrm{ml}$ growth medium in a $5 \% \mathrm{CO}_{2}$ incubator. Thereafter, growth medium was removed and MEFs were treated with $1 \mu \mathrm{M}$ of DXR for 24 hours in a $5 \% \mathrm{CO}_{2}$ incubator. After the treatment period, CM was collected from the T75 flasks and centrifuged at $5000 \mathrm{rpm}$ for 10 minutes at $4^{\circ} \mathrm{C}$. The media was carefully transferred to a new tube and filtered with a 0.2 $\mu \mathrm{m}$ filter. The $\mathrm{CM}$ was then snap frozen and stored at $-80^{\circ} \mathrm{C}$ until use.

\subsection{Protein extraction and quantification}

Following treatment protocols mentioned in 2.2.1, cells drained and placed on ice. The cell monolayers were then washed twice with $1 \mathrm{ml}$ of ice-cold PBS (Appendix B). Total cell protein was extracted by incubating the cells in $1 \mathrm{ml}$ of modified radio-immuno precipitation (RIPA) buffer (Appendix C) containing $1 \times$ complete $^{\mathrm{TM}}$ protease inhibitor cocktail (Roche), $1 \mathrm{mM} \mathrm{NaF}$, $1 \mathrm{mM} \mathrm{Na}_{3} \mathrm{VO}_{4}$ and $1 \mathrm{mM} \mathrm{PMSF}$. A cell scraper was used to detach cells from the surface of the wells and the cells were transferred to chilled Eppendorf tubes. The suspension from triplicate wells were pooled and sonicated for 10 seconds at 5 amplitudes. After the foam settled, the suspension was centrifuged and the supernatant was carefully collected and placed into a new pre-cooled Eppendorf tube and stored at $-80^{\circ} \mathrm{C}$. Protein content was 
quantified using the Bradford protein determination method before the preparation of cell lysates.

\subsubsection{Bradford Assay}

A Bradford assay was performed to determine the concentration of protein lysates (Bradford, 1976). A standard curve consisting of six incremental concentrations was prepared from a 2 $\mathrm{mg} / \mathrm{ml}$ bovine serum albumin (BSA) solution. Bradford samples were prepared with $5 \mu \mathrm{l}$ of the protein lysate. Absorbance was measured on a spectrophotometer set to $595 \mathrm{~nm}$. The absorbencies were plotted on a graph generated with the standard curve in Microsoft Excel. All samples were prepared in duplicate. For protein quantification, whole cell lysates were thawed and were centrifuged at $4^{\circ} \mathrm{C}$ and $8000 \mathrm{rpm}$ for 2 minutes. A $200 \mathrm{mg} / \mathrm{ml} \mathrm{BSA}$ working solution was prepared and Bradford standards were prepared in duplicate. A complete step by step protocol for Bradford assay is included in Appendix D.

\subsubsection{Sample preparation}

After protein quantification (determined by Bradford assay), aliquots containing $50 \mu \mathrm{g}$ of protein were prepared for each sample. Lysates were diluted in Laemmli sample buffer (150 $\mu \mathrm{l}$ mercaptoethanol and $850 \mu \mathrm{l}$ sample buffer) (Appendix E) and boiled for 5 minutes at $95^{\circ} \mathrm{C}$. The prepared samples were stored at $-80^{\circ} \mathrm{C}$ for future analysis by western blotting.

\subsubsection{SDS-Page and western blot analysis}

Prepared lysates were separated on $12 \%$ gels by sodium dodecyl sulphate polyacrylamide gel electrophoresis (SDS-PAGE) (TGX Stain-Free ${ }^{\mathrm{TM}}$ FastCast $^{\mathrm{TM}}$ Acrylamide kit, Biorad). Molecular weights of specific bands were determined by loading $4 \mu \mathrm{l}$ of a protein marker ladder into the first lane of each gel. Previously prepared samples were dry boiled for 5 minutes at 95을 $\mathrm{C}$ before $50 \mu \mathrm{g}$ of protein was loaded into each well. For the first 10 minutes, proteins were separated at $90 \mathrm{~V}$, followed by approximately 90 minutes at $110 \mathrm{~V}$ (Mini Protean System, BioRad, Hercules). After electrophoresis, separated proteins were transferred (Trans-blot Turbo $^{\text {TM }}$ RTA Transfer Kit, PVDF Biorad) onto PVDF membranes and blocked in $5 \%$ fat free milk in TBS-Tween (TBS-T) for one hour at room temperature with gentle agitation to avoid non-specific binding before overnight incubation at $4^{\circ} \mathrm{C}$ with specific primary antibodies (Table 
2. 2) (diluted in TBS-T). The following day, membranes were washed in TBS-T three times for 5 minutes. After the washing step, membranes were incubated in anti-rabbit horseradish peroxidase-conjugated secondary antibody for 1 hour at room temperature on a roller. After secondary antibody incubation, membranes were washed 3 times for 5 minutes in TBS-T. Antibodies were detected using the ECL (Biorad) detection kit. Exposed bands were quantified using Image Lab software ${ }^{\mathrm{TM}}$ (Biorad). Exposed bands were quantified relative to the total protein present in each lane. A stain free total protein analysis was used. The technology uses a trihalo compound that is directly incorporated into the gel. After UV exposure, the compound modifies tryptophan residues in proteins which cause them to fluoresce. This fluorescent signal was then detected by a CCD camera, after the gel was transferred to a PVDF membrane. A complete step by step protocol for SDS-PAGE and western blot analysis is included in Appendix F.

Table 2.2: Antibodies used in western blot analysis for MEFs.

\begin{tabular}{|l|l|l|}
\hline Antibody & Size & Concentration \\
\hline Apoptosis & & \\
\hline Cleaved caspase 3 & $17 \mathrm{kDa}$ & $1: 1000$ \\
\hline Cleaved caspase 8 & $57 \mathrm{kDa}$ & $1: 1000$ \\
\hline Cleaved caspase 9 & $35-37 \mathrm{kDa}$ & $1: 500$ \\
\hline PARP & $89 \mathrm{kDa}$ & $1: 1000$ \\
\hline Senescence & & \\
\hline MCM2 & $102 \mathrm{kDa}$ & $1: 10000$ \\
\hline p53 & $53 \mathrm{kDa}$ & $1: 500$ \\
\hline p21 & $21 \mathrm{kDa}$ & $1: 500$ \\
\hline p16 & $16 \mathrm{kDa}$ & $1: 1000$ \\
\hline RB & $106 \mathrm{kDa}$ & $1: 1000$ \\
\hline
\end{tabular}

\subsection{Experimental protocol for E0771 cells}

\subsubsection{Treatment preparations}

E0771 cells were grown in T75 flasks until 80\% confluency and were split into 6-well plates for the appropriate experiments. Control, apoptosis and senescence conditioned media (CM) 
generated from MEFs (section 2.2.4) were used to treat E0771 cells, alongside with DXR. E0771 cells were categorized into 6 groups (Table 2.3). 470000 cells (per well) were seeded into 6-well plates and 7000 cells (per well) were seeded into 48-well plates (for cell viability) and incubated in growth medium for 24 hours in a $5 \% \mathrm{CO}_{2}$ incubator. The following day, growth media was aspirated and cells were treated with the appropriate generated CM for 24 hours in a $5 \% \mathrm{CO}_{2}$ incubator. Afterwards, cell viability (section 2.2.3.1) and protein extraction (section 2.3) were performed as mentioned above. Western blot analysis (section 2.3.3) was used to determine the expression of apoptotic and senescence markers in the E0771 cells (see table 2.2).

Table 2.3: E0771 cells were categorized into 6 groups and treated with conditioned media generated from MEFs.

\begin{tabular}{|l|l|l|l|}
\hline $\begin{array}{l}\text { Group } \\
\text { number }\end{array}$ & Group name & Treatment & Duration \\
\hline Group 1 & $\begin{array}{l}\text { Conditioned media Control } \\
(\text { CM-C) }\end{array}$ & None & 24 hours \\
\hline Group 2 & $\begin{array}{l}\text { Conditioned media Control \& } \\
\text { DXR (CM-C-DXR) }\end{array}$ & $1 \mu \mathrm{M}$ DXR & 24 hours \\
\hline Group 3 & $\begin{array}{l}\text { Conditioned media apoptosis } \\
\text { (CM-A) }\end{array}$ & None & 24 hours \\
\hline Group 4 & $\begin{array}{l}\text { Conditioned media apoptosis } \\
\text { \& DXR (CM-A-DXR) }\end{array}$ & $1 \mu \mathrm{M}$ DXR \\
\hline Group 5 & $\begin{array}{l}\text { Conditioned media } \\
\text { senescence (CM-S) }\end{array}$ & None & 24 hours \\
\hline Group 6 & $\begin{array}{l}\text { Conditioned media } \\
\text { senescence\& DXR (CM-S- } \\
\text { DXR) }\end{array}$ & $1 \mu \mathrm{M}$ DXR & 24 hours \\
\hline
\end{tabular}

\subsubsection{Determination of autophagic induction}

Autophagy was determined by analysing the expression of LC3 and p62. E0771 cells were grown in T75 flasks and were categorized into different treatment groups. After reaching confluency, 470000 cells per well were seeded into 6-well plates for protein extraction (see section 2.3) and 7000 cells were seeded into 48-well plates to determine the effects of autophagy on cell viability (see section 2.2.3.1). Bafilomycin (400 nM), an autophagy inhibitor, was added 4 hours prior to the above-mentioned experiments to measure autophagic flux. Bafilomycin prevents maturation of autophagic vacuoles by inhibiting fusion between autophagosomes and lysosomes through the inhibition of vacuolar $\mathrm{H}^{+} \mathrm{ATPase}$. 
Table 2.4: E0771 cells were categorized into 12 groups and treated with conditioned media generated from MEFs as well as Bafilomycin to determine autophagic flux.

\begin{tabular}{|c|c|c|c|}
\hline $\begin{array}{l}\text { Group } \\
\text { number }\end{array}$ & Group name & Treatment & Duration \\
\hline Group 1 & Conditioned media Control (CM-C) & None & 24 hours \\
\hline Group 2 & $\begin{array}{l}\text { Conditioned media Control \& Baf } \\
\text { (CM-C-Baf) }\end{array}$ & Baf $(400 \mathrm{nM})$ & $\begin{array}{l}24 \text { hours } \\
\text { Baf: } 4 \text { hours prior to } \\
\text { end of treatment }\end{array}$ \\
\hline Group 3 & $\begin{array}{l}\text { Conditioned media Control \& DXR } \\
\text { (CM-C-DXR) }\end{array}$ & $1 \mu \mathrm{M} D \mathrm{DXR}$ & 24 hours \\
\hline Group 4 & $\begin{array}{l}\text { Conditioned media Control \& DXR \& } \\
\text { Baf (CM-C-DXR-Baf) }\end{array}$ & $\begin{array}{l}1 \mu \mathrm{M} \mathrm{DXR} \\
400 \mathrm{nM} \mathrm{Baf}\end{array}$ & $\begin{array}{l}\text { DXR: } 24 \text { hours } \\
\text { Baf: } 4 \text { hours prior to } \\
\text { end of treatment }\end{array}$ \\
\hline Group 5 & $\begin{array}{l}\text { Conditioned media apoptosis (CM- } \\
\text { A) }\end{array}$ & None & 24 hours \\
\hline Group 6 & $\begin{array}{l}\text { Conditioned media apoptosis \& Baf } \\
\text { (CM-A-Baf) }\end{array}$ & Baf $(400 \mathrm{nM})$ & $\begin{array}{l}24 \text { hours } \\
\text { Baf: } 4 \text { hours prior to } \\
\text { end of treatment }\end{array}$ \\
\hline Group 7 & $\begin{array}{l}\text { Conditioned media apoptosis \& } \\
\text { DXR (CM-A-DXR) }\end{array}$ & $1 \mu \mathrm{M}$ DXR & 24 hours \\
\hline Group 8 & $\begin{array}{l}\text { Conditioned media apoptosis \& } \\
\text { DXR \& Baf (CM-A-DXR-Baf) }\end{array}$ & $\begin{array}{l}1 \mu \mathrm{M} \text { DXR } \\
400 \mathrm{nM} \text { Baf }\end{array}$ & $\begin{array}{l}\text { DXR: } 24 \text { hours } \\
\text { Baf: } 4 \text { hours prior to } \\
\text { end of treatment }\end{array}$ \\
\hline Group 9 & $\begin{array}{l}\text { Conditioned media senescence } \\
\text { (CM-S) }\end{array}$ & None & 24 hours \\
\hline Group 10 & $\begin{array}{l}\text { Conditioned media senescence \& } \\
\text { Baf (CM-S-Baf) }\end{array}$ & Baf $(400 \mathrm{nM})$ & $\begin{array}{l}24 \text { hours } \\
\text { Baf: } 4 \text { hours prior to } \\
\text { end of treatment }\end{array}$ \\
\hline Group 11 & $\begin{array}{l}\text { Conditioned media senescence \& } \\
\text { DXR (CM-S-DXR) }\end{array}$ & $1 \mu \mathrm{M}$ DXR & 24 hours \\
\hline Group 12 & $\begin{array}{l}\text { Conditioned media senescence \& } \\
\text { DXR \& Baf (CM-S-DXR-Baf) }\end{array}$ & $\begin{array}{l}1 \mu \mathrm{M} \text { DXR } \\
400 \mathrm{nM} \text { Baf }\end{array}$ & $\begin{array}{l}\text { DXR: } 24 \text { hours } \\
\text { Baf: } 4 \text { hours prior to } \\
\text { end of treatment }\end{array}$ \\
\hline
\end{tabular}




\subsubsection{Western blot analysis for autophagy and proliferation}

The protocol for the western blot analysis in this section is described in section 2.3. A seeding density of 470000 cells per well in 6-well plates was used for this experiment. After the cells were seeded into the appropriate plates, they were allowed to grow for 24 hours in a $5 \% \mathrm{CO}_{2}$ incubator. Afterwards, the cells were treated as indicated in Table 2.4. For the determination of autophagy, membranes were incubated overnight at $4^{\circ} \mathrm{C}$ with specific primary antibodies (diluted in TBS-T) (Table 2.5).

Table 2.5: Antibodies for autophagy and proliferation used in western blot analysis for E0771.

\begin{tabular}{|l|l|l|}
\hline Antibody & Size & Concentration \\
\hline Autophagy & & \\
\hline LC3 II & $17 \mathrm{kDa}$ & $1: 1000$ \\
\hline p62 & $62 \mathrm{kDa}$ & $1: 1000$ \\
\hline Proliferation & & \\
\hline Phosphorylated Akt & $60 \mathrm{kDa}$ & $1: 1000$ \\
\hline Total Akt & $60 \mathrm{kDa}$ & \\
\hline Phosphorylated ERK1/2 & $44 / 42 \mathrm{kDa}$ & $1: 2000$ \\
\hline Total ERK $1 / 2$ & $44 / 42 \mathrm{kDa}$ & $1: 1000$ \\
\hline
\end{tabular}

\subsubsection{LC3 and p62 Immunocytochemistry}

After reaching $80 \%$ confluency, 70000 cells were seeded onto glass coverslips in 6 -well plates and was treated with conditioned media (control, apoptosis and senescence) for 24 hours and Bafilomycin for 24 hours as previously described. The media was aspirated from the wells and placed onto microscope slides, followed by a PBS wash step ( 3 times). The cells were then fixed with $4 \%$ Formaldehyde for 10 minutes, followed by another fixation period with $0.1 \%$ Triton-X for 10 minutes. This was followed by another PBS wash step (3 times). Afterwards, the cells were blocked in $1 \%$ BSA for 60 minutes and was then incubated in primary antibody overnight at $4{ }^{\circ} \mathrm{C}$. After primary incubation, the cells were washed with PBS ( 3 times) and incubated in secondary antibody (Donkey anti-Rabbit IgG $(\mathrm{H}+\mathrm{L})$ Secondary Antibody Alexa Fluor 568 [ThermoFisher Scientific, Cat A10042]) prepared in PBS for 60 minutes, protected from light. Afterwards, nuclei were counterstained with $10 \mathrm{mg} / \mathrm{ml}$ Hoechst (1:200) prepared in PBS for 10 minutes. Followed by another wash step with PBS, the slides were mounted onto microscope slides. Once the mounting media was dry (approximately 30-60 minutes) the 
slides were stored at $-20^{\circ} \mathrm{C}$, protected from light, until it was imaged. A complete step by step protocol for Immunocytochemistry is included in Appendix G.

\subsubsection{Quantification of LC3 and p62 puncta}

Images were acquired, as z-stacks (average step-width of $0.8 \mu \mathrm{m}$ ), with the Olympus ${ }^{\circledR}$ Cell^R system on an Olympus $\AA^{\circledR}$ IX81 inverted fluorescence microscope (Olympus $\AA$, GMBH Japan) using the $60 X$ oil immersion objective and the $360 \mathrm{~nm}$ and $492 \mathrm{~nm}$ excitation filters, using a Xenon Arc lamp as excitation source for Hoechst and FITC, respectively A complete step by step protocol for image processing is included in Appendix $\mathrm{H}$.

Prior to quantification, Image z-stacks were pre-processed as follows: Z-stacks were 3D deconvoluted with Huygens Professional software (Version 18.10, Scientific Volume Imaging, http://svi.nl), using the CMLE algorithm, with a signal to noise ratio (SNR) of 20 and a maximum of 40 iterations. 2D maximum intensity projections were produced from deconvoluted z-stacks and adaptive Otsu thresholding was applied to each image with a threshold smoothing scale of 0.5 and a threshold correction factor of 2.0 (CellProfiler 3.1.5) ${ }^{1}$. LC3 and p62 positive structures were then quantified through particle counts using Fiji (Image J) software ${ }^{2}$. To enhance accuracy of particle counts in each region of interest (ROI), outlines were drawn on the original, non-binarized fluorescence images and superimposed over the thresholded binary images.

\subsection{Statistical Analysis}

All controls were normalized using Image Lab software ${ }^{\mathrm{TM}}$ and all values were expressed as a percentage of the untreated control. Results are presented as mean \pm Standard Error of the Mean (SEM). Different groups were compared by using one-way analysis of variance (ANOVA), followed by a Bonferroni post-hoc test. Graphpad Prism version 5.01 (Graphpad Software, Inc, CA, USA) was used to perform statistical analyses. A value of $p<0.05$ is considered to be statistically significant. 


\section{Chapter 3: Results}

\subsection{Introduction}

The first objective of this study was to identify a concentration of DXR that will induce senescence and apoptosis, respectively, in MEFs. A variety of methods were used including: MTT assays (cell viability), SA-B-gal assays and western blots. Secondly, the paracrine of effects of fibroblasts were assessed by treating E0771 breast cancer cells with conditioned media which was assessed by MTT assays and western blots. The final objective of this study was to determine the effects of autophagy inhibition on cancer cell death. These independent experiments were carried out to enhance the accuracy and repeatability of the acquired results.

\subsubsection{Dose response changes in cell viability following DXR treatment}

To determine suitable conditions for apoptosis induction in MEFs, an MTT assay was used to indirectly measure cell viability. MEFs were treated with $0.1 \mu \mathrm{M}, 0.5 \mu \mathrm{M}, 1 \mu \mathrm{M}, 2 \mu \mathrm{M}, 4 \mu \mathrm{M}, 8$ $\mu \mathrm{M}$ and $10 \mu \mathrm{M} \mathrm{DXR}$ for 24 hours. Based on the dose response experiment, $1 \mu \mathrm{M}$ to $10 \mu \mathrm{M}$ DXR treatment showed a significant $(p<0.001)$ decrease in cell viability compared to the control (Figure 3.1).

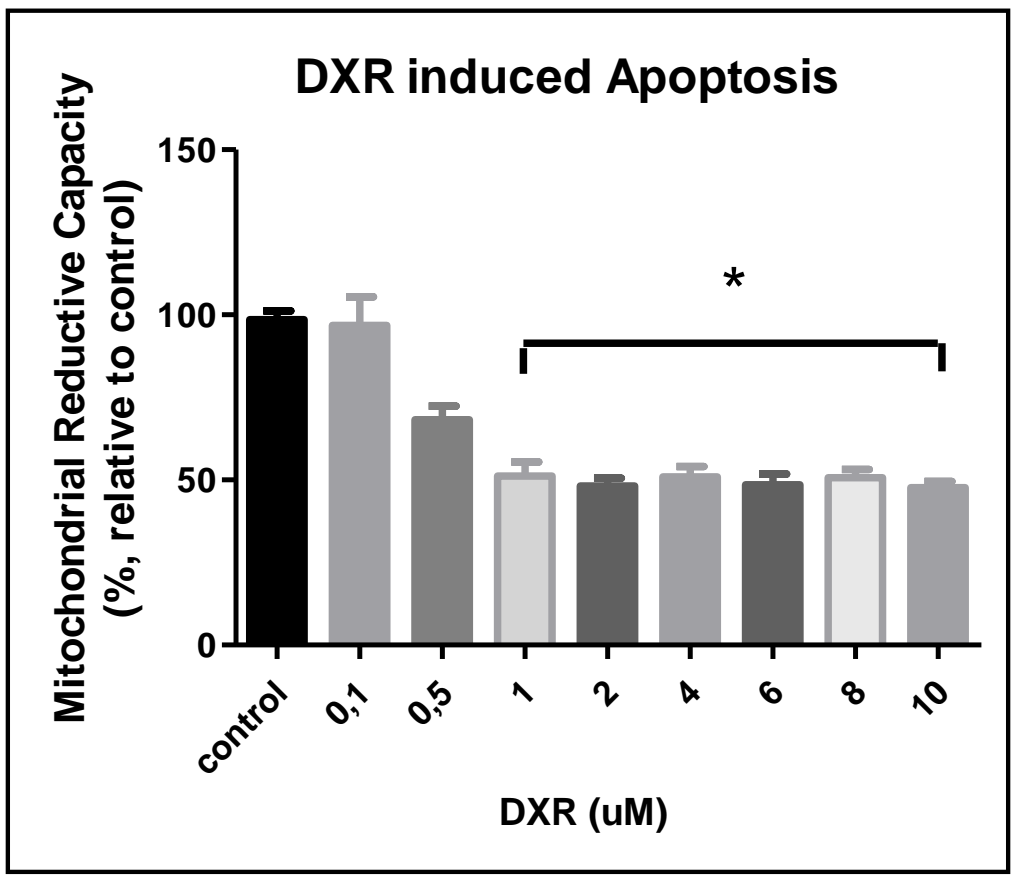


Figure 3.1: Percentage viability of MEFs after a DXR treatment period determined by an MTT assay. MEFs were seeded and treated with different DXR concentrations $(0.1 \mu \mathrm{M}, 0.5 \mu \mathrm{M}, 1 \mu \mathrm{M}, 2 \mu \mathrm{M}$, $4 \mu \mathrm{M}, 8 \mu \mathrm{M}$ and $10 \mu \mathrm{M})$ over a period of 24 hours. Results are presented as means $\pm \operatorname{SEM}(n=3)$. The asterisk $\left(^{*}\right)$ denotes a significant decrease in cell viability when compared to the control $(p<0.001)$. The percentage viable cells after the treatment period were compared with the percentage untreated viable cells.

\subsubsection{Apoptosis induction in MEFs with DXR}

\subsubsection{Caspase 8 cleavage to determine apoptosis activation}

Caspase 8 is situated in the cytosol, in its inactive form ( $55 \mathrm{kDa})$ and becomes activated during the extrinsic apoptotic pathway (Kominami et al., 2012). Once activated, procaspase 8 is split into large $(20 \mathrm{kDa})$ and small $(12 \mathrm{kDa})$ polypeptides (Kruidering \& Evan, 2000). Activated caspase 8 is then released into the cytosol to cleave downstream effector caspases like caspase 3 . After the 24 -hour treatment with DXR, cleaved caspase 8 expression significantly increased in the treatment group $(p<0.05)$ (Figure 3.2).

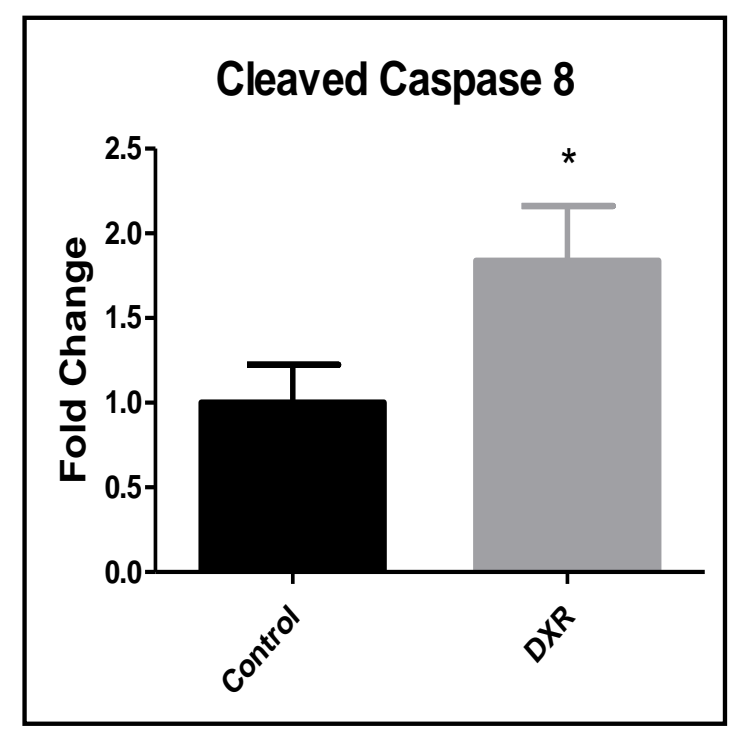




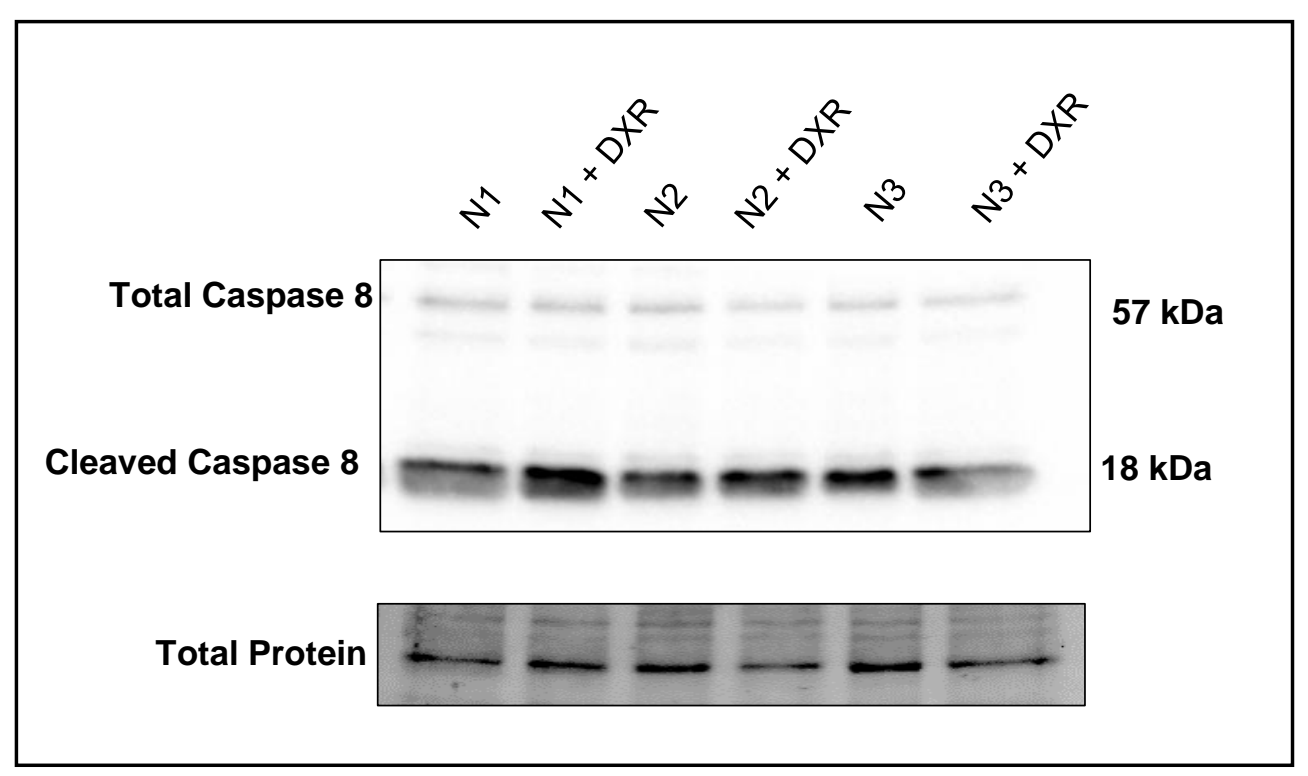

Figure 3.2: Western blot analysis to detect caspase 8 cleavage. MEFs were seeded and treated with DXR $(1 \mu \mathrm{M})$ for 24 hours. Proteins $(50 \mu \mathrm{g})$ from MEFs were separated by SDS-PAGE and transferred to a PVDF membrane for western blot analysis. Caspase 8 expression is shown for untreated control MEFs (lanes 1, 3 and 5) and MEFs treated with $1 \mu \mathrm{M}$ DXR (lanes 2, 4 and 6). Results are presented as means $\pm \operatorname{SEM}(n=3)$. The asterisk $\left(^{*}\right)$ denotes a significant increase in cleaved caspase 8 expression relative to the control $(p<0.05)$.

\subsubsection{Caspase 9 cleavage to determine apoptosis activation}

Caspase 9 is referred to as an initiator caspase, involved in the intrinsic (mitochondrial) apoptotic pathway (Sharifi et al., 2015). The apoptosome activates procaspase 9, which in turn stimulates the activation of caspase 3 or caspase 7 to induce apoptosis (Twiddy \& Cain, 2007). There were no significant changes in caspase 9 expression after DXR treatment $(p>0.05)$ and no cleavage was observed (Figure 3.2). 

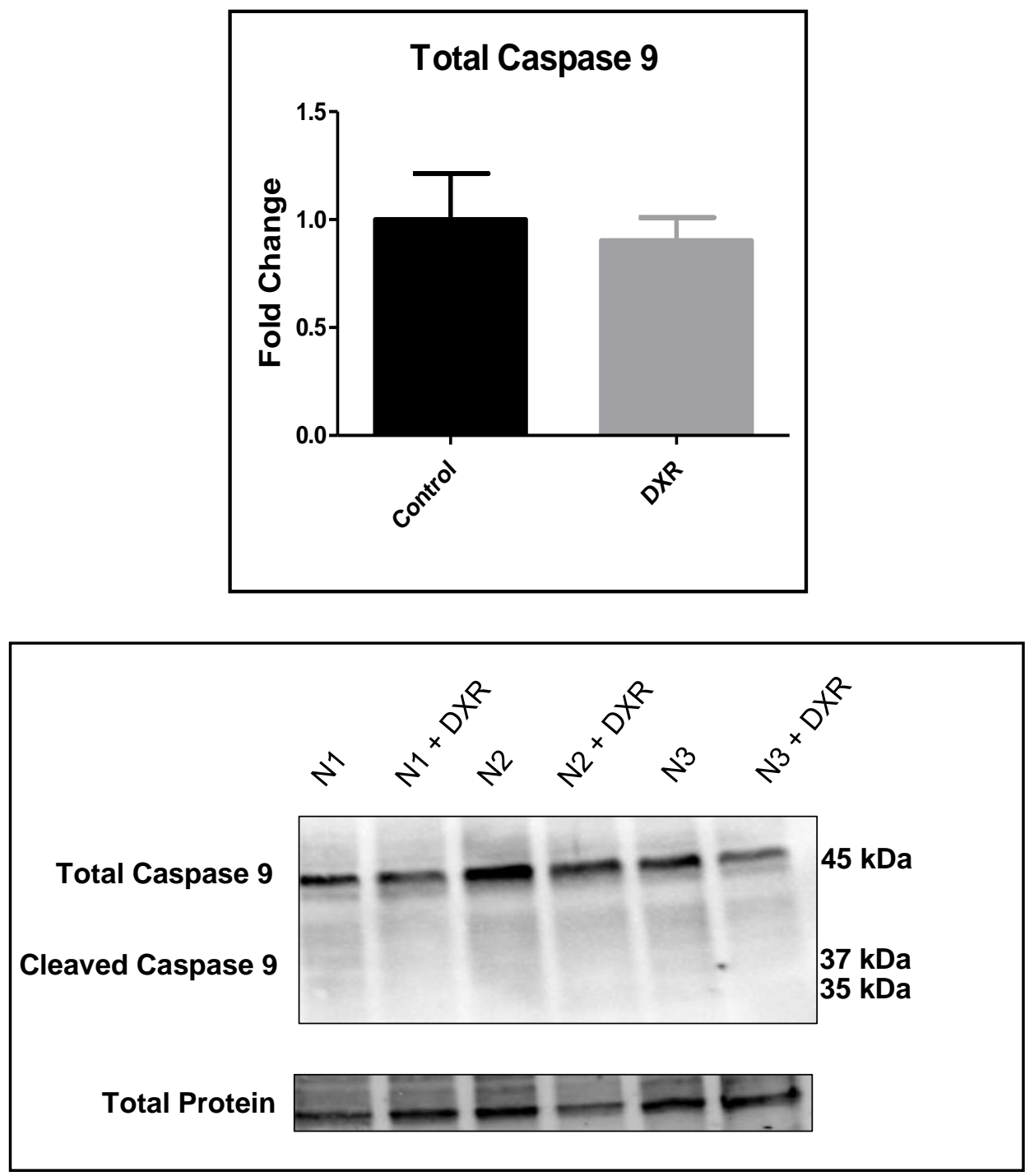

Figure 3.3: Western blot analysis to detect caspase 9 cleavage. MEFs were seeded and treated with DXR $(1 \mu \mathrm{M})$ for 24 hours. Proteins $(50 \mu \mathrm{g})$ from MEFs were separated by SDS-PAGE and transferred to a PVDF membrane for western blot analysis. Caspase 9 expression is shown for untreated control MEFs (lanes 1, 3 and 5) and MEFs treated with $1 \mu \mathrm{M}$ DXR (lanes 2, 4 and 6). Results are presented as means \pm SEM $(n=3)$. No significant changes were observed in caspase 9 expression $(p>0.05)$. 


\subsubsection{Caspase 3 cleavage to confirm apoptosis activation}

Caspase 3 is activated by both apoptotic pathways (the mitochondrial intrinsic pathway and extrinsic death receptor pathway). Caspase 3 is an executioner caspase and only becomes active once it is cleaved and activated by initiator caspases after apoptotic signalling. Membranes were probed with caspase 3 to determine the ability of DXR to induce apoptosis in MEFs. Cleaved caspase 3 significantly increased after the treatment period $(p<0.05)$ (Figure 3.4).
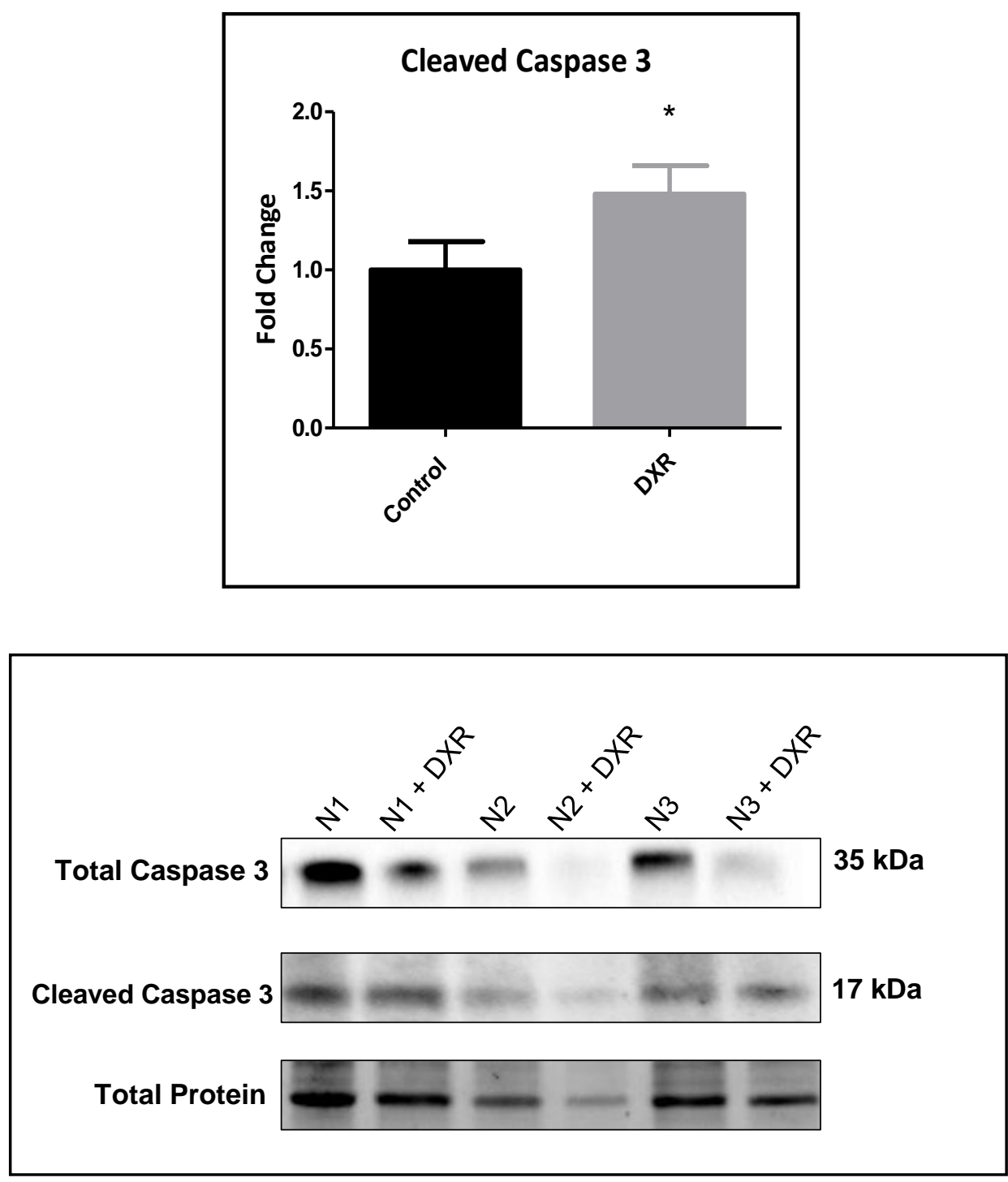

Figure 3.4: Western blot analysis to detect caspase 3 cleavage. MEFs were seeded and treated with DXR $(1 \mu \mathrm{M})$ for 24 hours. Proteins $(50 \mu \mathrm{g})$ from MEFs were separated by SDS-PAGE and transferred to a PVDF membrane for western blot analysis. Caspase 3 expression is shown for untreated control MEFs (lanes 1, 3 and 5) and MEFs treated with $1 \mu \mathrm{M} \mathrm{DXR}$ (lanes 2, 4 and 6). Results are presented as means $\pm \operatorname{SEM}(n=3)$. The asterisk $\left(^{*}\right)$ denotes a significant increase in cleaved caspase 3 expression relative to the control $(p<0.05)$. 


\subsubsection{Poly (ADP-ribose) polymerase cleavage to confirm apoptosis activation}

PARP belongs to a family of proteins that is involved in various cellular processes like programmed cell death and DNA repair (Herceg and Wang, 2001). To determine the ability of DXR to induce apoptosis in MEFs and to confirm the apoptotic results obtained in the caspase 3 western blots, the expression of PARP was investigated. After the 24-hour treatment period with DXR, PARP cleavage decreased significantly relative to the control $(p<0.05)$. Total PARP significantly decreased $(p<0.05)$ and the Cleaved/total PARP ratio significantly $(p<0.001)$ decreased relative to the control (Figure 3.5).
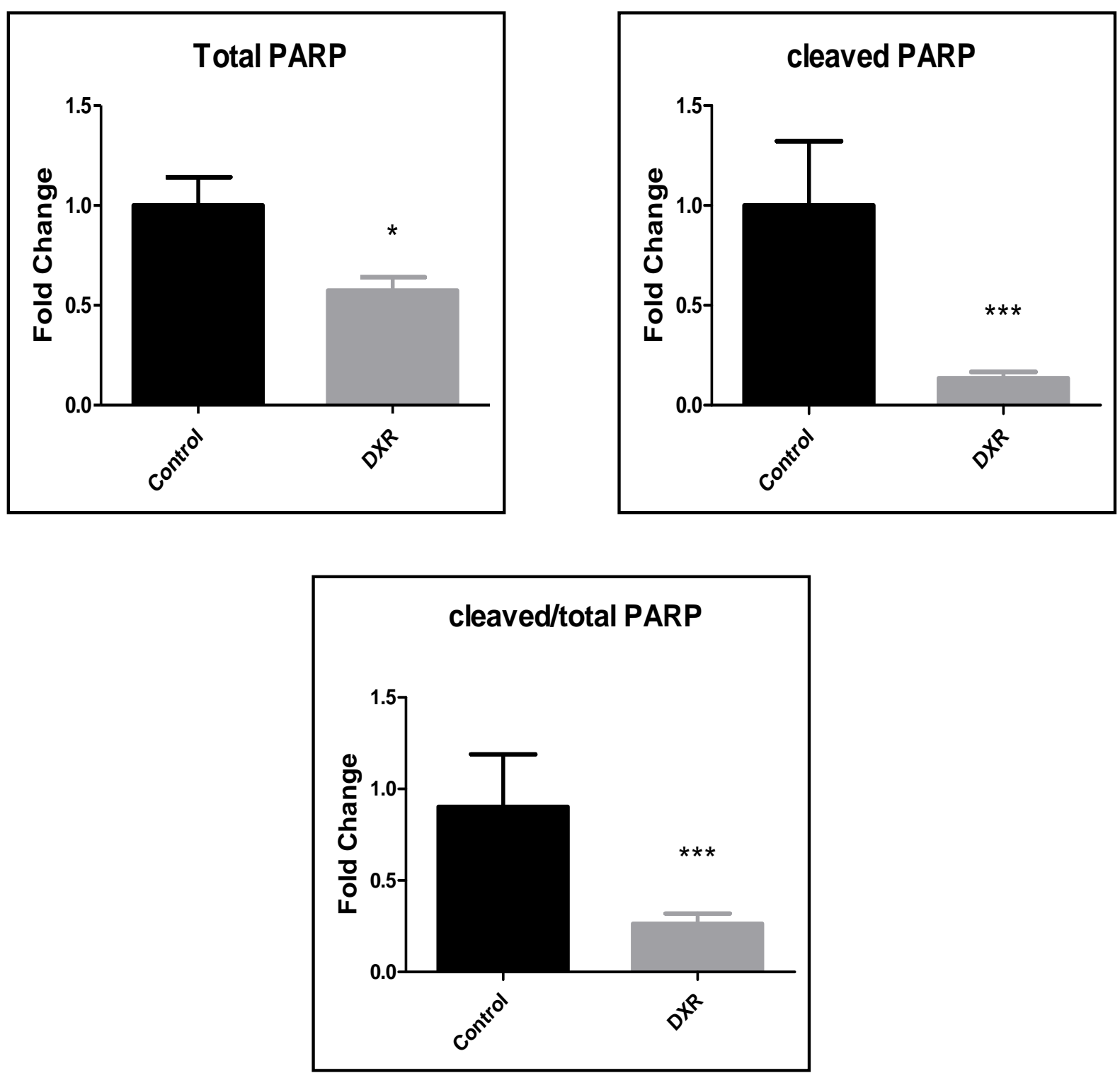


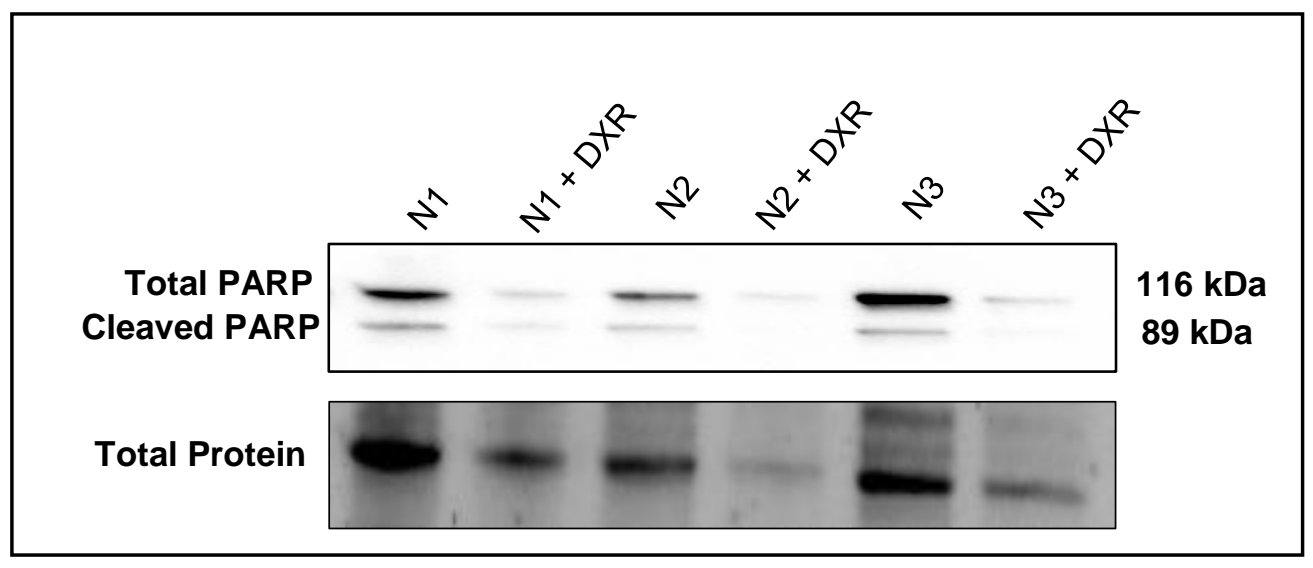

Figure 3.5: Western blot analysis to detect PARP cleavage. MEFs were seeded and treated with DXR $(1 \mu \mathrm{M})$ for 24 hours. Proteins $(50 \mu \mathrm{g})$ from MEFs were separated by SDS-PAGE and transferred to a PVDF membrane for western blot analysis. PARP expression is shown for untreated control MEFs (lanes 1, 3 and 5) and MEFs treated with $1 \mu \mathrm{M}$ DXR (lanes 2, 4 and 6). Results are presented as means \pm SEM $(n=3)$. The asterisk ( $\left.{ }^{*}\right)$ denotes a significant decrease in total PARP expression relative to the control $(p<0.05)$, a significant decrease in cleaved PARP $(p<0.001)$ and a significant decrease in the Cleaved/total PARP ratio $(p<0.001)$.

\subsection{Determination of senescence in MEFs after DXR treatment}

\subsubsection{Senescence associated B-galactosidase assay}

Senescence-associated beta-galactosidase is a hydrolase enzyme that catalyses the hydrolysis of $\beta$-galactosides into monosaccharides in senescent cells. In senescent cells, endogenous lysosomal beta-galactosidase is accumulated and overexpressed. When betagalactosidase assays are carried out at $\mathrm{pH} 6.0$, cells that are in senescence develop a bluecoloured stain that results from the cleavage of chromogenic substrate X-gal. MEFs were treated with DXR for 4 hours and incubated for 12 days. After the incubation period, the number of senescent cells in the fibroblast population was expressed as a percentage relative to the number of cells that were not stained. Table 3.1 indicates the average amount of senescent cells present in a cell population. 
Table 3.1: The average amount of senescent cells present in a randomly selected cell population (MEFs).

\begin{tabular}{|c|c|c|c|c|}
\hline \multicolumn{5}{|l|}{ N1 } \\
\hline Image & Untreated MEFs & $\begin{array}{l}\text { Total } \\
\text { cells }\end{array}$ & Senescent cells & $\%$ \\
\hline 1 & 53 & 23 & 14 & 60,87 \\
\hline 2 & 56 & 37 & 21 & 56,76 \\
\hline 3 & 50 & 33 & 17 & 51,52 \\
\hline 4 & 48 & 29 & 14 & 48,28 \\
\hline 5 & 60 & 22 & 11 & 50,00 \\
\hline 6 & 56 & 21 & 11 & 52,38 \\
\hline \multicolumn{5}{|l|}{ N2 } \\
\hline Image & Untreated MEFs & $\begin{array}{l}\text { Total } \\
\text { cells }\end{array}$ & Senescent cells & $\%$ \\
\hline 1 & 44 & 23 & 14 & 60,87 \\
\hline 2 & 56 & 22 & 11 & 50,00 \\
\hline 3 & 53 & 21 & 13 & 61,90 \\
\hline 4 & 58 & 21 & 11 & 52,38 \\
\hline 5 & 45 & 20 & 10 & 50,00 \\
\hline 6 & 52 & 16 & 10 & 62,50 \\
\hline \multicolumn{5}{|l|}{ N3 } \\
\hline Image & Untreated MEFs & $\begin{array}{l}\text { Total } \\
\text { cells }\end{array}$ & Senescent cells & $\%$ \\
\hline 1 & 51 & 29 & 13 & 44,83 \\
\hline 2 & 53 & 30 & 20 & 66,67 \\
\hline 3 & 45 & 32 & 25 & 78,13 \\
\hline 4 & 48 & 31 & 15 & 48,39 \\
\hline 5 & 51 & 26 & 16 & 61,54 \\
\hline \multirow[t]{2}{*}{6} & 52 & 28 & 16 & 57,14 \\
\hline & & \multicolumn{3}{|c|}{ Senescent population: $56,34 \%$} \\
\hline
\end{tabular}

Based on the experiment, the average senescent population can be referred to as nonsenescent (population < 80\%) (Figure 3.6). 

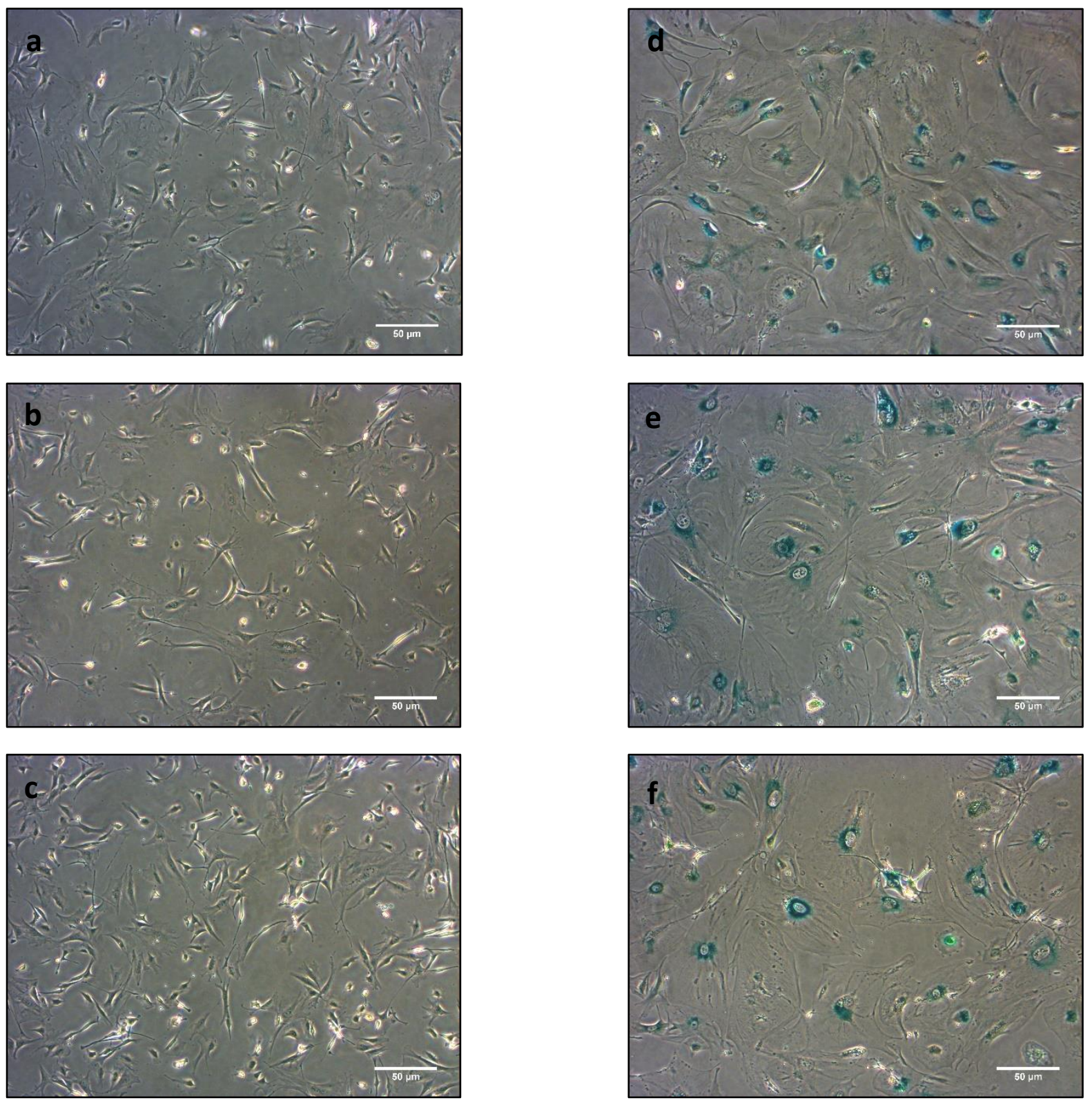

Figure 3.6: Phenotypical changes in MEFs following treatment with DXR for senescence induction. MEFs were seeded and treated with DXR $(2 \mu \mathrm{M})$ for 4 hours (a-c). Following the treatment period, MEFs were incubated for 12 days in a $5 \% \mathrm{CO}_{2}$ incubator at $37^{\circ} \mathrm{C}$. MEFs were stained on day 12 for 16 hours before acquiring the images with a bright field microscope (d-f). 


\subsubsection{Senescence induction in MEFs after DXR treatment}

\subsubsection{Mini chromosome Maintenance Complex Component 2 (MCM2) Expression in senescent MEFs}

MCM2 is a human nuclear protein that plays an important role in the onset of DNA replication and cell division during the cell cycle. Membranes were incubated in MCM2 overnight as an indication of the proliferative state of the cells, since senescent cells can no longer proliferate. There was a significant $(p<0.05)$ decrease in MCM2 expression on day 8 and 12 when compared to the control, as well as when compared to day $1(p<0.05)$ (Figure 3.7).
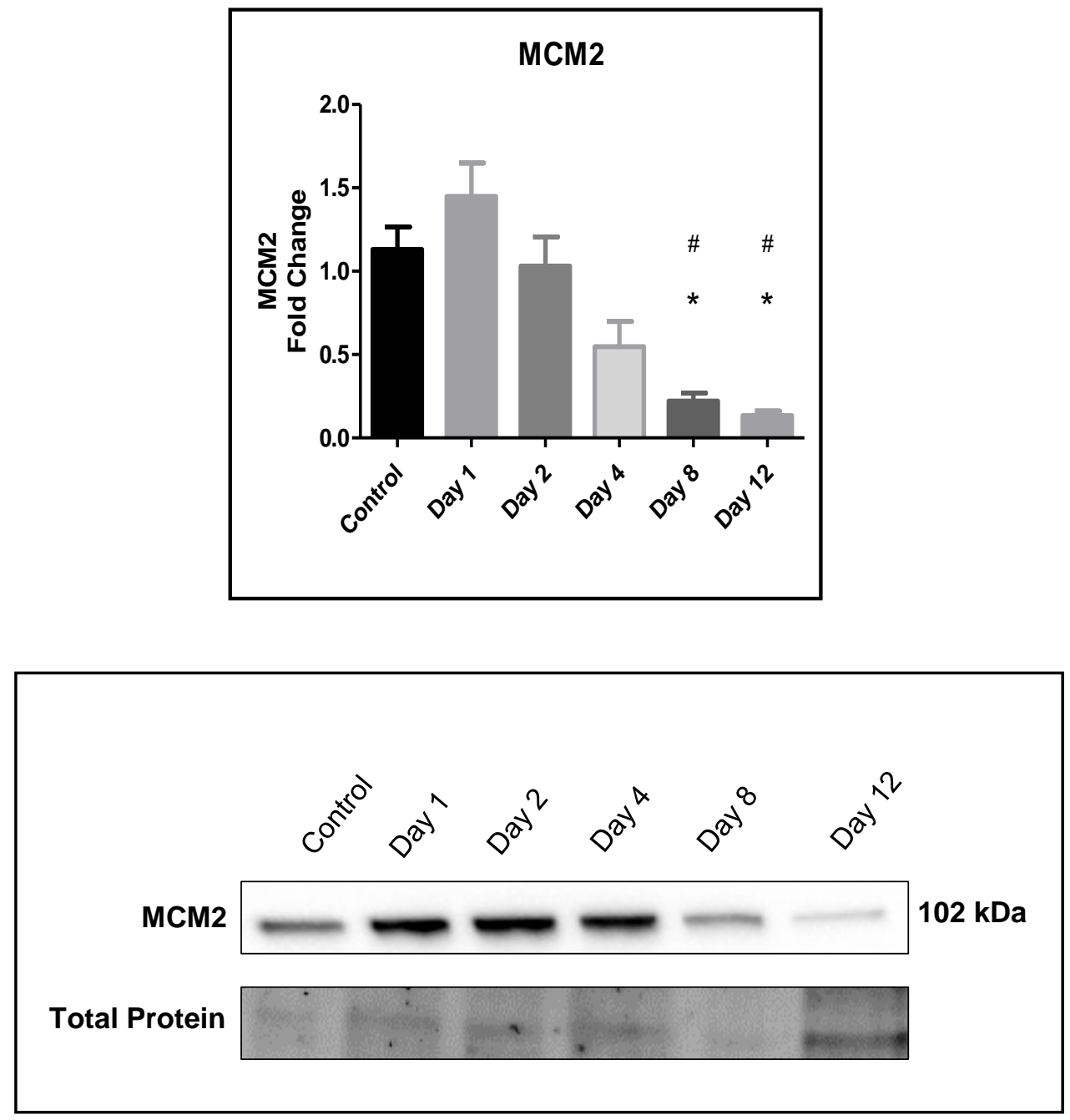

Figure 3.7: Western blot analysis to detect MCM2 expression. MEFs were seeded and treated with DXR $(2 \mu \mathrm{M})$ for 4 hours. After treatment, MEFs were incubated in a $5 \% \mathrm{CO}_{2}$ incubator at $37^{\circ} \mathrm{C}$ for 1,2 , 4,8 and 12 days respectively. Proteins $(50 \mu \mathrm{g})$ from MEFs were separated by SDS-PAGE and transferred to a PVDF membrane for western blot analysis. MCM2 expression is shown for untreated control MEFs (lane 1) and MEFs treated with $2 \mu \mathrm{M}$ DXR for 4 hours on Day 1 (lane 2), Day 2 (lane 3), Day 4 (lane 4), Day 8 (lane 5) and Day 12 (lane 6). Results are presented as means \pm SEM $(n=3)$. The 
asterisk $\left({ }^{*}\right)$ denotes a significant decrease in MCM2 expression relative to the control $(p<0.05)$ and $(\#)$ denotes a significant decrease when compared to Day $1(p<0.05)$.

\subsubsection{2 p53 Expression in senescent MEFs}

p53 is a tumour suppressor protein involved in both the apoptotic and cell growth arrest (senescence). The expression of p53 was analysed to determine whether DXR induced senescence in MEFs. During the treatment periods, ranging from days 1 to 12, p53 expression significantly $(p<0.05)$ decreased on day 8 and 12 when compared to day 1 . The expression of p53 was also significantly decreased $(p<0.05)$ when compared to both the control and day 2 (Figure 3.8).
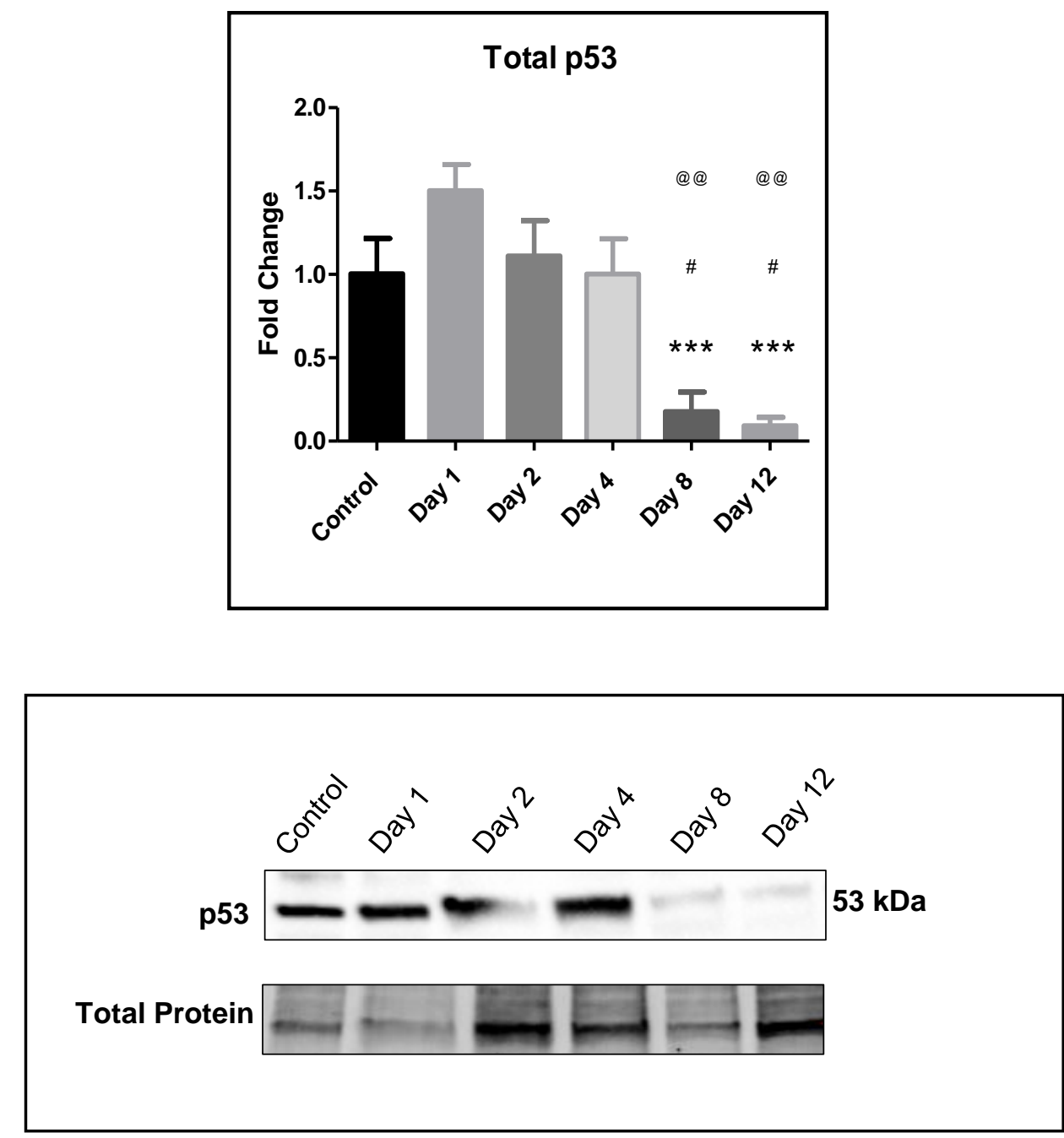

Figure 3.8: Western blot analysis to detect p53 expression. MEFs were seeded and treated with DXR $(2 \mu \mathrm{M})$ for 4 hours. After treatment, MEFs were incubated in a $5 \% \mathrm{CO}_{2}$ incubator at $37^{\circ} \mathrm{C}$ for 1,2 , 4,8 and 12 days respectively. Proteins $(50 \mu \mathrm{g})$ from MEFs were separated by SDS-PAGE and 
transferred to a PVDF membrane for western blot analysis. p53 expression is shown for untreated control MEFs (lane 1) and MEFs treated with $2 \mu \mathrm{M}$ DXR for 4 hours on day 1 (lane 2), day 2 (lane 3), day 4 (lane 4), day 8 (lane 5 ) and day 12 (lane 6 ). Results are presented as means \pm SEM $(n=3)$. The asterisk $\left(^{*}\right)$ denotes a significant decrease in p53 expression relative to the control $(p<0.001)$; (@) denotes a significant decrease relative to Day $1(p<0.01)$ and $(\#)$ denotes a significant decrease in $p 53$ expression when compared to Day $2(p<0.05)$.

\subsubsection{3 p21 Expression in senescent MEFs}

p21 is a cyclin-dependent kinase inhibitor that functions as a sensor and effector of many antiproliferative signals, thus promoting cell cycle arrest in response to many stimuli. The expression of p21 increased significantly $(p<0.001)$ on day 12 when compared to the control. p21 expression was also significantly higher on day 12 when compared to day 1, 2, 4 and 8 $(p<0.01)$.
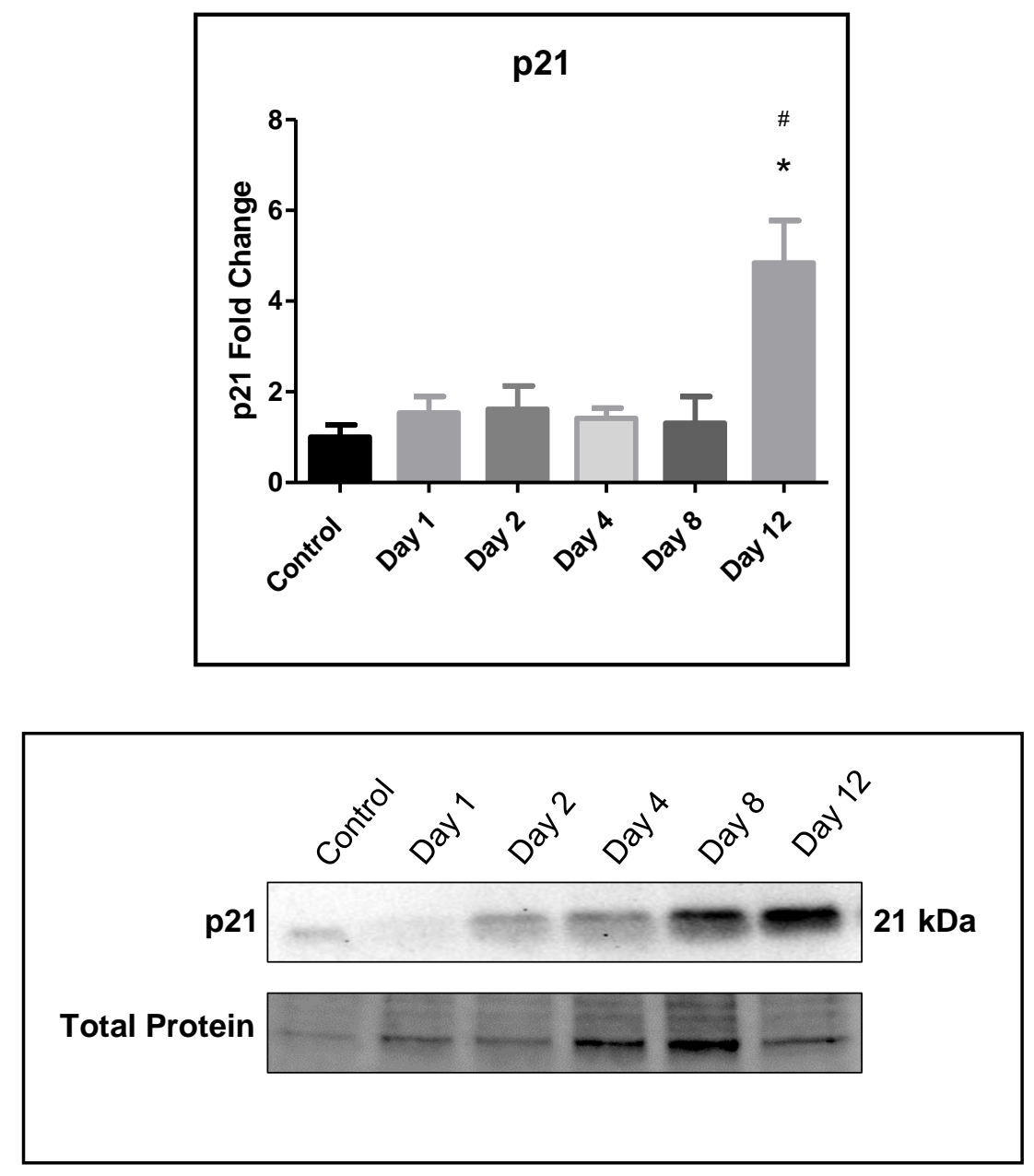

Figure 3.9: Western blot analysis to detect p21 expression. MEFs were seeded and treated with DXR $(2 \mu \mathrm{M})$ for 4 hours. After treatment, MEFs were incubated in a $5 \% \mathrm{CO}_{2}$ incubator at $37^{\circ} \mathrm{C}$ for 1,2 , 
4, 8 and 12 days respectively. Proteins $(50 \mu \mathrm{g})$ from MEFs were separated by SDS-PAGE and transferred to a PVDF membrane for western blot analysis. p21 expression is shown for untreated control MEFs (lane 1) and MEFs treated with $2 \mu \mathrm{M}$ DXR for 4 hours on day 1 (lane 2), day 2 (lane 3), day 4 (lane 4), day 8 (lane 5) and day 12 (lane 6 ). Results are presented as means \pm SEM $(n=3)$. The asterisk $\left(^{*}\right)$ denotes a significant increase in $\mathrm{p} 21$ expression relative to the control $(p<0.001)$; (\#) denotes a significant increase relative to day $1,2,4$ and $8(p<0.01)$.

\subsubsection{4 p16 Expression in senescent MEFs}

p16 contributes to cell cycle regulation by inhibiting the synthesis-phase (S-phase) during the cell cycle. p16 binds to CDK4/6 and inhibits CDK4/6-mediated phosphorylation of Retinoblastoma $(R B)$ family members. The expression of $p 16$ significantly $(p<0.01)$ increased on day 12 when compared to the control (Figure 3.10). Additionally, p16 expression significantly increased $(\mathrm{p}<0.05)$ on day 12 when compared to day 1 and day 2 . On day $8, p 16$ expression was also significantly increased $(p<0.05)$ when compared to the control.
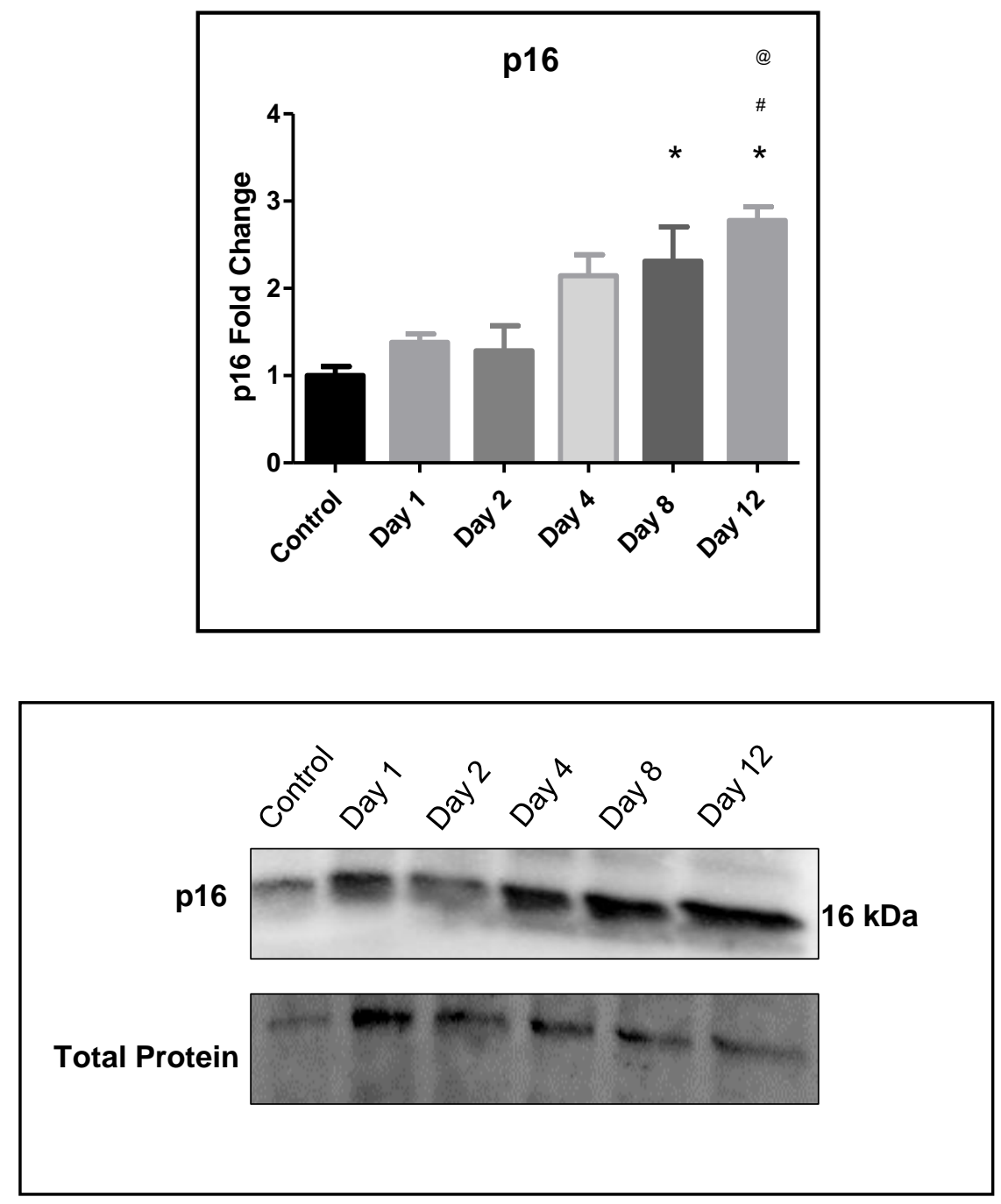
Figure 3.10: Western blot analysis to detect p16 expression. MEFs were seeded and treated with DXR $(2 \mu \mathrm{M})$ for 4 hours. After treatment, MEFs were incubated in a $5 \% \mathrm{CO}_{2}$ incubator at $37^{\circ} \mathrm{C}$ for 1,2 , 4,8 and 12 days respectively. Proteins $(50 \mu \mathrm{g})$ from MEFs were separated by SDS-PAGE and transferred to a PVDF membrane for western blot analysis. p16 expression is shown for untreated control MEFs (lane 1) and MEFs treated with $2 \mu \mathrm{M}$ DXR for 4 hours on day 1 (lane 2), day 2 (lane 3), day 4 (lane 4), day 8 (lane 5) and day 12 (lane 6 ). Results are presented as means \pm SEM $(n=3)$. The asterisk ( $\left.{ }^{*}\right)$ denotes a significant increase in $p 16$ expression relative to the control $(p<0.05)$; (\#) denotes a significant increase relative to Day $1(p<0.05)$ and $(@)$ denotes a significant increase relative to day $2(p<0.05)$.

\subsubsection{Total Retinoblastoma Expression in senescent MEFs}

The Retinoblastoma (RB) protein is known as a tumour suppressor protein that prevents excessive cell growth by inhibiting cell cycle progression until the cell is ready to divide. When the cell is able to divide, RB is phosphorylated and becomes inactive, allowing the cell cycle to continue. Membranes were probed with RB to confirm cell cycle inhibition. RB expression significantly increased $(p<0.001)$ when compared to the control as well as day $1,2,4$ and 8 (Figure 3.11).
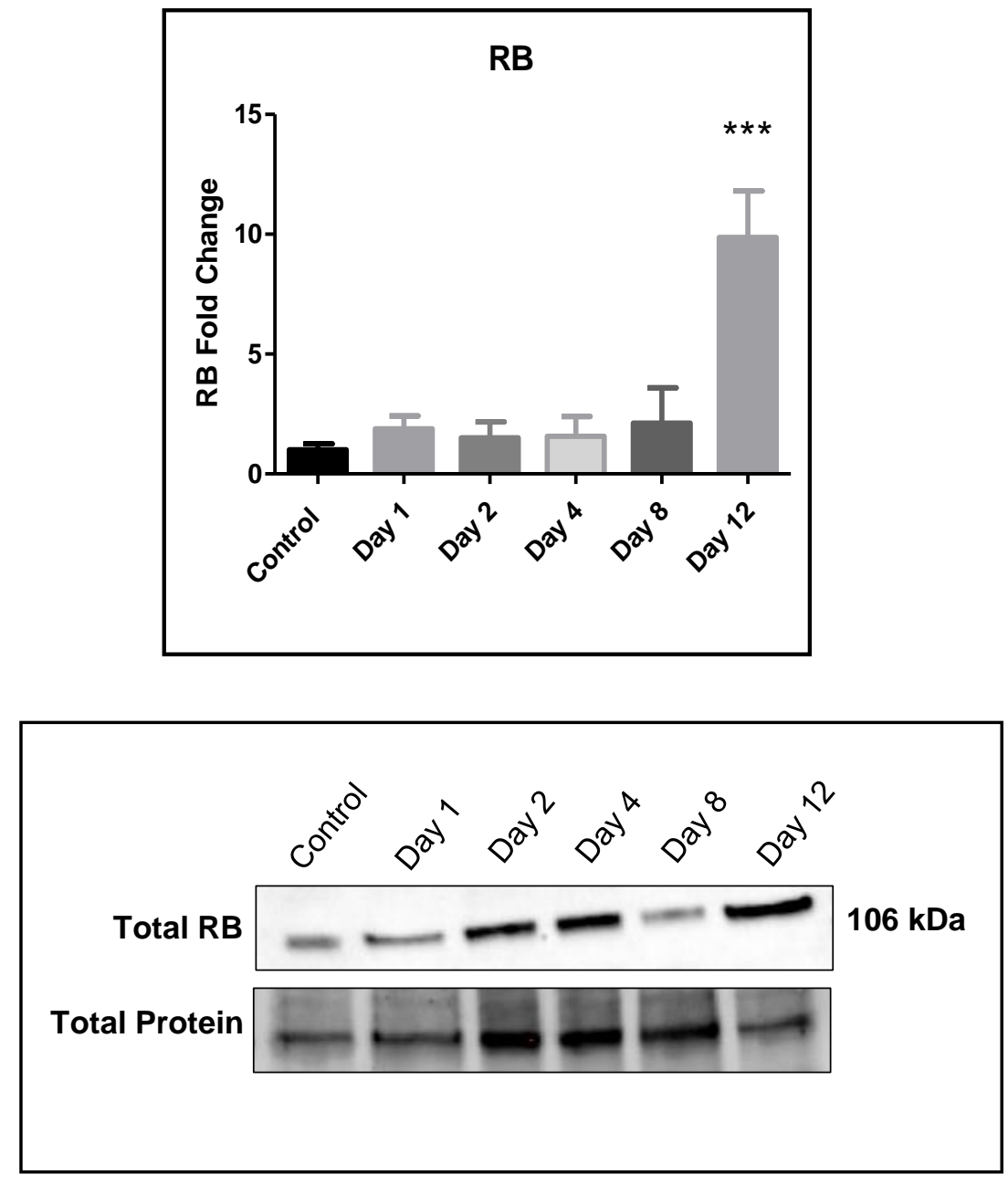
Figure 3.11: Western blot analysis to detect RB expression. MEFs were seeded and treated with DXR $(2 \mu \mathrm{M})$ for 4 hours. After treatment, MEFs were incubated in a $5 \% \mathrm{CO}_{2}$ incubator at $37^{\circ} \mathrm{C}$ for 1,2 , 4,8 and 12 days respectively. Proteins $(50 \mu \mathrm{g})$ from MEFs were separated by SDS-PAGE and transferred to a PVDF membrane for western blot analysis. RB expression is shown for untreated control MEFs (lane 1) and MEFs treated with $2 \mu \mathrm{M}$ DXR for 4 hours on day 1 (lane 2), day 2 (lane 3), day 4 (lane 4), day 8 (lane 5) and day 12 (lane 6 ). Results are presented as means $\pm \operatorname{SEM}(n=3)$. The asterisk $\left(^{*}\right)$ denotes a significant increase in RB expression relative to the control as well as day 1, 2, 4 and $8(p<0.001)$.

\subsection{Paracrine effects of senescent and apoptotic MEFs on E0771 cells}

E0771 cancer cells were exposed to CM from apoptotic and senescent MEFs to assess the effects of paracrine factors released from these cells on breast cancer cells, with or without the presence of DXR. Cell viability was indirectly confirmed with an MTT assay and western blots were used to determine apoptotic and senescent protein expression. Furthermore, autophagy was also inhibited in the E0771 cells with Bafilomycin to determine the effects on cell viability.

\subsubsection{Changes in cell viability following Conditioned media and DXR treatment}

An MTT assay was used to indirectly evaluate cell death when E0771 cells were incubated in CM obtained from MEFs. The control CM with added DXR (CM-C-DXR, $1 \mu \mathrm{M})$ significantly $(p<0.001)$ decreased E0771 cell viability when compared to the control CM (CM-C). CM induced apoptosis with DXR $(C M-A-D X R)$ decreased significantly $(p<0.005)$ from the conditioned media apoptosis group (CM-A). CM induced senescence with DXR (CM-S-DXR) had a significant increase $(p<0.05)$ when compared to CM-C-DXR and CM-A-DXR (Figure 3.12). 


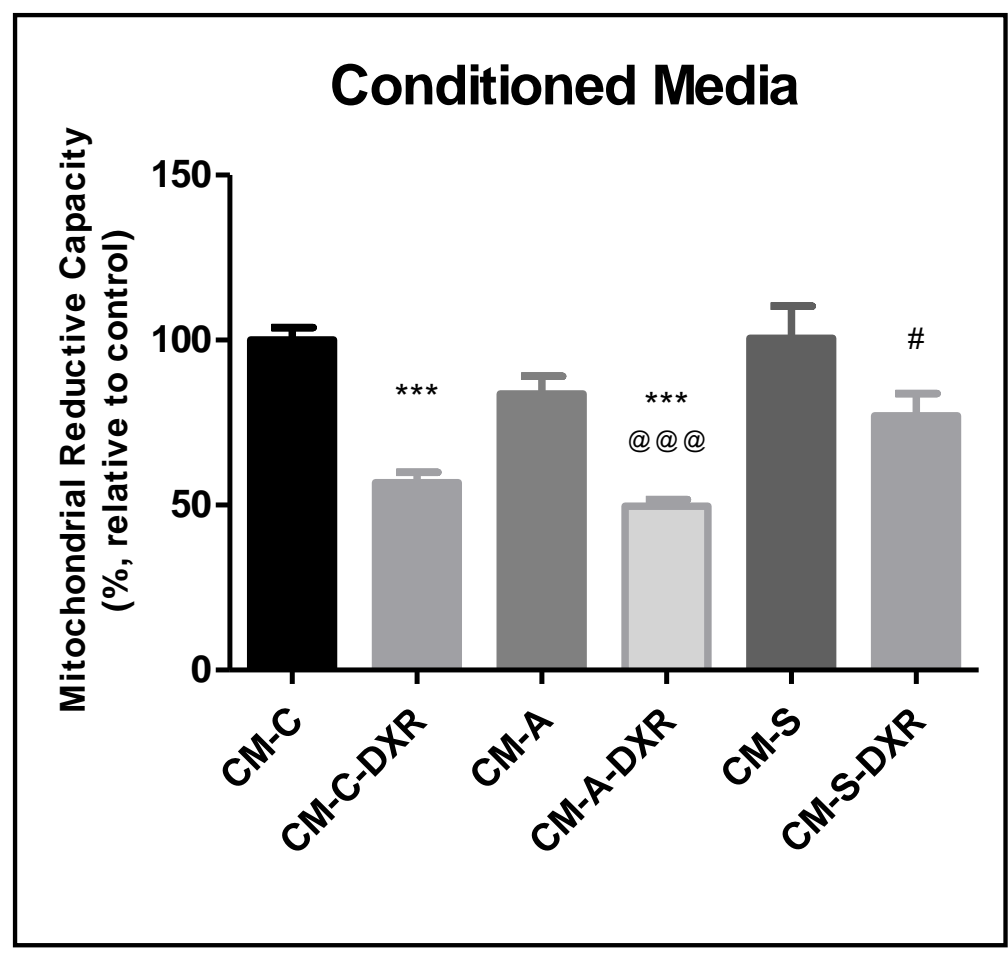

Figure 3.12: Percentage viability of E0771 cells after a conditioned media and DXR treatment period determined by an MTT assay. For apoptosis, MEFs were seeded and treated with DXR $(1 \mu \mathrm{M})$ for 24 hours, where after the $\mathrm{CM}$ was collected. E0771 cells were incubated with the collected $\mathrm{CM}$ while the CM-A-DXR group was also treated with DXR $(1 \mu \mathrm{M})$. For senescence, MEFs were treated with DXR $(2 \mu \mathrm{M})$ for 4 hours, where after the CM was collected during day 7, 10 and 13 . E0771 cells were incubated in the collected CM (CM-S), while the CM-S-DXR group was also treated with DXR $(1 \mu \mathrm{M})$. Results are presented as means \pm SEM $(n=3)$. The asterisk $\left({ }^{*}\right)$ denotes a significant decrease relative to the control $(p<0.001)$; the $(@)$ denotes a significant decrease relative to CM-A $(p<0.001)$ and $(\#)$ denotes a significant increase in CM-S-DXR relative to CM-C-DXR and CM-A-DXR $(p<0.05)$.

\subsubsection{Apoptosis activation in E0771 cells following Conditioned media and DXR treatment}

\subsubsection{Caspase 8 cleavage to determine apoptosis in E0771 cells after Conditioned media and DXR treatment}

Caspase 8 is involved in the intrinsic apoptosis pathway and membranes were therefore probed with caspase 8 to determine if the apoptotic pathway was activated after the 24-hour treatment period, by determining the amount of cleaved caspase 8 expression. Cleaved caspase 8 expression was significantly decreased in the senescence CM treated with DXR (CM-S-DXR) when compared to the control group treated with DXR (CM-C-DXR) $(p<0.05)$. No significant changes in cleaved caspase 8 were seen in the apoptosis CM $(p>0.05)$. 

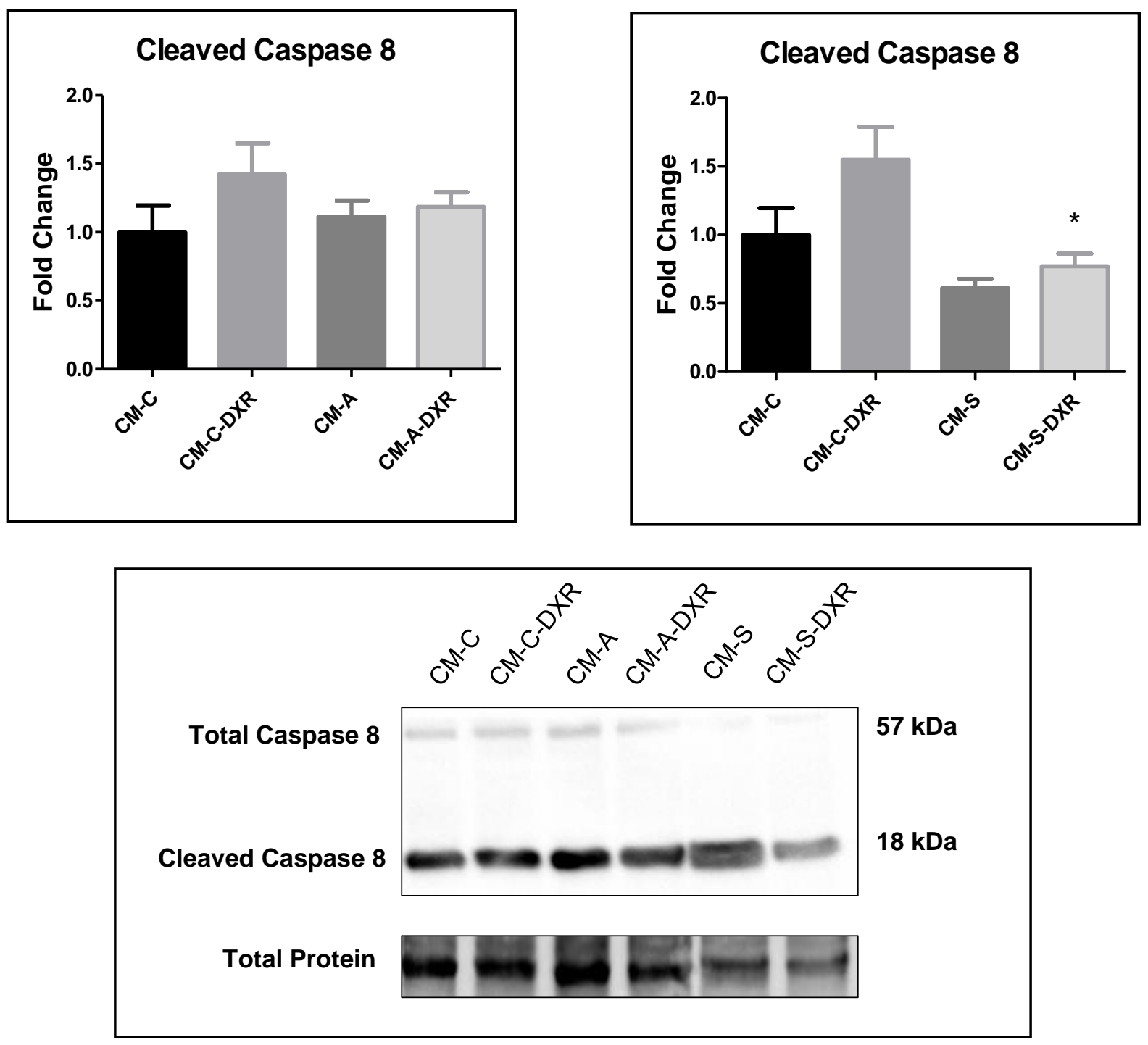

Figure 3.13: Western blot analysis to detect Caspase 8 cleavage in E0771 cells. E0771 cells were seeded and treated with either control, apoptosis or senescence Conditioned media collected from MEFs as described earlier or treated with $\mathrm{CM}$ and $1 \mu \mathrm{M}$ DXR for 24 hours. Proteins $(50 \mu \mathrm{g})$ from E0771 cells were separated by SDS-PAGE and transferred to a PVDF membrane for western blot analysis. Caspase 8 expression and cleaved caspase 8 is shown for control CM (lane 1), control CM and DXR (lane 2), apoptosis CM (lane 3), apoptosis CM and DXR (lane 4), senescence CM (lane 5) and senescence CM and DXR (lane 6). Results are presented as means \pm SEM $(n=3)$. For cleaved caspase 8 , the asterisk $\left(^{*}\right)$ denotes a significant decrease in CM-S-DXR when compared to CM-C-DXR $(p<0.05)$. 


\subsubsection{Caspase 9 cleavage to determine apoptosis in E0771 cells after Conditioned media and DXR treatment}

Membranes were incubated in caspase 9 primary antibody to determine whether the intrinsic or extrinsic apoptosis pathway is involved in E0771 cell death after the treatment period. However, no significant changes were observed in caspase 9 expression ( $p>0.05$ ).
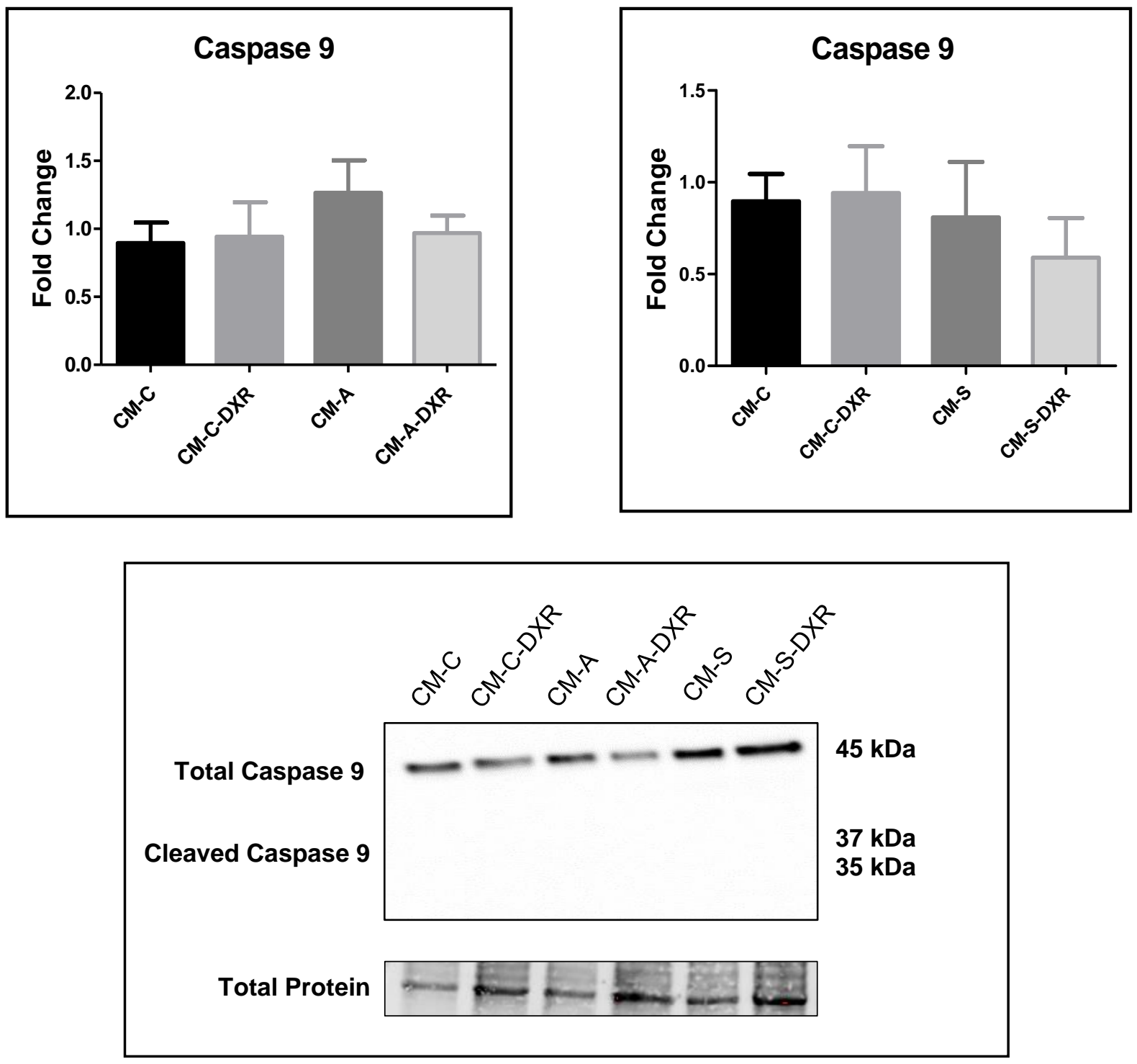

Figure 3.14: Western blot analysis to detect Caspase 9 cleavage in E0771 cells. E0771 cells were seeded and treated with either control, apoptosis or senescence conditioned media collected from MEFs as described earlier or treated with $\mathrm{CM}$ and $1 \mu \mathrm{M}$ DXR for 24 hours. Proteins $(50 \mu \mathrm{g})$ from E0771 cells were separated by SDS-PAGE and transferred to a PVDF membrane for western blot analysis. caspase 9 expression and cleaved caspase 8 is shown for control CM (lane 1), control CM and DXR (lane 2), apoptosis CM (lane 3), apoptosis CM and DXR (lane 4), senescence CM (lane 5) and senescence CM and DXR (lane 6). Results are presented as means \pm SEM ( $n=3)$. No significant data was observed $(p>0.05)$. 


\subsubsection{Caspase 3 to determine apoptosis in E0771 cells after Conditioned media and DXR treatment}

Caspase 3 is a frequently activated death protease, which catalyses the cleavage of many key cellular proteins and interacts with caspase 8 and caspase 9 during programmed cell death. Membranes were therefore probed with caspase 3 to determine if apoptosis was activated downstream. Furthermore, after the 24-hour treatment period with CM and DXR, senescence CM with DXR (CM-S-DXR) was significantly decreased when compared to the control DXR group (CM-C-DXR) $(p<0.05)$. No significant changes were seen in the apoptosis CM groups ( $p>0.05)$.
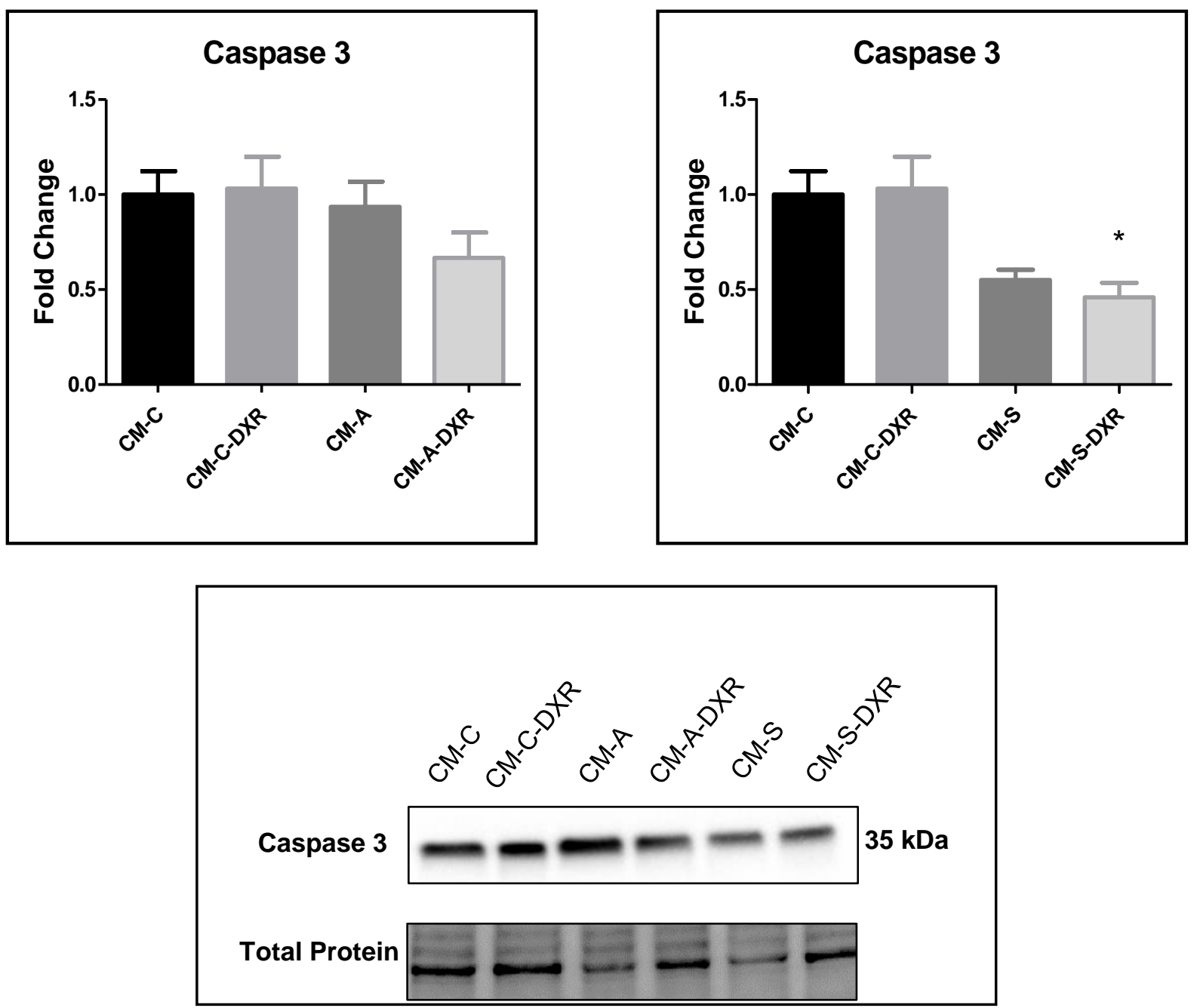

Figure 3.15: Western blot analysis to detect Caspase 3 in E0771 cells. E0771 cells were seeded and treated with either control, apoptosis or senescence conditioned media collected from MEFs as described earlier or treated with CM and $1 \mu \mathrm{M}$ DXR for 24 hours. Proteins $(50 \mu \mathrm{g})$ from E0771 cells were separated by SDS-PAGE and transferred to a PVDF membrane for western blot analysis. caspase 3 expression is shown for control CM (lane 1), control CM and DXR (lane 2), apoptosis CM (lane 3), apoptosis CM and DXR (lane 4), senescence CM (lane 5) and senescence CM and DXR (lane 6). 
Results are presented as means $\pm \operatorname{SEM}(n=3)$. The asterisk $\left(^{*}\right)$ denotes a significant decrease in caspase 3 expression in CM-S-DXR relative to CM-C-DXR $(p<0.05)$.

\subsubsection{Poly (ADP-ribose) polymerase cleavage to confirm apoptosis induction in E0771 cells after conditioned media and DXR treatment}

PARP is involved in programmed cell death and membranes were therefore probed for PARP to determine if apoptosis was induced in the E0771 cells after the 24-hour treatment period. Total PARP expression significantly decreased in the senescence CM (CM-S) group relative to the control CM $(C M-C)(p<0.05)$. There was also a significant decrease in total PARP expression in the senescence CM treated with DXR (CM-S-DXR) when compared to control CM treated with DXR (CM-C-DXR) $(p<0.05)$. However, no PARP cleavage was observed.
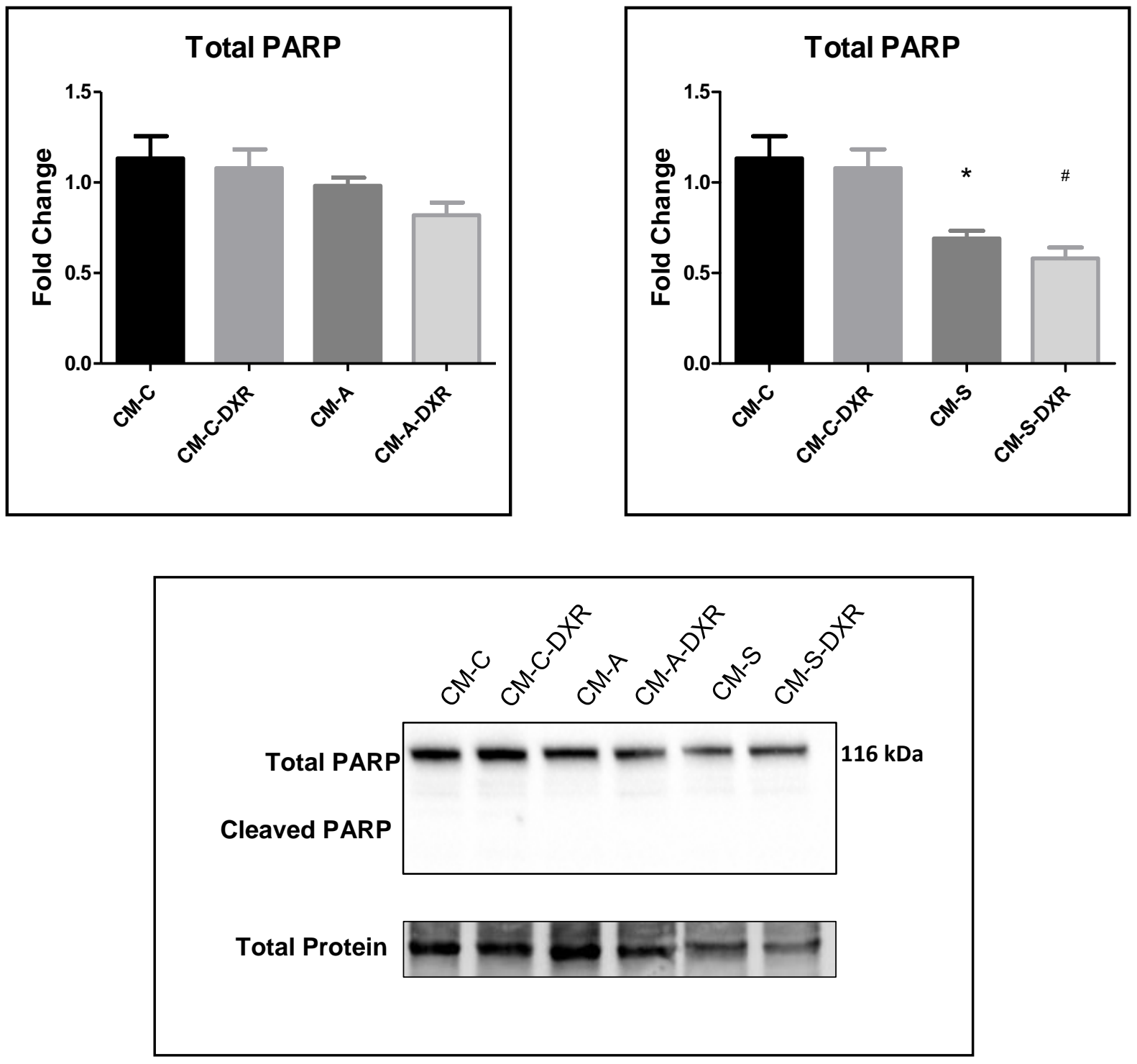
Figure 3.16: Western blot analysis to detect PARP cleavage in E0771 cells. E0771 cells were seeded and treated with either control, apoptosis or senescence conditioned media collected from MEFs or treated with CM and $1 \mu \mathrm{M}$ DXR for 24 hours. Proteins $(50 \mu \mathrm{g})$ from E0771 cells were separated by SDS-PAGE and transferred to a PVDF membrane for western blot analysis. PARP expression is shown for control CM (lane 1), control CM and DXR (lane 2), apoptosis CM (lane 3), apoptosis CM and DXR (lane 4), senescence CM (lane 5) and senescence CM and DXR (lane 6). Results are presented as means $\pm \operatorname{SEM}(n=3)$. The asterisk $\left({ }^{*}\right)$ denotes a significant decrease in total PARP expression in CM-S relative to the control $(C M-C)(p<0.05)$ and $(\#)$ denotes a significant decrease in total PARP expression in CM-S-DXR when compared to CM-C-DXR $(p<0.05)$.

\subsubsection{Senescence determination in E0771 cells after conditioned media and DXR treatment}

\subsubsection{Mini chromosome Maintenance Complex Component 2 (MCM2) expression in E0771 cells following treatment}

MCM2 plays an important role in the cell cycle regulating DNA replication and the onset of cell division. MCM2 expression significantly increased in senescence CM (CM-S) when compared to the control $(C M-C)(p<0.05)$. The expression of MCM2 also increased significantly in the E0771 cells when treated with senescence CM and $1 \mu \mathrm{M}$ DXR (CM-S-DXR), when compared to the control CM (CM-C) as well as the control CM treated with $1 \mu \mathrm{M}$ DXR (CM-C and CM-C$D X R)(p<0.01)$. No significant changes were observed in apoptosis CM relative to the control $\mathrm{CM}(\mathrm{p}>0.05)$.
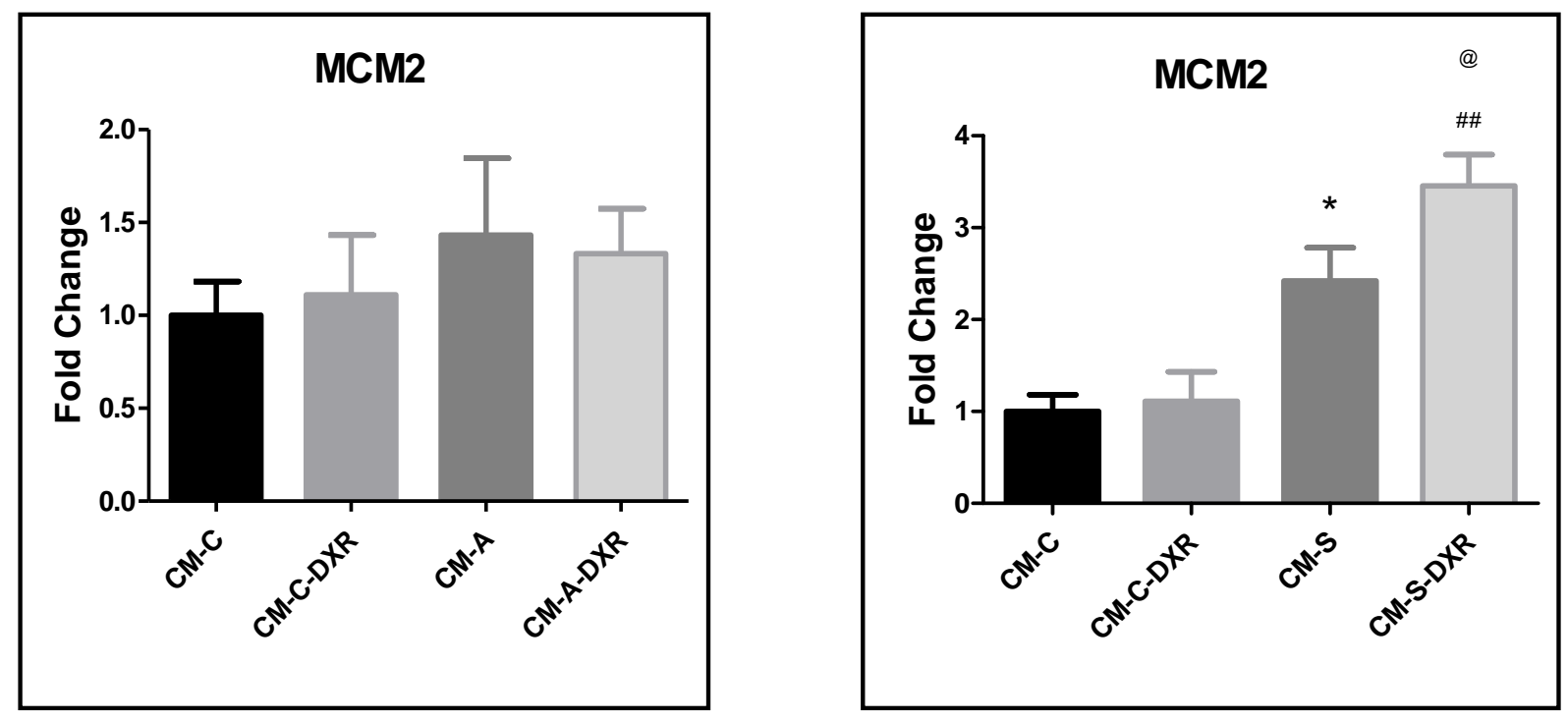


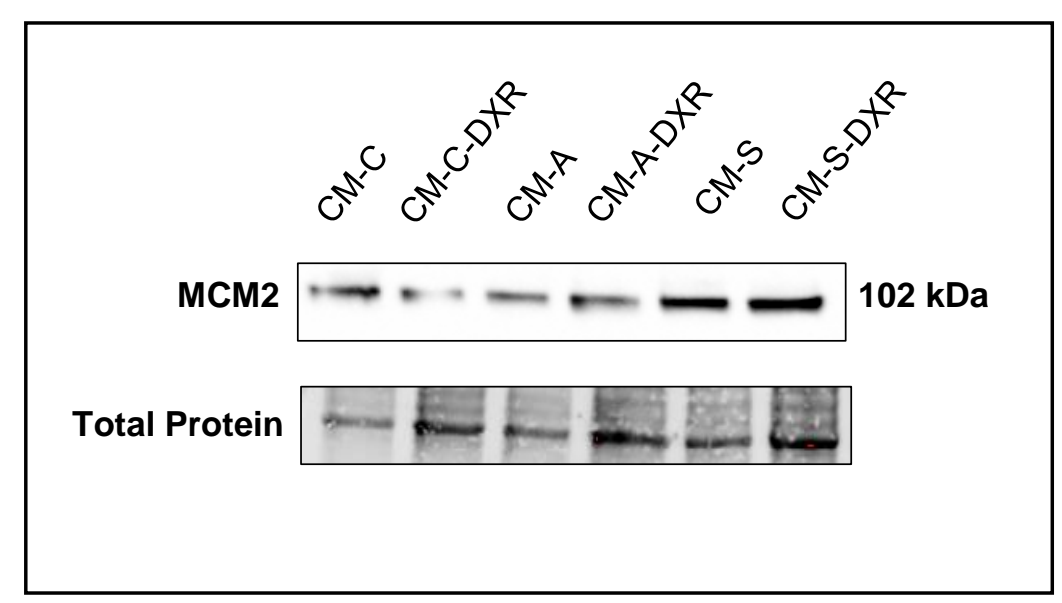

Figure 3.17: Western blot analysis to detect MCM2 in E0771 cells. E0771 cells were seeded and treated with either control, apoptosis or senescence conditioned media collected from MEFs as described earlier or treated with $\mathrm{CM}$ and $1 \mu \mathrm{M}$ DXR for 24 hours. Proteins $(50 \mu \mathrm{g})$ from E0771 cells were separated by SDS-PAGE and transferred to a PVDF membrane for western blot analysis. MCM2 expression is shown for control CM (lane 1), control CM and DXR (lane 2), apoptosis CM (lane 3), apoptosis CM and DXR (lane 4), senescence CM (lane 5) and senescence CM and DXR (lane 6). Results are presented as means \pm SEM $(n=3)$. The asterisk $\left(^{*}\right)$ denotes a significant increase in MCM2 expression in CM-S-DXR relative to CM-C $(p<0.01)$; (\#) denotes a significant increase in MCM expression in CM-S relative to the control $(C M-C)(p<0.01)$ and $(@)$ denotes a significant increase in CM-S-DXR relative to CM-C-DXR $(p<0.05)$.

\subsubsection{2 p53 expression in E0771 cells following treatment}

p53 is a tumour suppressor protein and when activated, it induces apoptosis, senescence or cell cycle arrest in response to DNA damaging agents or abnormal oncogene transformation. The expression of p53 significantly increased in the apoptosis CM treated with DXR (CM-ADXR) when compared to the control CM treated with DXR (CM-C-DXR) $(p<0.05)$. No significant changes in p53 expression was obtained in the senescence CM relative to the control $(p>0.05)$. 

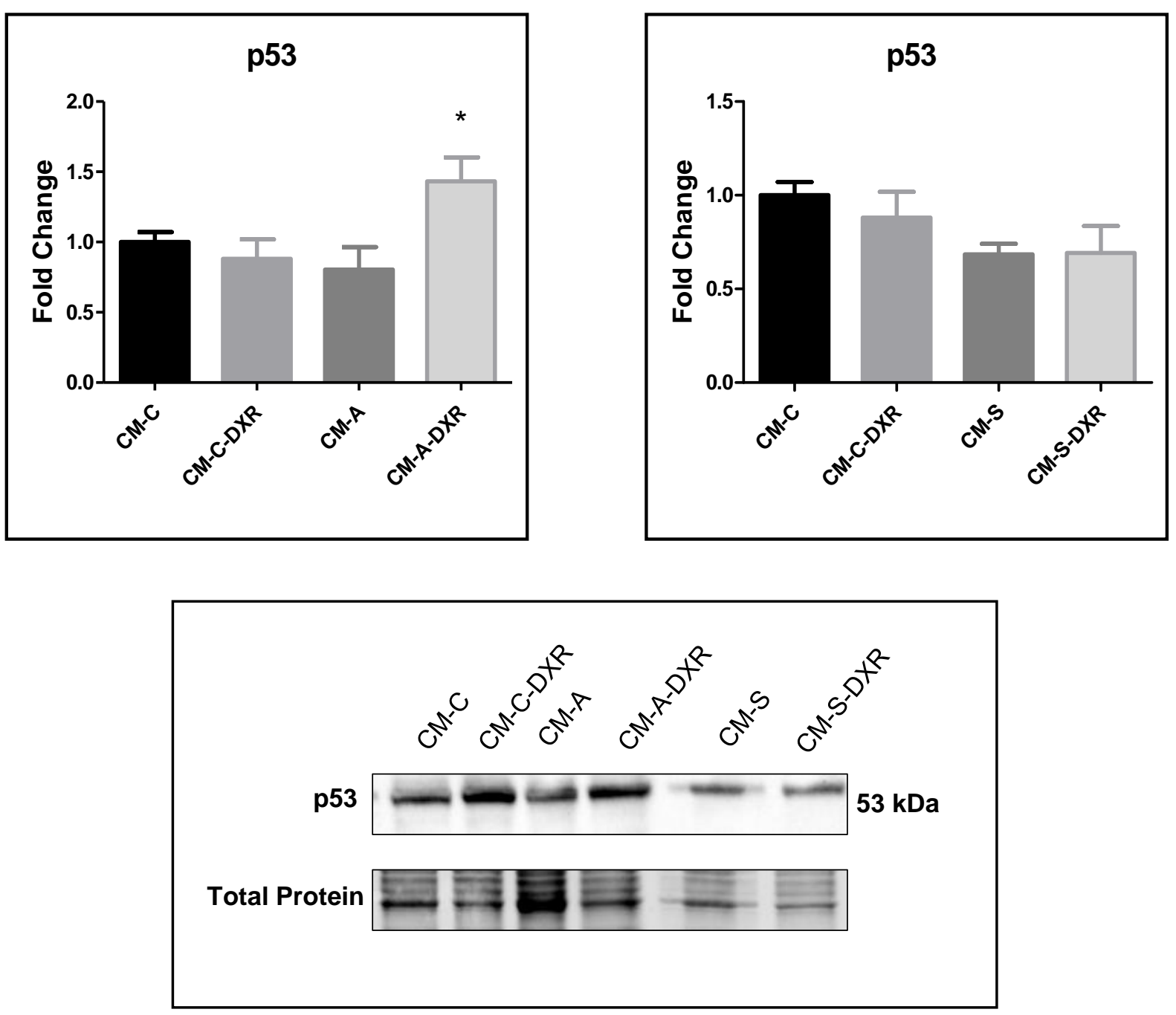

Figure 3.18: Western blot analysis to detect p53 in E0771 cells. E0771 cells were seeded and treated with either control, apoptosis or senescence conditioned media collected from MEFs as described earlier or treated with $\mathrm{CM}$ and $1 \mu \mathrm{M}$ DXR for 24 hours. Proteins $(50 \mu \mathrm{g})$ from E0771 cells were separated by SDS-PAGE and transferred to a PVDF membrane for western blot analysis. p53 expression is shown for control CM (lane 1), control CM and DXR (lane 2), apoptosis CM (lane 3), apoptosis CM and DXR (lane 4), senescence CM (lane 5) and senescence CM and DXR (lane 6). Results are presented as means $\pm \operatorname{SEM}(n=3)$. The asterisk $\left({ }^{*}\right)$ denotes a significant increase in p53 expression in CM-A-DXR when compared to the CM-C-DXR $(p<0.05)$.

\subsubsection{4 p16 expression in E0771 cells following treatment}

Expression of p16 maintains Retinoblastoma in a hypo phosphorylated state, which promotes the binding to E2F1 and leads to cell cycle arrest at the G1 phase (Serrano, 1997). The expression of p16 was significantly increased in the senescence CM treated with DXR (CM$S-D X R)$ relative to the control $(p<0.05)$. 

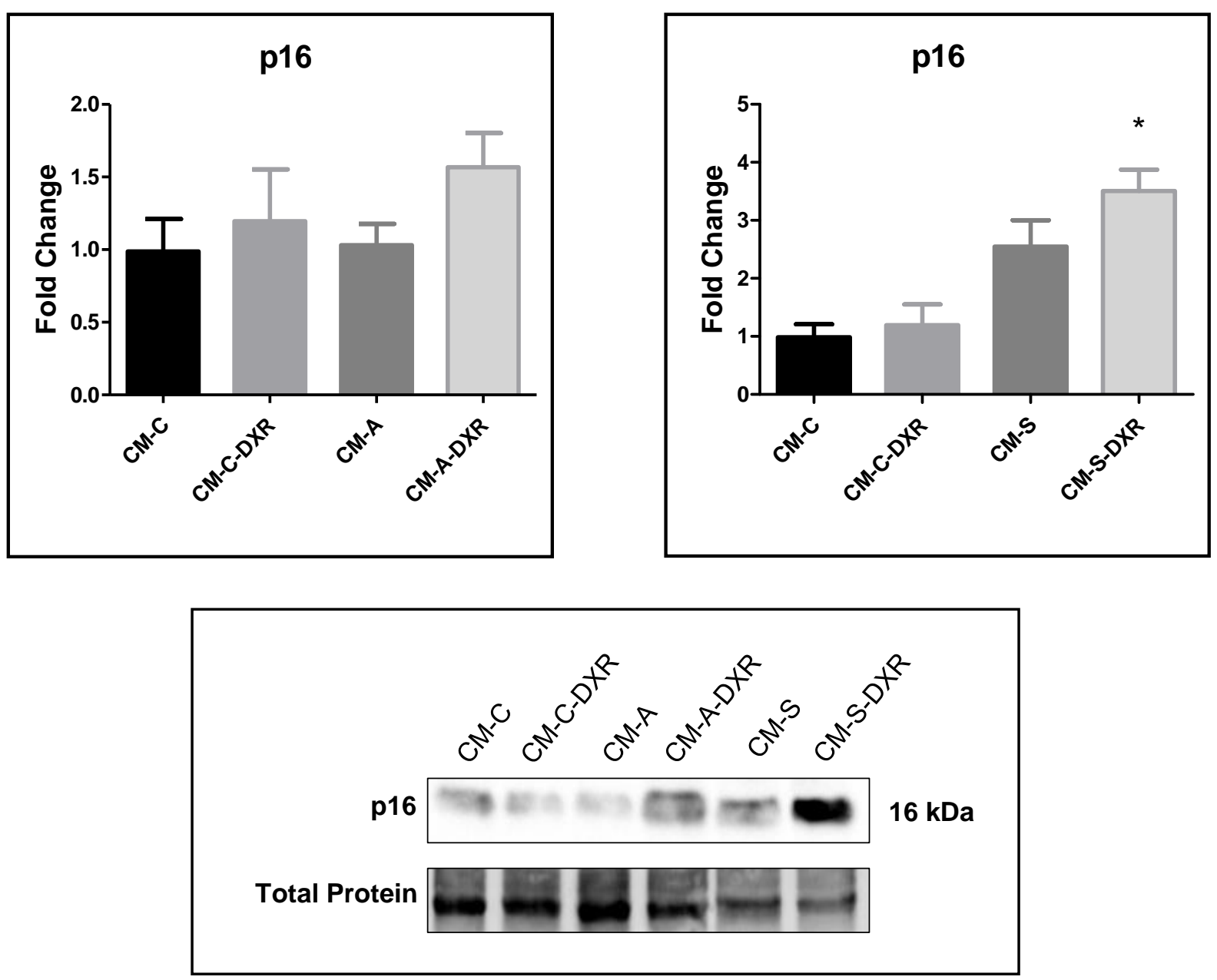

Figure 3.19: Western blot analysis to detect p16 in E0771 cells. E0771 cells were seeded and treated with either control, apoptosis or senescence conditioned media collected from MEFs as described earlier or treated with CM and $1 \mu \mathrm{M}$ DXR for 24 hours. Proteins $(50 \mu \mathrm{g})$ from E0771 cells were separated by SDS-PAGE and transferred to a PVDF membrane for western blot analysis. p16 expression is shown for control CM (lane 1), control CM and DXR (lane 2), apoptosis CM (lane 3), apoptosis CM and DXR (lane 4), senescence CM (lane 5) and senescence CM and DXR (lane 6). Results are presented as means $\pm \operatorname{SEM}(n=3)$. The asterisk $\left(^{*}\right)$ denotes a significant increase in $p 16$ expression in CM-S-DXR relative to the control $(C M-C)(p<0.05)$

\subsubsection{Retinoblastoma expression in E0771 cells following treatment}

Senescence induced by p16 acts through the RB pathway be inhibiting the function of CDKs. RB then prevents excessive cell growth by inhibiting cell cycle progression. RB expression was significantly increased in the senescence CM group treated with $1 \mu \mathrm{M}$ DXR when compared to all the other CM groups, including the CM groups treated with DXR $(p<0.05)$. 

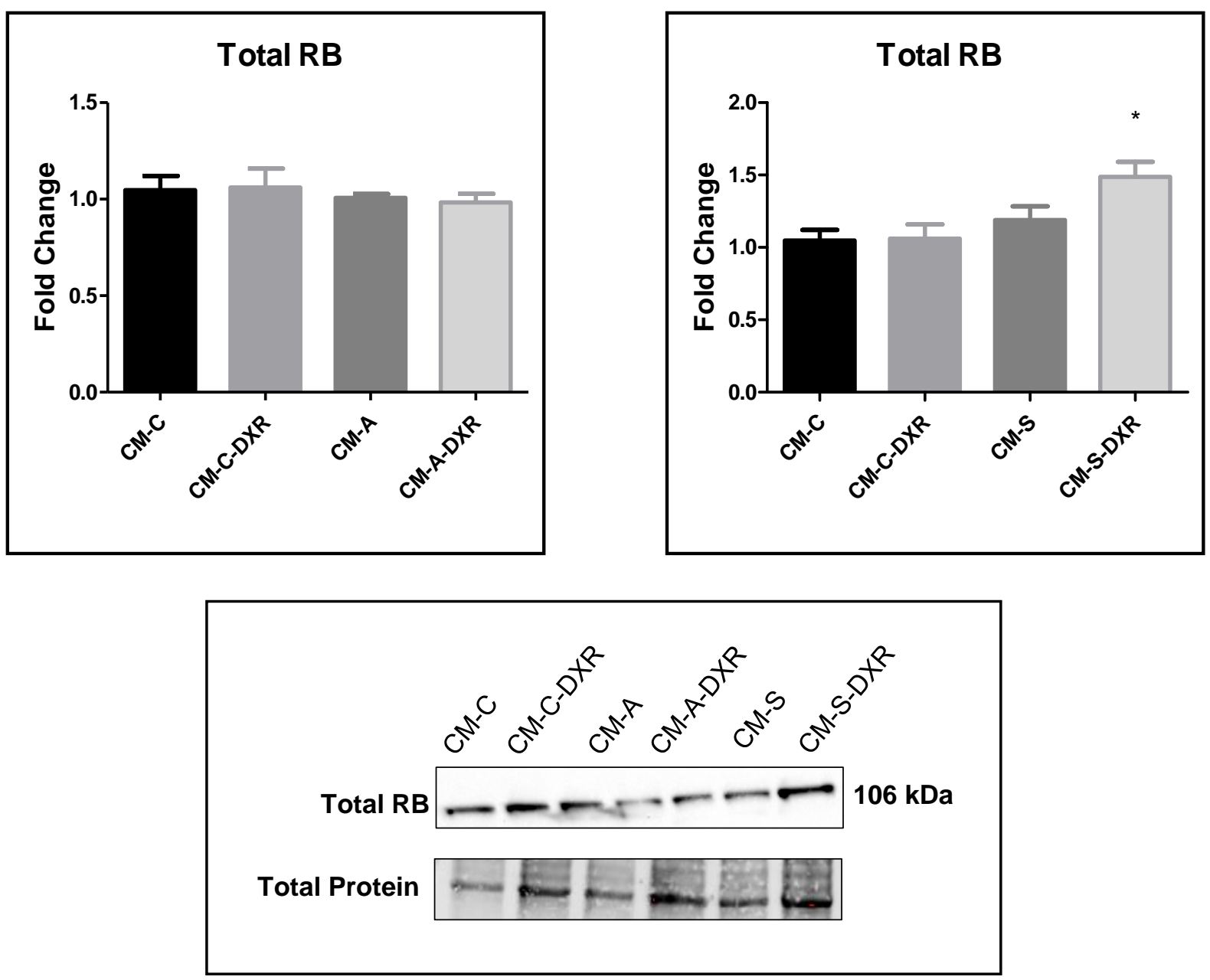

Figure 3.20: Western blot analysis to detect RB in E0771 cells. E0771 cells were seeded and treated with either control, apoptosis or senescence conditioned media collected from MEFs as described earlier or treated with $\mathrm{CM}$ and $1 \mu \mathrm{M}$ DXR for 24 hours. Proteins $(50 \mu \mathrm{g})$ from E0771 cells were separated by SDS-PAGE and transferred to a PVDF membrane for western blot analysis. RB expression is shown for control CM (lane 1), control CM and DXR (lane 2), apoptosis CM (lane 3), apoptosis CM and DXR (lane 4), senescence CM (lane 5) and senescence CM and DXR (lane 6). Results are presented as means \pm SEM $(n=3)$. The asterisk $\left({ }^{*}\right)$ denotes a significant increase in RB expression when compared to the CM-C $(p<0.05)$.

\subsubsection{Changes in cell viability following conditioned media and DXR treatment with autophagy inhibition}

An MTT assay was used to indirectly evaluate cell death when E0771 cells are incubated in CM for 24 hours obtained from MEFs and autophagy is inhibited. Bafilomycin (400 nM) was used to inhibit autophagy four hours prior to the end of the 24-hour treatment period. Cell viability significantly decreased when autophagy was inhibited (CM-C-Baf) relative to the control CM ( $p<0.05)$. A significant decrease in CM-C-DXR and CM-C-DXR-Baf could be seen 
relative to the control $(p<0.001)$. Once autophagy was inhibited in the CM-C-DXR group, cell viability decreased significantly $(p<0.05)$ after 24 hours, which differs from the significant increase in cell viability in both apoptosis (CM-A-DXR-BAF vs. CM-A-DXR) and senescence (CM-S-DXR-BAF vs. CM-S-DXR) conditioned media treated with $1 \mu \mathrm{M}$ DXR $(\mathrm{p}<0.01)$. Autophagy inhibition also significantly increased cell viability in the senescent CM (CM-S vs. CM-S-Baf) without the presence of DXR $(p<0.001)$. Furthermore, after the cells were treated with DXR, cell viability in the CM-S-DXR group was significantly higher than both the apoptosis $(C M-A-D X R)$ and control (CM-C-DXR) group $(p<0.05)$ (Figure 3.21).

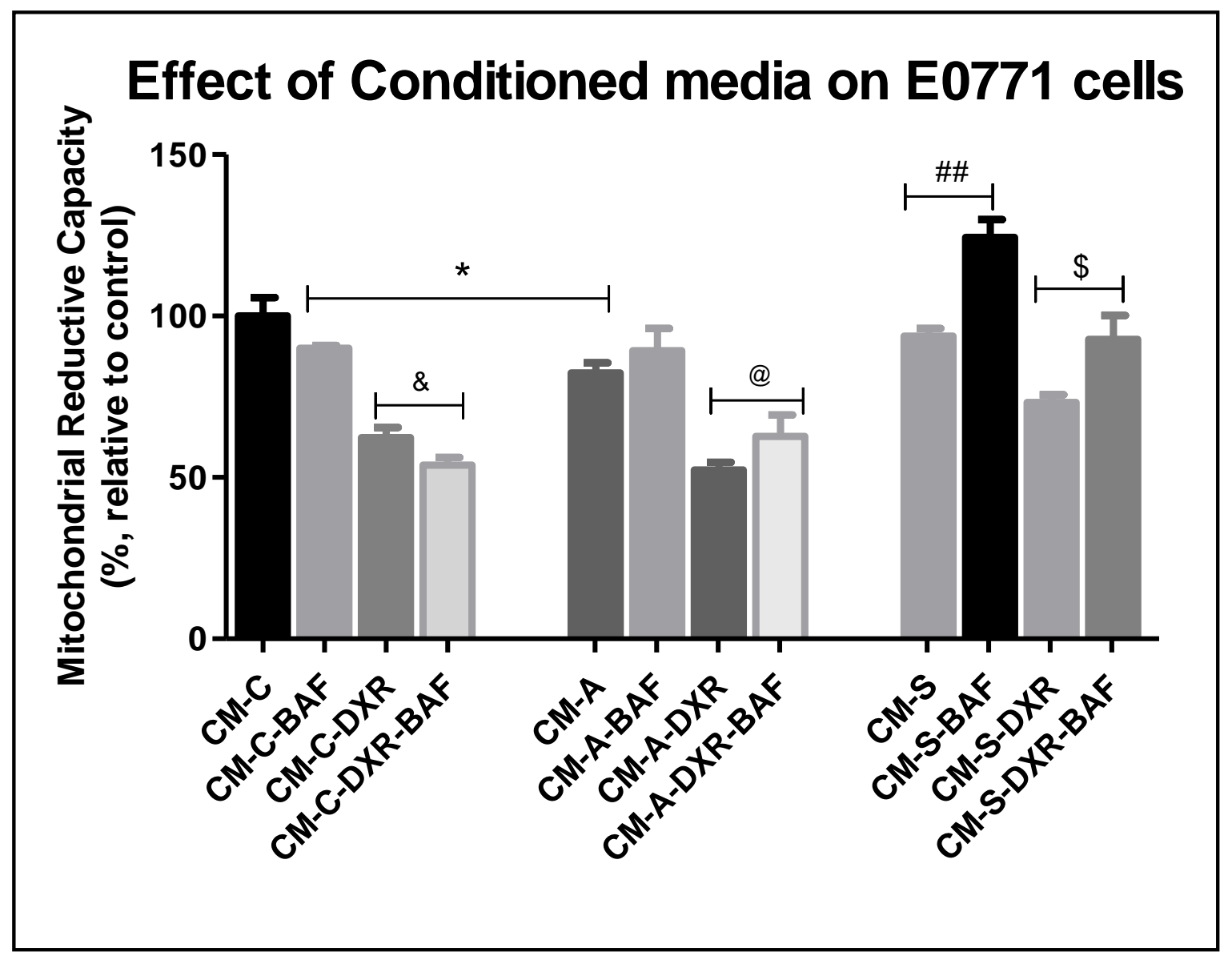

Figure 3.21: Percentage viability of E0771 cells after conditioned media and DXR treatment period with autophagy inhibition determined by an MTT assay. For apoptosis, MEFs were seeded and treated with DXR $(1 \mu \mathrm{M})$ for 24 hours, where after the CM was collected. E0771 cells were incubated with the collected CM while the CM-A-DXR group was also treated with DXR $(1 \mu \mathrm{M})$. For senescence, MEFs were treated with DXR $(2 \mu \mathrm{M})$ for 4 hours, where after the CM was collected during day 7, 10 and 13. E0771 cells were incubated in the collected CM (CM-S), while the CM-SDXR group was also treated with DXR $(1 \mu \mathrm{M})$. To inhibited autophagy, all the groups were treated with Bafilomycin $(400 \mathrm{nM})$ four hours prior to the end of the 24-hour treatment period respectively. 
Results are presented as means \pm SEM $(n=3)$. The asterisk (*) denotes a significant decrease relative to the control $(p<0.05)$; the $(\&)$ denotes a significant decrease in CM-C-DXR-Baf relative to CM-CDXR $(p<0.05)$; the $(@)$ denotes a significant increase in CM-A-DXR-Baf relative to CM-A-DXR $(p<0.05)$; (\#) denotes a significant increase in CM-S-Baf relative to CM-S $(p<0.01)$ and $(\$)$ denotes a significant increase in CM-S-DXR-Baf when compared to CM-C-DXR $(p<0.05)$.

\subsubsection{The expression of LC3 following conditioned media and DXR treatment with autophagy inhibition}

Microtubule-associated proteins $1 \mathrm{~A} / 1 \mathrm{~B}$ light chain 3B (LC3) is an essential protein involved in the autophagic pathway where it is involved in autophagosome biogenesis (Klionsky et al., 2016). The lipid modified form of LC3 (LC3 II) is involved in autophagosome formation and fusion events. The expression of LC3 II was significantly increased in the control CM treated with Bafilomycin (CM-C-Baf) relative to the control $(C M-C)(p<0.001)$. Furthermore, LC3 II was also increased in the apoptosis CM treated with Bafilomycin when compared to apoptosis CM $(\mathrm{p}<0.05)$. Similarly, the expression was also increased in senescence CM treated with Bafilomycin when compared to senescence CM (CM-S) $(p<0.001)$. However, no significant changes in autophagic flux were observed $(p>0.05)$.
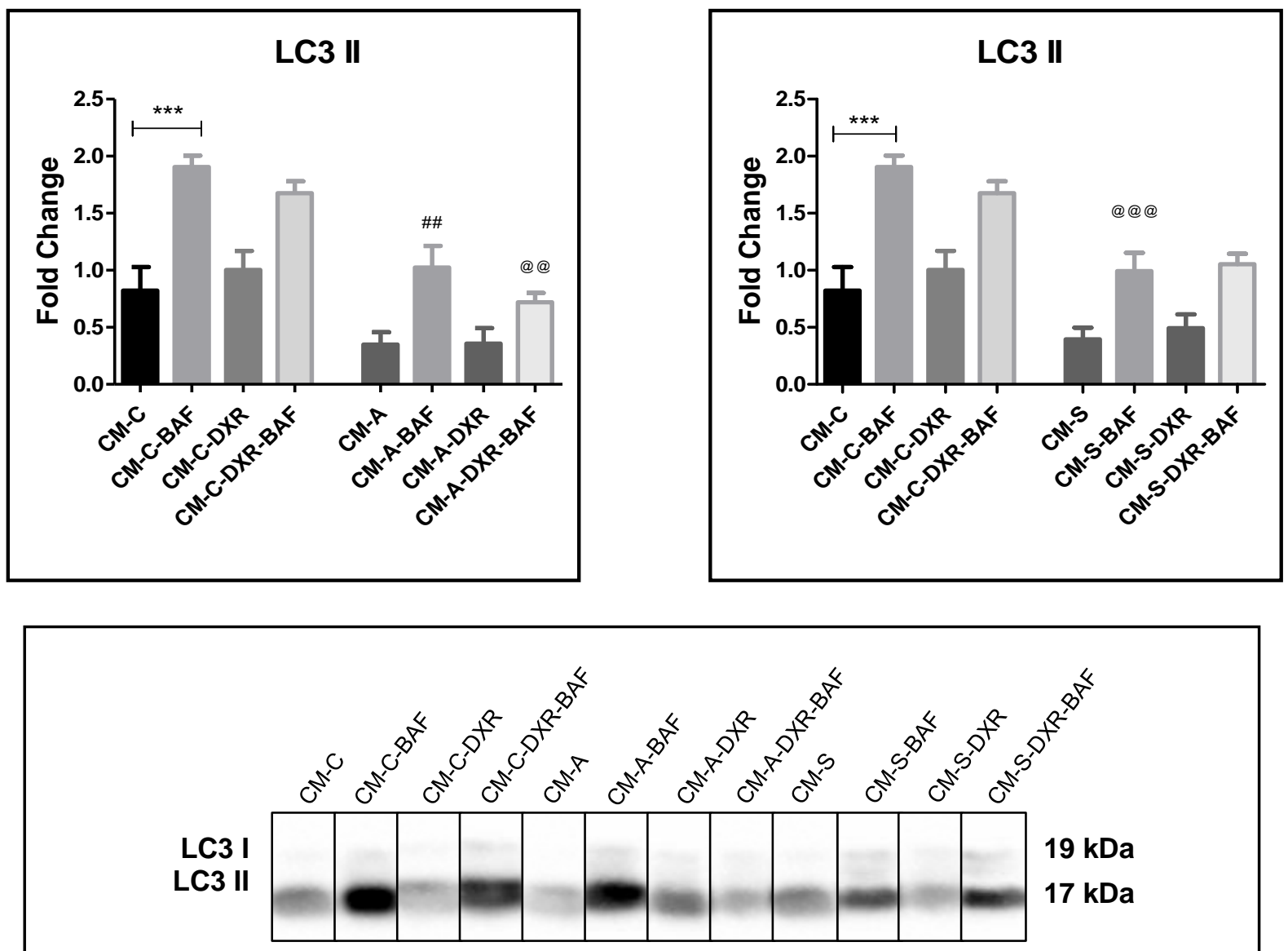

Total Protein

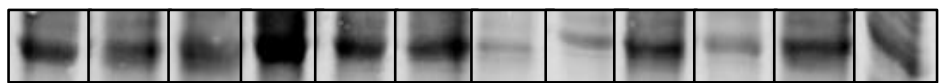


Figure 3.22: Western blot analysis to detect LC3 II in E0771 cells. E0771 cells were seeded and treated with either control, apoptosis or senescence conditioned media collected from MEFs as described earlier or treated with CM and $1 \mu \mathrm{M}$ DXR for 24 hours, alongside with Bafilomycin to inhibit autophagy. Proteins $(50 \mu \mathrm{g})$ from E0771 cells were separated by SDS-PAGE and transferred to a PVDF membrane for western blot analysis. LC3 II expression is shown for control CM (lane 1), control CM and Baf (lane 2), control CM and DXR (lane 3), control CM, DXR and Baf (lane 4), apoptosis CM (lane 5), apoptosis CM and Baf (lane 6), apoptosis and DXR (lane 7), apoptosis CM, DXR and Baf (lane 8), senescence CM (lane 9), senescence CM and Baf (lane 10), senescence CM and DXR (lane 11) and senescence CM, DXR and Baf (lane 12). Images were cropped to represent the different groups in the correct order. Results are presented as means \pm SEM $(n=3)$. For control and apoptosis CM the asterisk $\left({ }^{*}\right)$ denotes a significant decrease in LC3 II expression when compared to the control $(p<0.001)$. The (\#) denotes a significant decrease in CM-A-Baf relative to CM-C-Baf $(p<0.01)$ and (@) denotes a significant decrease in CM-A-DXR-Baf, relative to CM-C-DXR-Baf $(p<0.01)$. For control and senescence $\mathrm{CM}$ the asterisk $\left({ }^{*}\right)$ denotes a significant increase in LC3 II expression in Cm-C-Baf relative to the CM-C $(p<0.001)$ and $(@)$ denotes a significant decrease in CM-S-Baf relative to CM-C-Baf $(p<0.001)$.

\subsubsection{The expression of p62 following conditioned media and DXR treatment with autophagy inhibition}

The p62 protein, also referred to as sequestome 1 (SQSTM1), is a ubiquitin-binding scaffold protein which recognizes cellular toxic waste to be removed by autophagy (Rusten et al., 2010). The expression of p62 was significantly increased in control CM treated with Bafilomycin when compared to the control CM $(p<0.05)$. p62 expression was also significantly increased in control CM treated with DXR and Bafilomycin when compared to control CM treated with DXR $(p<0.01)$. A significant increase was observed in apoptosis $C M$ treated with DXR and Bafilomycin when compared to apoptosis CM treated with DXR $(p<0.05)$. No significant changes in senescence CM was observed ( $p>0.05)$. 

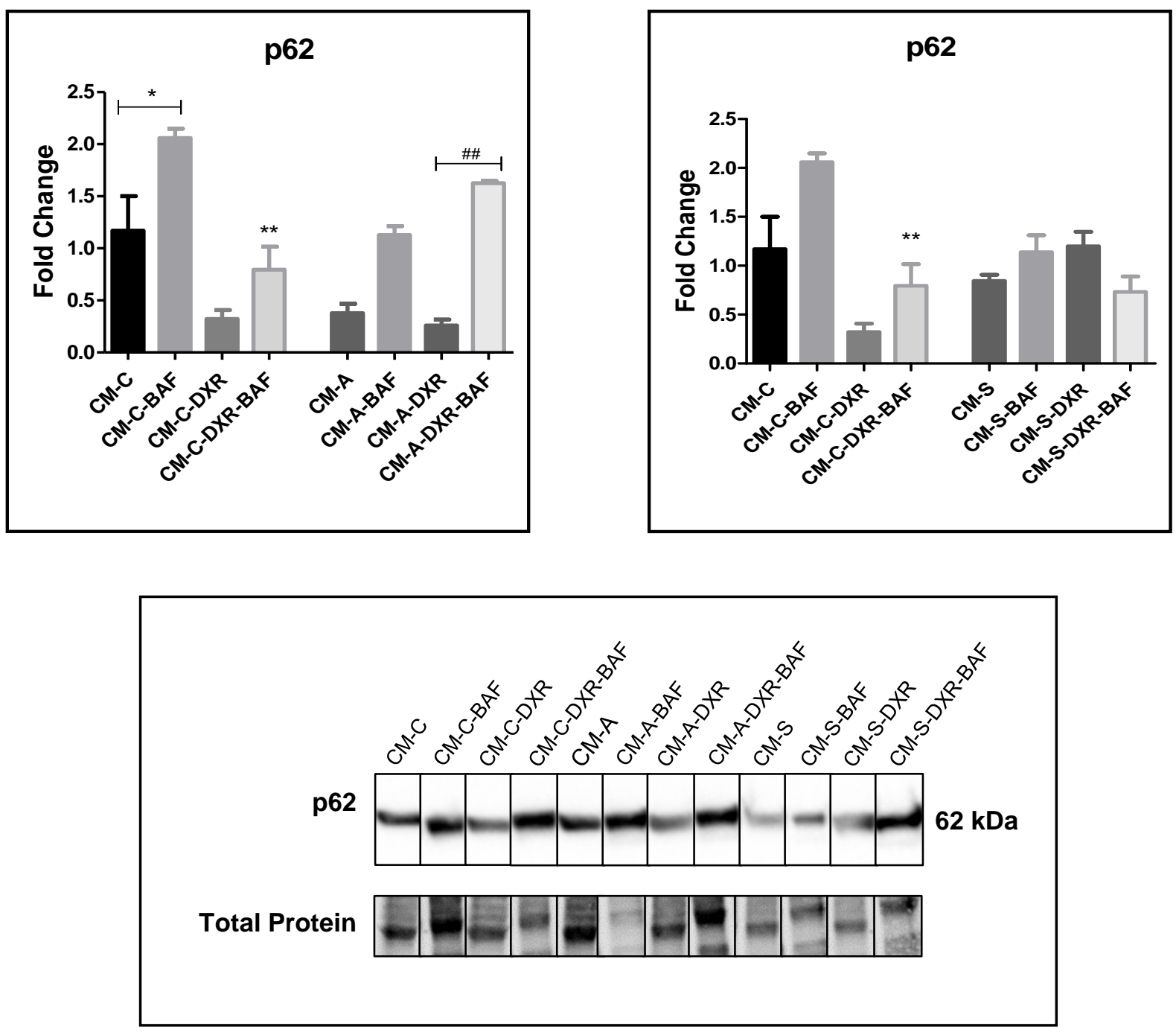

Figure 3.23: Western blot analysis to detect p62 in E0771 cells. E0771 cells were seeded and treated with either sontrol, apoptosis or senescence conditioned media collected from MEFs as described earlier or treated with CM and $1 \mu \mathrm{M}$ DXR for 24 hours, alongside with Bafilomycin to inhibit autophagy. Proteins $(50 \mu \mathrm{g})$ from E0771 cells were separated by SDS-PAGE and transferred to a PVDF membrane for western blot analysis. p62 expression is shown for control CM (lane 1), control CM and Baf (lane 2), control CM and DXR (lane 3), control CM, DXR and Baf (lane 4), apoptosis CM (lane 5), apoptosis CM and Baf (lane 6), apoptosis and DXR (lane 7), apoptosis CM, DXR and Baf (lane 8), senescence CM (lane 9), senescence CM and Baf (lane 10), senescence CM and DXR (lane 11) and senescence CM, DXR and Baf (lane 12). Images were cropped to represent the different groups in the correct order. Results are presented as means \pm SEM $(n=3)$. For control and apoptosis CM the asterisk $\left({ }^{*}\right)$ denotes a significant increase in p62 expression in CM-C-Baf when compared to the control $(p<0.05)$. The (\#) denotes a significant increase in CM-C-DXR-Baf relative to CM-C-DXR $(p<0.01)$. For control and senescence $\mathbf{C M}\left({ }^{*}\right)$ denotes a significant increase in p62 expression in CM-C-Baf relative to the control $\mathrm{CM}(\mathrm{p}<0.05)$. 


\subsubsection{The expression of phosphorylated Akt following conditioned media and DXR treatment with autophagy inhibition}

Akt, or also referred to as Protein kinase $B(P K B)$ is a serine/threonine specific protein kinase that is involved in many processes like apoptosis and cellular proliferation. Akt is located in the cytosol in its inactive conformation and translocates to the plasma membrane upon activation. The interaction between PIP3 and Akt causes a conformational change in Akt which exposes the Thr308 and Ser473 phosphorylation sites. Full phosphorylation of Ser473 is required for Akt activation (Miao et al., 2010). The expression of phosphorylated Akt (Ser473) (pAkt) was significantly increased in both CM-A (compared to CM-A-Baf) and CM-A-DXR (compared to CM-A-DXR-Baf) $(p<0.001)$. A significant increase was also observed in CM-S when compared to CM-S-Baf $(p<0.001)$. The expression of pAkt (Ser473) was significantly decreased in CM-S-DXR-Baf when compared to CM-S-DXR $(p<0.05)$. Furthermore, CM-C was significantly decreased when compared to CM-A and CM-S $(p<0.001)$ and CM-C-DXR was significantly decreased when compared to CM-A-DXR $(p<0.05)$.
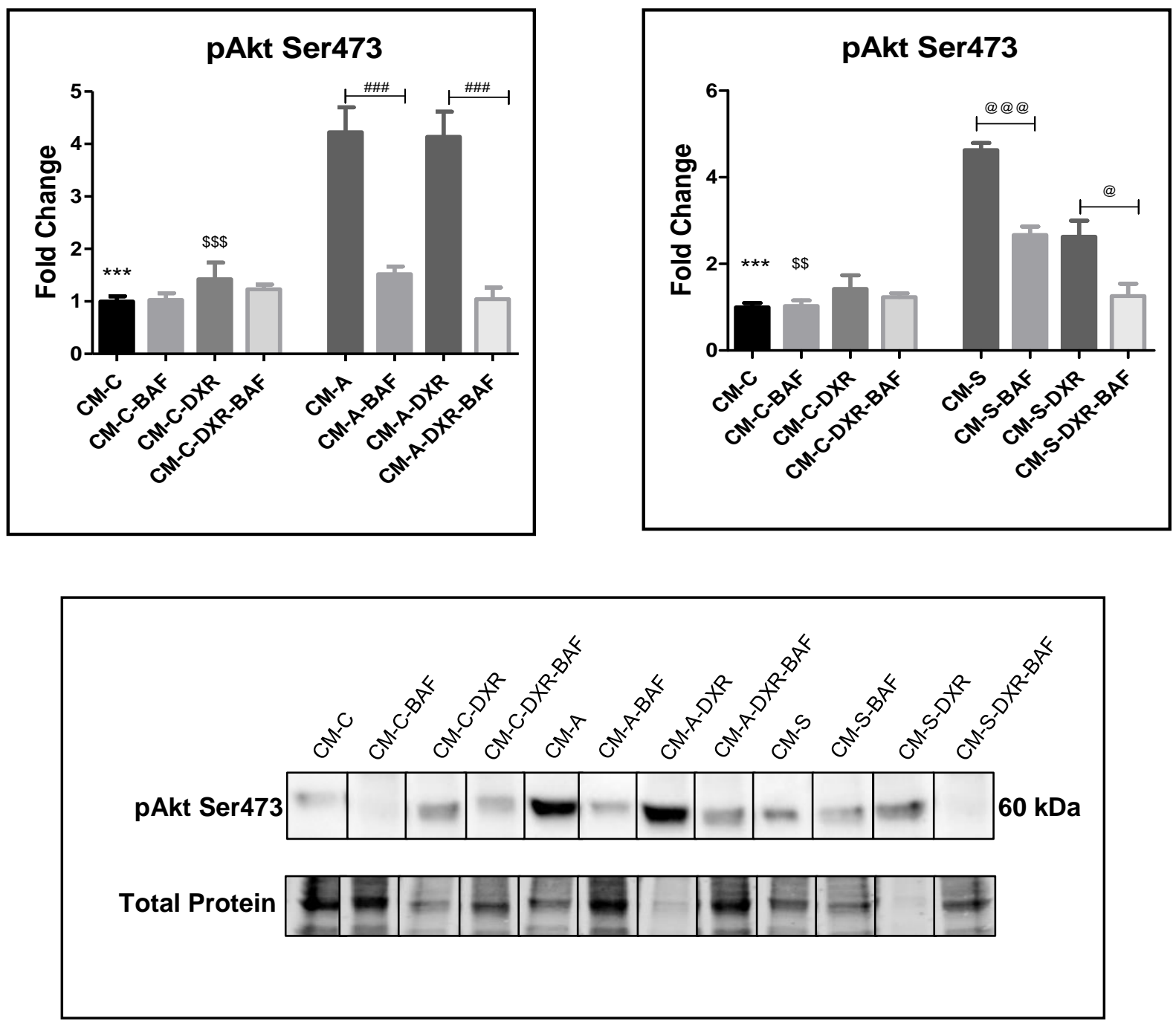
Figure 3.24.1: Western blot analysis to detect phosphorylated Akt in E0771 cells. E0771 cells were seeded and treated with either control, apoptosis or senescence conditioned media collected from MEFs as described earlier or treated with CM and $1 \mu \mathrm{M}$ DXR for 24 hours, alongside with Bafilomycin to inhibit autophagy. Proteins $(50 \mu \mathrm{g})$ from E0771 cells were separated by SDS-PAGE and transferred to a PVDF membrane for western blot analysis. pAkt expression is shown for control CM (lane 1), control CM and Baf (lane 2), control CM and DXR (lane 3), control CM, DXR and Baf (lane 4), apoptosis CM (lane 5), apoptosis CM and Baf (lane 6), apoptosis and DXR (lane 7), apoptosis CM, DXR and Baf (lane 8), senescence CM (lane 9), senescence CM and Baf (lane 10), senescence CM and DXR (lane 11) and senescence CM, DXR and Baf (lane 12). Images were cropped to represent the different groups in the correct order. Results are presented as means \pm SEM $(n=3)$. For control and apoptosis $C M$ the asterisk $\left(^{*}\right)$ denotes a significant decrease in $C M-C$ relative to $C M-A(p<0.001),(\$)$ denotes a significant decrease in CM-C-DXR relative to CM-A-DXR $(p<0.001)$ and the $(\#)$ denotes a significant increase in $C M-A$ and $C M-A-D X R$ relative to the Baf groups $(p<0.001)$. For control and senescence $C M(\$)$ denotes a significant decrease in CM-C-Baf relative to CM-S-Baf $(p<0.01)$ and (@) denotes a significant increase in CM-S $(p<0.001)$ and CM-S-DXR $(p<0.05)$ relative to the Baf groups.

\subsubsection{The expression of total Akt following conditioned media and DXR treatment with autophagy inhibition}

The expression of total Akt was significantly increased in control CM treated with DXR and Bafilomycin (CM-C-DXR-Baf) when compared to control CM treated with DXR (CM-C-DXR) $(p<0.01)$ and the expression was also increased in apoptosis $C M$ treated with $D X R$ and Bafilomycin (CM-A-DXR-Baf) when compared to apoptosis CM treated with DXR (CM-A-DXR) $(p<0.01)$. Senescence CM treated with DXR and Bafilomycin (CM-S-DXR-Baf) was also significantly increased when compared to CM-S-DXR $(p<0.05)$. Furthermore, Akt was significantly decreased in both CM-S and CM-A relative to control CM $(p<0.05)$.
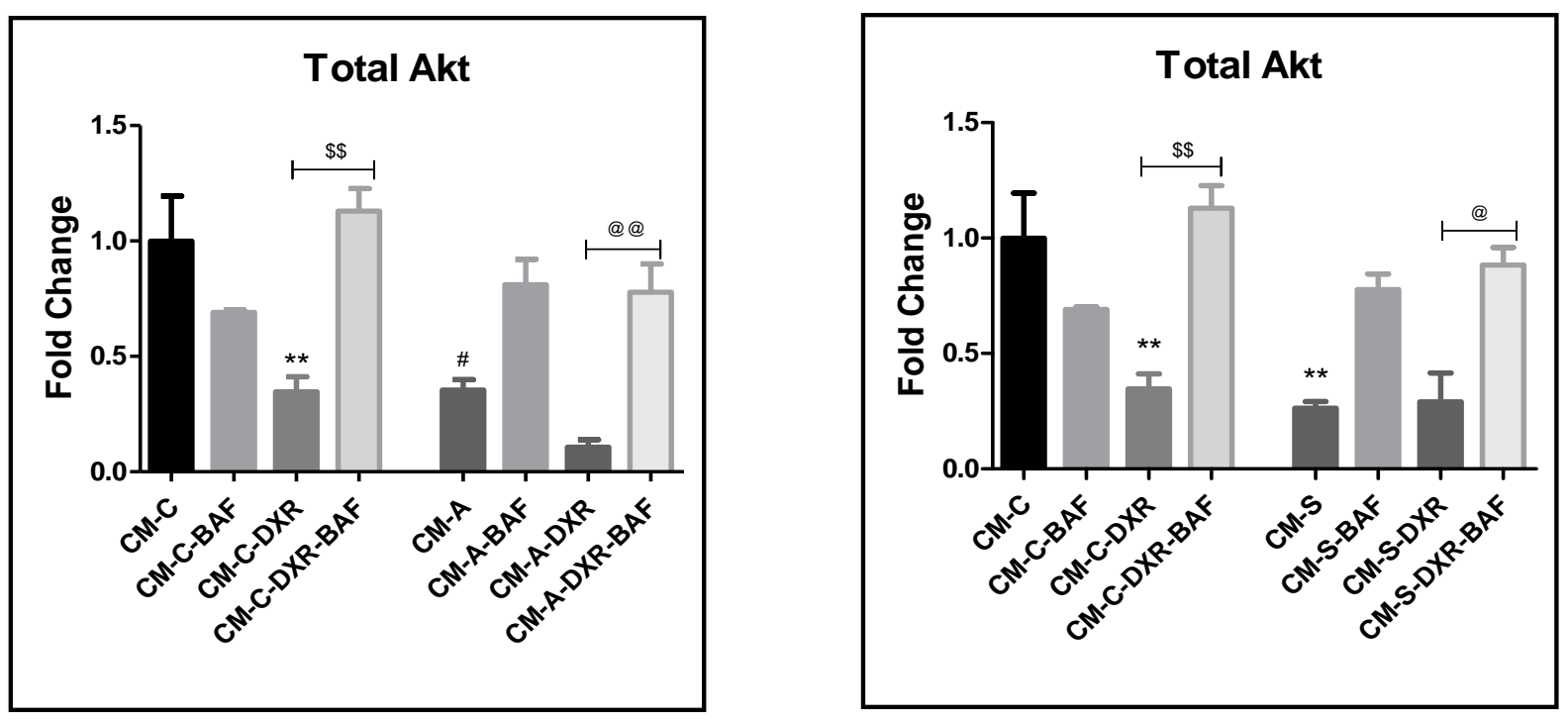


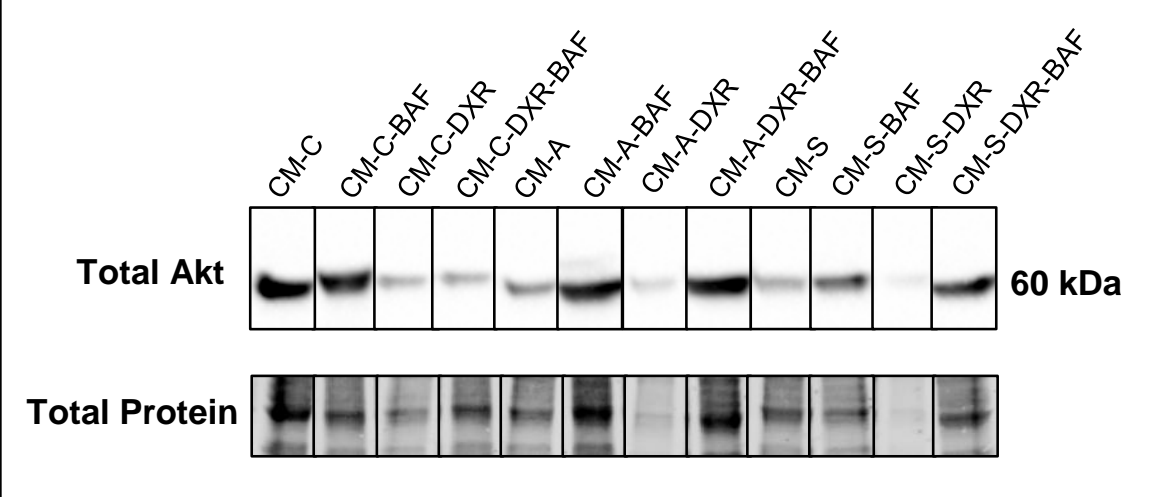

Figure 3.24.2: Western blot analysis to detect total Akt in E0771 cells. E0771 cells were seeded and treated with either control, apoptosis or senescence conditioned media collected from MEFs as described earlier or treated with $\mathrm{CM}$ and $1 \mu \mathrm{M}$ DXR for 24 hours, alongside with Bafilomycin to inhibit autophagy. Proteins $(50 \mu \mathrm{g})$ from E0771 cells were separated by SDS-PAGE and transferred to a PVDF membrane for western blot analysis. Total Akt expression is shown for control CM (lane 1), control CM and Baf (lane 2), control CM and DXR (lane 3), control CM, DXR and Baf (lane 4), apoptosis CM (lane 5), apoptosis CM and Baf (lane 6), apoptosis and DXR (lane 7), apoptosis CM, DXR and Baf (lane 8), senescence CM (lane 9), senescence CM and Baf (lane 10), senescence CM and DXR (lane 11) and senescence CM, DXR and Baf (lane 12). Images were cropped to represent the different groups in the correct order. Results are presented as means \pm SEM $(n=3)$. For control and apoptosis CM the asterisk $\left({ }^{*}\right)$ denotes a significant decrease in CM-C-DXR when compared to CM-C $(p<0.01)$ and $(\$)$ denotes a significant increase in CM-C-DXR-Baf when compared to CM-C-DXR $(p<0.01)$. The (\#) denotes a significant decrease in CM-A relative to CM-C $(p<0.05)$ and $(@)$ denotes a significant decrease in CM-A-DXR when compared to CM-A-DXR-Baf $(p<0.01)$. For control and senescence $\mathbf{C M}$ $\left({ }^{*}\right)$ denotes a significant decrease in CM-S and CM-C-DXR relative to CM-C $(p<0.01)$ and (@) denotes a significant increase in Akt in CM-S-DXR-Baf relative to CM-S-DXR $(p<0.05)$.

The ratio of phosphorylated Akt to total Akt was then calculated and used as a proliferative indicator. The expression of pAkt/Total Akt was significantly increased in apoptosis CM treated with DXR (CM-A-DXR) $(p<0.001)$ as well as in senescence CM $(C M-S)(p<0.001)$ and senescence CM treated with DXR (CM-S-DXR) $(p<0.05)$. Furthermore, the expression on pAkt/total Akt was also significantly increased in the apoptosis and senescence CM when compared to the control CM $(p<0.05)$. 

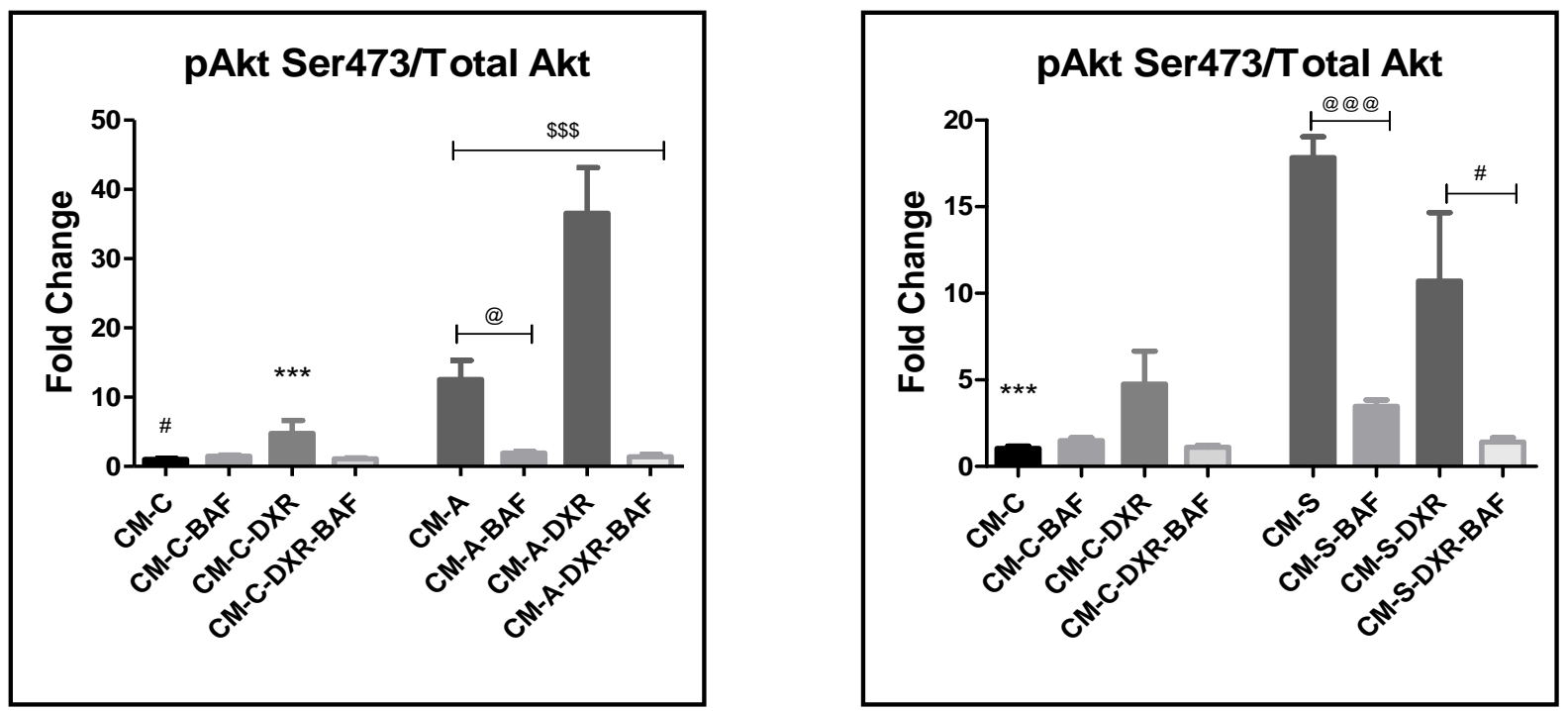

Figure 3.24.3: pAkt/total Akt in E0771 cells. The ratio between phosphorylated Akt and total Akt was determined. For control and apoptosis $\mathrm{CM}$ the asterisk $\left(^{*}\right)$ denotes a significant decrease in $\mathrm{CM}-\mathrm{C}$ DXR when compared to CM-A-DXR $(p<0.001)$, (\#) denotes a significant decrease in CM-C when compared to CM-A $(p<0.05)$. The (@) denotes a significant decrease in CM-A-Baf relative to CM-A $(p<0.05)$ and $(\$)$ denotes a significant increase in CM-A-DXR $(p<0.001)$. For control and senescence CM $\left({ }^{*}\right)$ denotes a significant decrease in CM-C when compared to CM-S $(p<0.001)$, (@) denotes a significant increase in CM-S relative to CM-S-Baf $(p<0.001)$ and $(\#)$ denotes a significant increase in CM-S-DXR relative to CM-S-DXR-Baf $(p<0.05)$.

\subsubsection{The expression of phosphorylated ERK following conditioned media and DXR treatment with autophagy inhibition}

The MAPK/ERK pathway (also referred to as the Ras-Raf-MEK-ERK pathway) transports signals from a cell surface receptor towards DNA in the cellular nucleus to activate processes like cell division, proliferation, differentiation and survival. Once ERK1/2 is phosphorylated by MEK1/2, it becomes activated and is translocated to the nucleus. Phosphorylated ERK1 (pERK 1) was significantly decreased in the control CM treated with Bafilomycin (CM-C-Baf) when compared to the control CM $(\mathrm{CM}-\mathrm{C})(\mathrm{p}<0.05)$. No significant changes in the apoptosis $C M$ were observed $(p<0.05)$. For senescence $C M$, $p E R K 1$ was significantly increased in senescence CM treated with DXR and Bafilomycin (CM-S-DXR-Baf) when compared to senescence CM treated with Bafilomycin (CM-S-Baf) as well as control CM treated with DXR and Bafilomycin (CM-C-DXR-Baf) $(\mathrm{p}<0.001)$. Additionally, control CM treated with Bafilomycin (CM-C-Baf) and control CM treated with DXR and Baf (CM-C-DXR-Baf) was significantly decreased relative to the control $(p<0.05)$. 
For phosphorylated ERK 2 (pERK 2), apoptosis CM treated with DXR (CM-A-DXR) was significantly increased when compared to apoptosis CM (CM-A), control CM treated with DXR (CM-C-DXR) as well as apoptosis CM treated with DXR and Bafilomycin (CM-A-DXR-Baf) $(p<0.001)$. The expression of $p E R K 2$ was also significantly increased in apoptosis CM treated with Bafilomycin (CM-A-Baf) when compared to the control treated with Bafilomycin (CM-CBaf) $(p<0.05)$. Additionally, pERK 2 was significantly increased in senescence CM treated with Bafilomycin (CM-S-Baf) when compared to senescence CM (CM-S) as well as the control groups $(p<0.001)$. The expression was also increased in senescence CM treated with DXR and Bafilomycin (CM-S-DXR-Baf) relative to senescence CM treated with DXR (CM-S-DXR) $(p<0.01)$.
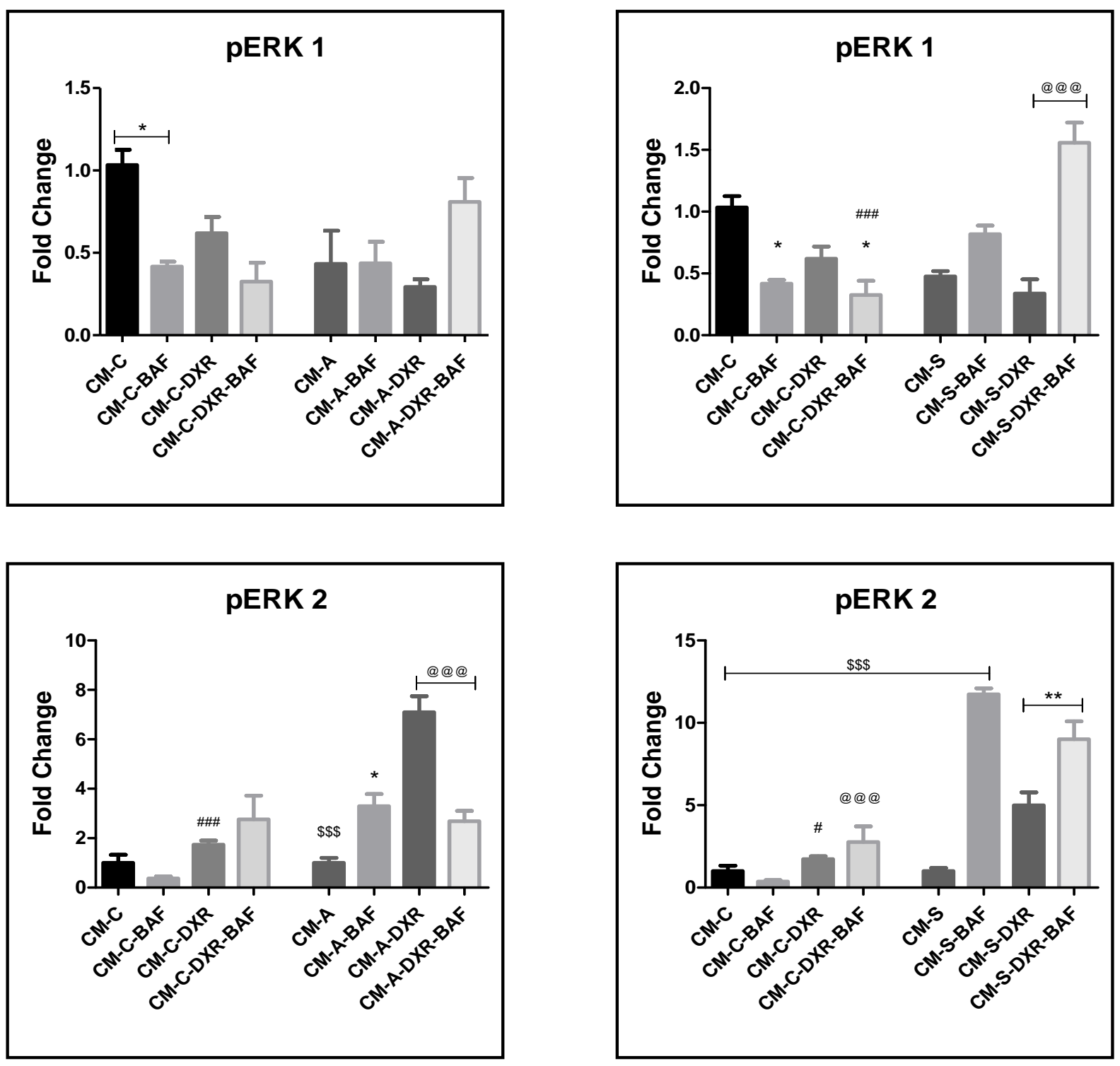


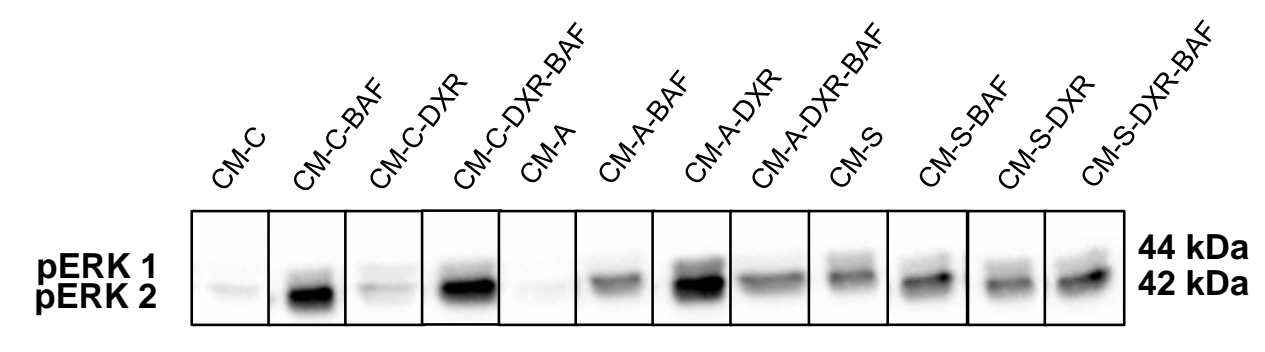

Total Protein

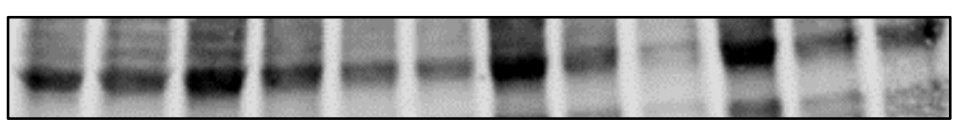

Figure 3.25.1: Western blot analysis to detect phosphorylated ERK in E0771 cells. E0771 cells were seeded and treated with either control, apoptosis or senescence conditioned media collected from MEFs as described earlier or treated with CM and $1 \mu \mathrm{M}$ DXR for 24 hours, alongside with Bafilomycin to inhibit autophagy. Proteins $(50 \mu \mathrm{g})$ from E0771 cells were separated by SDS-PAGE and transferred to a PVDF membrane for western blot analysis. Results are presented as means $\pm \operatorname{SEM}(n=3)$. pERK1/2 expression is shown for control CM (lane 1), control CM and Baf (lane 2), control CM and DXR (lane 3), control CM, DXR and Baf (lane 4), apoptosis CM (lane 5), apoptosis CM and Baf (lane 6), apoptosis and DXR (lane 7), apoptosis CM, DXR and Baf (lane 8), senescence CM (lane 9), senescence CM and Baf (lane 10), senescence CM and DXR (lane 11) and senescence CM, DXR and Baf (lane 12). pERK 1: For control and apoptosis CM the asterisk $\left(^{*}\right)$ denotes a significant decrease in CM-C-Baf when compared to $C M-C(p<0.05)$. For control and senescence $\mathbf{C M}\left(^{*}\right)$ denotes a significant decrease in CM-C-Baf and CM-C-DXR-Baf relative to the control $(p<0.05)$. The (\#) denotes a significant decrease in CM-C-DXR-Baf when compared to CM-S-DXR-Baf $(p<0.001)$ and (@) denotes a significant increase in CM-S-DXR-Baf relative to CM-S-DXR ( $<<0.001)$. pERK 2: For control and apoptosis CM the asterisk $\left(^{*}\right)$ denotes a significant increase in CM-A-Baf relative to CM-C-Baf $(p<0.05)$, (\#) denotes a significant decrease in CM-C-DXR relative to CM-A-DXR $(p<0.001)$ and $(@)$ denotes a significant increase in CM-A-DXR relative to CM-A-DXR-Baf $(p<0.001)$. For control and senescence CM (\#) denotes a significant decrease in CM-C-DXR relative to CM-S-DXR $(p<0.05)$, (@) denotes a significant decrease in CM-C-DXR-Baf relative to CM-S-DXR-Baf $(p<0.001)$; $(\$)$ denotes a significant increase in CM-S-Baf $(p<0.001)$ and $\left({ }^{*}\right)$ denotes a significant decrease in CM-S-DXR relative to CM-S-DXR-Baf $(p<0.01)$. 


\subsubsection{The expression of total ERK 1 and ERK 2 following conditioned media and DXR treatment with autophagy inhibition}

For apoptosis, total ERK 1 was significantly increased in CM-C-DXR-Baf when compared to all control CM groups $(p<0.001)$. The expression of total ERK1 was significantly increased in apoptosis CM (CM-A) when compared to control CM (CM-C) $(p<0.05)$ and was also increased in apoptosis CM treated with DXR and Bafilomycin (CM-A-DXR-Baf) when compared to apoptosis CM treated with DXR $(C M-A-D X R)(p<0.05)$. For senescence, total ERK 1 expression was significantly increased in senescence CM treated with Bafilomycin (CM-S-Baf) when compared to control CM treated with Baf $(C M-C-B a f)(p<0.001)$ and the expression was also increased in senescence CM treated with DXR and Bafilomycin (CM-S-DXR-Baf) when compared to senescence CM treated with DXR (CM-S-DXR) $(p<0.01)$.

For apoptosis, the expression of total ERK 2 was significantly increased in control CM treated with DXR and Bafilomycin (CM-C-DXR-Baf) relative to the control $(p<0.01)$. It was also increased in apoptosis CM treated with Bafilomycin (CM-A-Baf) when compared to apoptosis CM $(C M-A)(p<0.05)$ and was also increased in CM-A-Baf relative to the control treated with Bafilomycin (CM-C-Baf) $(\mathrm{p}<0.05)$. For senescence, CM treated with Bafilomycin (CM-S-Baf) was significantly increased when compared to CM-S as well as control CM treated with Bafilomycin $(p<0.01)$. The expression of total ERK 2 was also increased in senescence $C M$ treated with DXR (CM-S-DXR) $(p<0.05)$.
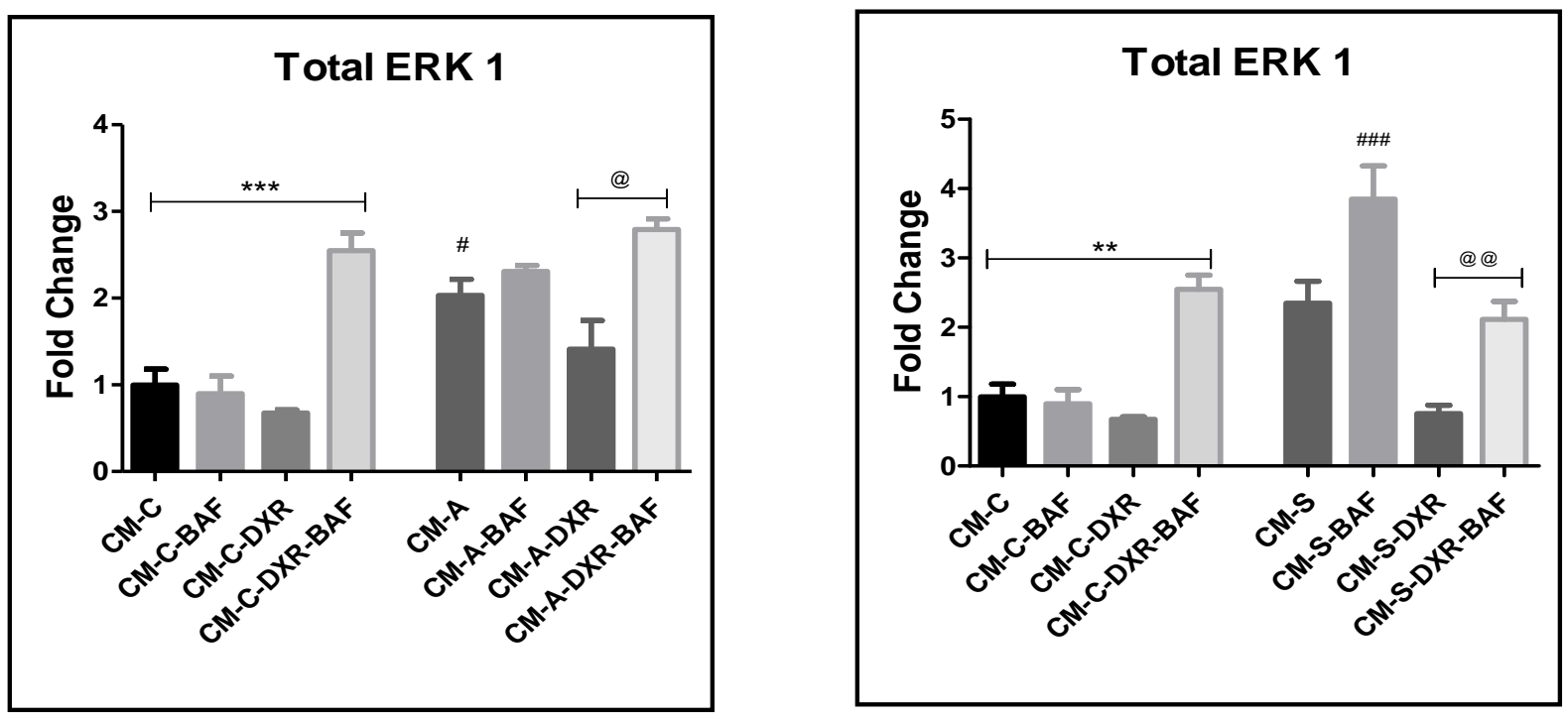

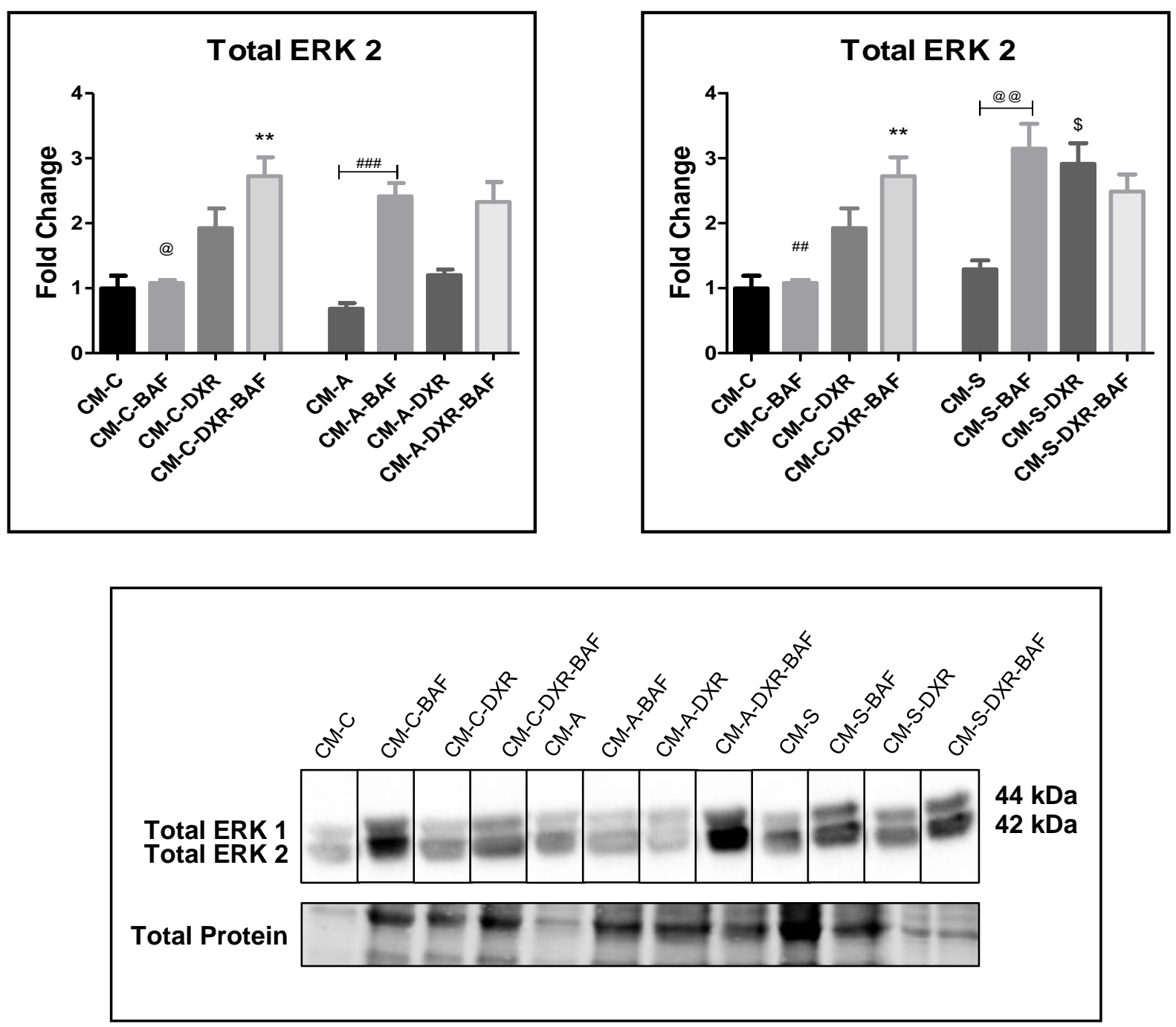

Figure 3.25.2: Western blot analysis to detect total ERK 1 and ERK 2 in E0771 cells. E0771 cells were seeded and treated with either control, apoptosis or senescence conditioned media collected from MEFs as described earlier or treated with $\mathrm{CM}$ and $1 \mu \mathrm{M}$ DXR for 24 hours, alongside with Bafilomycin to inhibit autophagy. Proteins $(50 \mu \mathrm{g})$ from E0771 cells were separated by SDS-PAGE and transferred to a PVDF membrane for western blot analysis. Results are presented as means \pm SEM $(n=3)$. ERK expression is shown for control CM (lane 1), control CM and Baf (lane 2), control CM and DXR (lane 3), control CM, DXR and Baf (lane 4), apoptosis CM (lane 5), apoptosis CM and Baf (lane 6), apoptosis and DXR (lane 7), apoptosis CM, DXR and Baf (lane 8), senescence CM (lane 9), senescence CM and Baf (lane 10), senescence CM and DXR (lane 11) and senescence CM, DXR and Baf (lane 12). ERK 1: For control and apoptosis $\mathbf{C M}$ the asterisk $\left({ }^{*}\right)$ denotes a significant increase in CM-C-DXR-Baf when compared to CM-C $(p<0.05)$, (\#) denotes a significant decrease in CM-C-DXR relative to CM-ADXR $(p<0.001)$ and $(@)$ denotes a significant increase in CM-A-DXR-Baf relative to CM-A-DXR $(p<0.001)$. For control and senescence $\mathbf{C M}\left(^{*}\right)$ denotes a significant decrease in CM-C-DXR-Baf $(p<0.01)$, the $(\#)$ denotes a significant increase in CM-S-Baf relative to CM-C-Baf $(p<0.001)$ and (@) denotes a significant increase in CM-S-DXR-Baf relative to CM-S-DXR $(p<0.001)$. ERK 2: For control and apoptosis CM the asterisk $\left({ }^{*}\right)$ denotes a significant increase in CM-C-DXR-Baf relative to CM-C 
$(p<0.01)$, (@) denotes a significant decrease in CM-C-Baf relative to CM-A-Baf $(p<0.05)$ and $(\#)$ denotes a significant increase in CM-A-Baf relative to CM-A $(p<0.001)$. For control and senescence CM $(\#)$ denotes a significant decrease in CM-C-Baf relative to CM-S-Baf $(p<0.001),\left({ }^{*}\right)$ denotes a significant increase in CM-S-DXR-Baf relative to the control ( $p<0.01)$; (@) denotes a significant increase in CM-S-Baf relative to CM-S $(p<0.01)$ and $(\$)$ denotes a significant increase in CM-S-DXR relative to CM-C-DXR $(p<0.05)$.

The ratio of phosphorylated ERK 1 and total ERK 1 was then determined. For apoptosis, the expression of $p E R K 1 /$ total ERK1 was significantly decreased $(p<0.01)$ in all apoptosis $C M$ groups, relative to the control (CM-C). The expression was also decreased in control $\mathrm{CM}$ treated with DXR and Bafilomycin when compared to control CM treated only with DXR $(p<0.01)$. Furthermore, the expression of $p E R K 1 /$ total ERK 1 was also significantly decreased in apoptosis CM treated with DXR when compared to the control CM treated with DXR $(p<0.05)$. For senescence, the expression was significantly decreased in all senescence $C M$ groups, except CM-S-DXR-Baf $(p<0.01)$. Additionally, senescence CM treated with DXR and Bafilomycin (CM-S-DXR-Baf) was significantly increased when compared to the control CM treated with DXR and Bafilomycin (CM-C-DXR-Baf) $(p<0.05)$.
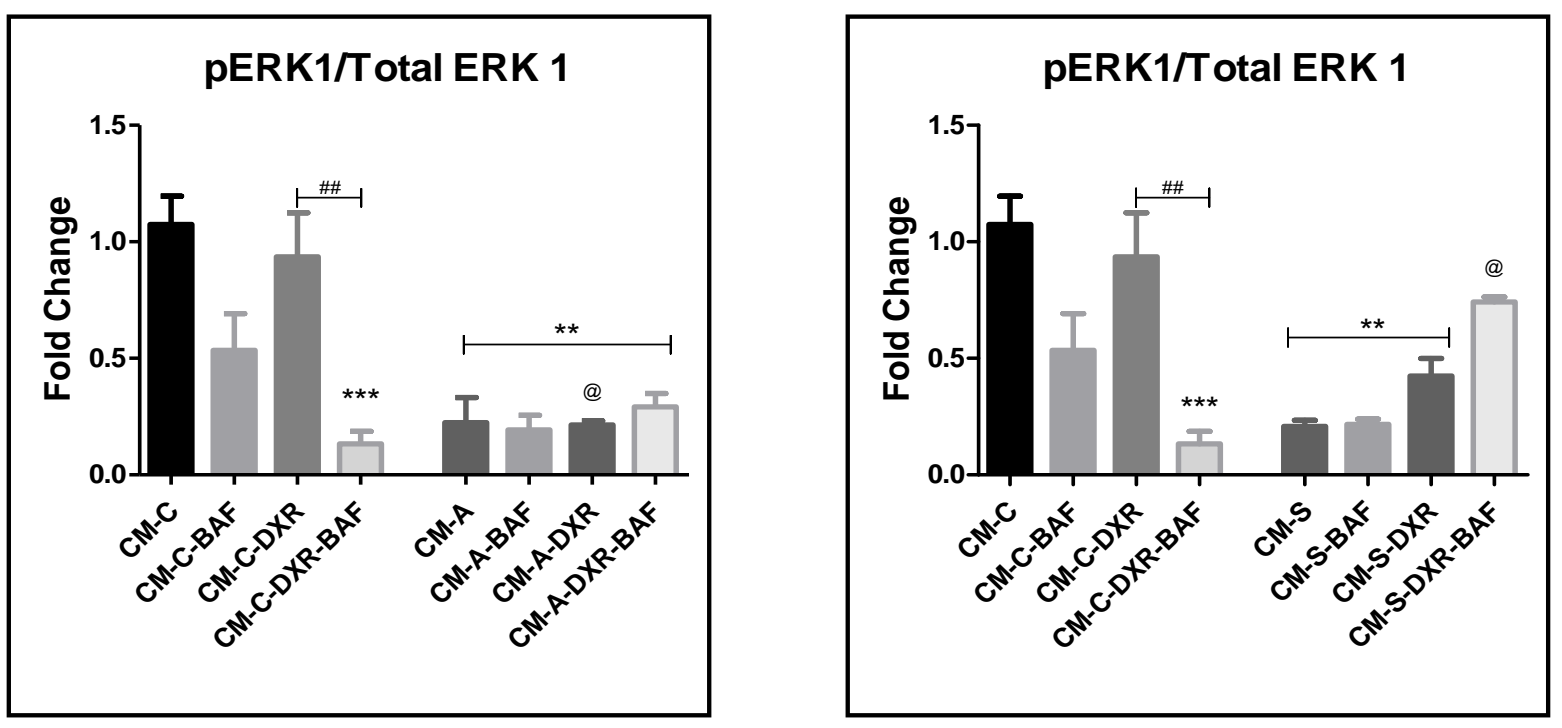

Figure 3.25.3: pERK 1/total ERK 1 in E0771 cells. The ratio of phosphorylated ERK 1 and total ERK 1 was determined. For control and apoptosis $\mathbf{C M}\left(^{*}\right)$ denotes a significant decrease in CM-C-DXRBaf $(p<0.001)$ and all apoptosis CM $(p<0.01)$; (\#) denotes a decrease in CM-C-DXR-Baf when compared to CM-C-DXR ( $<<0.01)$ and $(@)$ denotes a decrease in CM-A-DXR when compared to CM$C-D X R(p<0.05)$. For control and senescence $C M\left(^{*}\right)$ denotes a significant decrease in CM-C-DXRBaf $(p<0.001)$ and in CM-S, CM-S-Baf and CM-C-DXR relative to the control $(p<0.01)$; (\#) denotes a 
decrease in CM-C-DXR-Baf when compared to CM-C-DXR $(p<0.01)$ and $(@)$ denotes an increase in CM-S-DXR-Baf when compared to CM-C-DXR-Baf $(p<0.05)$.

The ratio for phosphorylated ERK 2 and total ERK 2 was also determined. For apoptosis, the expression of pERK 2/total ERK 2 was significantly increased in apoptosis CM treated with DXR $(C M-A-D X R)$ relative to the control $(p<0.01)$ and apoptosis CM $(p<0.01)$. Apoptosis CM treated with DXR and Bafilomycin (CM-A-DXR-Baf) was also significantly decreased when compared to senescence CM treated with DXR (CM-S-DXR) $(p<0.01)$. For senescence, the expression was significantly increased in senescence CM treated with DXR (CM-S-DXR) $(p<0.001)$ and senescence CM treated with DXR and Bafilomycin (CM-S-DXR-Baf) $(p<0.05)$ relative to the control as well as senescence CM (CM-S) $(p<0.001$ and $p<0.01)$.
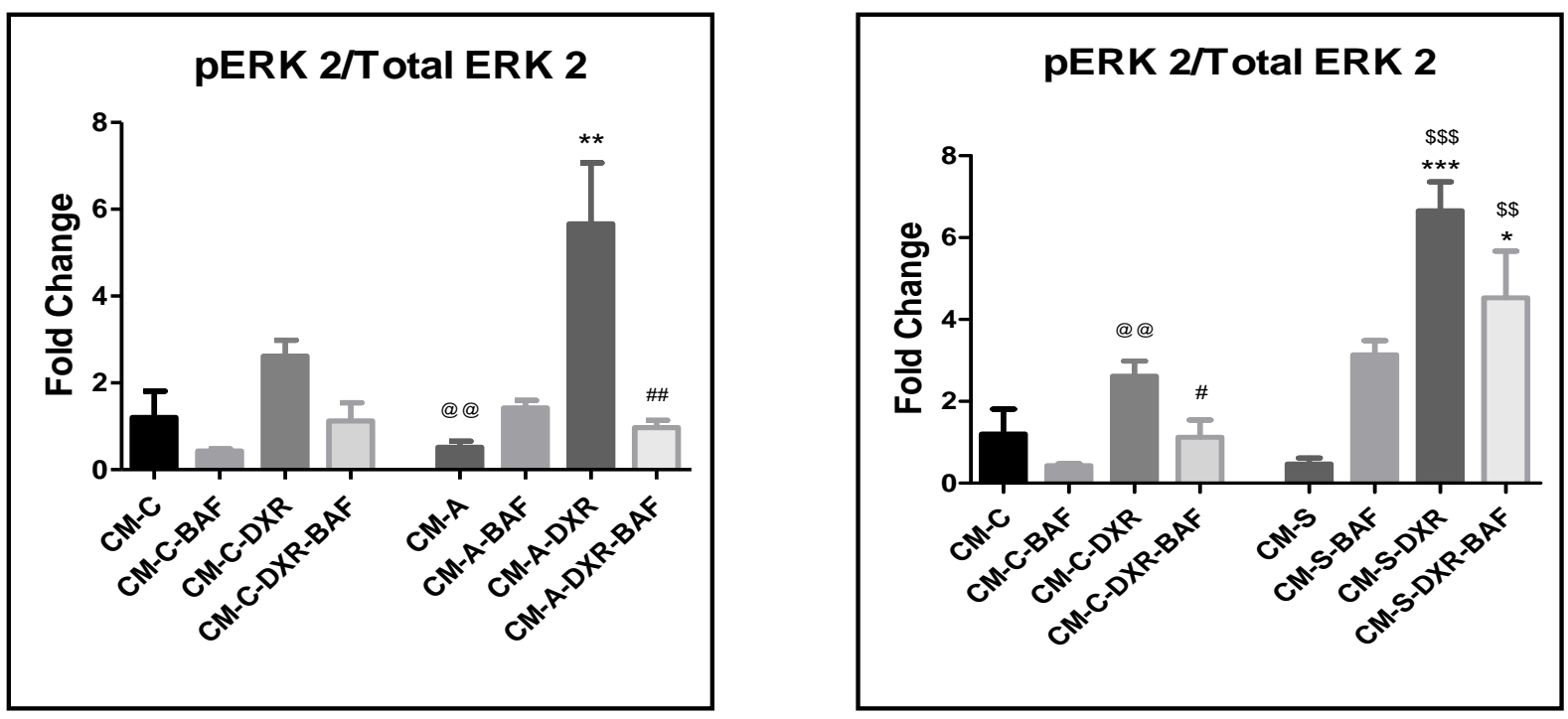

Figure 3.25.4: pERK 2/total ERK 2 in E0771 cells. The ratio of phosphorylated ERK 2 and total ERK 2 was determined. For control and apoptosis $\mathbf{C M}\left(^{*}\right)$ denotes a significant increase in CM-A-DXR relative to CM-C ( $<<0.01)$; (\#) denotes a decrease in CM-C-DXR-Baf when compared to CM-C-DXR $(p<0.01)$ and $(@)$ denotes a decrease in CM-A when compared to CM-C-DXR $(p<0.01)$. For control and senescence CM $\left({ }^{*}\right)$ denotes a significant increase in CM-S-DXR-Baf $(p<0.05)$ and CM-S-DXR $(p<0.001)$ relative to the control; $(\$)$ denotes an increase in CM-S-DXR $(p<0.001)$ and CM-S-DXR-Baf $(\mathrm{p}<0.01)$ when compared to CM-S; (\#) denotes a decrease in CM-C-DXR-Baf when compared to CMS-DXR-Baf $(p<0.05)$ and $(@)$ denotes a significant decrease in CM-C-DXR when compared to CM-S$\operatorname{DXR}(p<0.01)$. 


\subsection{Autophagy Immunocytochemistry: Quantification of LC3 and p62 puncta}

\subsubsection{LC3 II stain following conditioned media and DXR treatment with autophagy inhibition}

An Immunocytochemistry stain was used to confirm the results obtained from the LC3 western blot expression. For apoptosis, all groups were significantly increased when compared to the control $(p<0.001)$. The expression of LC3 was also increased in the control CM treated with DXR and Bafilomycin when compared to control CM treated with DXR $(p<0.05)$. For senescence, the control CM treated with DXR as well as DXR and Bafilomycin was significantly increased when compared to the control $(p<0.01$ and $p<0.001)$. Furthermore, LC3 was significantly decreased in senescence CM treated with DXR and Bafilomycin when compared to the control CM treated with DXR and Bafilomycin $(p<0.05)$.
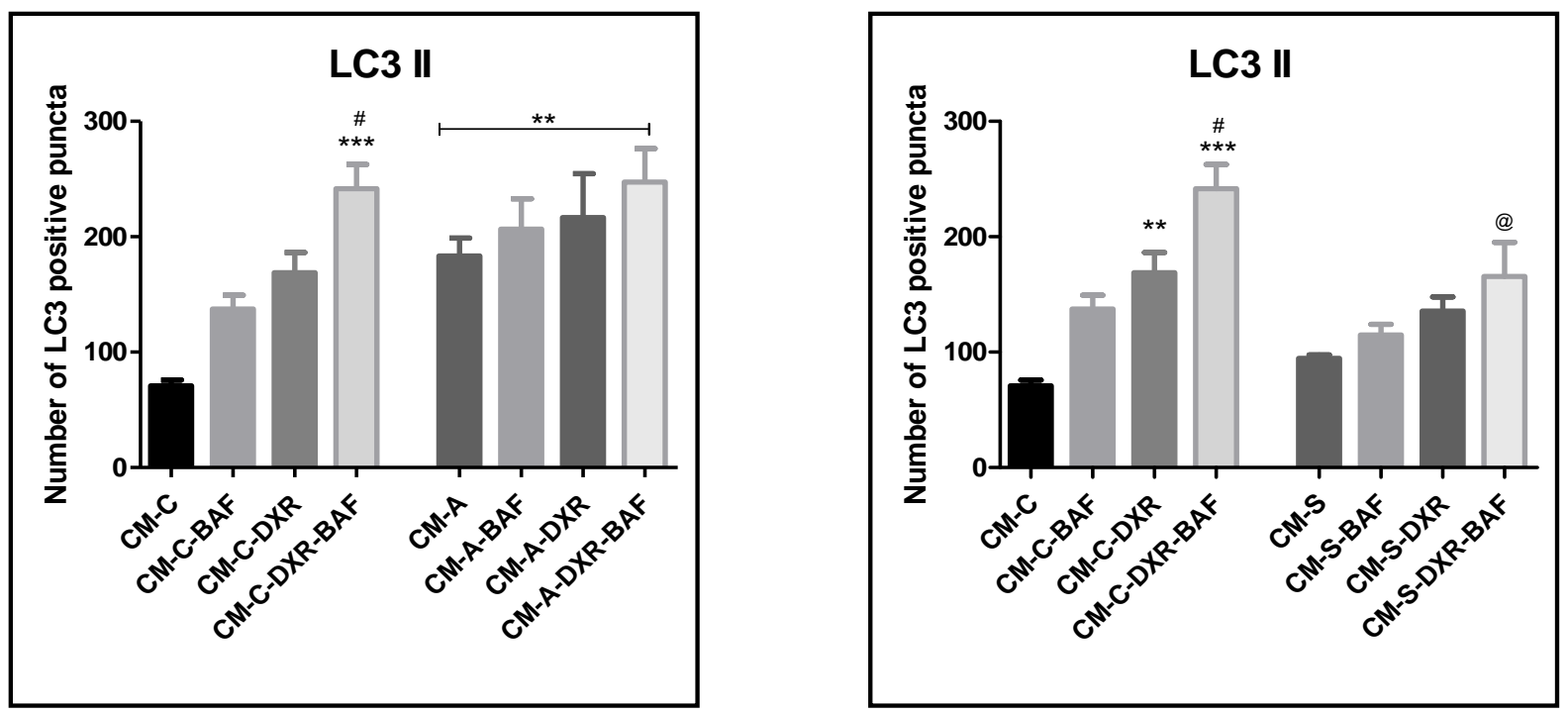

Figure 3.26.1: Immunocytochemistry analysis of LC3 expression in E0771 cells. E0771 cells were seeded onto coverslips and treated with either control, apoptosis or senescence conditioned media collected from MEFs as described earlier or treated with CM and $1 \mu \mathrm{M}$ DXR for 24 hours, alongside with Bafilomycin to inhibit autophagy. The cells were fixed and LC3 primary antibody was added, followed by overnight incubation. After incubation with secondary antibody, the cells were imaged. Results are presented as means \pm SEM $(n=3)$. For control and apoptosis $\mathbf{C M}$, the asterisk $\left(^{*}\right)$ denotes a significant increase relative to the control $(p<0.01$ and $p<0.001)$ and $(\#)$ denotes a significant increase relative to CM-C-DXR $(p<0.05)$. For control and senescence $\mathbf{C M},\left(^{*}\right)$ denotes a significant increase relative to the control $(p<0.01$ and $p<0.001)$; (\#) denotes a significant increase relative to CM-C-DXR $(p<0.05)$ and $(@)$ denotes a significant decrease relative to CM-C-DXR-Baf $(p<0.05)$. 
3.4.1.1 Representative images for LC3 puncta quantification
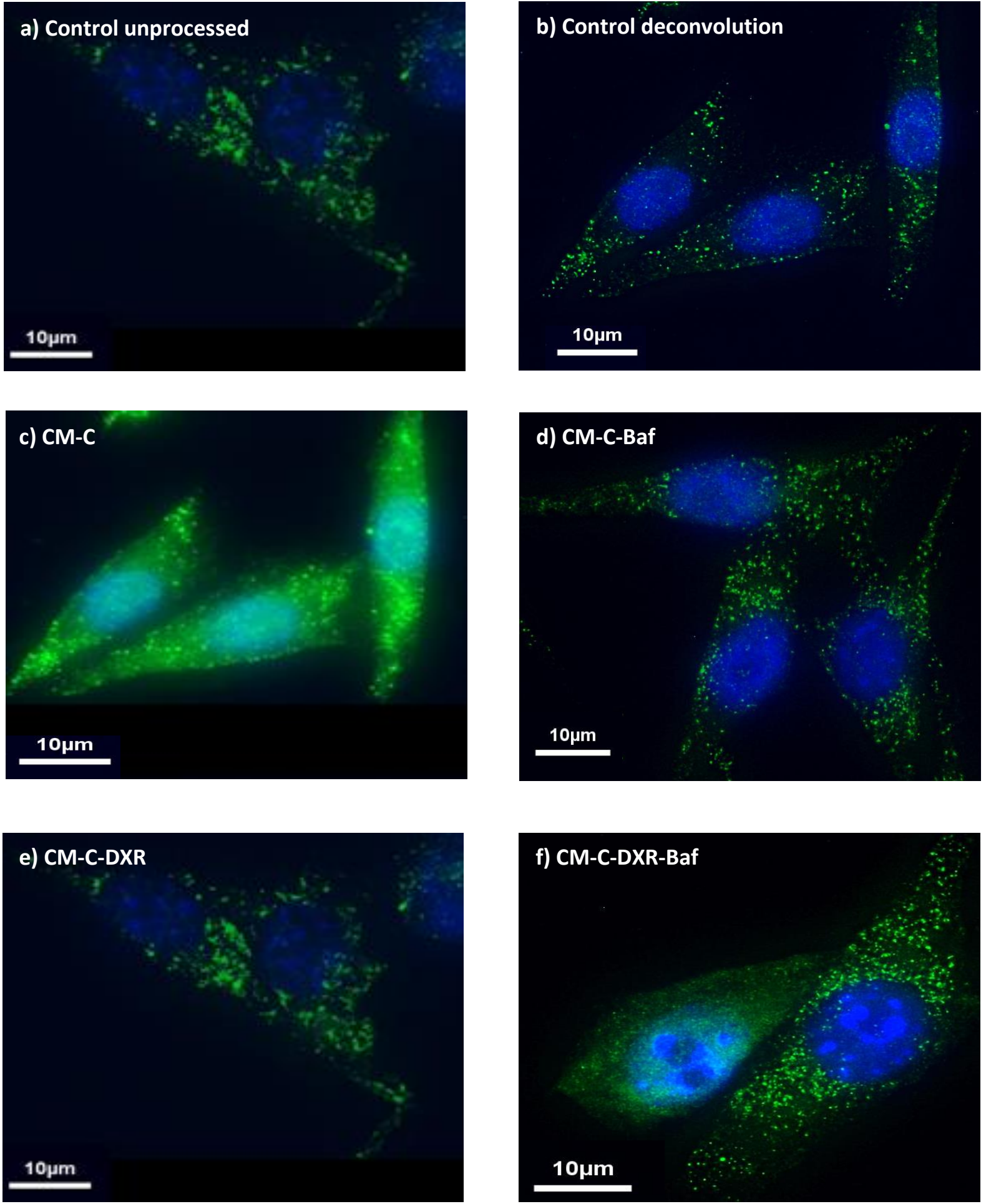

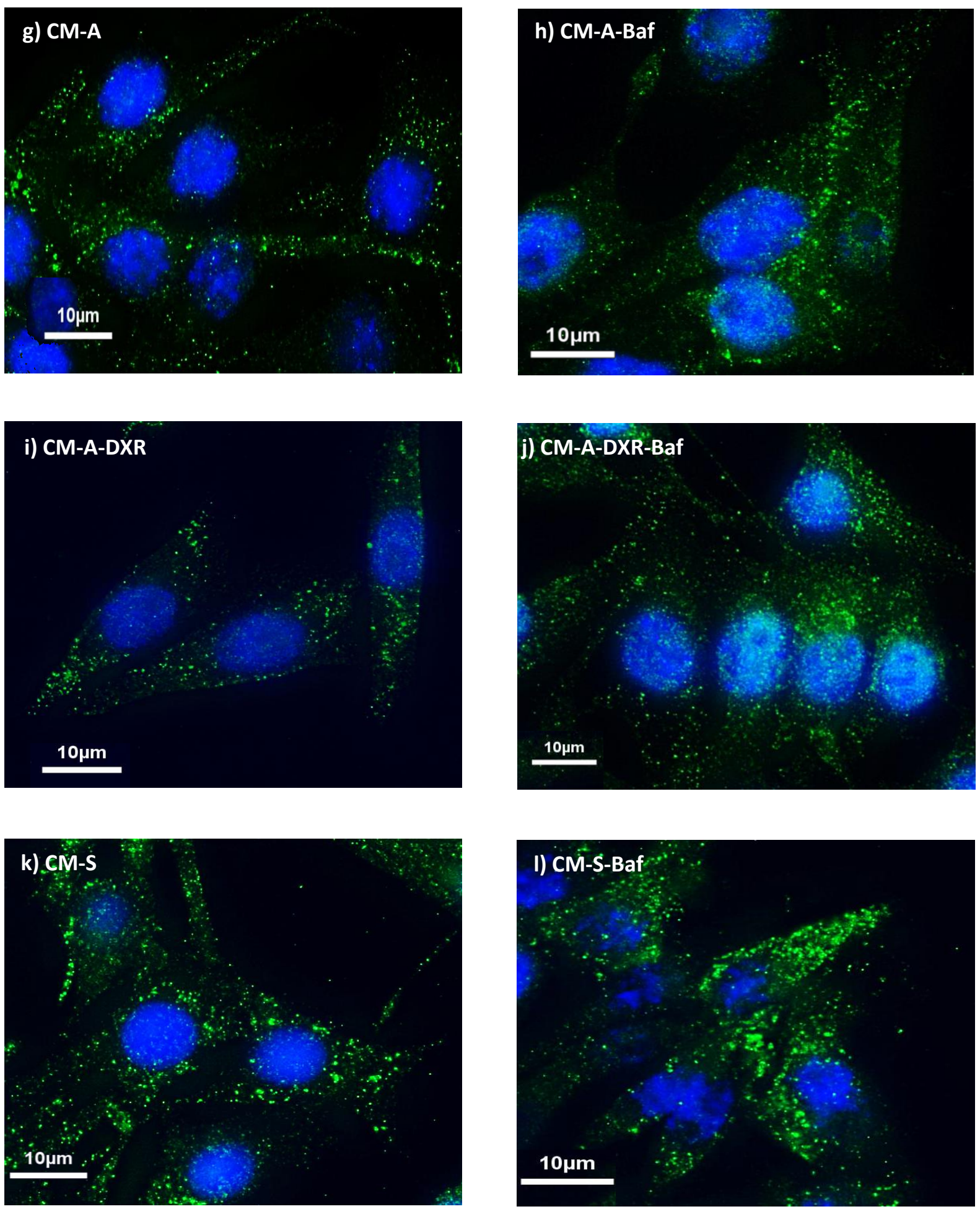

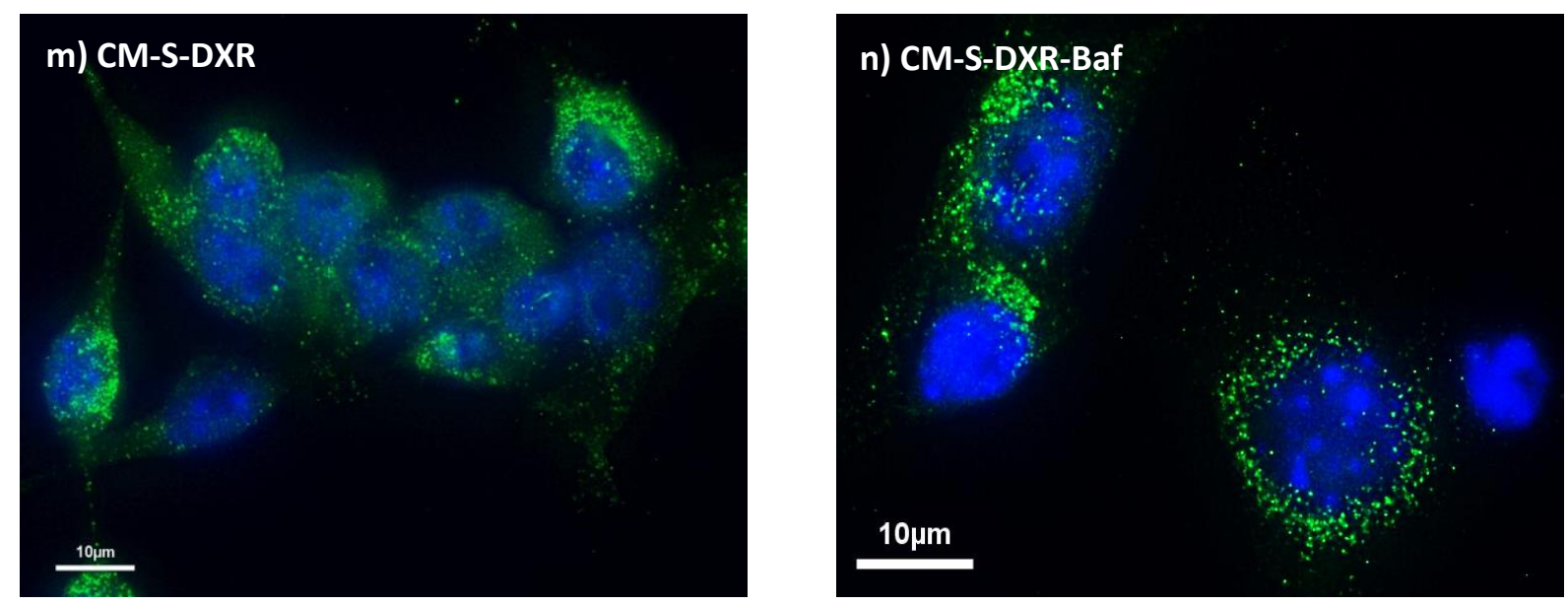

Figure 3.26.1.1: Representative images of LC3 puncta in E0771 cells following CM and DXR treatment and autophagy inhibition. E0771 cells were seeded and treated with control CM (as previously described) and DXR for 24 hours and Bafilomycin was added 4 hours prior to the staining protocol (c-f). The same protocol was followed for apoptosis CM (g-j) and senescence CM (k-n).

\subsection{2 p62 stain following conditioned media and Doxorubicin treatment with autophagy inhibition}

An Immunocytochemistry stain was used to confirm the results obtained from the p62 western blot expression. For apoptosis, all apoptosis CM and control CM treatment groups were significantly increased when compared to the control $(p<0.001)$. The expression of $p 62$ was also increased in all senescence CM treatment groups when compared to senescence CM $(p<0.001)$. For senescence, the control CM treated with DXR as well as DXR and Bafilomycin and senescence CM treated with DXR as well as DXR and Bafilomycin was significantly increased when compared to the control $(p<0.001$ and $p<0.01)$. Furthermore, $p 62$ was significantly increased in senescence CM treated with DXR as well as DXR and Bafilomycin when compared to senescence CM $(p<0.001)$. 

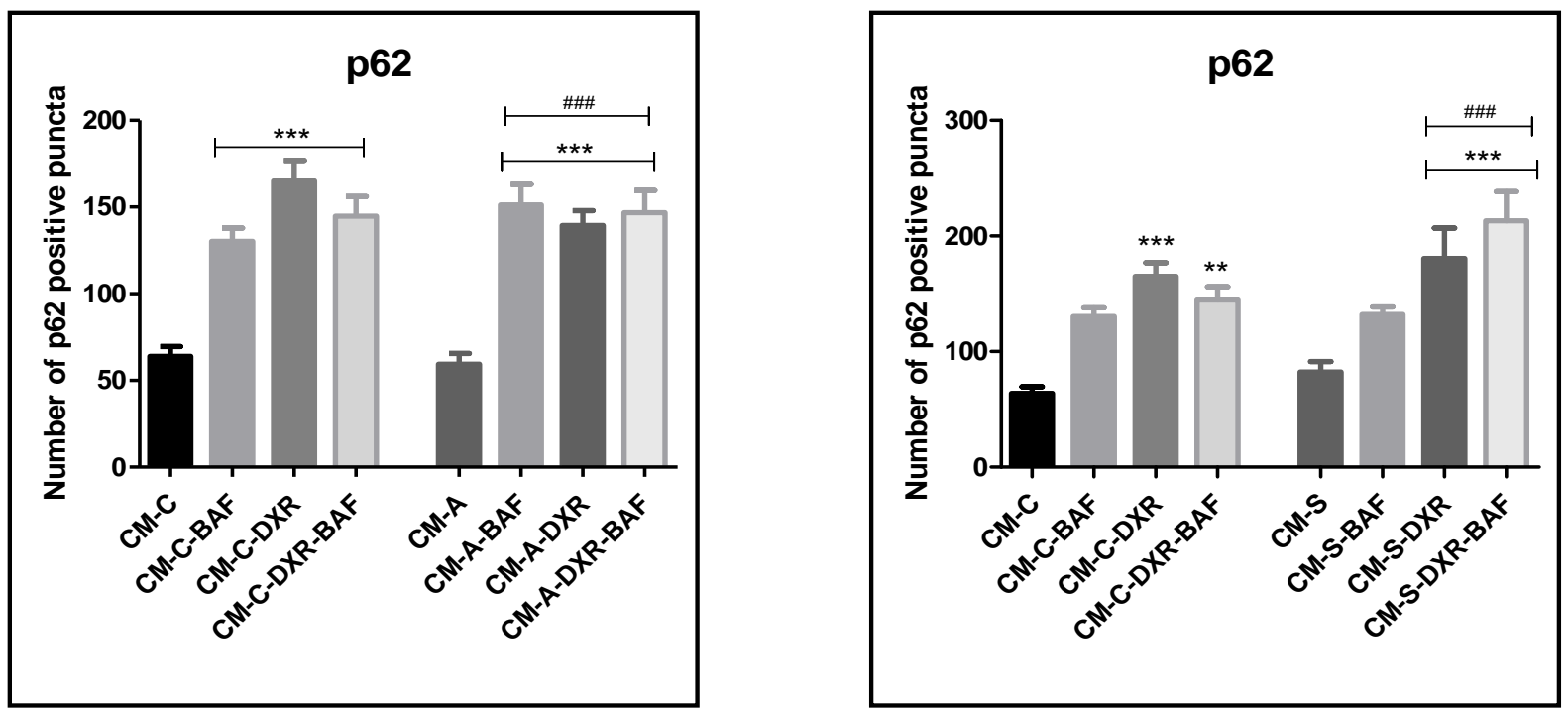

Figure 3.26.2: Immunocytochemistry analysis of p62 expression in E0771 cells. E0771 cells were seeded onto coverslips and treated with either control, apoptosis or senescence conditioned media collected from MEFs as described earlier or treated with CM and $1 \mu \mathrm{M}$ DXR for 24 hours, alongside with Bafilomycin to inhibit autophagy. The cells were fixed and p62 primary antibody was added, followed by overnight incubation. After incubation with secondary antibody, the cells were imaged. Results are presented as means \pm SEM $(n=3)$. For control and apoptosis $\mathbf{C M}$, the asterisk $\left({ }^{*}\right)$ denotes a significant increase relative to the control $(p<0.001)$ and $(\#)$ denotes a significant increase relative to CM-S $(p<0.001)$. For control and senescence $\mathbf{C M},\left(^{*}\right)$ denotes a significant increase relative to the control $(p<0.01$ and $p<0.001)$ and $(\#)$ denotes a significant increase relative to CM-S $(p<0.001)$.

\subsubsection{Representative images for p62 puncta quantification}
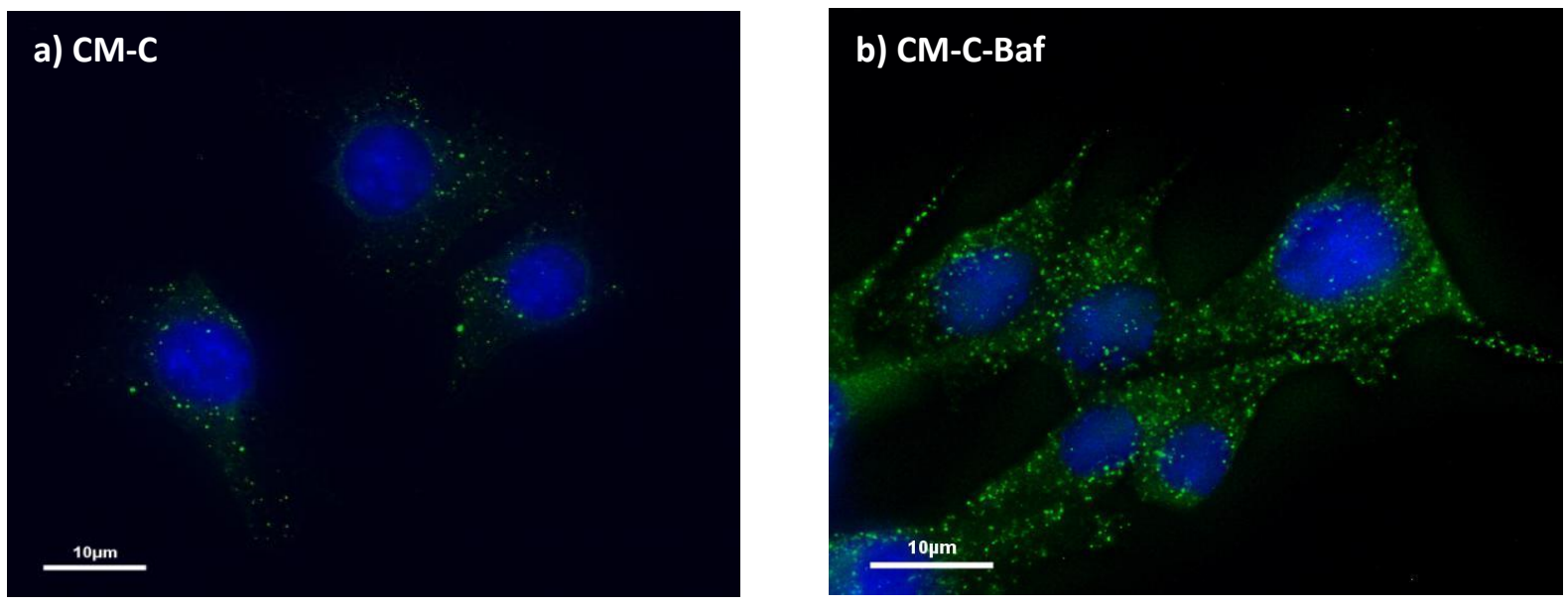

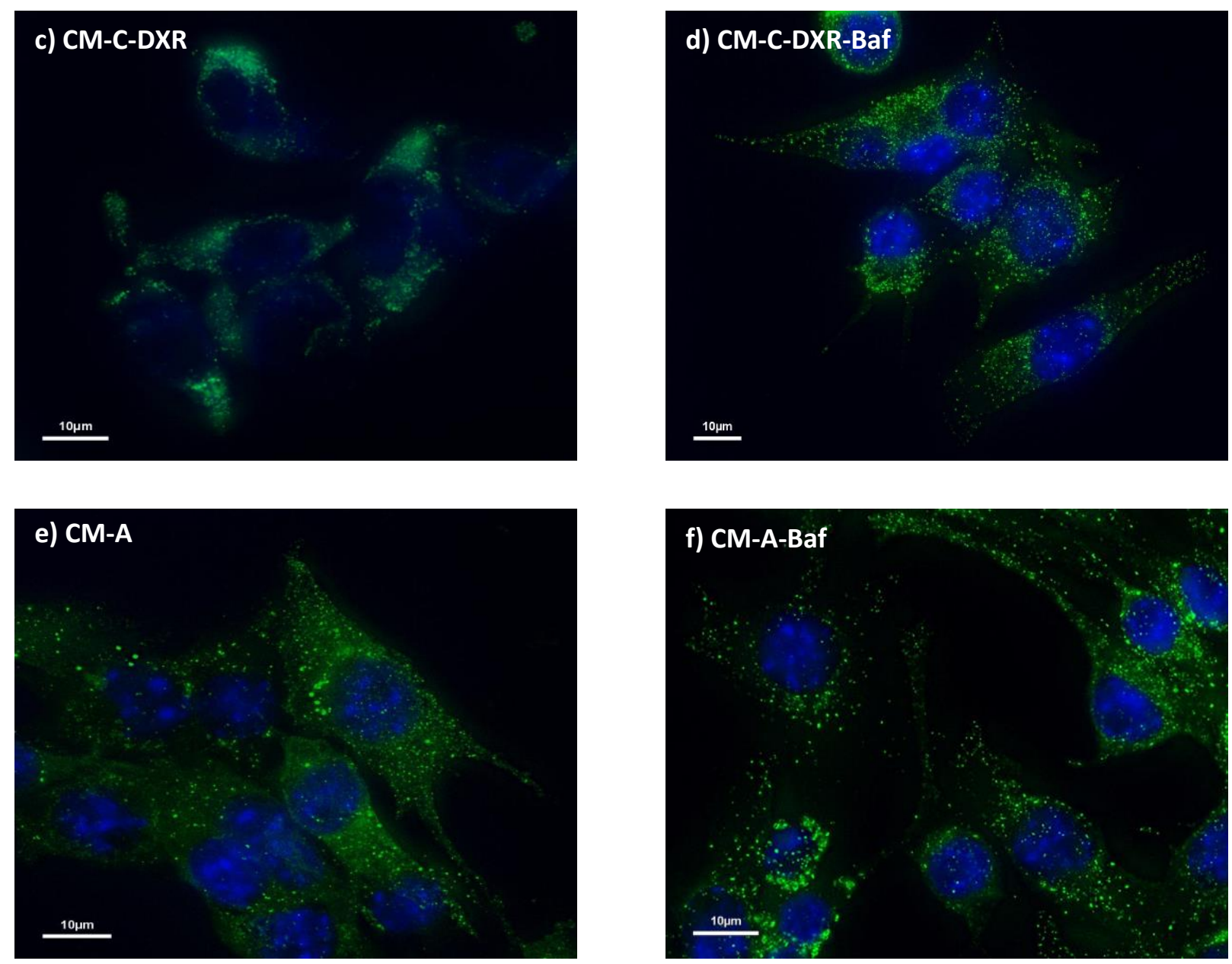

\section{g) CM-A-DXR}

h) CM-A-DXR-Baf 

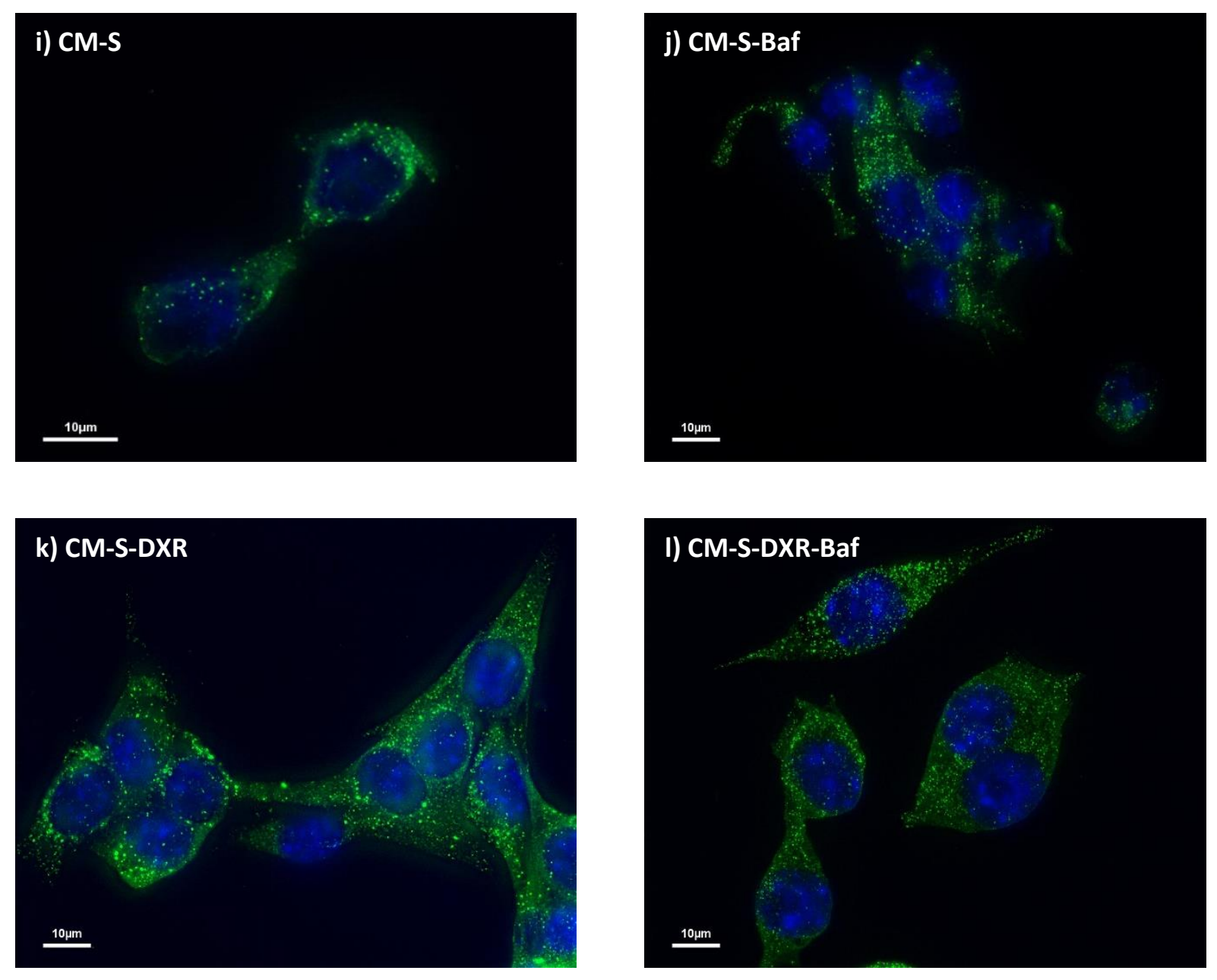

Figure 3.26.2.1: Representative images of p62 puncta in E0771 cells following CM and DXR treatment and autophagy inhibition. E0771 cells were seeded and treated with control CM (as previously described) and DXR for 24 hours and Bafilomycin was added 4 hours prior to the staining protocol (a-d). The same protocol was followed for apoptosis CM (e-h) and senescence CM (i-l). 


\section{Chapter 4}

\section{Discussion}

The most common cancer among women in both developed and developing countries is breast cancer and many treatment strategies are therefore being developed to reduce oncogenic transformation. (Desantis et al., 2016). A major treatment strategy currently used worldwide is chemotherapeutic drugs, such as DXR. These chemotherapeutic drugs function by damaging DNA, which has an influence on the cell's ability to divide. In response to the DNA damage, cancer cells can undergo apoptosis or senescence, depending on the dosage (Amornsupak et al., 2014). Cancer cells proliferate at faster rates than normal cells in the environment, which makes them more susceptible to apoptosis induced by these drugs (Vasillev et al., 2017). However, lower dosages or concentrations of DXR also target normal cells in the tumour microenvironment and these cells often induce a senescent state. These senescent cells actively communicate with neighbouring cells and the tumour microenvironment (TME), through the secretion of molecules that constitute the senescence associated secretory phenotype (SASP) such as cytokines, chemokines, and growth factors (Coppé et al., 2014). Senescent fibroblasts in the TME could therefore secrete certain factors that could promote the transformation and proliferation of cancer cells. The aim of this study was therefore to assess the effects of conditioned media harvested from senescent and apoptotic fibroblasts on breast cancer cells and to determine whether autophagy inhibition contributes to treatment resistance in cancer cells.

\subsection{Induction of apoptosis in MEFs}

\subsubsection{Cell viability}

An MTT assay was used to assess the cell viability of MEFs following a 24-hour treatment with DXR. In MEFs, $1 \mu \mathrm{M}$ to $10 \mu \mathrm{M}$ DXR significantly decreased cell viability after the 24-hour treatment (Figure 3.1).

No significant differences in cell viability were detected when MEFs were treated with dosages lower than $1 \mu \mathrm{M}$. This agrees with previous studies which showed that MEFs treated with 0.2 $\mu \mathrm{g} / \mathrm{ml}$ DXR for 17 to 18 hours failed to undergo apoptosis (Attardi et al., 2000). The data is also supported by the study of Park et al, where the authors treated MEFs with DXR ranging 
between 1 and $8 \mu \mathrm{M}$, for 24 hours. $1 \mu \mathrm{M}$ and $2 \mu \mathrm{M}$ DXR significantly decreased cell viability. Higher concentrations of DXR had no significant effects (Park et al., 2012).

\subsubsection{Markers of apoptosis}

Western blot analyses were used to indicate whether apoptosis occurred in MEFs 24 hours after DXR treatment. DXR is often associated with ROS production, which plays an important part in caspase activation to serve as primary mediators for the apoptosis pathway. Cleaved caspase 3 is used as a central marker to indicate if the caspase cascade has been activated and follows activation of either the extrinsic pathway (caspase 8) or the intrinsic mitochondrial pathway (caspase 9) (Wei et al., 2000).

Total Caspase 8 expression significantly decreased and cleaved caspase 8 significantly increased in the DXR treated MEFs when compared to the control. Similar results were obtained by Wei et al who treated MEFs with $3 \mu \mathrm{M}$ of DXR and found increased levels of caspase 8 cleavage in the DXR treated cells. The authors also concluded that higher concentrations of DXR (5 $\mu \mathrm{M}$ to $10 \mu \mathrm{M})$ can further increase the activation of caspase 8 in these cells.

No significant changes in expression of cleaved caspase 9 was observed after the 24-hour treatment period. Contradictory to our findings however, Kuida et al concluded that embryonic fibroblasts from caspase 9-null mice are resistant to apoptosis induced by DNA damaging agents. The authors therefore concluded that the activation and cleavage of caspase 9 is an important mechanism for apoptosis induction. Based on the current study, $1 \mu \mathrm{M}$ of DXR only activated the extrinsic pathway efficiently in MEFs, by activating caspase 8 to recruit procaspase 3 or 7 to induce apoptosis.

We have further demonstrated a decrease in total caspase 3 expression and a significant increase in cleaved caspase 3 following 24-hour treatment with $1 \mu \mathrm{M}$ DXR (Figure 3.4). These results are supported by the work of Weigh et al, who found that there is a time frame between 8 and 12 hours for DXR to induce caspase activation in MEFs, when MEFs are treated with 3 $\mu \mathrm{M}$ DXR.

The significant increase in caspase 3 activation and cleavage was however not supported by an increase in PARP activation and cleavage. Instead, PARP activation and cleavage significantly decreased after 24-hour DXR treatment (Figure 3.5). This finding was unexpected since Caspase 3 cleavage normally induces PARP activation and cleavage (Munoz-Gamez et al., 2005). These results also contradict the findings of Munoz-Gamez et al, who indicated 
that after caspase 3 activation, there was a significant increase in PARP cleavage (MunozGamez et al., 2005). The differences in the obtained results could possibly be based on treatment duration. The above-mentioned authors assessed PARP cleavage after a 72-hour treatment period. It could therefore be possible that the 24-hour treatment period is not substantial enough to induce PARP cleavage specifically in MEFs.

In response to genotoxic stress and DNA damage, p53 is rapidly increased in MEFs. This occurrence led Ding et al to suggest that when p53 is activated, it is able to recruit the assembly of a caspase 8 activating process by inducing protein-protein interactions in the cell. Caspase 8 would therefore be able to target caspase 9 through proteolysis, to activate caspase 3 and induce apoptosis (Ding et al., 2000).

In summary, these results support the idea that more than one mechanism is able to induce programmed cell death and that these mechanisms are highly dependent on the death stimulus as well as the cell type being investigated.

\subsection{Induction of senescence in MEFs}

\subsubsection{Senescence associated B-galactosidase activity}

A SA-B-gal assay was performed to measure endogenous lysosomal beta-galactosidase activity in senescent MEFs. MEFs were treated with $2 \mu \mathrm{M} D X R$ for 4 hours (Figure 3.6). After the treatment period, MEFs were incubated for 12 days before the stain was clearly visible. The number of senescent cells (whole cells that were stained bright blue) was then compared to the total number of cells in the cell population. A cell population is considered to be senescent when $80 \%$ of the population is positively stained. However, based on the results obtained from different sample areas of the cell population, we were unable to refer to the population as being senescent. The results do however show that DXR is capable of inducing senescence in a large portion of the cell population. The findings of Ghosh and co-workers support the ability of DXR to induce senescence in MEFs. The authors used a DXR treatment period of four days and found an increase in the number of SA-B-gal positive cells in fibroblasts and endothelial cells (Ghosh et al., 2016). The findings of Ghosh et al was however significant, but this could indicate that treatment duration is a main determinant in whether or not senescence will be induced in most of the cell population. However, Te Poele and colleagues obtained contradicting results. An increase in SA-B-gal staining was observed in $41 \%$ of breast tumours after DXR treatment, but the stain was confined to tumour cells and no detection in normal tissue was present (te Poele et al., 2002). These contradictory findings therefore 
highlight the importance of optimizing senescence induction for different cell lines. DXR therefore has the ability to induce senescence in many cells, including cancerous and noncancerous cell lines.

\subsubsection{Markers of senescence}

MCM2 is part of the mini chromosome maintenance protein complex and plays an important role in DNA replication and cell proliferation (Lei, 2005). Once senescence is induced in cells, the cells are unable to proliferate. The expression of MCM2 was therefore used to assess the proliferative capacity of MEFs after DXR treatment. Following treatment with $2 \mu M$ DXR for 4 hours, MEFs were incubated for different time periods (day 1, 2, 4, 8 and 12). There was a significant decrease in MCM2 expression on day 8 and 12 when compared to both the control and day 1 groups (Figure 3.7). Based on this finding, it can be concluded that the MEFs had lost their ability to proliferate after the treatment period. Chen et al subjected normal human fibroblasts to oxidative stress (can also be induced by DXR) by treating the cells with hydrogen peroxide for 2 hours. Similarly, to our findings, MCM2 expression significantly decreased in the near replicative senescent cells and was undetectable in the fully senescent cells (Chen et al., 2004).

The expression of p53 was increased on day 1 after the DXR treatment when compared to the control and then significantly decreased on day 8 and 12 when compared to both the control and day 1 groups. DNA damage induces the expression and activation of p53 and often results in the rapid accumulation thereof (Gire and Dulic, 2015). The tumour suppression functions of p53 are specifically linked with the activation of downstream targets to induce senescence or cell cycle arrest (Qian and Chen, 2013). Senescent cells are generally also characterized by the enhanced expression of p53 targets, like p21, a mediator of senescence. p53 therefore targets and activates p21 to induce senescence and this could possibly explain why there was an initial increase in p53 expression, followed by a significant decrease on day 8 and 12 (Figure 3.8). Furthermore, these results are also supported by a significant increase in p21 expression on day 12 (Figure 3.9), which possibly indicates that p53 tumour suppression functioning was reduced by $\mathrm{p} 21$. Several studies support these findings. Wang et al observed that when wild type MEFs are treated with $0.5 \mu \mathrm{M}$ and $1 \mu \mathrm{M} D X R, \mathrm{p} 21$ accumulation is significantly enhanced (Wang et al., 2012). Upon DNA damage, p53 levels in the cell are increased, which eventually causes an increase in p21 expression. p21 binds to and inhibits CDK, causing cell cycle arrest in the G1 phase. Attardi et al treated wild type MEFs with various DNA damaging agents, including DXR. Their results indicated that high levels of 
p53 expression was associated with low levels of p21. However, in p53 -/- MEFs, no changes in p21 expression was observed and cell cycle arrest could not be induced (Attari et al, 2004).

The overexpression of $\mathrm{p} 16$ has been reported in senescent fibroblasts in response to DNA damage and oxidative stress (Wu et al., 2007). DXR treatment gradually increased the expression of $\mathrm{p} 16$ from day 2 to day 12 . On day 12 , p16 expression was significantly increased when compared to the control (Figure 3.10). These findings are similar to those made by Spallarossa et al. The authors indicated that once endothelial progenitor cells (EPCs) are treated with $0.25 \mu \mathrm{M}$ DXR, p16 levels increased in SA-B-gal positive cells (Spallarossa et al., 2010). Additionally, Demaria and authors exposed MEFs to different concentrations of DXR to determine cell proliferation inhibition. Growth arrest was seen at $250 \mathrm{nM}$ DXR treatment. Senescence was also confirmed by the increase in p16 expression in cells as well as components of the SASP (IL-1 $\alpha$ and IL-6) (Demaria et al., 2017).

The Retinoblastoma ( $\mathrm{RB}$ ) protein is a vital tumour suppressor that blocks the progression of the cell cycle by inhibiting E2F transcription. RB expression was significantly increased on day 12 (Figure 3.11). Jackson et al indicated that after DNA damage occurs (induced by $1 \mu \mathrm{M}$ DXR), p130 levels (an RB family member) are elevated in MEFs after 24 hours of treatment (Jackson et al., 2006). He and co-workers exposed mouse embryonic stem cells to low doses of DXR (50 nM) for 48 hours. Similarly, RB expression was significantly increased after the treatment period (He et al., 2016). Further findings indicated that in response to DXR, RB expression was not significantly increased in p53 knock down cells. This data could possibly indicate that p53 mediated regulation of the RB protein occurs at the post-transcriptional level.

The markers of senescence influence each other during the senescence pathway. The expression of MCM2 decreased after treatment, which indicates that the MEFs started to lose their ability to proliferate, which supports the idea that senescent cells do not continue to grow. The p53, p21, p16 and RB proteins are tumour suppressor proteins that have the ability to block cell growth and induce senescence, and it was thus expected that the expression of these markers would increase. The SA-B-gal assay revealed that not all the MEFs in the cell population were senescent, which could have contributed to the results obtained for the senescence markers. If a larger proportion of the population were senescent, significant changes in the expression of p53 might have been observed, and possibly also at earlier time points for $\mathrm{p} 16$ and $\mathrm{p} 21$. 


\subsection{The paracrine effects of senescent and apoptotic MEFs on E0771 cells}

\subsubsection{Cell viability}

An MTT assay was used to assess the cell viability of E0771 cells after a 24-hour treatment period with apoptosis and senescence CM collected from MEFs with or without DXR. Cell viability was significantly reduced in CM groups treated with DXR $(p<0.05)$, however, senescence CM treated with DXR had a significant increase in cell viability when compared to the control and apoptosis CM treated with DXR (Figure 3.12).

Similarly, Amornsupak et al found that when MDA-MB-231 breast cancer cells were pretreated with conditioned media from breast cancer associated fibroblasts, the cancer cell cultures showed less cell death after a DXR $(5 \mu \mathrm{M})$ treatment period for 48 hours, when compared to cancer cells pre-treated with conditioned media from non-tumour associated fibroblasts (Amornsupak et al., 2014). These findings therefore support the ability of cancer cells to evade apoptotic cell death after DXR has been administered.

To the best of our knowledge, apoptosis and senescence CM treatment in breast cancer is a novel study, but the expression of apoptotic and senescence markers can be compared with other studies, which have similar results.

\subsubsection{Markers of apoptosis following conditioned media treatment}

Cytotoxic agents, like DXR, induces apoptosis by activating death signalling pathways in target cells (Fisher, 1994). Western blot analyses were therefore used to determine whether E0771 cells undergo apoptosis after the cells were treated with apoptosis and senescence CM, with or without DXR. As mentioned previously, DXR activates caspase activity, which in turn activates the execution of the apoptotic pathway.

In 2016, Pilco-Ferreto and Calaf found that caspase-8 (the death receptor signalling kinase) protein expression is significantly upregulated in MDA-MB-231 cells after DXR treatment, however, based on our findings, no significant changes were found in the expression of caspase 8 after the DXR treatment period in all CM groups ( $p>0.05$ ). Significant changes were observed in the decreased expression of caspase 8 in senescence CM treated with DXR relative to the control CM treated with DXR $(p<0.05)$, which could indicate that less apoptosis occurred in senescence CM (Figure 3.13). 
After the treatment period, no significant changes were observed in the expression of caspase 9 ( $p>0.05)$ (Figure 3.14). Pilco-Ferreto and Calaf showed that caspase 9 was significantly increased in MDA-MB-231 cells after a 48-hour treatment period with various concentrations of DXR. Caspase 9 is an initiator caspase involved in the intrinsic apoptotic pathway and the results obtained during our study suggests that Caspase 9 has not been activated due to the lack of caspase 9 cleavage after the treatment period, which could therefore suggest that only the extrinsic apoptosis pathway was activated during the 24-hour treatment period.

Caspase 3 is required for typical hallmarks of apoptosis like chromatin condensation and DNA fragmentation. In this study, caspase 3 expression was significantly decreased in senescence $C M$ treated with DXR $(p<0.05)$ however, no significant changes were observed in caspase 3 expression after treatment with DXR (Figure 3.15). Contradicting to these results, it was found in other studies that caspase 3 expression is significantly decreased in MCF-7 cells after a DXR treatment period for 48 to 72 hours (Yang et al., 2001; Pilco-Ferreto \& Calaf, 2016).

PARP is a conserved nuclear protein that binds to DNA strand breaks. Organisms with a PARP deficiency display impaired base extension repair as well as genomic instability, which indicates that PARP functions to respond to severe DNA damage (Shall \& de Murcia, 2001). DNA damage therefore increases PARP activity to allow cancer cells to repair DNA that has been damaged, to be able to continue growing. DXR is a powerful DNA-damaging agent, however, its direct effects on PARP activation has not fully been elucidated. PARP expression was significantly decreased in senescence $\mathrm{CM}$ as well as in senescence $\mathrm{CM}$ treated with DXR $(p<0.05)$ (Figure 3.16). Munoz-Gamez et al indicated that PARP is rapidly activated in MDAMB-231 cells after 60 minutes of DXR treatment (Munoz-Gamez et al., 2005). Additionally, Wang et al showed that Adriamycin (chemotherapeutic agent) induced PARP activation in MCF-7 cells, but did not have any effects on cleaved PARP after 24 hours (Wang et al., 2014).

The expression of most apoptosis markers was significantly decreased in senescence CM treated with DXR which is supported by the increase in cell viability in senescence CM treated with DXR, compared with both apoptosis and control CM. These findings therefore indicate that E0771 cells treated with senescence CM and DXR were able to evade apoptotic cell death and continue to grow, which supports the ability of senescent MEFs to secrete factors that promote treatment resistance.

\subsubsection{Markers of senescence following conditioned media treatment}

Western blot analyses were used to determine whether senescence was induced in E0771 cells after the treatment period. MCM2 plays an important part in the cell cycle during DNA 
replication and was therefore used as a proliferative marker to determine why the cancer cells have the ability to continue growing after DXR had been administered. The expression of MCM2 was significantly increased in senescence $\mathrm{CM}$ as well as senescence $\mathrm{CM}$ treated with DXR ( $p<0.05)$. (Figure 3.17). Similarly, Abe et al observed that once MCM2 translocation to the nucleus is inhibited, DXR-induced apoptosis is enhanced (Abe et al., 2015). Furthermore, Etemad-Moghadam et al stated that an increase in MCM2 gene expression is associated with a poor prognosis and an unfavourable response to DXR therapy (Etemad-Moghadam et al., 2013). The increased expression of MCM2 in the senescence CM treatment group therefore indicates that the cancer cells were able to proliferate, which is also supported by the decreased expression of several apoptosis markers. However, markers of senescence (a nonproliferative state) were also increased in the E0771 cells. The p16 protein was also assessed after the treatment period. p16 is a tumour suppressor protein which inhibits cyclin-D dependent protein kinases, by regulating the $\mathrm{G} 1$ phase to the S-phase during the cell cycle (Romagosa et al., 2011). The expression of p16 (Figure 3.19) and total RB (Figure 3.20) was increased in senescence CM with DXR $(p<0.05)$, which indicates that these cells were in a senescent state and were not able to grow, however, this finding is contradicted by the increased expression of MCM2. The increased expression of RB (a tumour suppressor protein that prevents excessive cell growth) is supported by the study of Jackson and Pereira-Smith who observed that RB and its family members were enhanced in MCF-7 cells after a DXR treatment period and the authors also concluded that the expression of any RB family members is sufficient to induce cell cycle arrest (Jackson \& Pereira-Smith, 2006). In 2011, Yang et al indicated that the upregulation of $\mathrm{p} 16$ promotes senescence in breast cancer cells (Yang et al., 2011).

The p53 protein is a tumour suppressive protein that plays a role in both senescence and apoptosis, as mentioned previously. The expression of p53 was significantly increased in apoptosis CM treated with DXR ( $p<0.05)$ (Figure 3.18). Oncul and Recan observed that p53 is activated in MCF-7 cells after low doses of DXR (800 nM) which led to cell cycle arrest in the G1 phase (Oncul \& Recal, 2017). When comparing cell viability in CM-A-DXR and CM-CDXR (Figure 3.12), no changes were observed. The increase in p53 expression could therefore indicate that cell growth was arrested in CM-A-DXR and the cells were not able to grow.

The expression of senescence markers therefore indicate that the breast cancer cells were able to proliferate even after DXR had been administered (MCM2), however, the expression of p16 and RB which maintain a senescent state in cells, was also increased, indicating that these cells were in a non-proliferative state. The contradicting data could be because the 
amount of senescence induction in MEFs was only $56 \%$ where literature has suggested that it should be $80 \%$. Furthermore, the treatment duration could also contribute to these findings.

\subsubsection{Markers of autophagy following conditioned media treatment}

Autophagy is a normal physiological process that maintains cellular homeostasis by digesting damaged cellular components in lysosomes (Rodriguez-Enriquez et al., 2004). Basal levels of autophagy are found in all cell types, but can also be induced by external stressors like chemotherapeutic drugs (Kondo \& Kondo, 2006; Maiuri et al., 2007). Studies suggest that autophagy promotes resistance to cell death within cancer cells, however, the role of autophagy in cancer remains controversial because of its cytotoxic or cytoprotective effect (Huang et al., 2016). Cell viability was significantly decreased in the control conditioned media groups once Bafilomycin (a late stage autophagy inhibitor) was added $(p<0.05)$ but was increased in apoptosis and senescence conditioned media $(p<0.01$ and $p<0.05)$ (Figure 3.21). This could therefore suggest that autophagy had a cytoprotective role towards cancer cells in the control conditioned media and a cytotoxic role towards cancer cells in the apoptosis and senescence media, indicating that the autophagic process was contributing to cancer cell death and not promoting treatment resistance.

During autophagy, LC3 in its cytosolic form (LC3 I) is conjugated to phosphatidylethanolamine to form LC3-phosphatidylethanolamine conjugate (LC3 II) and is therefore used to represent autophagic flux (Tanida et al., 2008). The western blot analyses of LC3 II indicated that it was significantly increased in conditioned media groups treated with Bafilomycin $(p<0.05)$ which represents autophagosome accumulation, as expected with autophagy inhibition (Figure 3.23). However, no significant changes in the expression of autophagic flux (defined as a measure of autophagic degradation activity) was observed (Loos et al., 2014). The immunocytochemistry results also show similar results, where LC3 II was increased in the CM groups treated with Bafilomycin but no changes in autophagic flux was observed $(p>0.05)$ (Figure 3.26.1). Similar to these results, Guo et al found that LC3 levels were significantly increased in MCF-7 cells after a treatment period with Chloroquine and high dosages of DXR (Guo et al., 2016).

p62 (or sequestosome 1) is an autophagy substrate that reports autophagy activity and has various roles in tumourigenesis and caspase activation (Liu et al., 2016). Studies also suggest that p62 is a selective cargo receptor that links ubiquitinated protein aggregates to autophagic machinery, through LC3 (Pankiv et al., 2007). Furthermore, although not significant, the expression of $\mathrm{p} 62$ was increased in CM-A-DXR-Baf when compared to CM-A-DXR indicating 
that a slight increase in autophagic flux had occurred ( $p>0.05)$. However, Loos et al indicated that the expression of p62 in western blot analysis should be plotted over time to represent autophagic flux (Loos et al., 2014). No significant changes of p62 was observed in the senescence conditioned media groups $(p<0.05)$. Similarly, Guo et al also indicated that $p 62$ is significantly increased in MCF-7 cells treated with Bafilomycin and DXR when compared to control groups (Guo et al., 2016). The authors therefore suggested that breast cancer cells in the presence of DXR upregulate p62 expression for survival. No significant changes in the immunocytochemistry analysis of p62 were observed to represent changes in autophagic flux ( $p>0.05)$ (Figure 3.26.2). However, the results obtained for $p 62$ from western blots and Immunocytochemistry differ and further assessment must be implemented. Several studies have demonstrated that weakly basic chemotherapeutic drugs, like DNA binding anthracyclines (DXR), can accumulate in lysosomes and is ultimately targeted to be degraded, which diminishes the ability of DXR to target the DNA of cancer cells. The sequestration of chemotherapeutics into lysosomes is therefore considered to be a mechanism of resistance in cancer cells (Hindenburg et al., 1989; Coley et al., 1993). As a result, the above mentioned could potentially explain why autophagy had a cytoprotective role towards cancer cells in the control conditioned media groups (Figure 3.21).

\subsubsection{Markers of proliferation following conditioned media treatment}

The expression of Akt and ERK was used to determine whether the E0771 cells were able to continue proliferating after the treatment period with conditioned media and DXR because the senescence conditioned media showed an increase in cell viability, when compared to the control and apoptosis groups (Figure 3.12). Lately, the role of Akt has received much interest in cancer research because it has the ability to suppress apoptosis. In 1998, Cardone et al indicated that Akt blocks and inhibits the functioning of caspase 9, which could provide the rationale why no caspase 9 cleavage was observed during our study (Figure 3.14) (Cardone et al., 1998). Similarly, to our results, Li et al observed that phosphorylated Akt was increased in MCF-7 cells one hour after exposing the cells to $1 \mu \mathrm{M}$ of DXR, followed by a robust increase in phosphorylated Akt after 24 hours, with no significant changes in total Akt (Li et al., 2005) (Figure 3.24.2). PI3K activation of Akt promotes the translocation of inactive Akt from the cytosol to the plasma membrane, through subsequent Akt activation through phosphorylation at the Thr308 and Ser473 sites. Akt plays an important role in many intracellular signalling systems like cell growth, proliferation and protection from apoptosis (Downward, 2004). Aktmediated cellular functions are regulated by many hormones and growth factors, including EGF, IGF and VEGF. Overall, growth factors that promote Akt activation regulates the 
transition of the cell cycle from the growth phase to the synthesis phase. Upstream effectors that activate Akt include PI3K and PTEN whereas downstream effectors mainly include mTOR. Carcinogenesis is highly dependent on external environmental stressors as well as genetic and epigenetic mutations to activate cellular oncogenes and at the same time inhibit tumour suppressor proteins. One of the hallmarks associated with an altered cellular phenotype is the overexpression/activation of Akt. Akt intracellular signalling maintains cellular biology which creates a steady equilibrium between tumour suppressor proteins and protooncogenes, however, if the balance is disturbed, proto-oncogenes prevail and cause the enhanced activation of Akt-related factors to contribute to a malignant phenotype (Karar \& Maity, 2011). As mentioned previously, the PI3K/Akt pathway is maintained at baseline activity. This is associated with an increased expression of p21 in the cellular nucleus which induces cell cycle arrest. During this period, malignant transformed cells take advantage of the temporary growth arrest to repair genomic DNA. In order to overcome this arrest, the Akt pathway is subsequently activated resulting in the nuclear rejection of p21 within the nucleus, to resume the proliferation program (Galluzi et al., 2012).

During our study, the ratio of phosphorylated Ser473 Akt to total Akt was used as a proliferative marker to determine Akt activation. Phosphorylated Akt was significantly increased in CM-A, CM-A-DXR and CM-S ( $<<0.05)$ (Figure 3.24.1), which is supported by the significant decrease in total Akt expression in the above-mentioned groups (Figure 3.24.2). A significant increase in the ratio between pAkt/Total Akt was observed in CM-A, CM-A-DXR and CM-S $(p<0.05)$ (Figure 3.24.3) which can be attributed to the significant increase in phosphorylated Akt, while total Akt levels remain unchanged. The increase in cell viability in CM-S-DXR (Figure 3.12) could therefore be attributed to the significant increase in Akt expression, which will allow the cancer cells to evade apoptotic cell death. Furthermore, total Akt expression was significantly increased in CM-C-DXR-Baf, CM-A-DXR-Baf and CM-S-DXR-Baf (Figure 3.24.1) with decreased expression for phosphorylated Akt (Figure 3.24.2) which leads us to believe that Akt activation had not been induced in these groups.

ERK1/2 (also known as MAPK3 or MAPK1) is a serine/threonine kinase found in most mammalian tissue, where ERK2 levels are generally higher than ERK1 (Boulton et al., 1990). Dual threonine and tyrosine residue phosphorylation activates both ERK1 and ERK2, at Thr202/Tyr204 for human ERK1 and Thr185/Tyr187 for human ERK2. Significant ERK activation requires phosphorylation at both sites (Ferrel \& Bahtt, 1997). pERK2 was significantly increased in the CM-A-DXR group $(p<0.001)$ indicating that significant ERK activation had occurred, however, p53 was also increased in this group (Figure 3.18) which could explain why apoptosis CM did not have a significant effect in cell viability changes (Figure 3.12). 
ERK $1 / 2$ is a therefore a key signalling molecule which has been extensively investigated as a drug target for cancer therapy because the Ras/Raf/MEK/ERK pathway promotes proliferation and malignant transformation of cancer cells (Sebolt-Leobolt, J, 2000). In 2012, Taherian et al observed that the expression of phosphorylated ERK1/2 and total ERK1/2 was decreased in MDA-MB-231 cells after a DXR treatment period, but was increased in MCF-7 cells and is therefore highly cell line specific (Taherian et al., 2012). The increased expression of ERK in MCF-7 cells therefore opposes the apoptosis pathway, which is similar to our findings where a significant increase in ERK expression was observed in the senescence conditioned media group treated with DXR, indicating that these cells were able to evade apoptotic cell death and is also supported by the lack of caspase 9 activation in both cell lines (Figure 3.25.4).

Not many studies have focused on the relationship between autophagy and ERK, but in 2013 Martinez-Lopez observed that the cellular availability of autophagic structures determines the degree of ERK phosphorylation. The authors speculated that LC3-II-positive membranes and ATG5-ATG12-positive pre-autophagosomes could serve as cellular signalling platforms that would allow spatial coordination of the Raf-MEK-ERK cascade to facilitate ERK phosphorylation. Furthermore, the authors indicated that an increase in cell death in autophagy deficient cells may have occurred from decreased ERK activity (Martinez-Lopez et al., 2013). This phenomenon was also observed during our study where cell death was increased in control CM groups (Figure 3.21) supported by the decreased expression of ERK in control CM treated with Bafilomycin (Figure 3.25.4) as well as the increased expression of LC3 II in Bafilomycin treated cells (Figure 3.26.1). Therefore, manipulating ATG5-ATG12 and LC3-II availability could be a therapeutic strategy against multiple disorders that stem from altered ERK activity (Martinez-Lopez et al., 2013).

The increased expression of Akt and ERK in the senescence conditioned media therefore suggests that these cells were able to proliferate even after DXR had been administered and potentially evades apoptotic cell death. However, contradictory to these above-mentioned results, the expression of senescence markers (like $p 16)$ was also increased $(p<0.05)$, which indicates that these cells were in a senescent state and are therefore not able to grow. However, these results could have a different outcome when various treatment durations of DXR are administered or if a greater percentage of senescence was induced in the fibroblast population.

Taken together, these results indicate that once senescence is induced in fibroblasts, tumour resistance is enhanced and the cancer cells have the ability to evade apoptosis. After investigating the effects of autophagy, the results suggest that autophagy contributes to cancer cell death in apoptosis and senescence conditioned media and does not contribute to 
treatment resistance. Importantly, the effects of autophagy are cell line specific and should be optimized for each cancer cell line. Autophagy inhibitors will therefore not improve the clinical response to chemotherapy in E0771 breast cancer cells, but the effects of necrosis or necroptosis can further be investigated to determine why the cancer cells are able to evade cell death. 


\section{Conclusion}

Senescent cells actively communicate with neighbouring cells and the TME, through the secretion of molecules that constitute the SASP (cytokines, chemokines, and growth factors). Senescent fibroblasts secrete certain factors that could promote the proliferation of cancer cells. The aim of this study was to assess the effects of conditioned media harvested from senescent and apoptotic fibroblasts on breast cancer cells. The results support the idea that fibroblasts can secrete paracrine factors that affects breast cancer growth and therapeutic resistance and that these effects differ when senescence and apoptosis is induced in the fibroblasts. Conditioned media from fibroblasts decreased cellular death in E0771 breast cancer cells treated with DXR, however, autophagy contributed to cancer cell death and other mechanisms of resistance must be investigated. We have therefore clearly demonstrated that fibroblasts are influenced by DXR treatment to secrete factors which make cancer cells less susceptible to cell death induced by chemotherapy. Our results also indicated for the first time that even a $56 \%$ senescent fibroblast population was able to significantly increase cell viability in E0771 cells after DXR treatment. 


\section{Summary of key findings}

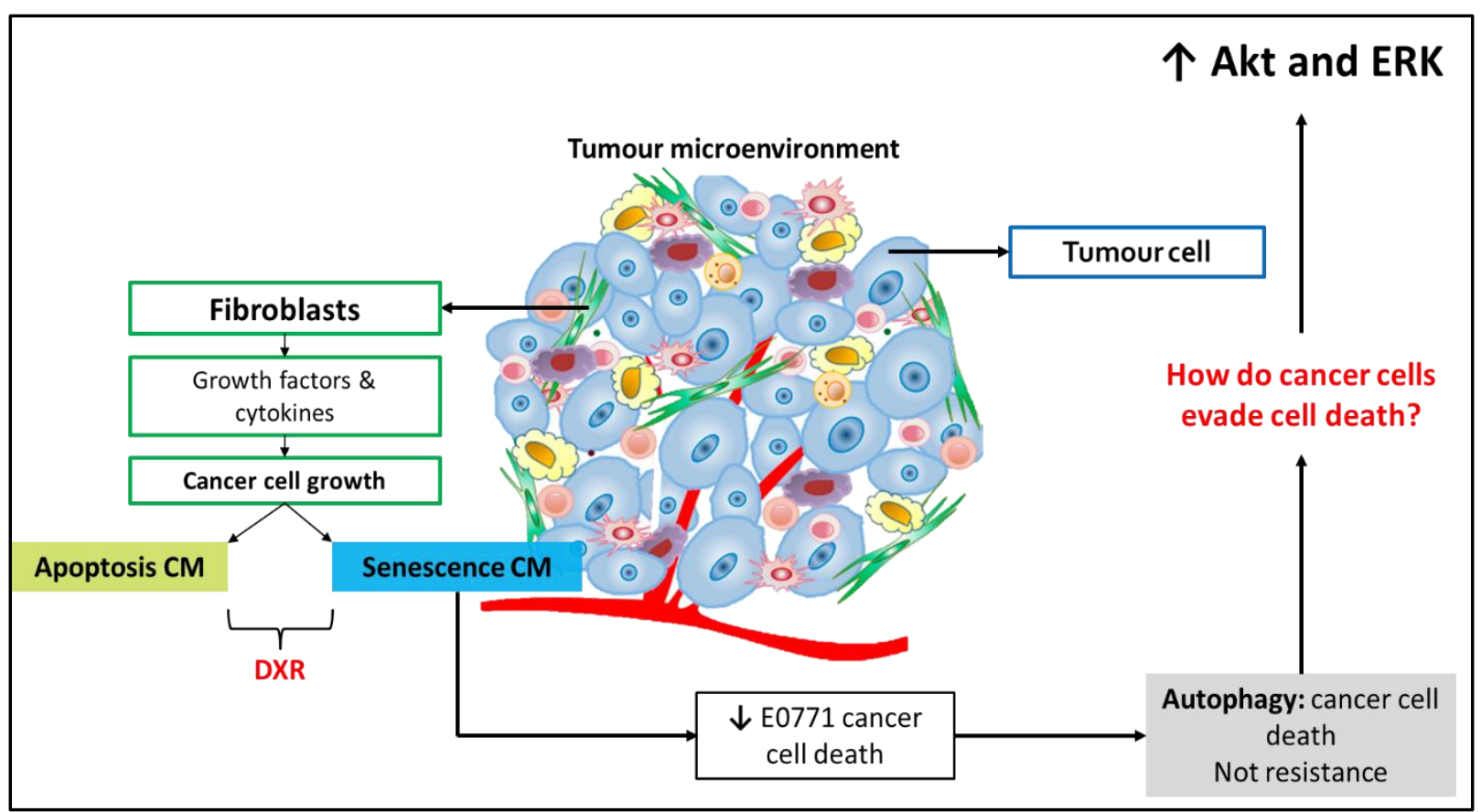

Figure 6.1: A summary of the key findings. Fibroblasts secrete growth factors and cytokines that affect cancer cell growth. Apoptosis and senescence was induced in a MEF population to generate conditioned media. Senescence CM allowed mouse breast cancer cells to evade apoptotic cell death even after DXR had been administered. Controversial to many studies, autophagy contributed to cancer cell death and not treatment resistance in apoptosis and senescence CM groups. The ability of E0771 cells to evade apoptotic cell death is attributed to significant increase in Akt and ERK expression, which enhances proliferation and suppresses the apoptotic pathway.

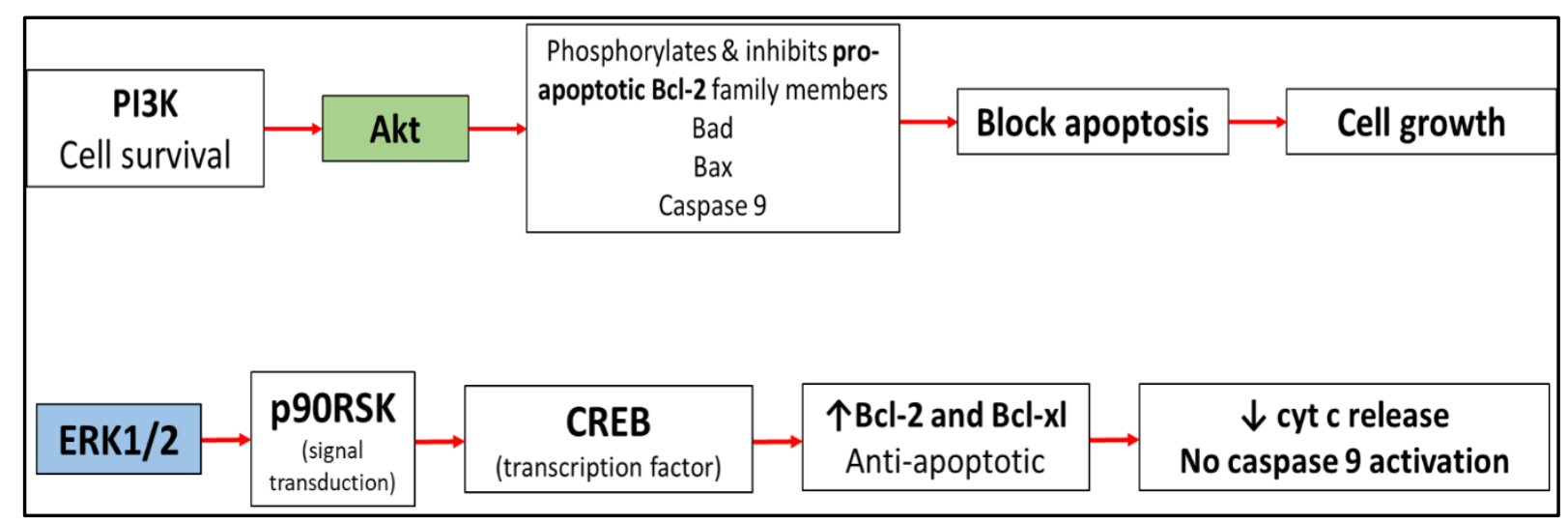

Figure 6.2: Akt and ERK contributes to cancer cell proliferation and DXR treatment resistance.

Akt inhibits the expression of pro-apoptotic family members to inhibit programmed cell death by blocking apoptosis. The PI3K/Akt pathway can induce pro-survival signalling in both cancerous and non- 
cancerous cells and could therefore explain why cell viability was increased in senescence CM. Furthermore, the activation of ERK increases the expression of anti-apoptotic molecules, which will contribute to the ability of cancer cells to evade cell death and could also explain why no caspase 9 activation was observed. 


\section{Limitations and future recommendations}

Ideally, the findings should be confirmed in an in vitro setting by increasing the sample size for better statistical power.

The protocol for senescence induction should be optimized so that $80 \%$ of the cell population is senescent. These results can then be compared with the current findings to determine if there are any variations.

The protocol for apoptosis induction in MEFs can further be optimized by altering treatment duration to determine whether there would be a different outcome. The expression of apoptotic markers could also be viewed at different time points to determine why there was minimal cleavage of certain caspase when DXR is known for its apoptosis induction.

To further support the results, it would have been optimal to determine the expression of senescent and proliferation markers present in the E0771 cells after fibroblast CM treatment at various time points or after a longer treatment duration to determine why the contradictory results are present.

Furthermore, western blot protocols could further be optimized specifically for E0771 cells. We were unable to obtain data for p21 in the E0771 cells.

Identifying the presence of certain cytokines in the collected CM, such as IL-6, secreted from senescent fibroblasts will add value to obtained results by potentially elucidating the molecular mechanisms for the observed results.

To determine why the cancer cells evade apoptotic cell death, the effects of necrosis should be investigated.

Once these results are repeated and confirmed, it could suggest that by specifically targeting cancer cells and not stromal fibroblasts with chemotherapeutic agents, patients with breast cancer could have a better response to treatment. 


\section{Bibliography}

Allinen, M., Beroukhim, R., Cai, L., Brennan, C., Lahti-domenici, J., Huang, H., Porter, D., Hu, M., et al. 2004. Molecular characterization of the tumour microenvironment in breast cancer. Cancer Cell:6;17-32.

Amaravadi R, Lippincott-Schwartz J, Xiao-Ming Yin, A. Weiss W, Takebe N, Timmer W, DiPaola R, Lotze $M$, and White E, 2011. Principles and Current Strategies for Targeting Autophagy for Cancer Treatment. Clinical Cancer Research: 17(4): 654-666.

Amornsupak, K., Insawang, T., Thuwajit, P., O-charoenrat, P. \& Eccles, S.A. 2014. Cancerassociated fibroblasts induce high mobility group box 1 and contribute to resistance to doxorubicin in breast cancer cells. BMC Cancer. 1-12.

Apte R, Dotan S, Elkabets M \& White M, 2006. The involvement of IL-1 in tumorigenesis, tumour invasiveness, metastasis and tumor-host interactions. Cancer Metastasis Reviews: 25:387-408.

Arpino, G., Bardou, V.J., Clark, G.M. \& Elledge, R.M. 2004. Infiltrating lobular carcinoma of the breast: Tumor characteristics and clinical outcome. Breast Cancer Research. 6(3):7-11.

Ashkenaz, A and Dixit. V, 1998. Death Receptors: Signalling and Modulation. Science:(281);1305-1309.

Ayala G, Thompson T \& Yang G, 2004. High levels of phosphorylated form of Akt-1 in prostate cancer and non-neoplastic prostate tissues are strong predictors of biochemical recurrence. Clinical Cancer Research:10;6572-8.

Aydinlik, S., Erkisa, M., Cevatemre, B., Sarimahmut, M., Dere, E., Ari, F. \& Ulukaya, E. 2017. Enhanced cytotoxic activity of doxorubicin through the inhibition of autophagy in triple negative breast cancer cell line. Biochimica et Biophysica Acta - General Subjects:1861(2);49-57.

Baselga, J, 2011. Targeting the Phosphoinositide-3(PI3) Kinase Pathway in Breast Cancer. The Oncologist:16;12-19.

Beause Âjour, C, Krtolica A, Galimi, F, \& Narita, M. 2003. Reversal of human cellular senescence: roles of the p53 and p16 pathways. The EMBO Journal:22(16):4212-4222.

Ben-Porath, I. \& Weinberg, R.A. 2005. The signals and pathways activating cellular senescence. International Journal of Biochemistry and Cell Biology:37(5);961-976. 
Bernard, A \& Klionsky, D. 2013. Autophagosome Formation: Tracing the Source. Developmental Cell:25(2):16-17.

Bradford, MM. 1976. A rapid and sensitive method for the quantitation of microgram quantities of protein utilizing the principle of protein-dye binding. Analytical Biochemistry: 72:248-54.

Brasier AR, 2010. The nuclear factor-kB-interleukin-6 signalling pathway mediating vascular inflammation. Cardiovascular Research:86; 211-218.

Bray, F., Ferlay, J., Soerjomataram, I. \& Siegel, R.L. 2013. Global Cancer Statistics 2018: GLOBOCAN Estimates of Incidence and Mortality Worldwide for 36 Cancers in 185 Countries. A cancer journal for clinicians:107(11);843-847.

Brech, A, Ahlquist, T, Lothe, R, \& Stenmark, H. 2009. Autophagy in tumour suppression and promotion. Molecular oncology:3;366-375.

Broustas, C \& Lieberman H. 2014. DNA Damage Response Genes and the Development of Cancer Metastasis. Radiation Research:181;111-130.

Brown, J \& Wouters B, 1999. Apoptosis, p53, and Tumour Cell Sensitivity to Anticancer Agents. Cancer Research:59;1391-1399.

Cagnol, S \& Chambard, J. 2009. ERK and cell death: Mechanisms of ERK-induced cell death - apoptosis, autophagy and senescence. The FEBS Journal: 277; 2-21.

Campisi, J. 1997. Aging and Cancer: The Double-Edged Sword of Replicative Senescence. Geriatric Bioscience:45;482-488.

Campisi, J. 2005. Senescent cells, Tumour suppression and Organismal Aging: Good citizens, bad neighbours. Cell:120;513-522.

Campisi, J. 2007. When bad things happen to good cells. Nature Reviews of molecular cell biology:8;729-740.

Caldwell, J.C. 2001. Population health in transition. Bulletin of the World Health Organization: 79(2):159-160.

Cantley, L. 2002. The Phosphoinositide 3-Kinase Pathway. Science:296;1655-1700.

Capparelli C, Guido C, Whitaker-Menezes D, Bonuccelli G, Balliet R, Pestell T, Goldberg F, Pestell RG, Howell A, Sneddon S, Birbe R, Tsirigos A, Martinez-Outschoorn U, Sotgia F \&. Lisanti MP. 2012. Autophagy and senescence in cancer-associated fibroblasts metabolically supports tumour growth and metastasis, via glycolysis and ketone production. Cell Cycle:11(12);2285-2303. 
Cardone, MH, Roy, N, Stennicke, HR, Salvesen, GS, Franke, TF, Stanbridge, E, Frisch, S \& Reed, C. 1998. Regulation of cell death protease caspase-9 by phosphorylation. Science:282;1318-1321.

Carpenter, AE, Jones, TR, Lamprecht, MR, Clarke, C, Kang, IH, Friman, O, Guertin, DA, Chang, JH, Lindquist, RA, Moffat, J, Golland, P \& Sabatini, DM. 2006. Cell Profiler: image analysis software for identifying and quantifying cell phenotypes. Genome Biology:7;100.

Castellano and Downward J. 2011. RAS Interaction with PI3K: More Than Just Another Effector Pathway. Genes \& Cancer.2(3);261 -274.

Cauchi, J.P., Camilleri, L. \& Scerri, C. 2016. Environmental and lifestyle risk factors of breast cancer in Malta-a retrospective case-control study. EPMA Journal:7(1):1-10.

Chang, M. 2012. Tamoxifen resistance in breast cancer. Biomolecules and Therapeutics:20(3):256-267.

Chang, B., Swift, M.E., Shen, M., Fang, J., Broude, E. V \& Roninson, I.B. 2002. Molecular determinants of terminal growth arrest induced in tumour cells by a chemotherapeutic agent. PLOS ONE:99(1).

Chinnaiyan AM. 1999. The Apoptosome: Heart and Soul of the Cell Death Machine. Neoplasia:1(1);5-15.

Chua BT, Gallego-Ortega D, Ramirez A, Ulrich A \& Downword J. 2009. Regulation of Akt in breast carcinoma cells. Molecular Cancer:8;131.

Colell A, 2007. GAPDH and Autophagy preserve survival after apoptotic cytochrome $C$ release in the absence of caspase activation. Cell:129;983-997.

Coley, HM, Amos, WB, Twentyman, PR \& Workman, P.1993. Examination by laser scanning confocal fluorescence imaging microscopy of the subcellular localisation of anthracyclines in parent and multidrug resistant cell lines. British Journal of Cancer:67;1316-23.

Conze D, Weiss L, Regen P, Bhushan A, Weaver D, Johnson P, and Rinco'n M. 2001. Autocrine Production of Interleukin 6 Causes Multidrug Resistance in Breast Cancer Cells. Cancer Research:61; 8851-8858.

Coppé, J.-P., Patil, C.K., Rodier, F., Sun, Y., Muñoz, D.P., Goldstein, J., Nelson, P.S \& Desprez, P.-Y., 2008. Senescence-Associated Secretory Phenotypes Reveal CellNonautonomous Functions of Oncogenic RAS and the p53 Tumor Suppressor. PLoS Biology:6(12):301. 
Cory, S \& Adams JM. 2002. THE BCL2 FAMILY: Regulators of the cellular life or death switch. Nature Reviews:2;647-654.

Coussens, LM \& Werb, Z. 2010. Inflammation and cancer. Nature: 420(6917): 860-867.

Cruet-Hennequart, S \& Prendergast, A. 2012. Doxorubicin induces the DNA damage response in cultured human mesenchymal stem cells. International Journal of Hematology:96;649-656.

Das G, Shravage BV and Baehrecke EH. 2012. Regulation and Function of Autophagy during Cell Survival and Cell Death. Cold Spring Harb Perspect Biology:4.

Davalos, A.R., Coppe, J., Campisi, J. \& Desprez, P. 2010. Senescent cells as a source of inflammatory factors for tumor progression. Cancer Metastasis Reviews:29;273-283.

Degenhardt, K, Mathew, R, Beaudoin, B, Bray, K, Anderson, D, Chen G \& White, E. 2006. Autophagy promotes tumor cell survival and restricts necrosis, inflammation, and tumorigenesis. Cancer Cell:(1): 51-64.

Demaria, M, O'Leary, MN, Chang, J, Shao, L, Liu, S, Alimirah, F, Koenig, K, Le, C, Mitin N, Deal, AM, Alston, S, Academia, EC, Kilmarx, S, Valdovino,S A, Wang, B, de Bruin, A, Kennedy, BK, Melov, S, Zhou, D, Sharpless, NE, Muss, H \& Campisi J. 2016. Cellular Senescence Promotes Adverse Effects of Chemotherapy and Cancer Relapse. Cancer Discovery:7(2);165-176.

Desantis, C.E., Fedewa, S.A., Sauer, A.G., Kramer, J.L., Smith, R.A. \& Jemal, A, 2016. Breast Cancer Statistics: Convergence of Incidence Rates Between Black and White Women. $A$ cancer journal for clinicians:66(1):31-42.

Doonan, F \& Cotter T. 2007. Morphological assessement of apoptosis. Methods:44;200-204.

Downward, J, 2004. "PI 3-kinase, Akt and cell survival," Seminars in Cell and Developmental Biology:15(2); 177-182.

Drescher S, Schaeffeler E, Monika Hitzl M, Hofmann U, Brinkmann U \& Fromm M. 2002. MDR1 gene polymorphisms and disposition of the P-glycoprotein substrate fexofenadine. Clinical Pharmacology:53;526-534.

Duque GA \& Descoteaux A. 2014. Macrophage cytokines. Frontiers in Immunology:5;491.

Ellis, P.A., Saccani-Jotti, G., Clarke, R., Johnston, S.R.D., Anderson, E., Howell, A., A'Hern, R., Salter, J, 1997. Induction of apoptosis by tamoxifen and ICI 182780 in primary breast cancer. International Journal of Cancer:72(4):608-613. 
Elmore, S. 2007. Apoptosis: A Review of Programmed Cell Death. Toxicologic Pathology:35(4):495-516.

Esclatine A, Chaumorcel M \& Codogeno P. 2009. Macroautophagy Signaling and Regulation. Current Topics in Microbiology and Immunology:335;33.

Esquivel-Vela'zquez, M Ostoa-Saloma, P, Palacios-Arreola, MI, Nava-Castro, KE, \& MoralesMontor J. 2015. The Role of Cytokines in Breast Cancer Development and Progression. Journal of Interferon \& Cytokine Research:35(1).

Etemadmoghadam, D, Weir, BA, Au-Yeung, G, Alsop, K, Mitchell, G \&George, J. 2013. Synthetic lethality between CCNE1 amplification and loss of BRCA1. PNAS:110(48); 1948919494.

Ewald JA, Wilding G, \& Jarrard D. 2010. Therapy-Induced Senescence in Cancer. Journal for Clinicians:102(20):1536-1545.

Ferrel, E \& Bhatt, R, 1997. Mechanistic Studies of the Dual Phosphorylation of Mitogenactivated Protein Kinase. Journal of Biological Chemistry: 272.

Fiaschi, T \& Chiarugi, P. 2012. Oxidative Stress, Tumour Microenvironment, and Metabolic Reprogramming: A Diabolic Liaison. International Journal of Cell Biology.

Forouzanfar, M.H., Foreman, K.J., Delossantos, A.M., Lozano, R., Lopez, A.D., Murray, C.J.L. \& Naghavi, M, 2011. Breast and cervical cancer in 187 countries between 1980 and 2010: A systematic analysis. The Lancet:378(9801):1461-1484.

Frankel, LB and Lund, A. 2018. MicroRNA regulation of autophagy. Carcinogenesis:33(11);2018-2025.

Fung, C, Lock, R, Goa, S, Salas, E \& Debnath, J. 2007. Induction of Autophagy during Extracellular Matrix Detachment Promotes Cell Survival. Molecular Biology of the Cell:19;797806.

Galluzi et al., 2018. Molecular mechanisms of cell death: recommendations of the Nomenclature Committee on Cell Death 2018. Cell Death \& Differentiation: 25:486-541.

Garnett, MJ \& Marais, R. 2004. Guilty as charged: B-RAF is a human oncogene. Cancer Cell:6;313.

Gewirtz, DA. 1999. A Critical Evaluation of the Mechanisms of Action Proposed for the Antitumor Effects of the Anthracycline Antibiotics Adriamycin and Daunorubicin. Biochemical Pharmacology: 57; 727. 
Gewirtz, DA. 2007. Autophagy as a Mechanism of Radiation Sensitization in Breast Tumour Cells. Autophagy:3(3);249-250.

Gewirtz, DA, Holt, S \& Elmore, LW. 2009. Accelerated Senescence. Biochemical Pharmacology:76;947-957.

Gewirtz, DA. 2009. Autophagy, senescence and tumour dormancy in cancer therapy. Autophagy:5(8);1232.

Gewirtz, DA. 2016. The challenge of developing autophagy inhibition as a therapeutic strategy. Cancer Research: 76(19): 5610-561.

Giordano, S.H., Cohen, D.S., Buzdar, A.U., Perkins, G. \& Hortobagyi, G.N, 2004. Breast carcinoma in men: A population-based study. Cancer:101(1):51-57.

Goehe, RW, Di, X, Sharma, K, Bristol, M, Henderson, S, Valerie, K \& Gewirtz, DA. 2012. The Autophagy-Senescence Connection in Chemotherapy: Must Tumour Cells (Self) Eat Before They Sleep? The Journal of Pharmacology and experimental Therapeutics:343(3);763-771.

Gudaviciene, D., Steponaviciene, L., Meskauskas, R., Smailyte, G. \& Aleknavicius, E. 2015. Rare types of breast carcinoma. Open Medicine:10(1);92-96.

Guo, J, Chen, H, Mathew, R, Fan, J, Stroheker, A, Chen, G, Lemons, J \& White, E. 2011. Activated Ras requires autophagy to maintain oxidative metabolism and tumorigenesis. Genes and Development:25;460-470.

Hanahan, D \& Weinberg, RA. 2000. The Hallmarks of Cancer. Cell:10;57-70.

Harper, JW \& Elledge, SJ. 2007. The DNA damage response. Molecular Cell:28;739.

Harris, S.L. \& Levine, A.J. 2005. The p53 pathway: positive and negative feedback loops. Oncogene:24;2899-2908.

He, C \& Klionsky, DJ. 2009. Regulation Mechanisms and Signalling Pathways of Autophagy. Annual review of Genetics:43;67-93.

Hersey, P \& Zhang, D. 2003. Overcoming Resistance of Cancer Cells to Apoptosis. Journal of cellular Physiology: 196:9-18.

Hill, MM, Adrain, C, Duriez, PJ \& Martin, SJ. 2004. Analysis of the composition, assembly kinetics and activity of native Apaf-1 apoptosomes. The EMBO Journal:23;2134-2145.

Hindenburg, AA, Gervasoni, JE, Krishna, S, Stewart, VJ, Rosado M \& Lutzky J. 1989. 
Intracellular distribution and pharmacokinetics of daunorubicin in anthracycline-sensitive and -resistant HL-60 cells. Cancer Research:49;4607-14.

Housman, G., Byler, S., Heerboth, S., Lapinska, K., Longacre, M., Snyder, N. \& Sarkar, S. 2014. Drug resistance in cancer: An overview. Cancers:6(3);1769-1792.

Hu, M \& Polyak, K. 2008. Molecular characterization of the tumour microenvironment in breast cancer. European Journal of Cancer:44(18): 2760-2765.

Huang, Z, Zhou, L, Chen, Z, Nice, EC \& Huang, C. 2016. Stress management by autophagy: Implications for chemoresistance. International Journal of Cancer:139;23-32.

Jean-Philippe Coppé, Pierre-Yves Desprez, Ana Krtolica \& J.C. 2014. The SenescenceAssociated Secretory Phenotype: The Dark Side of Tumour Suppression. Annual Review of Pathology:5;99-118.

Jiang, P \& Mizushima, N. 2014. Autophagy and human diseases. Cell Research:24;69-79.

Kaltschmidt, B, Hofmann, T, Hehner, S, Droge, W \& Schmitz, ML. 2000. The pro- or antiapoptotic function of NF-kB is determined by the nature of the apoptotic stimulus. European Journal of Biochemistry: 267;3828-3835.

Kandel, ES, Skeen, J, Majewski, N, Pandolfi, P, Feliciano, S \& Hay, N. 2002. Activation of Akt/Protein Kinase B Overcomes a G2/M Cell Cycle Checkpoint Induced by DNA Damage. Molecular and cellular Biology:22(22);7831-7841.

Karantza, V, Patel, SKravchuk, O, Chen, G, Mathew, R, Jin, S \& White, E. 2007. Autophagy mitigates metabolic stress and genome damage in mammary tumorigenesis. Genes and Development 21:1621-1635.

Karar, J \& Maity, A. 2011. PI3K/Akt/mTOR pathway is Angiogenesis. Molecular Neuroscience:4; 51.

Kelloff, G.J., Sigman, C.C., Johnson, K.M., Boone, C.W., Greenwald, P., Crowell, J. a, Hawk, E.T. \& Doody, L, 2000. Perspectives on Surrogate End Points in the Development of Drugs that Reduce the Risk of Cancer. Prevention:9;127-137.

Kerr, JF \& Currie, AR. 1972. APOPTOSIS: A basic biological phenomenon with wide ranging implications in tissue kinetics. British journal of Cancer:26;239.

Kim, H, Shah, M, Tu, H, Jeffers, JR, Hsieh, JD \& Cheng, E. Hierarchical regulation of mitochondrion-dependent apoptosis by BCL-2 subfamilies. Nature:8(12);1349. 
Kirisako, T, Baba, M, Ishihara, N \& Oshumi, Y. 1999. Formation Process of Autophagosome Is Traced with Apg8/Aut7p in Yeast. The Journal of Cell Biology:147;436-440.

Kischkel, FC, Hellbardt, S, Behrmann, I, Germer, R \& Peter, ME. 1995. Cytotoxicity-dependent APO-1 (Fas/CD95) associated proteins form a death-inducing signaling complex (DISC) with the receptor. The EMBO Journal:14(22);5579-5588.

Klionsky, DJ. The molecular machinery of autophagy: unanswered questions. Journal of Cell Science:1(118);7-18.

Knu "pfer, H \& Preis, R. 2007. Significance of interleukin-6 (IL-6) in breast cancer (review). Breast Cancer research and treatment:102;129-135.

Koleini, N \& Kardami, E. 2017. Autophagy and mitophagy in the context of doxorubicininduced cardiotoxicity. Oncotarget:8(28): 46663-46680.

Komatsu, M, Waguri, S, Koike, M, Sou, Y, Warabi, E, Yue, Z \& Tanaka, K. 2007. Homeostatic levels of p62 control cytoplasmic inclusion body formation in autophagy-deficient mice. Cell:131;1149-1163.

Kondo, Y \& Kondo S. 2006. Autophagy and cancer therapy. Autophagy:2;85-90.

Krtolica, A., Parrinello, S., Lockett, S., Desprez, P.-Y. \& Campisi, J, 2001. Senescent fibroblasts promote epithelial cell growth and tumorigenesis: A link between cancer and aging. Proceedings of the National Academy of Sciences:98(21);12072-12077.

Kucerova, L., Skolekova, S., Matuskova, M., Bohac, M. \& Kozovska, Z, 2013. Altered features and increased chemosensitivity of human breast cancer cells mediated by adipose tissuederived mesenchymal stromal cells. BMC Cancer.

Kumar, A, Rajendran, V \& Purohit, R. 2013. AKT Kinase Pathway: A Leading Target in Cancer Research. The Scientific World Journal:2013;1-6.

Kumar, P. \& Aggarwal, R. 2008. An overview of triple-negative breast cancer. Archives of Gynaecology and Obstetrics:293(2):247-269.

Kumar, P \& Aggarwal, R. 2016. An overview of triple-negative breast cancer. Archives of Gynaecology and Obstetrics:293;247-269.

Kurz, D.J., Decary, S., Hong, Y. \& Erusalimsky, J.D. 2000. Senescence-associated (beta)galactosidase reflects an increase in lysosomal mass during replicative ageing of human endothelial cells. Journal of cell science:113(20);3613-22. 
Kuwabara, I, Yang, R, Schuler, M, Green, DR, Liu, F. 2002. Galectin-7 (PIG1) Exhibits Proapoptotic Function through JNK Activation and Mitochondrial Cytochrome c Release. THE JOURNAL OF BIOLOGICAL CHEMISTRY:277(5);2487-3494.

Kwon, Y, Kim, J, Jeoung, JA \& Kang, C. 2017. Autophagy Is Pro-Senescence When Seen in Close-Up, but Anti-Senescence in Long-Shot. Cell:40(9);607-612.

Lawrence, T. 2009. The Nuclear Factor NF-kB Pathway in Inflammation. Cold Spring Harbour Perspectives in Biology:1;1651.

Lei, M. 2005. The MCM complex: its role in DNA replication and implications for cancer therapy. Current Cancer Drug Targets:5(5);365-80.

Leicht, DT, Balan, V, Kaplun, A, Singh-Gupta, V, Dobson, M \& Tzivion, G. 2007. Raf Kinases: Function, Regulation and Role in Human Cancer. Biochimica et Biophysica Acta:1773(8);1196-1212.

Levine, B \& Klionsky, DJ. 2004. Development by Self-Digestion: Molecular Mechanisms and Biological Functions of Autophagy. Developmental Cell:6;463-477.

Levine, B \& Kroemer, G. 2007. Autophagy in the Pathogenesis of Disease. Cell:132(1);27-42. Lewis, A \& Alexander, HR. 2006. Interleukin-1 and cancer progression. Journal of Translational Medicine:4(48).

$\mathrm{Li}, \mathrm{H}$, Zhu, H, Xu, C \& Yuan, J. 1998. Cleavage of BID by Caspase 8 Mediates the Mitochondrial Damage in the Fas Pathway of Apoptosis. Cell:94;491-501.

Li, Cl, Malone, K, Porter, PI, Daling, J. 2003. Relationship between long durations and different regimens of hormone therapy and risk of breast cancer. Journal of the American Medical Association:289;3254-3263.

Li, X, Lu, Y, Liang, K, Liu, B \& Fan, Z. 2005. Differential responses to doxorubicin-induced phosphorylation and activation of Akt in human breast cancer cells. Breast Cancer Research:7(5);590-596.

Li, Cl., Uribe, D. \& Daling, JR. 2005. Clinical characteristics of different histologic types of breast cancer. British Journal of Cancer:93(9):1046-1052.

Li, H, Fan, X \& Houghton, J. 2007. Tumour Microenvironment: The Role of the Tumour Stroma in Cancer. Journal of Cellular Biochemistry:101;805-815.

Liu, P, Cheng, H, Roberts, TM, Zhao, J. 2009. Targeting the phosphoinositide 3-kinase (PI3K) pathway in cancer. Nature Reviews Drug Discovery:8(8);627-644. 
Locksley, RM, Killeen, N, Lenardo, MJ. 2001. The TNF and TNF Receptor Superfamilies: Integrating Mammalian Biology. Cell:104;487-501.

Lovitt, CJ, Shelper, TB \& Avery, AM. 2018. Doxorubicin resistance in breast cancer cells is mediated by extracellular matrix proteins. BMC cancer:18(1);41.

Lujambio, A. 2016. To clear, or not to clear (senescent cells)? That is the question. Bioessays:87-95.

Lum, JJ, Bauer, DE, Kong, M, Harris, M \& Thompson, TB. 2005. Growth Factor Regulation of Autophagy and Cell Survival in the Absence of Apoptosis. Cell:120;237-248.

Madrid, LV, Wang, C, Guttridge, C, Baldwin, AS \& Mayo, M. 2000. Akt Suppresses Apoptosis by Stimulating the Transactivation Potential of the RelA/p65 Subunit of NF-kB. MOLECULAR AND CELLULAR BIOLOGY:20(5);1626-1638.

Maehama, T \& Dixon, JE. 1998. The Tumour Suppressor, PTEN/ MMAC1, Dephosphorylates the Lipid Second Messenger, Phosphatidylinositol 3,4,5-Trisphosphate. THE JOURNAL OF BIOLOGICAL CHEMISTRY:272(23); 13375-13378.

Mah, L \& Ryan, K. 2012. Autophagy and Cancer. Cold Spring Harbour Perspectives in Biology:4;8821.

Maiuri, MC, Zalckvar, E, Kimchi, A \& Kroemer, G. 2007. Self-eating and self-killing: crosstalk between autophagy and apoptosis. Nature Reviews Molecular Cell Biology:8;741-52.

Mao, Y, Keller, ET, Garfield, D, Shen, K \& Wang, J. 2013. Stroma Cells in Tumour Microenvironment and Breast Cancer. Cancer Metastasis:30;305-312.

Martinez-Lopez, N, Mishall, P \& Singh, R, 2013. Autophagy proteins regulate ERK phosphorylation. Nature Communications:4:2799.

Martinez-Outschoorn UE, Pavlides S, Whitaker-Menezes D, Daumer KM, Milliman JN, Chiavarina B, Migneco G, Witkiewicz AK, Martinez-Cantarin MP, Flomenberg N, Howell A, Pestell RG, Lisanti MP \& Sotgia F, 2010. Tumour cells induce the cancer associated fibroblast phenotype via caveolin-1 degradation: implications for breast cancer and DCIS therapy with autophagy inhibitors. Cell Cycle:15;9(12):2423-33.

Maycotte, P \& Thorburn, A. 2011. Autophagy and cancer therapy. Cancer Biology \& Therapy: $11:(2) ; 127-137$. 
McCubrey, J, Steelman, L, Chappell, W, Abrams, SL, Wong, E, Chang, F, Terrian, DM, Libra, M \& Franklin, RA. 2007. Roles of the Raf/MEK/ERK pathway in cell growth, malignant transformation and drug resistance: Biochim Biophys Acta:1773(8);1263-1284.

McCubrey, J, Steelman, L, Chappell, W, Abrams, SL, Wong, E, Chang, F, Terrian, DM, Libra, M, Franklin, RA \& Martelli, AM. 2012. Ras/Raf/MEK/ERK and PI3K/PTEN/Akt/mTOR Cascade Inhibitors: How Mutations Can Result in Therapy Resistance and How to Overcome Resistance. Oncotarget:3(10);1068-1111.

Meffert, MK \& Wiltgen, B. 2003. NFkB functions in synaptic signalling and behaviour. Nature:6(10):1072.

Michaud. M, Martins, I, Sukkarwalla, A, Ma, Y, Pellegatti, P, Shen, S, Menger, L \& Kroemer, G. 2011. Autophagy-Dependent Anticancer Immune Responses Induced by Chemotherapeutic Agents in Mice. Science:334;1573.

Mishra, P, Banerjee, D \& Ben-Baruch, A. 2010. Chemokines at the crossroads of tumorfibroblast interactions that promote malignancy. Journal of Leukocyte Biology:89;31-40.

Mizutani, H., Oikawa, S., Hiraku, Y., Murata, M., Kojima, M. \& Kawanishi, S. 2003. Distinct mechanisms of site-specific oxidative DNA damage by doxorubicin in the presence of copper (II) and NADPH-cytochrome P450 reductase. Cancer Science:94(8);686-691.

Momparler, RL, Karon, M \& Siegel SE, 1976. Effect of Adriamycin on DNA, RNA, and protein synthesis in cell-free systems and intact cells. Cancer Research: 36:2891-5.

Montagut, C \& Settleman, J. 2009. Targeting the Raf-Mek-ERK pathway in cancer. Cancer letters:283;125-134.

Mortusewicz, O, Ame, J, V Schreiber, V \& Leonhardt, H. 2007. Feedback-regulated poly(ADPribosyl)ation by PARP-1 is required for rapid response to DNA damage in living cells. Nucleic Acids Research:35(22); 7665-7675.

Moscat, J \& Diaz-Meco, DT. 2009. p62 at the Crossroads of Autophagy, Apoptosis, and Cancer. Cell:137(6);1001-1004.

Mosmann, T. 1983. Rapid Colorimetric Assay for Cellular Growth and Survival: Application to Proliferation and Cytotoxicity Assays. Journal of Immunological Methods:65;55-63.

Mutgan A, Besikcioglu, H, Wang, S, Friess, H, Ceyhan, G, \& Demir, IE.2018. Insulin/IGFdriven cancer cell-stroma crosstalk as a novel therapeutic target in pancreatic cancer. Molecular cancer.17;66. 
Myung, J, Kim, KB \& Crews, CM. 2001. The Ubiquitin-Proteasome Pathway and Proteasome Inhibitors. Medicinal Research Reviews:21(4);245-273.

Narita, M., Narita, M., Krizhanovsky, V., Nuñez, S., Chicas, A., Hearn, S.A., Myers, M.P. \& Lowe, S.W. 2006. A Novel Role for High-Mobility Group A Proteins in Cellular Senescence and Heterochromatin Formation. Cell:126(3);503-514.

Ngabire, D \& Kim, G. 2017. Autophagy and Inflammatory Response in the Tumour Microenvironment. International Journal of Molecular Sciences:18;2016.

Nounou, M.I., Elamrawy, F., Ahmed, N., Abdelraouf, K., Goda, S. \& Syed-Sha-Qhattal, H. 2015. Breast cancer: Conventional diagnosis and treatment modalities and recent patents and technologies supplementary issue: Targeted therapies in breast cancer treatment. Breast Cancer: Basic and Clinical Research:9;17-34.

Omran AR. 1971. The epidemiologic transition: a theory of the epidemiology of population change. Milbank Memorial Fund Quarterly:29: 509-538.

Ortiz-Montero, P, Londoño-Vallejo, A \& Vernot, J. 2017. Senescence-associated IL-6 and IL8 cytokines induce a self- and crossreinforced senescence/inflammatory milieu strengthening tumorigenic capabilities in the MCF-7 breast cancer cell line. Cell Communication and Signaling:15(17).

Pankiv, S, Clausen, TH, Lamark, T, Brech, A, Bruun, JA, \& Outzen, H. 2007. p62/SQSTM1 binds directly to Atg8/LC3 to facilitate degradation of ubiquitinated protein aggregates by autophagy. The Journal of Biological Chemistry:282;24131-45.

Pareja, F., Geyer, F.C., Marchiò, C., Burke, K.A., Weigelt, B. \& Reis-Filho, J.S. 2016. Triplenegative breast cancer: the importance of molecular and histologic subtyping, and recognition of low-grade variants. Breast Cancer:2(1);16036.

Parton, M., Dowsett, M., Smith, I. \& Smith, I. 2001. Studies of apoptosis in breast cancer. British Medical Journal:322;1528-1532.

Peiris-pagès, M., Sotgia, F. \& Lisanti, M.P. 2015. Chemotherapy induces the cancerassociated fibroblast phenotype, activating paracrine Hedgehog-GLI signalling in breast cancer cells. Oncotarget:6(13);10728-10745.

Peter, ME \& Krammer, PH. 1998. Mechanisms of CD95 (APO-1/Fas)-mediated apoptosis. Current Opinion in Immunology:10;545-551. 
Rampurwala, M.M., Rocque, G.B. \& Burkard, M.E. 2014. Breast Cancer: Basic and Clinical Research Update on Adjuvant Chemotherapy for Early Breast Cancer. Breast Cancer and Clinical Research:8;125-133.

Rathore, R, McCallum, J, Varghese, E,Florea, A \& Busselberg, D. 2017. Overcoming chemotherapy drug resistance by targeting inhibitors of apoptosis proteins (IAPs). Apoptosis:22;898-919.

Ricci, J, Munoz, C, Fitzgerald, P, Perkins, GA, Yadava, N, Ellisman, MH, Green, DR. 2004. Disruption of Mitochondrial Function during Apoptosis Is Mediated by Caspase Cleavage of the p75 Subunit of Complex I of the Electron Transport Chain. Cell: 117;773-786.

Rivankar, S, 2014. An overview of doxorubicin formulations in cancer therapy. Journal of Cancer research and Therapeutics: 10(4): 853-858.

Robinson, MJ \& Cobb, MH. 1997. Mitogen-activated protein kinase pathways. Current Opinion in Cell Biology:9;180-186.

Rock, KL, Gramm, C, Rothstein, L, Clark, K, Dick, L, Hwang, D \& Goldberg, AL. 1994. Inhibitors of the Proteasome Block the Degradation of Most Cell Proteins and the Generation of Peptides Presented on MHC Class I Molecules. Cell:78;761-771.

Rodriguez-Enriquez, S, He, L \& Lemasters, JJ. 2004. Role of mitochondrial permeability transition pores in mitochondrial autophagy. The International Journal of Biochemistry \& Cell Biology:36;2463-72.

Romagosa, C, Simonetti, S, López-Vicente, L, Mazo, A, Lleonart, ME, Castellv, I \& Ramon y Cajal, S. 2011. p16(Ink4a) overexpression in cancer: a tumour suppressor gene associated with senescence and high-grade tumours. Oncogene:5;30(18):2087-97.

Roninson, I.B. 2003. Tumour Cell Senescence in Cancer Treatment. Cancer Research:63(11): 2705-2715.

Ryan, BM, McManus, MO, Daly, JS, Kneeling, PW, Weir, DG, Lefort, F \& Kelleher, D. 2000. Serum Mutant K-ras in the Colorectal Adenoma-to-Carcinoma Sequence. Annals New York Academy of Sciences:29-31.

Sadeghi, N \& Gerber, DE. 2012. Targeting the PI3K pathway for cancer therapy. Future Medicinal Chemistry:4(9);1153-1169.

Schindelin, J, Arganda-Carreras, I \& Frise, E. 2012. "Fiji: an open-source platform for biological-image analysis". Nature methods: 9(7);676-682. 
Sebolt-Leopold, JS. 2000. Development of anticancer drugs targeting the MAP kinase pathway. Oncogene:19;6594-6599.

Serrano, M, Lin, AW, McCurrach, ME, Beach, D \& Lowe, SW. 1997. Oncogenic ras Provokes Premature Cell Senescence Associated with Accumulation of p53 and p16INK4a. Cell:88;593-602.

Shalini, S, Dorstyn, L, Dawar, S \& Kuma, S. 2015. Old, new and emerging functions of caspases. Cell Death and Differentiation:22;526-539.

Sharma G, Sanadya R, Sharma P, 2010. Various types and management of breast cancer: an overview. Journal of Advanced Pharmaceutical Technology \& Research:1(2):109-126.

Shen, C, Chen, H, Lin, M, Li, F, Chang, C, Kuo, M, Settleman, J \& Chen, R. 2008. Breast Tumor Kinase Phosphorylates p190RhoGAP to Regulate Rho and Ras and Promote Breast Carcinoma Growth, Migration, and Invasion. Cancer Research:68(19);7779.

Sher, CJ \& McCormick, F. 2002. The RB and p53 pathways in cancer. Cancer cell:2;103-109. Shields, JM, Pruitt, K, McFall, A, Shaub, A \& Der DJ. 2000. Cell Biology:10;147-155.

Siegel, R.L., Miller, K.D. \& Jemal, A. 2018. Cancer Statistics, 2018. Cancer journal for clinicians:67(1);7-30.

Sims, A. 2007. Origins of breast cancer subtypes. Nature:4(9).

Sirbu, BM \& Cortez, D. 2013. DNA Damage Response: Three Levels of DNA Repair Regulation. Cold Spring Harbour Perspectives in Biology:5.

South African National Cancer Registry. Cancer in South Africa. 2013. Johannesburg. www.ncr.ac.za. Accessed $\{20$ March 2018\}. http://www.nicd.ac.za/index.php/centres/nationalcancer-registry/cancer-statistics/.

Stansel, RM, Subramanian, D \& Griffith, JD. 2002. p53 Binds Telomeric Single Strand Overhangs and t-Loop Junctions in Vitro. The Journal of Biological Chemistry:277(14); 1162511628.

Steelman, LS, Pohnert, SC, Shelton, JG, Franklin, RA, Bertrand, FE \& McCubrey, JA. 2004. JAK/STAT, Raf/MEK/ERK, PI3K/Akt and BCR-ABL in cell cycle progression and leukemogenesis. Leukemia:18;189-218.

Stein, GH, Drullinger, LF, Soulard, A \& Dulic, V. 1999. Differential Roles for Cyclin-Dependent Kinase Inhibitors p21 and p16 in the Mechanisms of Senescence and Differentiation in Human Fibroblasts. Molecular and cellular Biology; 2109-2117. 
Stemke-Hale, K, Gonzalez-Angulo, A, Lluch, A, Neve, RM Kuo, W, Davies, M, Carey, M, Hu, Z, Guan, Y Sahin, A, Symmans, WF, Pusztai, L, Nolden, LK, Horlings, H, Berns, K, Hung, M, van de Vijver, M, Valero V, \& Hennessy, BT. 2008. An Integrative Genomic and Proteomic Analysis of PIK3CA, PTEN, and AKT Mutations in Breast Cancer. Cancer Research:68(15);6084-6091.

Suliman, A, Lam, A, Datta, R \& Srivastava, RK. 2001. Intracellular mechanisms of TRAIL: apoptosis through mitochondrial-dependent and -independent pathways. Oncogene:20;21222133.

Suman, S, Das, TP, Reddy, R, Nyakeriga, AM, Luevano, JE, Konwar, D \& Damodaran, P. 2014. The pro-apoptotic role of autophagy in breast cancer. British Journal of Cancer:111;309-317.

Taatjes,DJ, Gaudiano, G, Resing, K \& Koch, TH. 1997. Redox Pathway Leading to the Alkylation of DNA by the Anthracycline, Antitumor Drugs Adriamycin and Daunomycin. Journal of Medicinal Chemistry:40;1276-1286.

Taherian, A \& Mazoochi, T. 2012. Different Expression of Extracellular Signal-Regulated Kinases (ERK) 1/2 and Phospho-Erk Proteins in MBA-MB-231 and MCF-7 Cells after Chemotherapy with Doxorubicin or Docetaxel. Iranian Journal of Basic Medical Sciences:15(1);669.

Tanida, I, Ueno, T \& Kominami, E. 2008. LC3 and Autophagy. Methods in Molecular Biology:445;77-88.

Torre, L.A., Islami, F., Siegel, R.L., Ward, E.M. \& Jemal, A. 2017. Global cancer in women: Burden and trends. Cancer Epidemiology Biomarkers and Prevention:26(4);444-457.

Vassilev, A \& DePhamphilis, ML. 2017. Links between DNA Replication, Stem Cells and Cancer. Genes: 8(2);45.

Vivanco, I \& Sawyers, CL, 2002. The phosphatidylinositol 3-Kinase-AKT pathway in human cancer. Nature Reviews:2: 489-501.

Waite, KA \& Eng, C. 2002. Protean PTEN: Form and Function. The American Journal of Human Genetics:70;829-844.

Wang, C \& Youle, RJ. 2009. The Role of Mitochondria in Apoptosis. Annual Review of Genetics:43; 95-118.

Wang, L. 2017. Early diagnosis of breast cancer. Sensors:17(7). 
Wei MC, Lindsten T, Mootha VK, Weiler S, Gross A, Ashiya M, Thompson CB, Korsmeyer SJ. 2000. tBID, a membrane-targeted death ligand, oligomerizes BAK to release cytochrome $c$. Genes:14;2060-2071.

White, E. 2007. Role of Metabolic Stress Responses of Apoptosis and Autophagy in Tumour Suppression. Ernst Schering Foundation Symposium Proceedings:4;23-34.

Xu, W, Yang, Z, Zhou, S \& Lu, N. 2014. Posttranslational regulation of phosphatase and tensin homolog (PTeN) and its functional impact on cancer behaviours. Drug Design, Development and Therapy:8;1745-1751.

Yang, Q, She, H, Gearing, M, Colla, E, Lee, M, Shaka, JJ \& Mao, Z. 2009. Regulation of Neuronal Survival Factor MEF2D by Chaperone Mediated Autophagy. Science:323(5910);124-127.

Yang, Z \& Klionsky, DJ. 2010. Mammalian autophagy: core molecular machinery and signalling regulation. Current opinion in Cell Biology:22(2);124-131.

Yang, M, Lin, P, Hsiao, H \& Lin, S. 2012. Induction of Cellular Senescence by Doxorubicin Is Associated with Upregulated miR-375 and Induction of Autophagy in K562 Cells. PLoS ONE:7(5).

Yang, F, Teves, SS, Kemp, CJ \& Henikoff, S. 2014. Doxorubicin, DNA torsion, and chromatin dynamics. Biochimica et Biophysica Acta:1845(1);84-89.

Yang, Teves, K. and H. 2015. Doxorubicin, DNA torsion, and chromatin dynamics. Biochimica et Biophysica Acta:1845(1);84-89.

Yoon, S \& Seger, R. 2006. The extracellular signal-regulated kinase: Multiple substrates regulate diverse cellular functions. Growth Factors:24(1);21-44.

You, L, Jin, S, Zhu, L \& Qian, W. 2017. Autophagy, autophagy-associated adaptive immune responses and its role in hematologic malignancies. Oncotarget:8(7);12374-12388.

Young, AR, Narita, M, Ferreira, M, Kirschner, K, Sadaie, M, Tavare, S, Watt, FM \& Narita, M. 2009. Autophagy mediates the mitotic senescence transition. Genes and Development:23;798-803.

Zha, J, Harada, H, Yang, E \& Korsmeyer, JS. 1996. Serine Phosphorylation of Death Agonist BAD in Response to Survival Factor Results in Binding to 14-3-3 Not BCL-XL. Cell:87;619628.

Zhang, H. 2007. Molecular Signalling and Genetic Pathways of Senescence: Its Role in Tumorigenesis and Aging. Journal of Cellular Physiology:210;567-574. 


\section{Appendices}

\section{Appendix A}

Senescence associated Beta-galactosidase staining solution

Before use, all kit components must be thawed and mixed thoroughly until homogenous.

\begin{tabular}{|c|c|}
\hline Component & Stock Solution \\
\hline $\begin{array}{l}\text { Fixation buffer } \\
(20 \% \quad \text { Formaldehyde, } \\
\text { Gluteraldehyde, } \quad 2 \% \\
\mathrm{Na}_{2} \mathrm{HPO}_{4}, 14.7 \mathrm{mM} \mathrm{KH}_{2} \mathrm{PO}_{4}, 1.37 \\
\mathrm{M} \mathrm{NaCl} \text { and } 26.8 \mathrm{mM} \mathrm{KCl}^{2}\end{array}$ & $10 x$ \\
\hline $\begin{array}{l}\text { Reagent B } \\
\text { Potassium Ferricyanide }\end{array}$ & $400 \mathrm{mM}$ \\
\hline $\begin{array}{l}\text { Reagent C } \\
\text { Potassium Ferrocyanide }\end{array}$ & $400 \mathrm{mM}$ \\
\hline $\begin{array}{l}\text { X-gal solution } \\
\text { chloro-3-indolyl- } \beta-\end{array}$ & $40 \mathrm{mg} / \mathrm{ml}$ \\
\hline Staining Solution & $10 x$ \\
\hline $\begin{array}{l}\text { Phosphate buffered saline } \\
\text { (PBS) }\end{array}$ & $10 x$ \\
\hline Distilled H2O & \\
\hline
\end{tabular}

\section{Appendix B}

\section{PBS: 0.1 M (2 litre)}

- $16 \mathrm{~g} \mathrm{NaCl}$

- $0.4 \mathrm{~g} \mathrm{KCl}$

- $2.88 \mathrm{~g} \mathrm{Na}_{2} \mathrm{HPO}_{4}$

- $0.48 \mathrm{~g} \mathrm{KH}_{2} \mathrm{PO}_{4}$

All reagents are dissolved in 1.8 litres of $\mathrm{dH}_{2} \mathrm{O}(\mathrm{pH} 7.4)$ and the final volume is adjusted to 2 litres. 


\section{Appendix C}

\section{Modified RIPA buffer (100ml)}

Add to $50 \mathrm{ml}$ of $\mathrm{dH}_{2} \mathrm{O}$ :

- 790mg Tris-base

- $900 \mathrm{mg} \mathrm{NaCl}$

Stir until dissolved, adjust $\mathrm{pH}$ to 7.4

- Add $10 \mathrm{ml}$ of $10 \%$ NP-40 [1\%]

- $10 \mathrm{ml}$ of $10 \%$ Na-deoxycholate $\left(1 \mathrm{~g}\right.$ in $\left.10 \mathrm{ml} \mathrm{dH}_{2} \mathrm{O}\right)$

Stir solution until it turns clear.

- Add $5 \mathrm{ml}$ of $100 \mathrm{mM}$ EDTA ( $5 \mathrm{mM})$ (1.46g EDTA in $\left.50 \mathrm{ml} \mathrm{dH}_{2} \mathrm{O}, \mathrm{pH} 8.0\right)$

- Add $5 \mathrm{ml}$ of $100 \mathrm{mM}$ EGTA ( $5 \mathrm{mM})$ (1.90g EGTA in $50 \mathrm{ml} \mathrm{dH}_{2} 0$, pH 8.0)

- $1 \mathrm{ml}$ of $10 \%$ SDS

Adjust to $100 \mathrm{ml}$ with $\mathrm{dH}_{2} \mathrm{O}$, aliquot into tubes and store in a $4^{\circ} \mathrm{C}$ fridge.

\section{Appendix D}

\section{Bradford Assay:}

1. If needed, thaw protein samples on ice

2. Thaw a $1 \mathrm{mg} / \mathrm{ml}$ BSA stock solution

3. Prepare BSA working solution $(200 \mu \mathrm{g} / \mathrm{ml})$ by diluting $100 \mu \mathrm{l} \mathrm{BSA}$ with $400 \mu \mathrm{dH}_{2} \mathrm{O}(50 \mathrm{ul}$ $\mathrm{BSA}+450 \mathrm{ul} \mathrm{dH}_{2} \mathrm{O}$ if using $2 \mathrm{mg} / \mathrm{ml}$ BSA stock). Vortex

4. Prepare Bradford standards as follow (in duplicate):

\begin{tabular}{|l|l|l|l|}
\hline$\mu \mathrm{g}$ Protein & $200 \mu \mathrm{g} / \mathrm{ml} \mathrm{BSA}$ & $\mathrm{dH}_{2} \mathrm{O}$ & Bradford Reagent \\
\hline 0 (Blank) & $0 \mu \mathrm{l}$ & $100 \mu \mathrm{l}$ & $900 \mu \mathrm{l}$ \\
\hline 2 & $10 \mu \mathrm{l}$ & $90 \mu \mathrm{l}$ & $900 \mu \mathrm{l}$ \\
\hline 4 & $20 \mu \mathrm{l}$ & $80 \mu \mathrm{l}$ & $900 \mu \mathrm{l}$ \\
\hline 8 & $40 \mu \mathrm{l}$ & $60 \mu \mathrm{l}$ & $900 \mu \mathrm{l}$ \\
\hline 12 & $60 \mu \mathrm{l}$ & $40 \mu \mathrm{l}$ & $900 \mu \mathrm{l}$ \\
\hline 16 & $80 \mu \mathrm{l}$ & $20 \mu \mathrm{l}$ & $900 \mu \mathrm{l}$ \\
\hline 20 & $100 \mu \mathrm{l}$ & $0 \mu \mathrm{l}$ & $900 \mu \mathrm{l}$ \\
\hline
\end{tabular}


5. Vortex protein samples

6. For each Bradford sample, add $5 \mu$ l of protein sample to $95 \mu \mathrm{ld} \mathrm{H}_{2} \mathrm{O}$ and $900 \mu \mathrm{l}$ Bradford reagent. Prepare samples in duplicate.

7. Vortex Bradford samples and standards

8. Zero spectrophotometer with blank and set @ 595nm

9. Read absorbencies

10. Draw standard curve in Excel graph and plot values of sample to determine [protein sample]

\section{Sample Preparation (for western blot):}

1. Prepare a working solution of Laemmli's sample buffer

- $\quad 850 \mu l$ Laemmli's stock buffer solution $+150 \mu l \beta$-mercaptoethanol

- Work in fume hood and Vortex thoroughly

2. Prepare samples by adding the appropriate amount of protein sample to sample buffer (as determined with Bradford Assay)

- In order to give equal amounts of protein for loading $=50 \mu \mathrm{g}$

- Work in fume hood

- Vortex

3. Store as ready-made samples

OR

Proceed with western blot

4. Punch a hole in the lid of each sample and boil for $5 \mathrm{~min} @ 95^{\circ} \mathrm{C}$

5. Mix by brief centrifugation (pulse)

6. Samples now ready for loading 


\section{Appendix E \\ Laemmli's Sample Buffer (3x)}

- Dissolve $9.09 \mathrm{~g}$ Tris in $100 \mathrm{ml} \mathrm{dH}_{2} \mathrm{O}$

- Add $6 \mathrm{ml} 10 \%$ SDS and stir (pH 6.8)

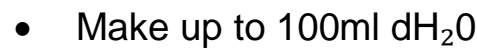

- Weigh off $60 \mathrm{~g}$ glycerol and add $99.9 \mathrm{ml}$ of above-mentioned solution

- $\quad$ Add $26.4 \mathrm{~g}$ SDS

- Add $0.225 \mathrm{~g}$ Bromophenol Blue and ensure to dissolve thoroughly

- Adjust final volume to $225 \mathrm{ml}$ with $\mathrm{dH}_{2} \mathrm{O}$

To prepare working solution, add $150 \mathrm{ul} \beta$-mercaptoethanol to $850 \mathrm{ul}$ sample buffer (in fumehood)

Final concentrations when adding sample buffer to sample in ratio of 1:2

- $\quad 62.5 \mathrm{mM}$ Tris, $\mathrm{pH} 6.8$

- $4 \%$ SDS

- $10 \%$ Glycerol

- $0.03 \%$ Bromophenol Blue

- $5 \% \beta$-mercaptoethanol 


\section{Appendix F \\ Western Blot}

\section{Day 1}

1. Prepare BioRad Fast Cast Stain-Free gels according to instructions

a. Gels can be made beforehand and stored in the fridge in wetted paper towels and sealed in cling wrap

2. Thaw protein samples on ice

a. Prepared in 2:1 ratio of protein sample + Laemli's buffer

3. Punch hole in lid of eppie, mix by vortexing briefly and boil for $5 \mathrm{~min}$ at $95^{\circ} \mathrm{C}$

4. Spin samples down with quick pulse

5. Assemble the gasket

a. Assemble the gels onto the gasket with the comb facing inside

b. Fill the gasket with running buffer - check for leaks

c. Carefully remove the combs and rinse out wells with P200 pipet (set to approx. $100 \mathrm{ul})$ and yellow tip

6. Load ladder (4 ul BLUeye)

7. Load samples

8. Place gasket into tank and fill with running buffer. Re-fill gasket if necessary

9. Attach lid (black on black, red on red) and plug into power pack

10. Run at $100 \mathrm{~V}$ for approx. $10 \mathrm{~min}$ (until sample has entered gel)

11. Increase to $120-150 \mathrm{~V}$ and run until blue dye front reaches the bottom of the gel (approx. $1 \mathrm{hr}$ )

12. When finished, transfer immediately

13. Activate stain-free properties of gel on ChemiDoc

a. Gel activation protocol, $2.5 \mathrm{~min}$

14. Prepare for transfer (few min beforehand)

a. Soak two pieces of blotting paper in transfer buffer for 2 min

b. Activate piece of PVDF membrane in $100 \%$ methanol $(10-15 \mathrm{sec})$ and then soak in transfer buffer for 2 min

c. Size $7 \mathrm{~cm} \times 8.4 \mathrm{~cm}$ - blotting paper and membrane should be same size, preferably gel also

15. Assemble transfer sandwich (on TurboBlot tray)

a. Place one piece of blotting paper

b. Place membrane on top of blotting paper. Roll to remove bubbles

c. Place gel on top of membrane 
i. If transferring two gels in one tray, orientate gels with bottom towards centre

d. Place second piece of blotting paper on top of gel. Carefully roll to remove bubbles

e. Close with lid - be careful not to move lid while closing

16. Transfer on TurboBlot according to desired settings

a. Biorad defined HMW setting generally works fine

17. After transfer, disassemble stack

a. Blotting paper can be re-used

b. Gel can be visualised to determine transfer efficiency if desired

18. Label membrane and rinse in $100 \%$ methanol

a. Labelling - only use soft pencil (black art pencil works well) and not markers or pens (causes smudges)

b. Either label membrane while still on transfer stack or place membrane only on ethanol cleaned surface (white tray from Chemidoc works well)

19. Air-dry membrane

a. Becomes white

b. Hold in fumehood for quicker drying

20. Re-hydrate membrane in $100 \%$ methanol

a. Until translucent, $10-15 \mathrm{sec}$

21. Wash membrane in TBS-T for $5 \mathrm{~min}$

22. Image transfer on membrane on Chemidoc

a. Stain-free blot setting

b. If using for normalisation, make sure image is clean and not over-exposed

23. Block membrane in $5 \%$ milk (prepared in TBS-T) for $1 \mathrm{hr}$ with gentle shaking

a. Or 5\% BSA (prepared in TBS-T) for when probing for phosphorylated proteins

24. Wash membrane $3 x$ for 5 min in TBS-T

25. Incubate membrane on primary antibody $\mathrm{O} / \mathrm{N}$ at $4^{\circ} \mathrm{C}$

a. Prepared in $50 \mathrm{ml}$ centrifuge tube

b. Dilute antibody in TBS-T to desired concentration, normally 1:1000

c. Attach to rotators in walk-in fridge

i. Make sure tube/s are straight and rotor is balanced 


\section{Day 2}

1. Retrieve membranes

a. Store primary antibody in fridge/freezer

b. Mark on tube how many times primary antibody has been used

2. Wash membrane $3 \times 5$ min in TBS-T

3. Incubate membrane on secondary antibody for $1 \mathrm{hr}$ at RT

a. Prepared in $50 \mathrm{ml}$ centrifuge tube

b. Dilute antibody in TBS-T to desired concentration, normally 1:10 000

c. Place on roller in WB room

4. Wash membrane $3 \times 5 \mathrm{~min}$ in TBS-T

5. Develop
a. Prepare minimum amount of ECL needed in Eppendorf tube in 1:1 ratio
b. Place membrane on Chemidoc and check position
c. Add ECL to the desired area and roll to ensure even spread. Roll away excess ECL
d. Expose using desired settings 


\section{Appendix G \\ Immunocytochemistry}

1. Seed cells onto sterile glass coverslips, in 6-well plates, and perform experiments as usual

2. Remove coverslips from wells and place onto dry slides. By keeping the slide dry, it will ensure that the liquid stays only on the coverslip

3. Gently wash twice with PBS (drop onto coverslip until covered)

4. Fix cells for 3-5 min at RT

5. Wash three times with PBS

6. Block for $30-60 \mathrm{~min}$ at RT - use $200 \mu$ l per coverslip

7. Remove blocking solution (do not wash)

8. Add primary antibody and incubate $\mathrm{O} / \mathrm{N}$ at $4^{\circ} \mathrm{C}$ - use 80-100 $\mu$ l per coverslip. Can be prepared in blocking solution or in PBS. Secondary antibody controls should be incubated with PBS

9. Wash three times with PBS

10. Add secondary antibody and incubate for $1 \mathrm{hr}$ at RT, protected from light - use 80-100 $\mu \mathrm{l}$ per coverslip. Can be prepared in blocking solution or in PBS.

11. Wash three times with PBS

12. Counterstain nuclei with Hoechst $(10 \mu \mathrm{g} / \mathrm{ml}$, prepared in PBS) for $10 \mathrm{~min}$

13. Wash three times with PBS

14. Carefully push the coverslip off slide and onto a paper towel (remember to keep it wet)

15. Clean and dry the slide as needed and place a small drop of mounting media onto the slide

16. Drain off excess PBS from the coverslip by holding a corner against a paper towel. Gently drop the coverslip onto the drop of mounting media with the cells facing downward to the slide

17. Leave to dry for $30-60$ min at RT

18. Seal the slide with clear nail polish and leave to dry for another 30-60 min at RT

19. Store at $-20^{\circ} \mathrm{C}$, protected from light, until ready to image. 


\section{Appendix $\mathrm{H}$ \\ Image Processing}

1. Deconvolute image stack (Huygens Professional Software/ Olympus Cell^R Software).
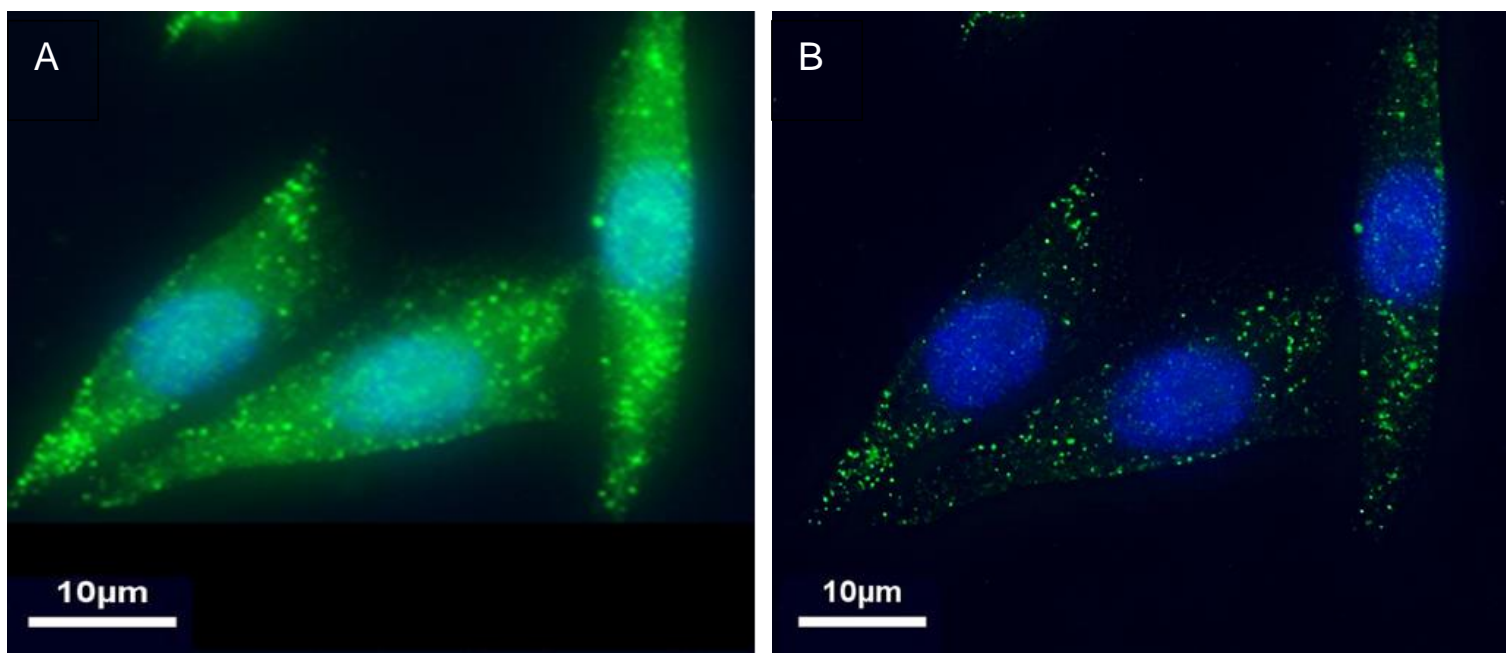

Figure 1. Image deconvolution. A) Unprocessed raw z-stack (maximum intensity projection for sake of illustration). B) Deconvoluted z-stack (maximum intensity projection shown for sake of illustration).

2. Make a maximum intensity projection of the deconvoluted z-stack (ImageJ).

3. Split channels into respective signal subsets (ImageJ).

4. Threshold images using adaptive Otsu thresholding (CellProfiler 3.1.5).
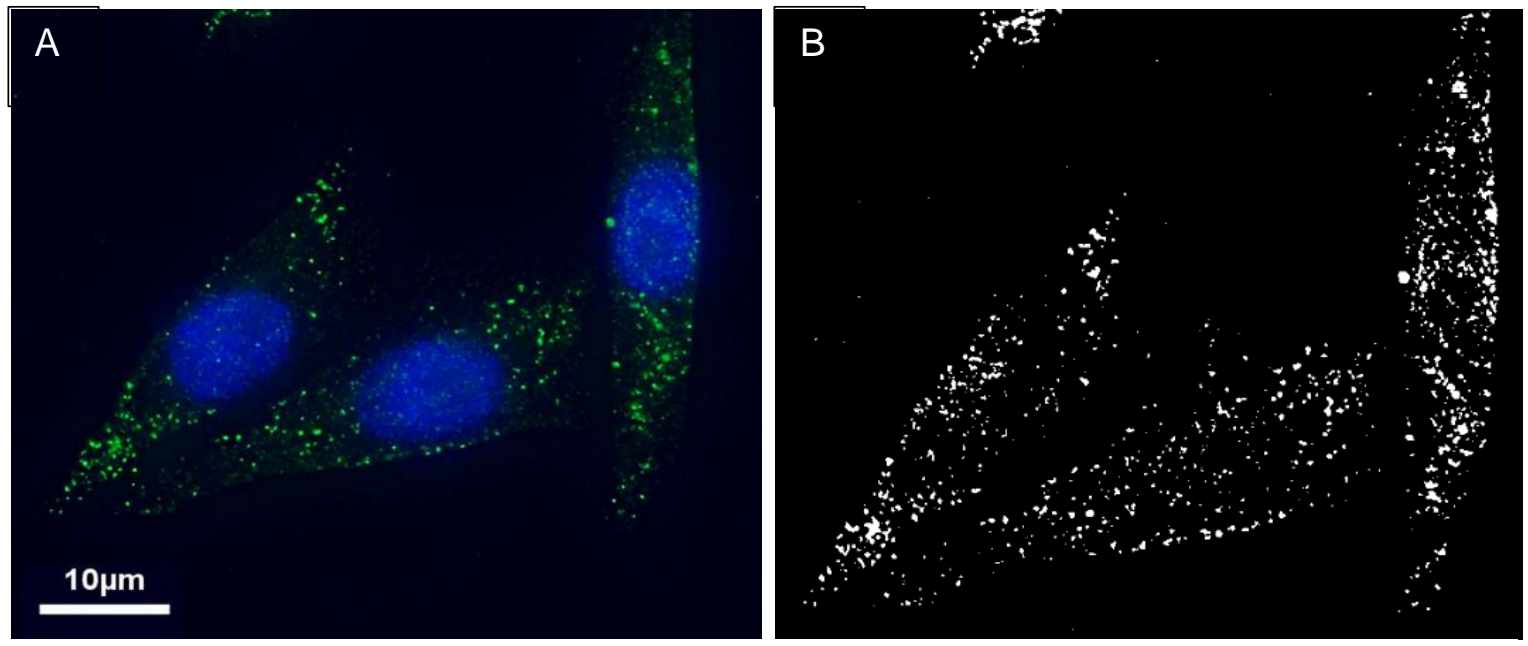

Figure 2. Image thresholding. A) Unthresholded image containing both Hoescht and GFP signal. B)

Thresholded image of GFP signal only. 
5. Draw region of interest $(\mathrm{ROI})$ around cells and save each $\mathrm{ROI}$ using $\mathrm{ROI}$ manager in ImageJ. Proceed with Particle Count (ImageJ). The total amount of particles will be displayed.
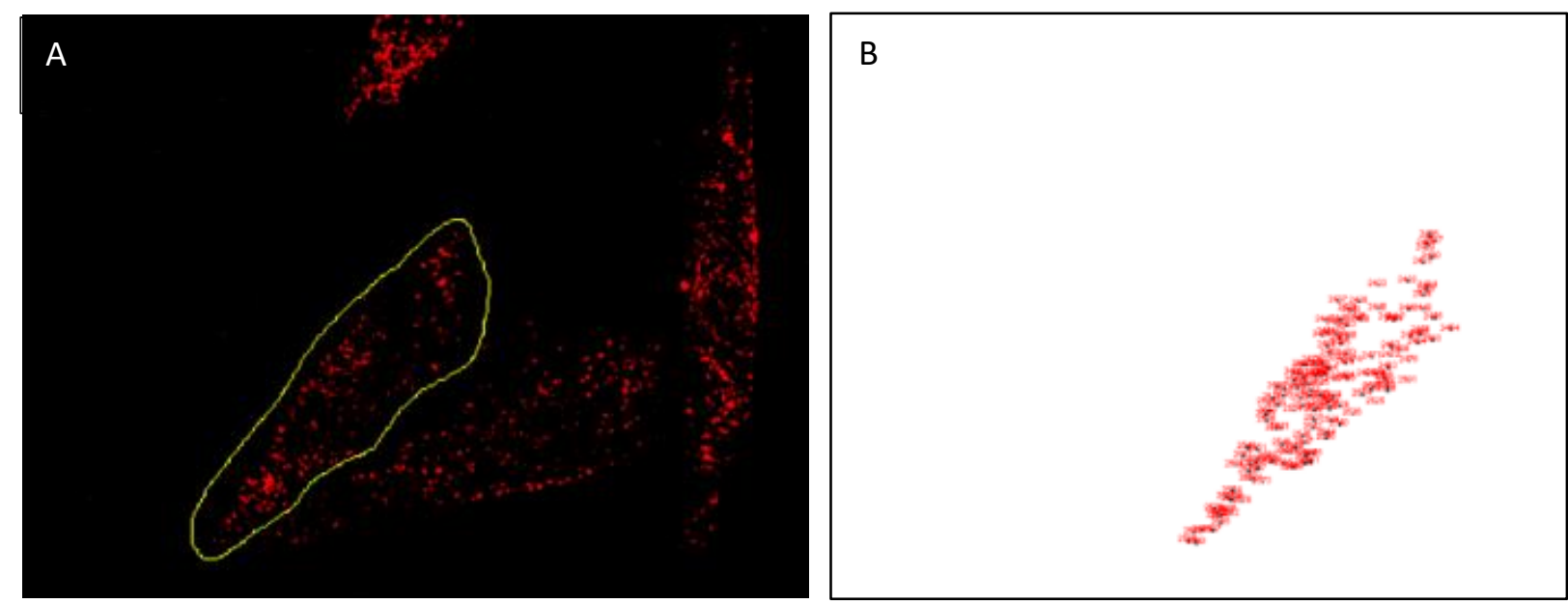

Figure 3. Particle counts of ROls. A) Region of interest drawn around cell. B) Particle count of ROI. 